\title{
Single-molecule tracking tools to study plasma membrane receptor dynamics
}

Applications to EGF receptor

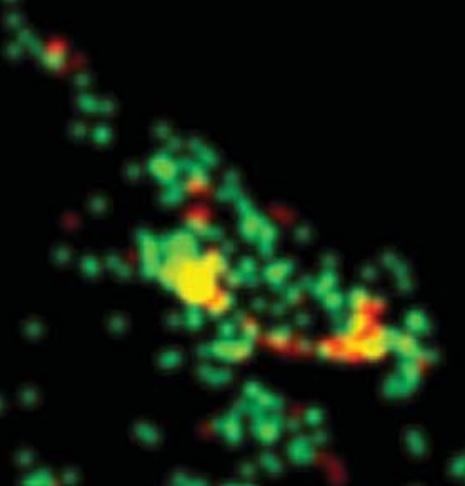

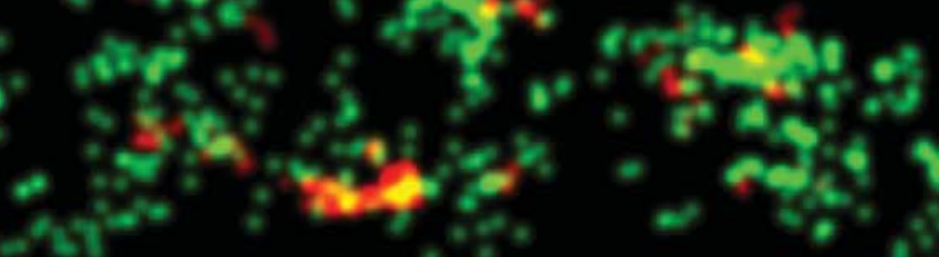

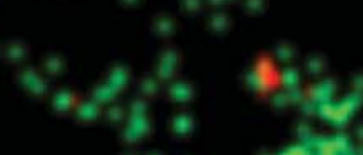

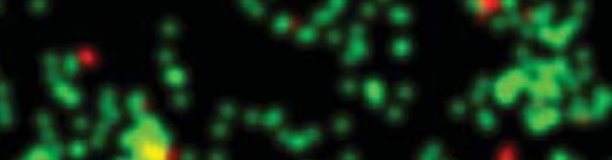

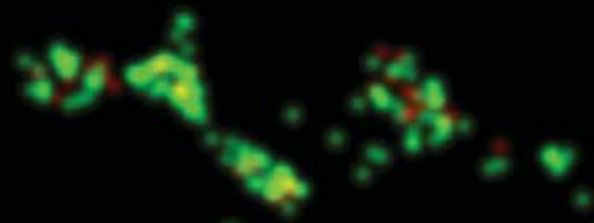

(18:8,

4. 1.

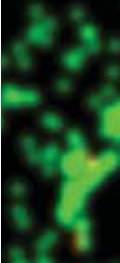

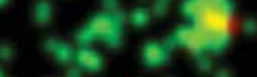

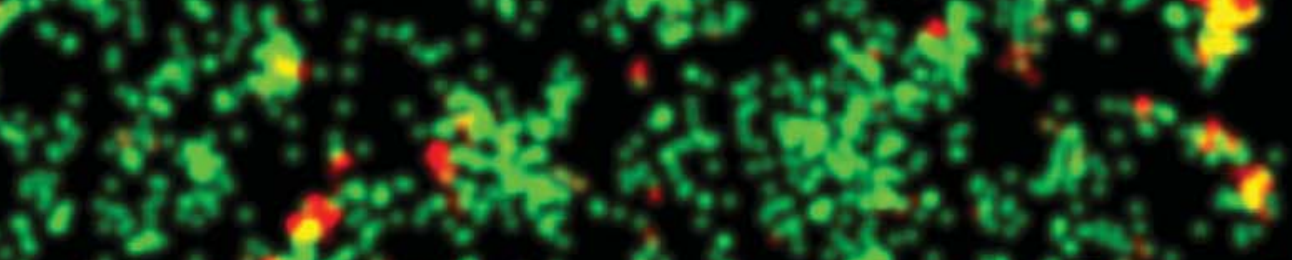

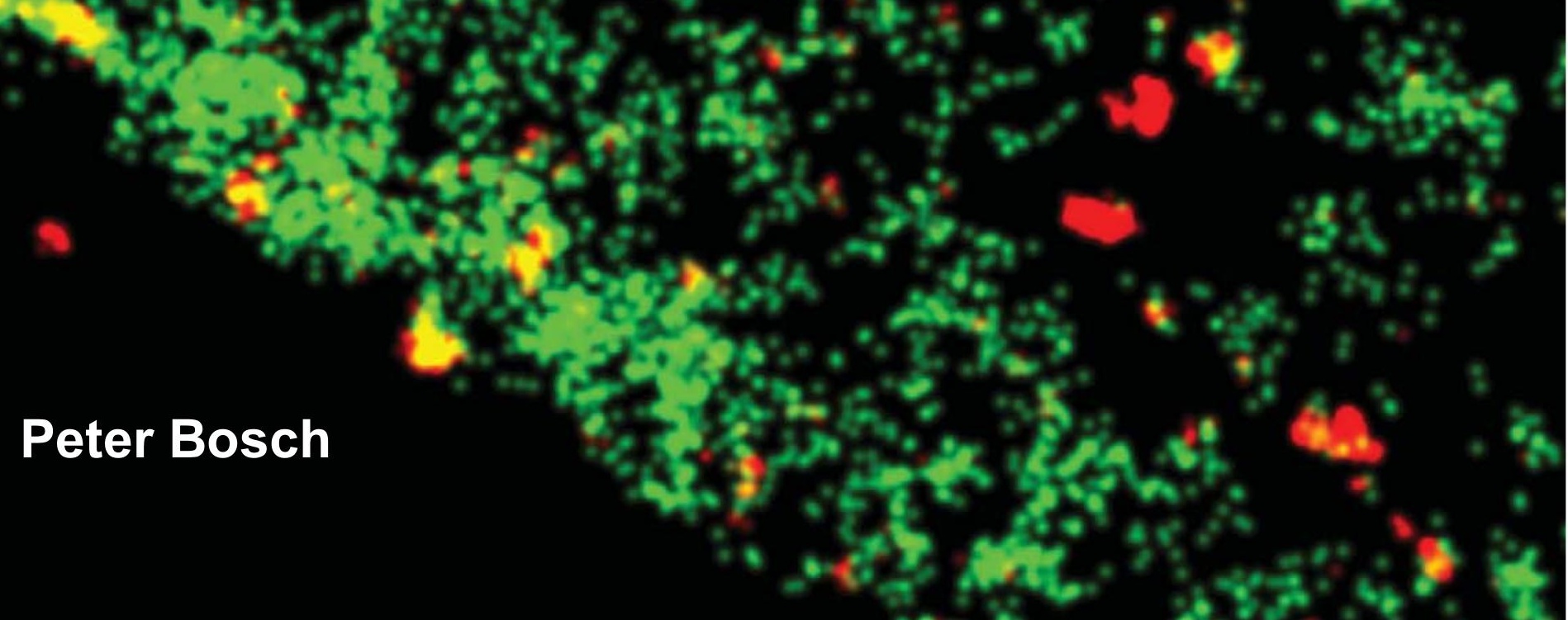




\section{SINGLE-MOLECULE TRACKING TOOLS TO STUDY PLASMA MEMBRANE RECEPTOR DYNAMICS}

APPLICATIONS TO EGF RECEPTOR

Peter Johannes Bosch 


\section{Members of the dissertation committee}

Prof. dr. V. Subramaniam

Dr. ir. J.S. Kanger

Prof. dr. ir. J.W.M. Hilgenkamp

Prof. dr. W.J. Briels

Prof. dr. ir. L. Brunsveld

Prof. dr. J.L. Herek

Prof. dr. K.A. Lidke

Prof. dr. T. Schmidt
University of Twente (supervisor)

University of Twente (assistant supervisor)

University of Twente (chairman and secretary)

University of Twente

Eindhoven University of Technology

University of Twente

University of New Mexico, USA

Leiden University

\section{Cover}

The spatial distribution of the diffusion states exhibited by EGF receptors in an MCF7 cell. The reconstructed image shows the areas where receptors were classified in the fast diffusion state as green, and areas where receptors were classified in the slow diffusion state as red.

Cover design by M.C. Bosch

The work described in this thesis was performed at the Nanobiophysics group, MESA+ Institute for Nanotechnology, Faculty of Science and Technology, University of Twente, PO Box 217, 7500 AE, Enschede, The Netherlands.

This research has been financially supported by NanoSciE+ through STW grant 11022:

NanoActuate.

Peter Johannes Bosch

Single-molecule tracking tools to study plasma membrane receptor dynamics: Applications to EGF receptor

Ph.D. Thesis, University of Twente, The Netherlands

ISBN: 978-90-365-3694-3

DOI: $10.3990 / 1.9789036536943$

Copyright @ 2014, P.J. Bosch

All rights reserved.

\section{(우요 $\odot$}

This work is licensed under the Creative Commons Attribution-NonCommercial-NoDerivatives 4.0 International Public License. To view a copy of this license, visit http://creativecommons.org/licenses/by-nc-nd/4.0/ or send a letter to Creative Commons, 171 Second Street, Suite 300, San Francisco, California 94105, USA. 


\title{
SINGLE-MOLECULE TRACKING TOOLS TO STUDY PLASMA MEMBRANE RECEPTOR DYNAMICS
}

\author{
APPLICATIONS TO EGF RECEPTOR
}

DISSERTATION

to obtain

the degree of doctor at the University of Twente, on the authority of the rector magnificus, prof. dr. H. Brinksma, on account of the decision of the graduation committee, to be publicly defended on Thursday the 3rd of July 2014 at 12.45 hours

by

Peter Johannes Bosch

born on the 13th of July, 1984

in Leeuwarden, The Netherlands 
This dissertation has been approved by:

Prof. dr. V. Subramaniam

Promotor

Dr. ir. J.S. Kanger

Assistant promotor 


\section{Declaration}

I hereby declare that this submission is my own work and that, to the best of my knowledge and belief, it contains no material previously published or written by another person nor material which to a substantial extent has been accepted for the award of any other degree or diploma of the university or other institute of higher learning, except where due acknowledgment has been made in the text.

All statements are based on a comprehensive review of the literature to the date of the experiment. Experiments for this thesis were performed in the period $2010-2013$.

\section{Style}

This thesis is written in a combination of British and American English. The use of American English is required for submission of some of our chapters to American journals. We have tried to maintain a particular choice per chapter.

\section{Abstract}

In this thesis we developed tools to utilize single-molecule tracking microscopy to study the signalling mechanism of the ErbB family of receptors, and in particular of the epidermal growth factor receptor (EGFR). This receptor family is prototypical of receptor tyrosine kinases (RTK) and is implicated in the development and progression of various human cancers, and therefore form attractive targets for drug discovery. We related the diffusion speed of EGFR to its dimerization state, which is an essential step in its central role of transducing extracellular signals into cellular outcomes. Malignancies therein and drug-induced dimerizations are therefore important research topics for therapeutic application. Our single-molecule tracking data of EGFR and ErbB3 revealed particular molecular interactions in tumour cells, and detected altered behaviour when drugs are applied which are especially designed to prevent such molecular interactions. Before we were able to record trajectories of EGFR, and analyse these in term of dimerization and interactions with other cellular structures upon addition of ligand and antagonists (chapter 5), we realized a microscope for this purpose (chapter 2), devised a framework to analyse trajectories in term of different diffusion populations (chapter 3), and further advanced an existing protein labelling system - SNAP-tag - to the single molecule level (chapter 4). The research described in this thesis offers desirable advancements to this proteintag labelling system for application in single-molecule imaging and tracking, and to the postmeasurement analysis of protein trajectories recorded by introducing a classification framework for multiple populations of diffusion, to be able to investigate dynamic protein motion and interactions in live cells. We concluded our thesis with a proof of principle that locally induced stimulation of receptor by ligand functionalized AFM tips can provide additional insight in molecular interactions when combined with single-molecule tracking (chapter 6). 


\section{Table of contents}

1. Introduction . .9

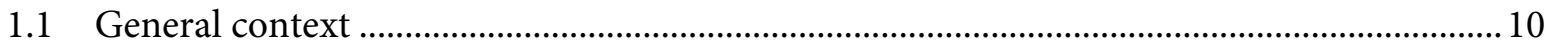

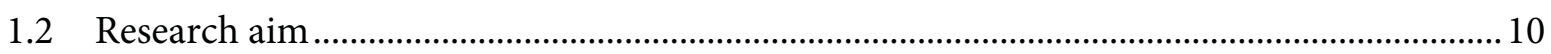

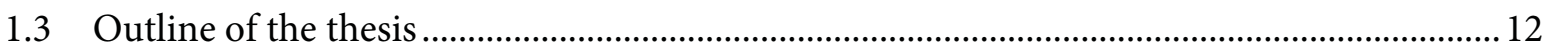

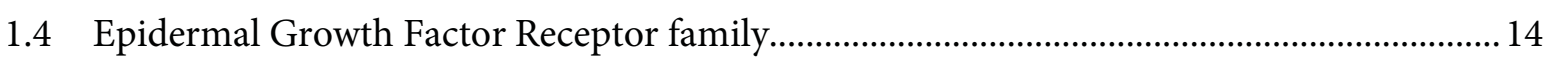

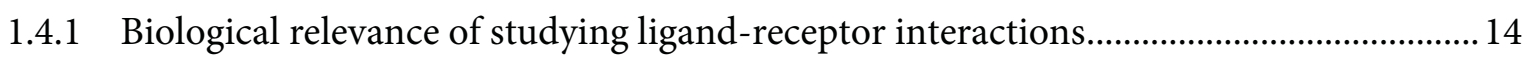

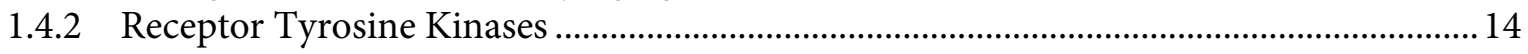

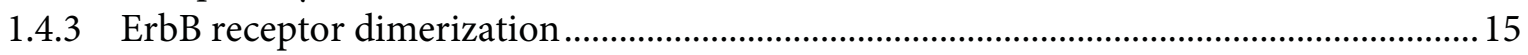

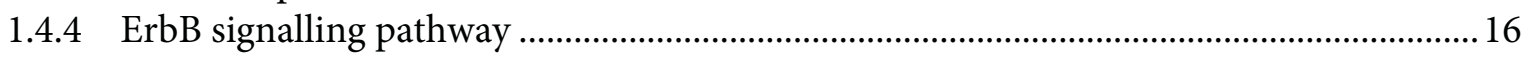

1.4.5 Membrane domains and the involvement of ErbB receptors ........................................... 17

1.5 Single-molecule Tracking Microscopy ……............................................................................. 18

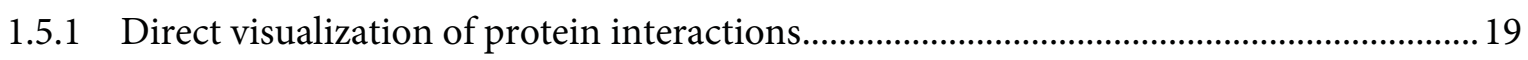

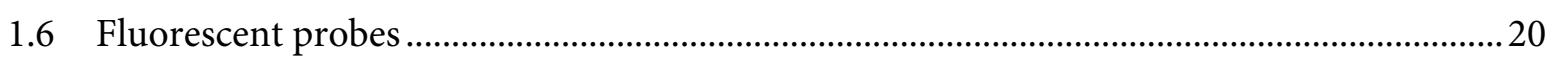

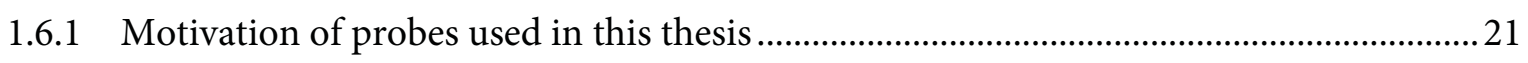

1.7 Controlled receptor activating techniques...........................................................................22

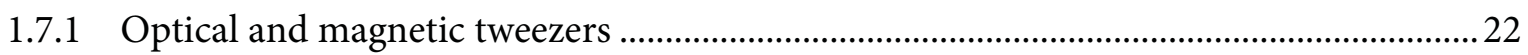

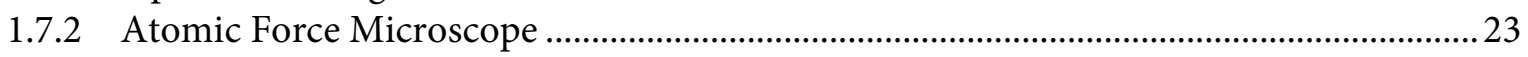

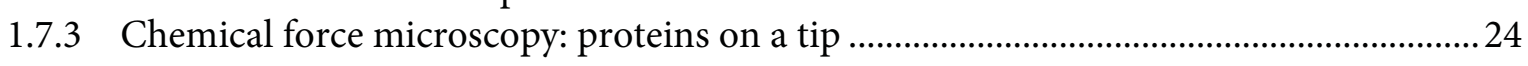

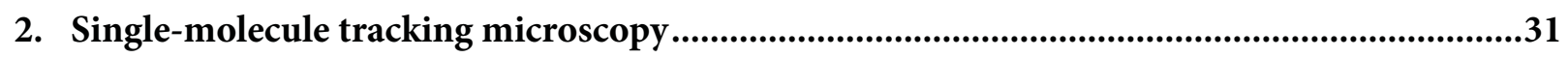

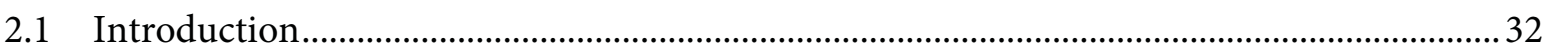

2.2 Design and realization of a single-molecule tracking microscope .......................................... 32

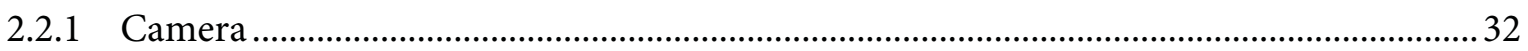

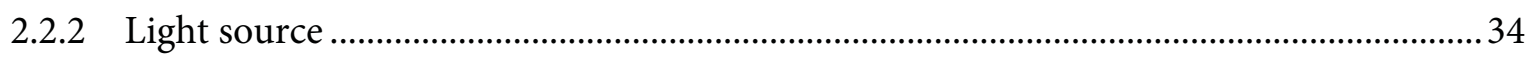

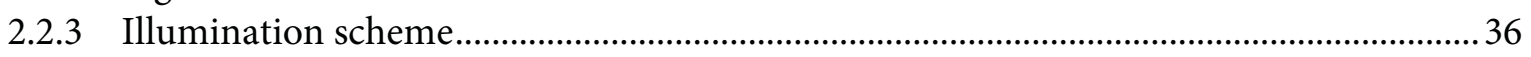

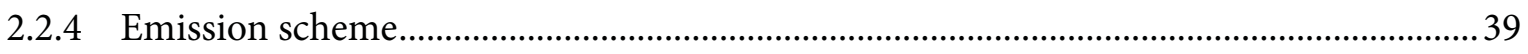

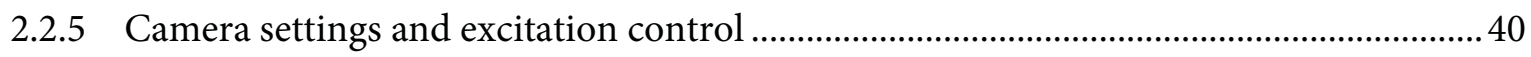

2.3 Results: characterization and performance of the setup .......................................................42

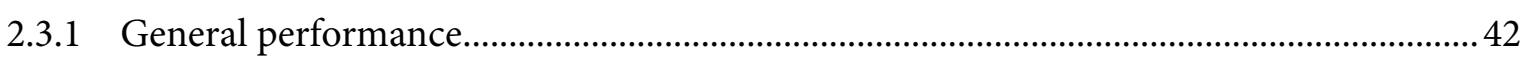

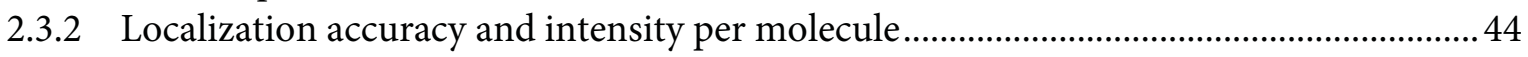

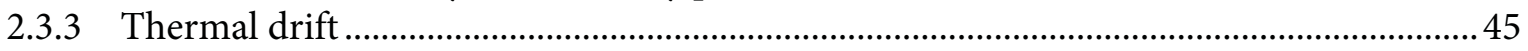

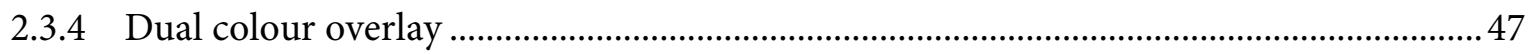

2.4 Tracking single molecules: from recordings to trajectories....................................................49

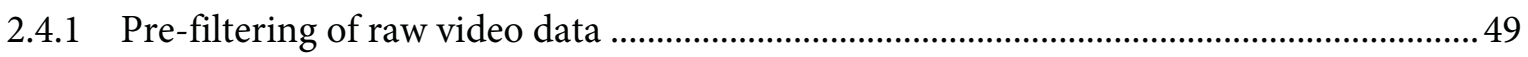

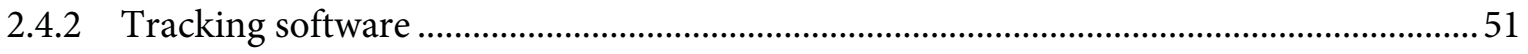

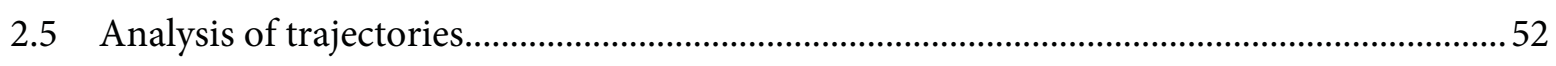

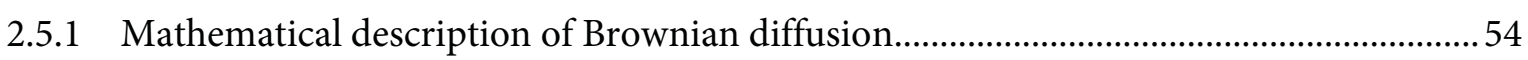

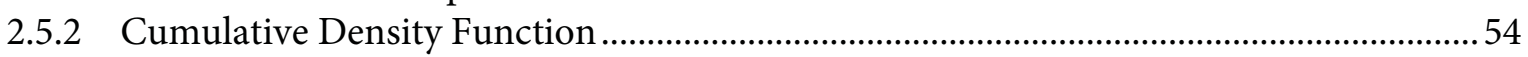

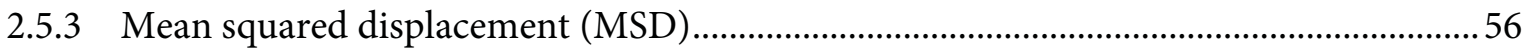

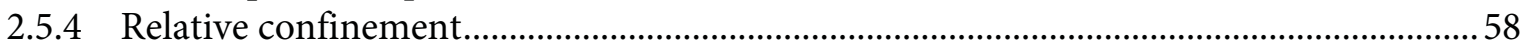




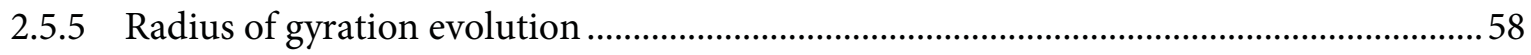

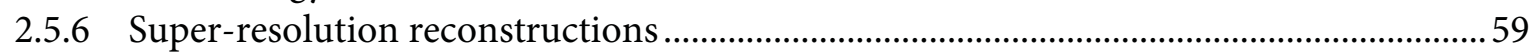

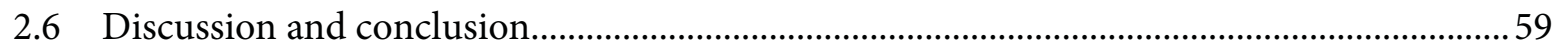

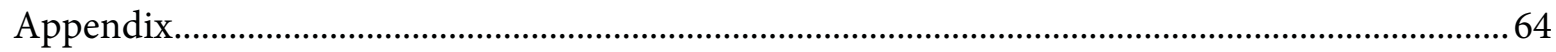

3. Classification of dynamical diffusion states in single molecule tracking microscopy.............67

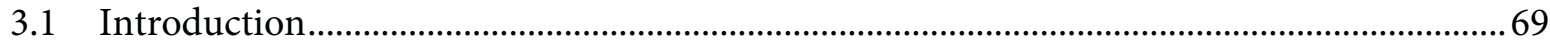

3.1.1 Proposed scheme for diffusion state classification ....................................................... 70

3.1.2 Existing motion classification schemes............................................................................. 71

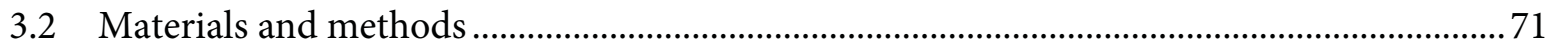

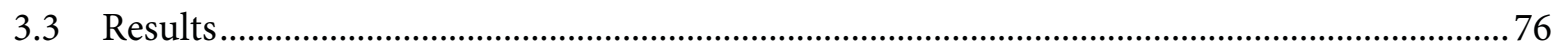

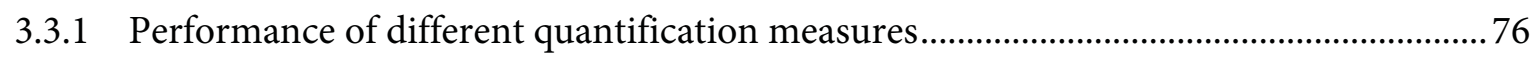

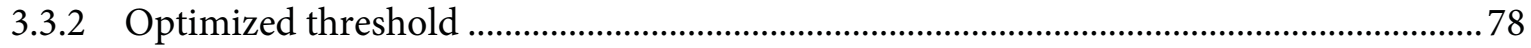

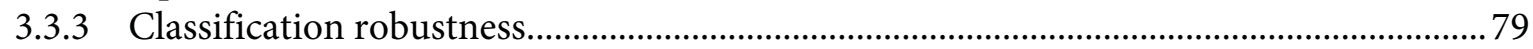

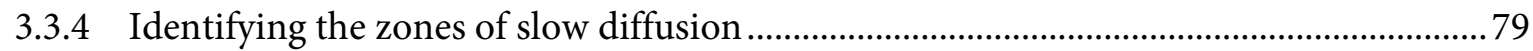

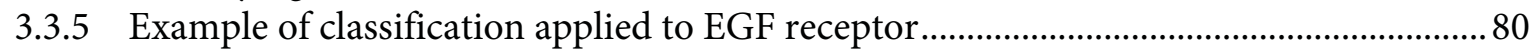

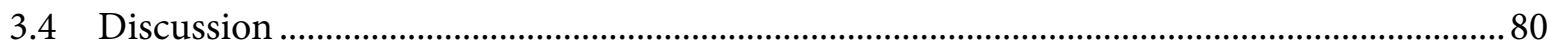

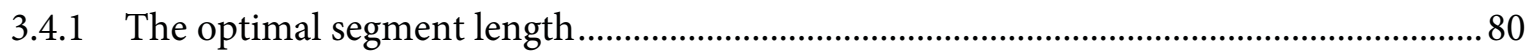

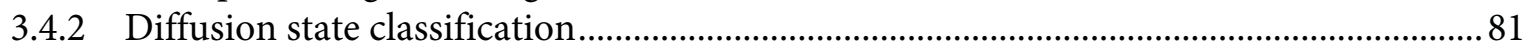

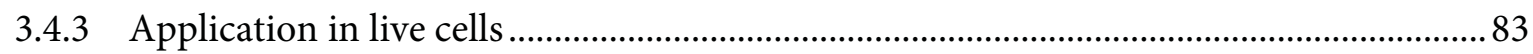

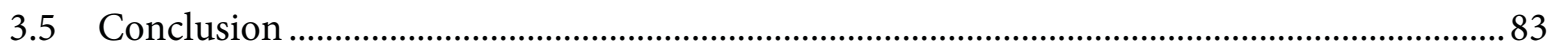

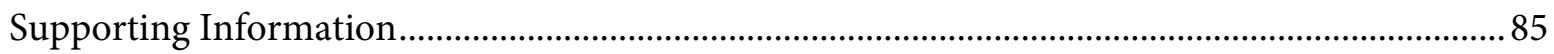

4. Evaluation of fluorophores to label SNAP-tag fused proteins for multicolor single molecule tracking microscopy in live cells....................................................................................99

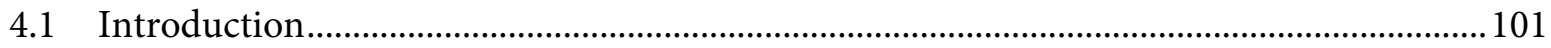

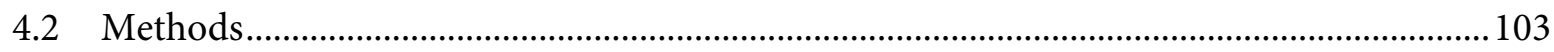

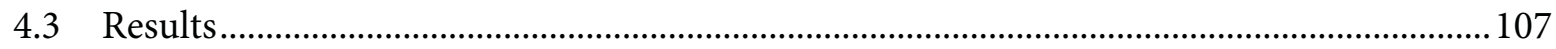

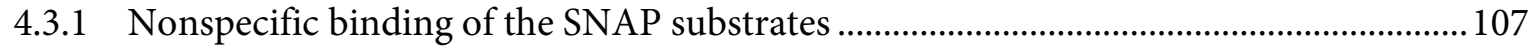

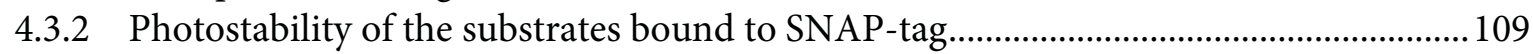

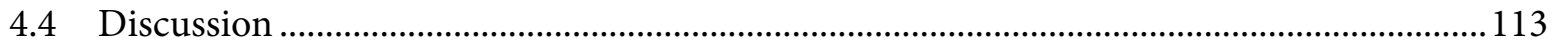

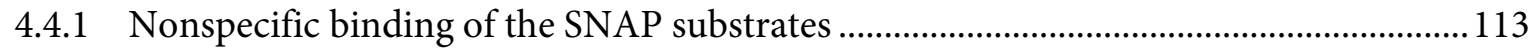

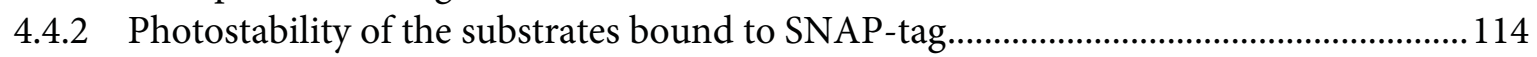

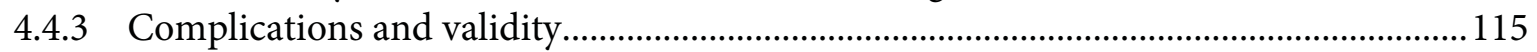

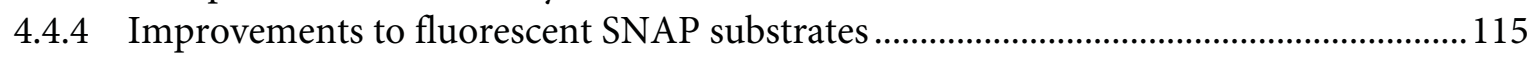

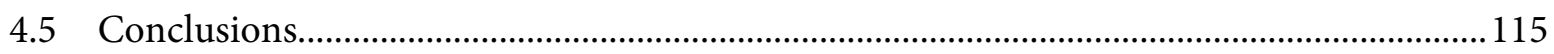

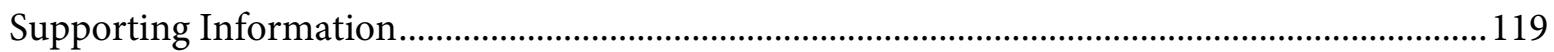

5. The diffusion of single EGF receptors relates to their dimerization state and is altered by antagonists.

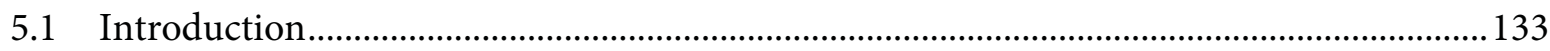

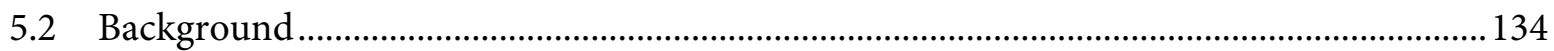

5.2.1 Molecular interactions of ErbB receptors upon kinase inhibition and cetuximab ........ 134

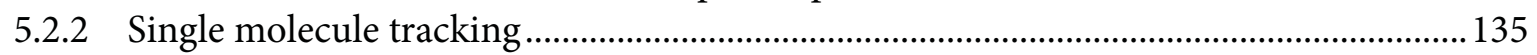

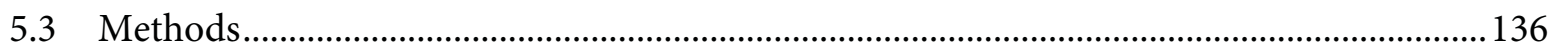




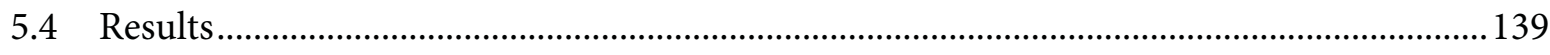

5.4.1 EGF induced dimerization and pre-clustering are reflected in the diffusion of

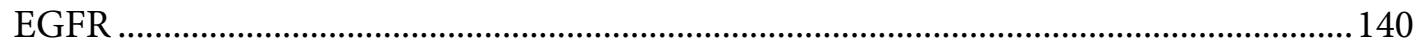

5.4.2 The slow population of EGF receptor is transient and is not influenced by ligand

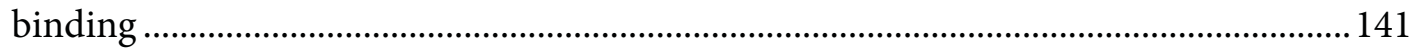

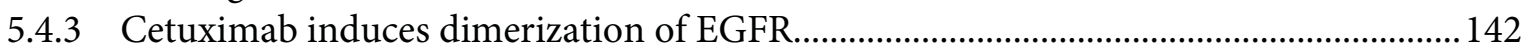

5.4.4 An active kinase domain is essential for stable dimerization of EGFR............................ 142

5.4.5 ErbB3 is removed from preformed clusters upon cetuximab treatment but not

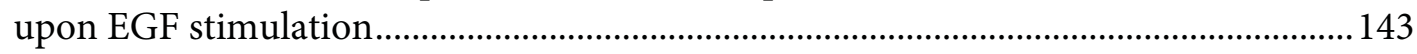

5.4.6 The spatial organization of regions where receptors transiently experience strong membrane interactions are independent of the receptor dimerization state................... 144

5.4.7 Direct dimerization observations using single molecule tracking conflicts with

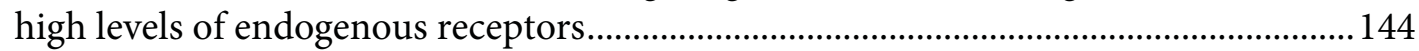

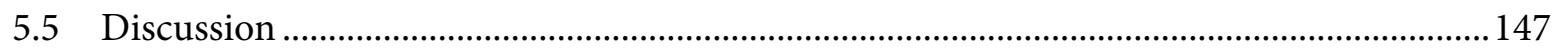

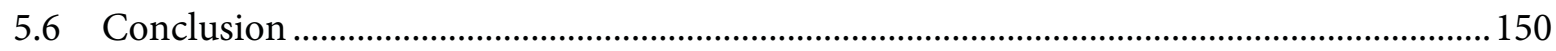

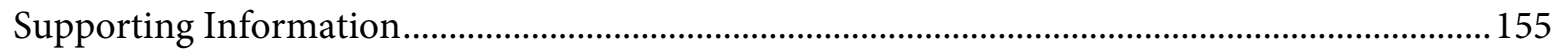

6. Single-molecule tracking of plasma membrane proteins combined with local actuation using ligand functionalised AFM tips................................................................................................163

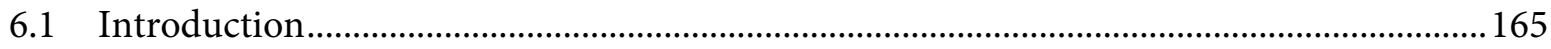

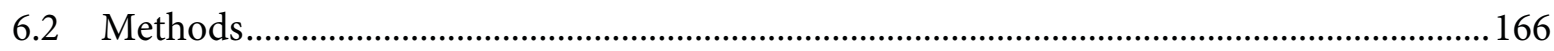

6.3 Testing the functionalization of AFM tips with force spectroscopy ……........................... 170

6.4 Testing the functionalization of AFM tips by an immunofluorescence assay .....................171

6.5 Selection of an AFM tip for use in single-molecule optical microscopy............................... 173

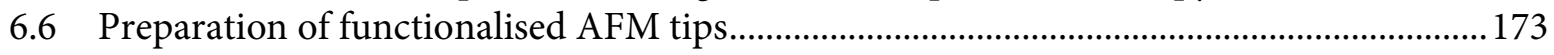

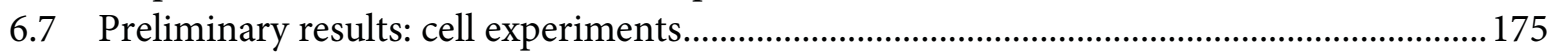

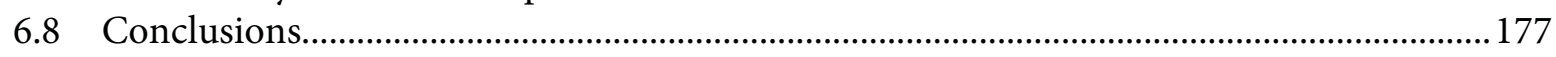

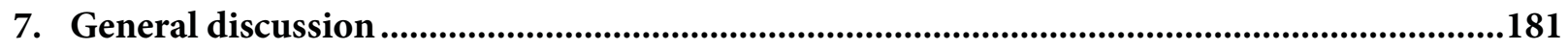

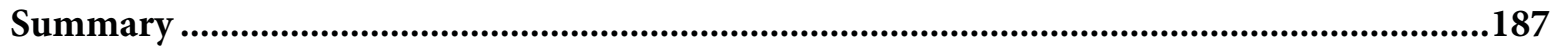

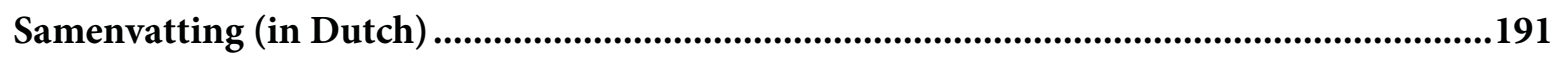

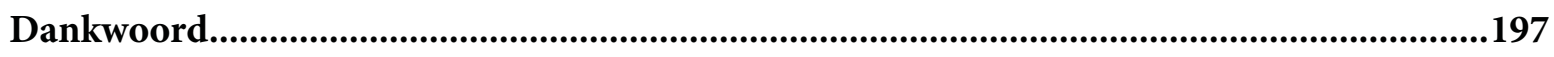

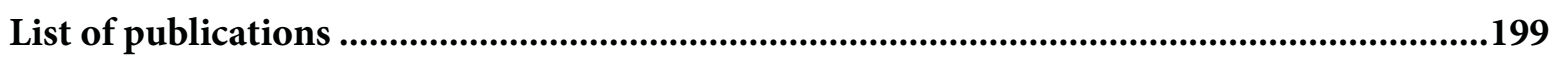

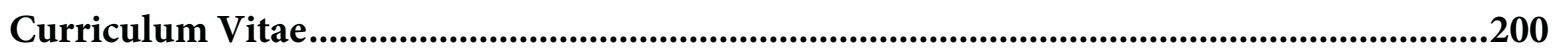




\section{Chapter 1}

Introduction 


\subsection{General context}

Life is composed of the incredible interplay of cells. With numerous processes occurring within a few micrometres, cells make up the existence of a bewildering range of organisms. Yet while we now know the complete sequence of the genome coding for proteins, the molecular interplay of interactions of proteins with their substrates are far from understood. Certainly, understanding protein interactions inside and between cells is of great importance for progress in understanding the fundamentals behind the complex cellular machinery that makes up life. The reason why studying these processes in the physiological context is so difficult is because microscopy techniques able to image or probe these protein interactions within living cells at real time are still limited. Although more techniques are available to study proteins in model systems, these systems require extraction of the protein from its native environment, resulting in loss of information about its role within the ultrastructure of a cell, which is often a requirement for proper function of a protein. Moreover, the natural environment can change protein properties and even its activity completely, making it difficult to put the individual properties of a protein into the bigger picture.

A great portion of the interplay and signalling between and inside cells is controlled by plasma membrane bound receptor proteins. Such signalling is directed by activator molecules (ligands) travelling inside an organism until recognized by a receptor protein residing on the plasma membrane of a cell. These receptor proteins are partly physically outside the cell, hence available for attachment of fluorescent labels or other chemically functionalized microscopic tools. Fluorescence techniques provide a unique way to study one protein species at the time. The fluorescent labelling of a particular protein requires a lot of effort in finding the right way to introduce labels, and labels have been created for a small but important subset of the proteome. For these proteins, the single-molecule tracking fluorescence microscopy technique makes it possible to observe their motion within living cells, and the technique is especially suitable for plasma membrane receptors. The motion of receptors is related to molecular interactions with these proteins. Characterizing the molecular interactions of proteins during their lifetime is a central topic in cell and systems biology and requires the development of new biophysical tools. In this thesis I aim to further advance imaging and analysis tools for single-molecule tracking microscopy, and apply these to the epidermal growth factor (EGF) receptor.

\subsection{Research aim}

Many proteins show a non-homogenous distribution in the plasma membrane. The exact nature and the role of the areas where proteins are apparently clustering are not yet completely understood. Clustering of proteins has been related to elusive nanometer scale cholesterol dependent lipid domains, called lipid rafts ${ }^{1-4}$. A wide range of literature reports suggests that many receptor proteins cluster within such domains $s^{5,6}$. The hindered motion of proteins within domains or clusters increases the chance of the protein to undergo other molecular interactions, such as with downstream signalling proteins ${ }^{7}$. Therefore the spatiotemporal organisation of receptor proteins has profound effects on the signalling network circuitries ${ }^{7,8}$. Misregulations in the signalling by receptors, and in particular tyrosine kinase receptors, are implicated in the development and progression of various human cancers, and are a widely studied target for drug discovery $^{9-11}$. Despite the developments, current molecular based anti-cancer drugs show limited response, and knowledge on the resulting molecular effects (e.g. clustering) on the plasma membrane is limited.

In this thesis, we focus on the epidermal growth factor receptor (EGFR) family called ErbB or HER, a class of four receptors (ErbB1-4). The ErbB family receptor proteins are prototypical for tyrosine kinase receptors, and are also said to cluster into small domains. The estimation of the 
size of these domains range however from 10 to $200 \mathrm{~nm}^{12-18}$, which has profound implications on signalling amplification ${ }^{8,19,20}$. In addition, the EGF receptor undergoes a rather complex process before kinase activation comprising a ligand induced conformational change and allosteric dimerization to halt the auto-inhibitory kinase activity ${ }^{21-23}$. The exact nature of the relations between receptor activity, the role of ligands, conformations, dimerization, and higher order cluster formation, as well as the dynamic behaviour of receptor movements across the plasma membrane, remains to be clarified by improved experimental methods and advanced analysis tools.

The progress of our molecular understanding of signalling complexes on the plasma membrane is hampered by the difficulty to directly visualize the protein dimers and small clusters in live cells. Until recently, fluorescence microscopy techniques were restricted to a resolution down to 200 $\mathrm{nm}$, the diffraction barrier that limits the resolution of visible light microscopy. The barrier was circumvented by cleverly making use of single fluorophore localization in so called superresolution fluorescence microscopy techniques, such as photoactivated localization microscopy $(\mathrm{PALM})^{24}$ and stochastic optical reconstruction microscopy (STORM) ${ }^{25,26}$. Although these techniques brought the resolution down to $20 \mathrm{~nm}$, they are not applicable in living cells on the short time-scales that clusters and dimers are believed to exist (milliseconds to a few seconds). Electron microscopy (EM) methods have also been used to investigate the clustering of EGFR ${ }^{27}$, but is also not applicable in living cells. These visualization techniques therefore cannot provide information on the dynamic motion of receptors. Single-molecule tracking is more suitable for this goal. This technique also allows the observation of dimerization and higher-order clustering in live cells, such as by correlated motion analysis ${ }^{28,29}$. Alternatively the diffusion exhibited by each receptor protein can be related to the cluster size $e^{30,31}$. Analysis methods to distinguish different motion modes from trajectories and relate these to the cellular context are however still immature. Different diffusion populations (states) are hard to distinguish due to the broad spread of step sizes in a diffusive system. Classification of the diffusion states within trajectories is also hampered by their short lifetimes, and the limited number of localizations available before current fluorescent labels suffer from photobleaching.

The research aim for this thesis is to further develop tools for studying the inhomogeneity of receptor motion at the plasma membrane with the possibility to relate this motion to the cellular context. We aim to develop tools that provide information about the molecular behaviour of proteins despite the current limitations in fluorescent labels and number of localizations within the duration of a diffusion state. We will test and apply these tools to ErbB family proteins. To achieve this aim, we have identified several objectives. First, the imaging of receptor motion in live cells requires the attachment of a fluorescent label to the receptors. We will identify suitable fluorescent labels for single-molecule tracking of membrane receptors. Therefore we will utilize an existing method to fluorescently label plasma membrane proteins using a universal protein tag, and further advance this labelling method to the single-molecule level. Optimal substrates with different emission spectra can be used to obtain dual-colour labelling of SNAP-tag receptors at the single-molecule level. When the trajectories of a single protein species are recorded in multiple colours, they can reveal the kinetics of homo-dimerization interactions by co-movement of the labelled molecules ${ }^{28,29}$. Next we will develop nano-scale visualization and diffusion characterization tools to quantitatively assess the motion of receptors within the plasma membrane. We will apply the tools developed on trajectories of EGF receptors recorded using the advanced labelling method, and will relate the diffusion of the receptor population to their dimerization state under different agonistic and antagonistic conditions. These receptor antagonists are currently used as drugs in anti-cancer therapies. Finally, we will move forward to induce a controlled activation of EGF receptor using existing methods to functionalize AFM tips with ligands. The use of a scanning probe microscopy approach allows us to present EGF ligands 
to specific locations on the plasma membrane of the cell while we monitor receptor motion using single-molecule fluorescence microscopy.

\subsection{Outline of the thesis}

In this first chapter we will introduce what is currently possible to image and probe in this nanoscale world of interactions with plasma membrane bound receptor proteins in living cells by fluorescence microscopy. Since we focus on the ErbB protein family, we will first provide a brief introduction on the current molecular understanding of the ErbB receptor family necessary to understand the research in this thesis. Next we will introduce the concept of single-molecule tracking fluorescence microscopy, and we will discuss the requirements for fluorescent probes to be used in this technique. Besides visualization of protein interactions, we took a next step in single-molecule research on live cells, and performed proof of principle experiments for locally controlled manipulation of the signalling state of receptors. To understand the context of the development and the AFM-based methodology, we will finish this chapter by a brief review of single biomolecules manipulation tools and the chemistry necessary to attach biomolecules to an AFM tip as used in single-molecule force spectroscopy by AFM.

Chapter 2 describes the requirements of a single-molecule sensitive fluorescence microscope to track single proteins in live cells. Tracking of single protein requires short acquisition periods to achieve high localization accuracies, and such short acquisition powers are only possible by higher illumination powers than available in commercial microscopes. We therefore designed and implemented a custom built microscope, and provide a thorough characterization of its performance. To construct protein trajectories from the recorded image series, tracking software is available, although such software is still in a development stage. We shortly describe a few contributions we made to further improve one of the most promising software packages available. The chapter finishes with an overview of current analysis methods used to characterize the motion of proteins in live cells from the trajectories obtained after tracking.

In Chapter 3 we describe our framework composed to perform a classification of dynamical diffusion states from protein trajectories obtained using single-molecule tracking microscopy. Diffusion state classification allows to determine, for each position within a measured trajectory, in which diffusion state a molecule most likely resides. The motion of membrane proteins can often be described by two dynamic populations of pure Brownian diffusion ${ }^{28,32,33}$, but classification is hampered by the broad distribution of stepsizes that are observed in Brownian motion, the limited localization accuracy, and the stochastic switching between states. Different analysis methods currently used to characterize the motion of proteins can be used in our framework. These analysis methods are mostly measures that provide a quantitative value to a segments of a trajectory, such as a relative confinement strength ${ }^{34,35}$, mean squared displacements ${ }^{36}$, or gyration $^{37,38}$. We tested the performance of these analysis methods in our framework by performing dynamic two-population Brownian simulation, and compared these to a maximum likelihood approach ${ }^{39,40}$ and a Bayesian approach ${ }^{41}$, which directly predict the diffusion states within trajectories. After each position in a trajectory of a molecule is classified to a diffusion state, a spatio-temporal map of diffusion states is obtained. These maps might show cellular zones or structures where protein interactions occur, wherever the diffusion of proteins decreases upon binding such structures. The chapter shows several examples of the possible applications of our framework for trajectories of EGFR.

Although proteins can also be fused to an autofluorescent protein (FP), they cannot match the photostability of small organic dyes ${ }^{42,43}$, limiting the timescale over which a protein can be tracked. Quantum dots are extremely bright and photostable, but they are considerably larger than most proteins themselves and require custom fabrication to be a monovalent label ${ }^{44-46}$. These effects affect the movement and artificial clustering of proteins ${ }^{47}$. More recent genetic protein tag 
techniques, such as SNAP-tag ${ }^{48-52}$, allow the specific labelling of proteins with small organic fluorophores in live cells. Although a few fluorescent substrates are commercially available, such substrates are not always suitable for use in single-molecule tracking microscopy due to high nonspecific binding or low photostability. Chapter 4 describes our survey of fluorescent substrates for the SNAP-tag. We developed measures for characterizing these properties at the singlemolecule level, and surveyed 6 green and 16 red excitable dyes for their suitability in fluorescence tracking microscopy.

In Chapter 5 we utilize the fluorescence labelling technique from the previous chapter to label EGFR and HER3 receptors, and characterise their mobility using the framework developed in chapter 3. We relate the altered motion of liganded and pre-clustered EGF receptors to its dimerization state using the well-established models for EGFR dimerization. Next we looked at the altered mobility when the receptor is targeted by the EGFR inhibitors erlotinib ${ }^{53}$, lapatinib ${ }^{54}$, and cetuximab ${ }^{55}$, which are used in current anti-cancer therapies. Combination therapy where multiple proteins are simultaneously targeted (e.g. EGFR and ErbB3) seems to have most promise for therapeutic success ${ }^{56,57}$, but there is limited or inconsistent knowledge on the molecular interactions and dimerization of EGFR and ErbB3 in the early events of EGFR targeting. For example, it is still an open question whether kinase inhibited EGF receptors are monomeric or whether they form homo- or hetero-dimers with or without ligands. Another unresolved issue is whether cetuximab is only preventing receptor activation by blocking the ligand binding site, or also sterically hinders the extracellular region of EGFR from making any conformational change to alternative conformations which might also result in dimerization ${ }^{58-60}$. In many different cancer cell types, the ErbB pathway can get overactive by different ways: overproduction of ligand, overproduction of receptors, or constitutive activation (due to mutation) of receptors. Each way leading to the same misregulation effect has to be treated by different therapies (different drug molecules influencing at a different point in the signalling network), so it is important to know why the ErbB system is overactive. This chapter describes our findings on the altered mobility of EGFR and ErbB3 when exposed to receptor antagonists in different cell lines.

Most chemical interactions with cells are only investigated on a bulk level. However, a locally applied stimulus can provide key insights to reveal elusive details on the signalling design principles of the cell ${ }^{8,61}$. Biomolecules can be attached to AFM tips to probe interactions with a recognizing surface or cells $s^{62-64}$. Whereas chemical force probe microscopy has been used to probe the energy landscape of proteins ${ }^{65}$, its use to locally actuate cellular effects has only seen the rudimentary combination with white light microscopy ${ }^{66,67}$. Clearly a much more advanced toolbox is anticipated when functionalised AFM tips are combined with the advanced optical microscopy methods developed, such as measuring FRET to monitor protein activity, or single-molecule tracking to monitor the altered mobility or observe dimerization of receptor protein. The AFM probes may present different stimuli to the receptors, such as activating ligands but also inhibitory antibodies. In Chapter 6 we show preliminary results of this next step in the single-molecule toolbox to study the biophysics of plasma membrane receptors. The attachment of small biomolecules such as ligands prove to be a difficult task, and we describe a devised single-molecule immunofluorescence assay to test the functionalization chemistry.

The findings of this thesis are discussed in the context of future perspectives in $\underline{\text { Chapter } 7}$, and is followed by a summary of the thesis. 


\subsection{Epidermal Growth Factor Receptor family}

Many reviews on the ErbB superfamily are available, e.g. ref. 9,23,68,69. This section contains a summary of information from these reviews essential for this thesis.

\subsubsection{Biological relevance of studying ligand-receptor interactions}

Molecular interactions between biomolecules are the fundaments of the chemical machinery which provide cells all the capabilities in performing their biological processes. Proliferation (cell growth), cell divisions, protein transcription, and many more cell processes, are examples of biological processes caused by the response of the cell upon stimulating signals. The receptor proteins are the first link in the system of signalling between biomolecules, and its activation leads to very specific activation or suppression of certain transduction pathways. Insight in interactions and distribution of receptors can therefore provide important information for understanding and intervening in tumour or tissue development, immunology, gene expression, and many more medical questions.

To systematically comprehend ligand-receptor bindings, parameters describing such systems are needed. Since protein signalling were believed to be mainly caused by simply associating or dissociating of two molecules, signalling could be described using the affinity or equilibrium constant $K_{a}$ which provides the molecular strength of (un)binding between molecules. Signalling networks consists of many proteins with positive or negative cooperativity and other feedback regulation loops. This complexity makes that the binding affinity $K_{a}$ is no longer seen as the only parameter important in describing properties of intermolecular binding and signalling. The parameters needed to fully describe the interaction properties between ligands and receptors are listed in a review by Robert et al. ${ }^{70}$.

For more information about the molecular building blocks of proteins, and cell regulation by receptor phosphorylation, please refer to Chapter 3 of the textbook "Molecular Biology of the Cell" by Alberts et al. ${ }^{71}$.

\subsubsection{Receptor Tyrosine Kinases}

An important class of proteins in signal regulation are the kinases, which catalyse the phosphorylation of (other) proteins. The phosphorylation might induce a conformational change which transforms it from an inactive to an active form. The phosphorylated amino chain can also form a target to be recognized by other downstream proteins of the signalling pathway. Kinase receptors can form a specific target to a protein by recognition (binding) of another domain of the target protein. For example, phosphorylated tyrosine side chains (the rest-group of this amino acid) are recognised by $\mathrm{SH} 2$ domains in proteins. Kinase proteins that are known to phosphorylate specifically tyrosine side chains are members of the tyrosine kinase family. The phosphate group can be actively removed again by phosphatase enzymes, but also has a chance to get removed due to thermal motion inside cells.

One of the receptor tyrosine kinases is the epidermal growth factor receptor (EGFR). This receptor is expressed in many tissues, but epithelial cells in particular depend strongly on this receptor for proliferation ${ }^{72}$. EGFR is also called ErbB1 as it is a member of the ErbB-receptor superfamily, which consists of four closely related receptors. The other members are called ErbB2, ErbB3 and ErbB4. All the ErbB receptors are expressed in many different tissues and play a crucial role in cell proliferation and differentiation. The normal function of ErbB receptors and their ligands is a mediatory role for communication between cells in organogenesis, the organ development period, and adulthood ${ }^{68}$.

The cognate ligand for EGFR is epidermal growth factor (EGF), while other ligands structurally similar to EGF, like the NRG family, bind other members of the ErbB family receptors ${ }^{72}$. The ligands are released from cells by cleavage from a large transmembrane precursor. The expression 
of the precursor and the ligand release are highly regulated by for example Ras proteins and steroid hormones ${ }^{6}$. EGF-like ligands can bind one type or a broader range of ErbB receptors. EGF and TGF- $\alpha$ bind to ErbB1. ErbB2 (HER2) lacks a ligand binding site ${ }^{73}$ and is the preferred heterodimerization partner for EGFR ${ }^{74}$ and is involved in many cancer types ${ }^{75,76}$. ErbB3 (HER3) has another ligand (NRG1) than EGFR and is a pseudo-kinase ${ }^{77,78}$, and has recently become much more in focus as a target in cancer therapy ${ }^{11,79}$. The ErbB3 receptor is believed to have much reduced kinase activity and can only be trans-activated (phosphorylated) by other ErbB receptors through dimerization. All NRGs bind to ErbB4 (HER4), a receptor which is less well studied. Since the activation of EGFR is involved in various cell processes, such as cell survival, cell growth, cell division, and differentiation of cell type, the different activation methods and stimulation durations are an important research object to gain insight into cellular communication and defects in tumour cells.

\subsubsection{ErbB receptor dimerization}

Ligand binding allows two receptors to dimerize through an extracellular conformational change from the tethered to the extended form. The extracellular regions of all ErbB receptors are composed of four subdomains, L1, CR1, L2, and CR2, or more commonly called domain I, II, III, and IV respectively. The $\mathrm{L}$ domains consist of repeating leucine-rich motifs and the CR domains are cysteine-rich. The ligand recognition site of the receptors are located in domain I and III. The kinase domains are intracellular and these kinase domains have an enzymatic function in phosphorylating (other) protein members of the ErbB family. In the ligand-bound conformation the kinase of the homo- or hetero-dimerization partner of the receptor cross-phosphorylates the characteristic tyrosines in the cytoplasmic tails, as shown in Fig. 1.1.

Domain II of the extracellular part of the ErbB receptors serves as the dimerization arm to allow the receptor to form a dimer with another receptor ${ }^{23}$. As a dimer, one or more intracellular tyrosines are phosphorylated. Whereas most RTKs reach full activity following transautophosphorylation of their kinase domains within a dimer, ErbB receptors form asymmetric dimers that allosterically activate the kinase domain ${ }^{80}$. This means that the kinase domains do not phosphorylate each other, but that binding of a different domain leads to activation of the kinase domain. ErbB receptors are an exception to most other receptor types in the RTK family, as they do not require trans-phosphorylation, but use allosteric activation for the tyrosine phosphorylation ${ }^{23}$. The phosphorylation in heterodimers has not been studied in great detail. It has also been suggested that the heterodimers are actually two homo-dimers forming a hetero-tetramer ${ }^{81}$.

Numerous studies on the distribution of EGFR indicate that the receptor can pre-dimerize in ligand-free homo- and heterodimers ${ }^{82-85}$. The pre-clustering was shown to be regulated under physiological conditions by the expression level of the receptor ${ }^{86}$. Preformed unliganded EGFR dimers are only found when the receptor was overexpressed ( $>300,000$ receptors), or at subphysiological temperatures ${ }^{87}$. Pre-formed dimers are a method to increase the sensitivity of EGF, since such pre-clustering clearly has profound effects on the time required for two receptors to dimerize and activate, as the receptors are already together to transform into a signalling dimer. The result is rapid receptor signalling, also at low amounts of EGF, due to a positive cooperativity in the (rapid) binding of a second EGF due to the altered affinity of ligand to the receptor ${ }^{88}$, and possibly due to pre-formed hetero-dimers with ErbB2 which does not need a ligand to activate dimer partners.

Number and brightness experiments showed that upon EGF stimulation EGFR goes from a mainly monomeric state to an almost complete dimerized state as expected; simultaneously ErbB2 residing in macrocluster goes from large homoclusters to homoclusters containing less ErbB2 as they form heterodimers with EGFR ${ }^{86}$. 


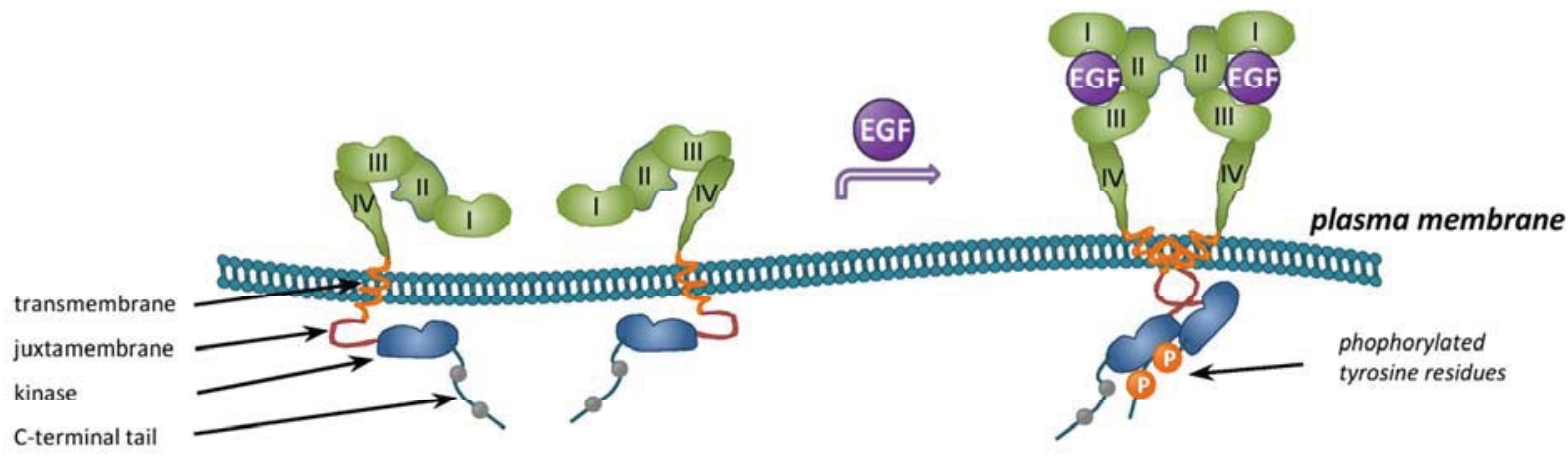

FIGURE 1.1 Schematic image of the structure of EGFR. On the left the inactive tethered or closed conformation is shown, which is the normal resting conformation when no ligand is bound. On the right the extended (untethered) conformation of EGFR in a dimer formation is shown. When a ligand binds, the receptor undergoes a conformation change from the tethered to the extended conformation, such that its dimerization arms in domain II can induce dimerization. The ligand induced dimerization allows the allosterically activated kinase of a receptors partner to cross-phosphorylates the tyrosine residues in the cytoplasmic tail of the receptor.

\subsubsection{ErbB signalling pathway}

Since the ErbB receptors are trans-membrane proteins, all but ErbB2 require an extracellular ligand as a messenger for receptor activation. After ligand-induced activation of the tyrosine kinase of the receptor, the sequence of the phosphorylated tyrosine residues becomes a docking site for Src homology 2 (SH2) and phosphotyrosine-binding (PTB) domains ${ }^{89}$. Not all ErbB members interact with common adaptor proteins for the receptor, such as Grb2 and Shc. ErbB3, which lacks kinase active domains, cannot interact with Grb2, but it can with Shc. The interaction with adaptor proteins is known to last for at least two hours after stimulation of ErbB1 with a saturation dose of EGF ligand ${ }^{89}$.

An important target of the actuated system is the Ras protein (a GTPase) via the Grb2 and Sos adapter proteins ${ }^{89}$. Since this triggering is a short lived process, the signal is forwarded to the mitogen-activated protein (MAP) kinase module, the downstream kinases of this module causes gene expression in the nucleus and DNA transcription to regulate protein expression. The MAP kinase module consists of three kinase proteins, the last one is called MAP kinase (MAPK, also called Erk) whose phosphorylated form is commonly used in immunochemical assays as a measure for EGFR activation. The preceding kinase is called MAP kinase kinase (MAPKK, also Mek), and the one preceding that kinase MAPKKK (also Raf) which is activated by the Ras protein. Interestingly the period that MAP kinases are activated results in different cell behaviour. For instance in neural precursor cells the activation of the MAP cascade by EGF leads to an Erk MAP kinase activity peak of 5 minutes resulting in cell division, while activation by another ligand via other receptors leads to prolonged Erk MAP kinase activity for many hours resulting in cell differentiation. Other pathways associated with EGFR activation are the Phosphoinositide 3kinase (PI3K) pathway which are a class of kinases which have been linked to a diverse group of cellular functions and the phospholipase C (PLC)- $\gamma$ pathway ${ }^{71}$.

The signal output of ErbB family includes at least cell division, migration, differentiation, adhesion, and apoptosis. The output depends on the cellular context, which ligand activated the receptor, and what ErbB dimer type was formed. Homo-dimer formations are less mitogenic (cell division) and transforming (genetic alteration to influence proliferation and cellular differentiation) than the hetero-dimer combinations; ErbB2 heterodimers are the most potent signalling complexes.

After ligand binding, EGFR receptors cluster over clathrin-coated regions on the plasma membrane, to form endocytic vesicles (early endosomes) and are either recycled or sorted to late 
endosomes and lysosomes, before degradation. Receptor ubiquitination through a Grb2-Cbl ubiquitin ligase complex plays a role in the regulation of receptor internalization, which can be very rapid in the case of EGFR dimers, resulting in efficient down-regulation of the receptors ${ }^{90}$. The other ErbB receptors are more often recycled to the cell surface, and can affect the rapid internalization of EGFR. For example, the hetero-dimerization with ErbB2 leads to a reduced endocytosis rate of $\mathrm{EGFR}^{91}$. In this thesis we did not focus on this internalization and recycling process; reviews can be found elsewhere ${ }^{92,93}$.

\subsubsection{Membrane domains and the involvement of ErbB receptors}

The EGF receptor is non-homogenously distributed on the plasma membrane, and is organized as free monomers, dimers, and higher-order clusters in cholesterol-rich zones ${ }^{18,94}$ like many receptor proteins ${ }^{95-97}$. The numbers of receptors in individual clusters vary from one or two proteins to more than 100. This organization of receptors into nano-scale complexes is expected to enhance the sensitivity of receptors to changes in the concentration of ligand ${ }^{8}$. Stimulation with EGF shifts the distribution of EGFR in clusters to smaller and larger clusters, suggesting that ligand-bound receptors get confined to the clusters, and receptors in clusters undergo internalisation.

Several submicron sized membrane domains have been identified, such as lipid rafts, caveaolae and clathrin-coated pits. Caveolae and lipid rafts are zones rich in cholesterol and glycosphingolipids, and are involved in receptor signalling and endocytosis. Clathrin-coated pits are a subset of such lipid rafts, are rich in the caveolin protein in addition, form $50-100 \mathrm{~nm}$ invaginations in the cell membrane, and are the main site for EGFR internalization (early research on this mechanism is summarized in ref. 87). Interestingly, no colocalisation between GPI anchored proteins, known to reside in cholesterol rich regions, and unliganded EGFR is observed. Upon EGF stimulation, there is a significant colocalisation observed using FRET $^{18}$.

Transiently formed cholesterol enriched regions are believed to form nano-scale domains, reducing the mobility of proteins by aggregation of lipids and proteins in this domain. Although confinement is inherent when mobility is transiently reduced, these nanodomains are believed to promote oligomerisation of receptor proteins, resulting in the receptor confinement. The actin cytoskeleton, due to its membrane-associated F-actin strands, is thought to sterically interact with the cytoplasmic tail of transmembrane proteins. The actin creates microdomains in which proteins get confined, and the actin acts as fences and pickets within the membrane ${ }^{1}$. Also, the actin skeleton is influenced by the organization of cholesterol, possibly caused by dislocation of PIP2, an actin cytoskeleton regulator ${ }^{98}$. Therefore rafts could also cause micro-scale confinement due to the actin surrounding the raft.

ErbB receptor oligomerization in the context of membrane domains is still under debate. The oligomerisation of EGFR has been studied in detail by Hofman using anisotropy measurements ${ }^{99}$. The main conclusions from his work are that upon EGF stimulation EGFR goes from $40 \%$ predimer state to mainly higher order oligomers in nanoclusters and intracellular vesicles. Ligand induced dimerization was maintained for 10-20 minutes, after which receptors probably dissociated or degraded. For a kinase-dead receptor mutant, predimerization was still observed but no further clustering upon EGF stimulation was seen. Clustering was also no longer observed for a receptor mutant devoid of tyrosine residues. Whether this shows that dimerization is the result of kinase activity, as argued by the authors, remains unclear; it was not shown that kinase activity is also possible without dimerization, such that kinase activity may still depend on the allosteric phosphorylation facilitated by dimerization. In kinase dead mutants these allosteric connections are not possible anymore. However, the kinase domain itself is a necessity for dimerization. Furthermore, artificially induced dimerization or oligomerisation of the receptor did not result in receptor actuation, which is in contrast to other reports ${ }^{100}$. Artificial clustering also did not yield a stronger activation signal (biochemical readout) upon EGF stimulation. Internalisation rates after EGF stimulation were dramatically increased when the receptors were 
artificially pre-dimerized. Certainly, the number of receptors is still the same, and only the timeframe of activation will be faster simply since receptors do not need the time to find each other like in the case of pre-dimers.

FIDA, a mathematically equivalent approach to brightness analysis, was used to study EGFR ${ }^{101}$. It found that cyclodextrin, which depletes cholesterol, induces the assembly of monomers into (higher order) oligomers, while cholesterol-loaded cells made pre-formed dimers become monomers. Therefore, the net effect of cholesterol depletion was to increase clustering of the EGF receptor. Since depleting cholesterol leads to fewer available rafts, EGF receptors residing in these rafts can only concentrate in the fewer rafts available. It should be noted that FIDA only takes the mobile population of receptors into account, hence when immobile receptors are differently clustered compared to the mobile population, or when clustering leads to immobility, the analysis may neglect a significant population.

By tracking EGFR and ErbB2 receptors, the diffusion speed of EGFR was found to be slower compared to ErbB2 ${ }^{94}$. Upon latrunculin A treatment, which inhibits the filament end of G-actin, EGFR speeds up to the same diffusion value of HER2 and the confinement strength is reduced. Actin disruption showed no significant change in confinement strength for ErbB2. This implies that the actin skeleton helps to form immobile clustered EGFR but does not interact with ErbB2. Cholesterol depletion led to an increase of $10 \%$ to $80 \%$ of the total EGFR population to stay confined in nano-scale domains, and the residence time went up from 1.4 to 2.2 seconds. The nano-scale domain confinement was ascribed to the normal or cholesterol enriched membrane, while the larger scale confinement in cholesterol depleted domains was ascribed to actin. Although reasonable, these conclusions were based on a time-averaging analysis of long time-lag MSD curves ${ }^{102}$, which are not reliable ${ }^{36}$ (and Chapter 2). Much more dynamic transitions between different membrane zones may play a role as well.

\subsection{Single-molecule Tracking Microscopy}

It remains a dream to be able to follow a protein and its interactions as the protein travels through the cell during its lifespan. Single-molecule fluorescence imaging already allows one to track a protein for a short period of time and record its trajectory ${ }^{28,103,104}$. Trajectories of proteins in a living cell contain valuable quantitative information on its interactions with its microenvironment. The protein molecule often interacts with other molecules resulting in transient slowed diffusion by cytoskeletal or other nano-scale membrane structures ${ }^{28,32,33,104-107}$. To know the proteins interactions and the kinetics of the interactions is important to understand the underlying signal transductions and the cellular signal regulatory ${ }^{7}$. The compartmentalization of the plasma membrane plays a key role in the observed dynamics of protein $s^{20,108}$. Therefore the study of the spatiotemporal dynamics of proteins is important to confirm hypotheses from mathematical modelling of protein clustering ${ }^{19,20,109,110}$, and to detect whether certain membrane structures are related to receptor motion (with tools developed in Chapter 3). Single-molecule tracking microscopy is a unique method that can provide information about this highly dynamic behaviour, and is especially well suited for application on cell membrane bound receptors, the cellular region accessible for total-internal reflection fluorescence (TIRF) microscopy.

Using a single-molecule sensitive microscopy it is possible to observe single fluorescently labelled molecules or small clusters over time, on a timescale down to the order of a millisecond ${ }^{104,111}$. By fitting the centre positions of single spots in fluorescence video stacks, it is possible to find the location of the molecule with a localization accuracy of typically 20 to 40 nanometers, which is well below the diffraction limit. Localization is only possible when the number of labelled proteins is so sparse that only individual (non-overlapping) spots are visible. Tracking algorithms then link the positions in each frame to compile the trajectory travelled by a single protein (Fig. 1.2). To detect molecules in a microscopy recording, and track them to obtain 
protein trajectories is nontrivial. Significant effort is being put into optimizing the tracking process, starting from rudimental methods to advanced statistical approaches using maximum likelihood ${ }^{28,104,105,112,113}$. The result is a list of trajectories of the proteins, which can be used for further analysis.

Single-molecule tracking has several clear advantages over conventional light microscopies. While conventional light microscopy techniques are restricted in spatial resolution by the diffraction of light, single-molecule tracking has a higher resolution, as it is possible to locate the centre of a single spot with a precision well below the diffraction limit of light. Also the timeresolution is much higher compared to FRAP measurements to determine diffusion coefficients, as a couple of frames (1-40 ms typically) are sufficient to detect transient zones with altered motion. Furthermore, single-molecule techniques yield the complete distribution of a phenomenon, contrary to the ensemble-average of bulk measurements which cannot identify different subpopulations. Although in single-molecule tracking only images are made of a subset of receptors, this should not be a problem due to the ergodic principle. We can extend the measurement over time to compensate for the inherent presence of dark receptors.

To allow optical microscopy of single proteins in a living cell, the common approach is to attach a fluorescent label to the protein species being studied. While other probes are available to attach to the protein of interest, such as colloidal gold particles and micrometer sized polystyrene beads, these probes are an order of magnitude larger than the protein itself, which can cause artefacts and steric hindrance. Fluorescent labels are usually of the same size of the protein, or smaller in the case of organic fluorophores ${ }^{114}$.
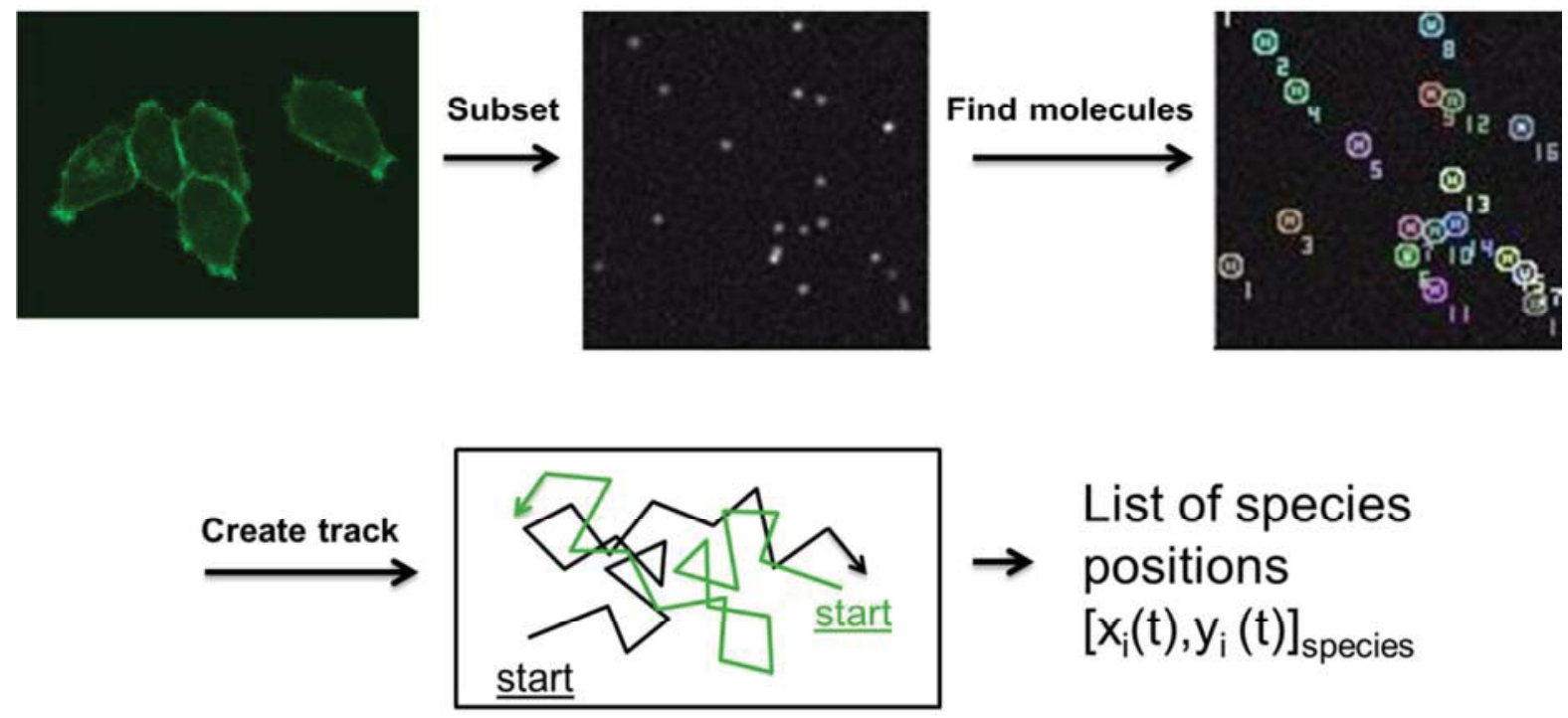

FIGURE 1.2 Single-molecule tracking requires that only a subset of the receptor studied is labelled, such that the number of labelled proteins is so sparse that only individual (non-overlapping) spots are visible. By fitting the centre positions of single spots in fluorescence video stacks, it is possible to find the location of the molecule and link the positions in each frame to compile the trajectory travelled by a single protein.

\subsubsection{Direct visualization of protein interactions}

With a one-colour microscopy setup it is possible to study the diffusion behaviour of single molecules, which may answer fundamental questions about the (origin of and control over) motion of the single receptors. With a two-colour microscopy setup, it is possible to observe the motion of single receptors of one or two species in two colours, which can also answer questions about molecular interactions. For example, it allows the direct observation of homo- and hetero- 
dimerization of single receptors when they are labelled with different colours ${ }^{29,115}$. The observation of two receptors labelled in one colour is not possible due to the diffraction limit (Fig. 1.3).

Other techniques to measure oligomerization of receptors, are fluorescent (number and) brightness analysis ${ }^{116}$ and image correlation spectroscopy (ICS) ${ }^{117,118}$, yielding information on the scale of the diffraction limit. Dimerization is perhaps better probed by FRET techniques ${ }^{119}$, using hetero-FRET by FLIM, and homo-FRET by measuring the anisotropy, which in both cases yield information on the length scale of the Förster radius $( \pm 4-8 \mathrm{~nm})$. It is believed that this radius only probes dimerization, as the distance in too short for FRET between higher order oligomerisation and clustering. However, dimerization cannot be revealed in all experimental conditions by FRET as we experienced. The lack of observed FRET can be related to the two fluorescent labels which cannot be attached to the receptor such that they get within the Förster radius upon dimerization. Furthermore the concentrations of the donor and acceptor fluorophores need to be carefully optimized. Single-molecule tracking is an additional technique to probe protein interactions, and can do this at super-resolution at the single receptor level in a spatiotemporal fashion. This adds the possibility to measure the lifetimes of the dimers formed.

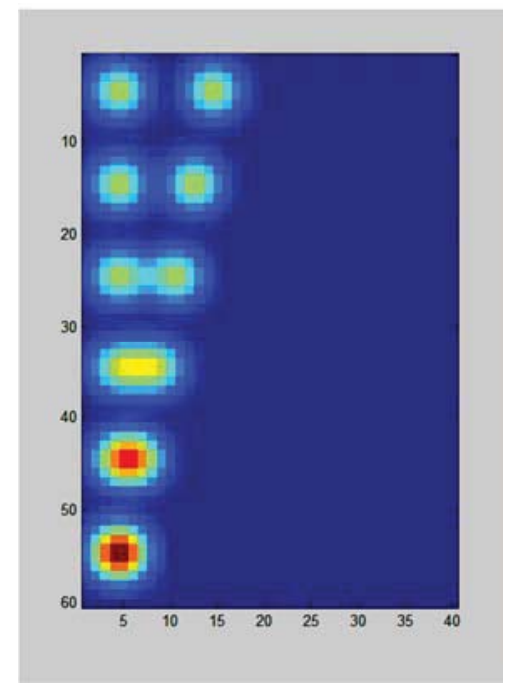

FIGURE 1.3 When two Gaussian shaped objects come close together, they start to overlap and form another Gaussian shaped object. The two objects become indistinguishable from each other when the distance gets smaller than the diffraction limit. According to the Rayleigh criterion, this distance equals $0.61 \lambda / N A$.

\subsection{Fluorescent probes}

Fluorescent labels have gone through stages of advancement. The autofluorescent proteins (FPs) have provided a wealth of possibilities and new knowledge. Yet a disadvantage of the FPs is that most are not overly bright and photostable. They are also incorporated in relatively large beta barrels ( $27 \mathrm{kDa}$ for GFP in monomeric form) which can interfere with the protein function when the FP is not separated from the protein with a long enough linker of amino acids. Another disadvantage is that almost all FPs tend to form weak dimers or tetramers, which consequently brings the protein of interest artificially into oligomers. Furthermore fluorescent proteins often experience complicated photo-physics, and suffer more from blinking and photobleaching compared to other fluorescent labels. These disadvantageous properties make FPs less suitable for demanding spectroscopic methods and prolonged imaging.

Organic dyes, such as fluorescein and rhodamine, can be more photostable and therefore offer more photons before they bleach ${ }^{120}$. Organic dyes are also relatively small (usually under $1 \mathrm{kDa}$ ), which minimize the interference with protein function. The small organic dyes rarely perturb the protein function as possible steric hindrance for smaller molecules is less likely ${ }^{121}$. The availability of organic dyes with (far) red-shifted emission is much larger than for fluorescent proteins ${ }^{122}$, with infra-red emitting fluorescent proteins just recently discovered ${ }^{123}$. In experiments using live cells this is important, since blue light causes more cell damage than light of higher wavelengths. 
Infra-red emitting fluorophores can also be used for imaging of tissue in vivo, and allow optical xenografts, or diagnostic applications in the clinic. Especially molecular imaging agents that can be dual labelled with a nuclear reporter (radioisotope) and an infra-red fluorophore are a promising tool for providing both the diagnostic imaging capability with PET or SPECT, while subsequently providing intraoperative guidance with near-infrared fluorophores ${ }^{124,125}$.

Quantum dots have excellent properties concerning photostability and brightness, and blinking can be significantly suppressed by using Trolox ${ }^{126}$ or a special layer surround the core of the quantum $\operatorname{dot}^{127}$. However, they are larger than proteins themselves which might sterically hinder (blocking molecular interactions between molecules due to the physical presence) the movement and interactions of the protein ${ }^{128}$. To study diffusion behaviour by for example fluorescence correlation spectroscopy (FCS) or single-molecule tracking (SMT), quantum dots should be used with caution as they might artificially slow down diffusion of the protein of interest. Also for FRET based studies, quantum dots are not useful due to their large size.

To conclude, small and photostable organic fluorophores would be the appropriate choice for studies on the diffusion of biomolecules. Labelling of the protein with a photostable visible autofluorescent protein (FP) such as mCitrine, or with an organic fluorophore via an enzymatic tag or antibody might minimize steric hindrance.

TABLE 1.1 Comparison of different fluorescent probes for labelling of protein for various applications.

\begin{tabular}{|lll|l|}
\hline Size / Weight & Fluorescent proteins & Organic dyes & Quantum dots \\
& $3 \mathrm{~nm}$ & $\begin{array}{l}1 \mathrm{~nm} \\
1 \mathrm{kDa}\end{array}$ & $\begin{array}{l}20-40 \mathrm{~nm} \\
>30 \mathrm{kDa}^{129} \\
>400 \mathrm{kDa}^{127}\end{array}$ \\
\hline Labelling & Genetic fusion & $\begin{array}{l}\text { In vitro to side residue } \\
\text { peptide / antibody } \\
\text { In vivo binding to cell } \\
\text { compartment or to } \\
\text { genetically fused } \\
\text { protein tag }\end{array}$ & $\begin{array}{l}\text { In vitro streptavidin / } \\
\text { antibody conjugation }\end{array}$ \\
& & + & \\
\hline Brightness & $-/+$ & $+/++$ & ++ \\
\hline Photostability & $-/+$ & $+/++$ & ++ \\
\hline Specificity & ++ & $\begin{array}{l}\text { Protein, lipid, DNA, } \\
\text { peptide interactions } \\
\text { and dynamics in cells } \\
\text { and in vitro }\end{array}$ & $\begin{array}{l}\text { Protein, DNA and } \\
\text { peptide interactions }\end{array}$ \\
\hline Applications & Protein interactions \\
& $\begin{array}{l}\text { and environment in } \\
\text { cells }\end{array}$ & & \\
& &
\end{tabular}

\subsubsection{Motivation of probes used in this thesis}

Various protein-tag labelling strategies have been developed recently, which allow specific labelling of recombinant proteins with an epitope tag by small organic fluorophores within live cells ${ }^{121}$, such that prolonged imaging and tracking of proteins is possible. This strategy can certainly be used for labelling of proteins on the cell membrane of live cells, but also for cytosolic proteins when a membrane permeable label is used. As the protein can be fused with a highly specific tag, the specificity of labelling is increased compared to immunostaining, and is also useful when a high-quality antibody to target the protein of choice is not available. One of the genetic protein tag techniques developed is the SNAP-tag ${ }^{48-52}$, which allow the specific labelling of proteins with small organic fluorophores in live cells. We confirmed the high specificity of labelling SNAP-tag fused to EGFR-mCherry with an organic dye (Fig. 1.4), and later by investigations at the single-molecule level (Chapter 4). Also the size of the labelling complex is 
much smaller (1-25 kDa) compared to immunolabelling, solving the issues related to steric hindrance and slowed diffusion. Besides these specific advantages, these strategies can be advantageous in general, as one only has to prepare a plasmid once, after which one can label the fusion protein with a wide choice of dyes with different properties. For example, switching from a green emitting dye to an infra-red emitting dye is obtained by only one labelling step.
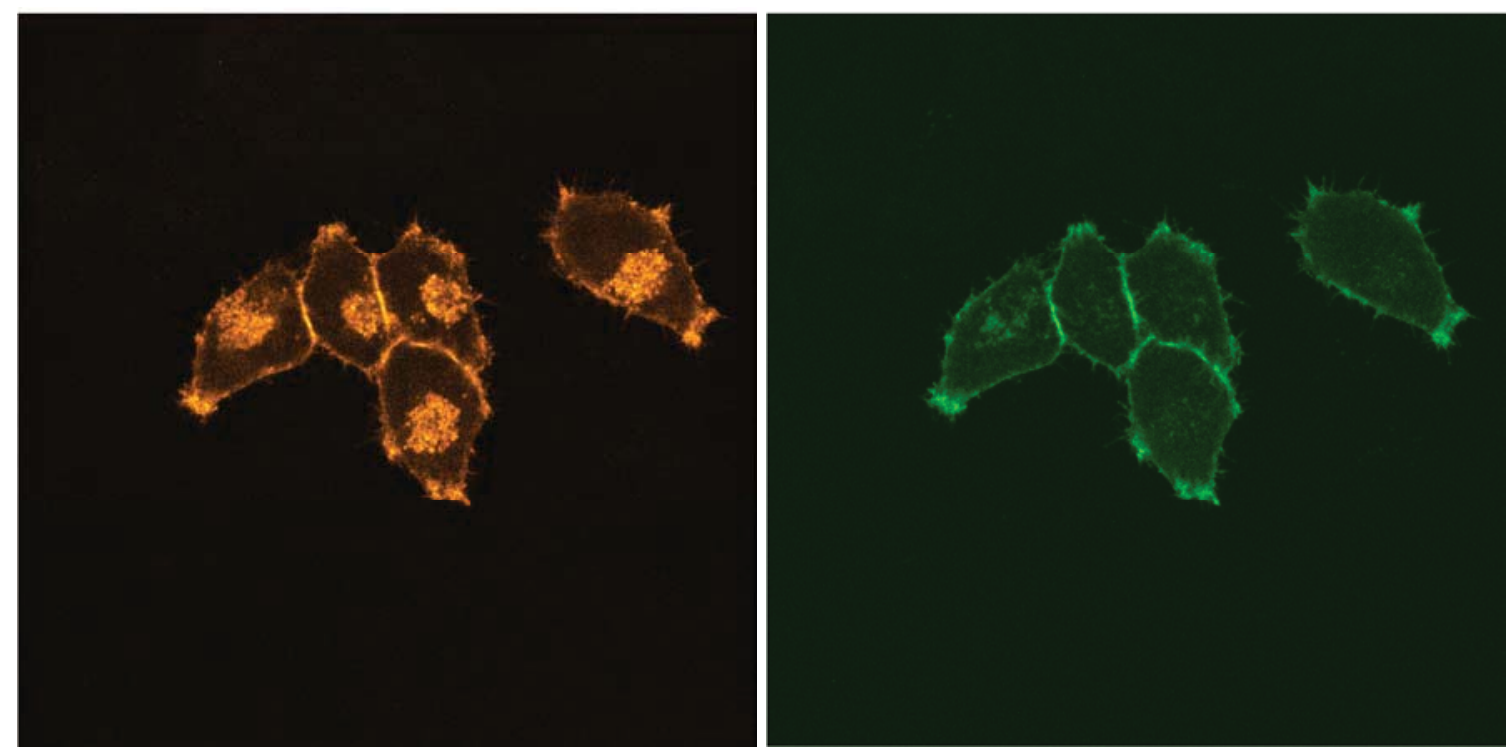

FIGURE 1.4 Confocal recordings of HeLa cells expressing SNAP-ErbB1-mCherry (left) labelled with BGAlexa488 (right) confirms the specificity of the SNAP-tag labelling strategy. We see that only membrane bound receptors are labelled by the substrates. Image size $150 \times 150 \mu \mathrm{m}$.

\subsection{Controlled receptor activating techniques}

To understand the local actuation methodology used in Chapter 6, in this section we provide guidance in what is currently possible in nano-scaled controlled probing of interactions between single biomolecules. We focus on the use of functionalised AFM tips and their preparation. A good review of various techniques for probing molecules one by one is given by Bustamante et al. ${ }^{130}$. Here we will discuss only manipulating (force exerting) and not solely probing techniques, such as flow chambers ${ }^{70}$ or functionalised substrates. The techniques that are able to exert (small) forces on molecules in order to guide them toward a binding spot can usually measure the binding force as well. In comparing the techniques, it is useful to compare the forces possible to exert and measure, the lateral resolution and stability, and the extent to which the natural environment (in vivo) of the sample studies is preserved.

\subsubsection{Optical and magnetic tweezers}

A strong external field can be used to influence the movement or measure the force of single molecules and interactions. The external fields being used nowadays for this purpose are photonic and magnetic fields. Usually photonic fields do not act directly on the molecule of interest, but typically on a glass, polystyrene or golden bead coupled to the molecule of interest. Most biomaterials have very low magnetic susceptibility, so for magnetic studies there is always a need to couple magnetic particle to materials of interest. The different fields differ mainly by the maximum possible force that can be exerted and the accuracy of control.

Optical tweezers were invented in 1970 and further pioneered by Ashkin ${ }^{131}$. The concept of using the momentum of infra-red photons to exert forces on small particles to construct an optical tweezer, has been reviewed numerous times in the past, see e.g. ref. 130,132. Principally a relatively 
large amount of radiation pressure (photon momenta) is obtained with a laser beam focussed by a high numerical aperture objective, to levitate an object with a distinct refractive index (usually a transparent spherical bead). An equilibrium position for the bead is found in the applied radiation gradient close to the centre of laser focus, and every perturbation from this position will result in a restoring force proportional to the perturbation in analogy to a mechanical spring. Magnetic tweezers do not produce an equilibrium position, but work similarly by applying a constant force due to a magnetic field acting on a strong paramagnetic bead.

Optical tweezers are very sensitive in force and displacement measurements: about $1 \mathrm{pN}$ and 1$10 \mathrm{~nm}$, respectively with high time resolution (down to $100 \mu \mathrm{s}$, usually a few milliseconds) ) $^{33,130}$. The lateral resolution can be improved to angstroms with advanced high end techniques ${ }^{33,133}$. Measurements can be done on living cells and surely on model systems. With typical bead sizes of $1-3 \mu \mathrm{m}$ the stiffness of the optical trap is 0.050 to $0.50 \mathrm{pN} / \mathrm{nm}^{132}$. The possibility to manipulate one bead (with a certain number of molecules under study attached) at a time is an advantage of optical tweezers over magnetic tweezers, since the optical radiation can (and is) focused on a single bead.

Magnetic tweezers are more limited in lateral resolution (down to 10nm) and time resolution (around 30ms), and are also less sensitive in force measurements than optical tweezers, but are very accurate in the applied force which can range from as low as $10 \mathrm{fN}$ to $200 \mathrm{pN}^{33,133}$. Magnetic tweezers can, in addition to a linear force, also apply or measure a controlled torque by simply rotating the magnetic field. Depending on the properties of the paramagnetic bead the applied torque can be as high as $1,000 \mathrm{pN} / \mathrm{nm}^{133}$.

There are also several limitations to the use of external field manipulators in biologic systems. The major disadvantage of optical tweezers is the damage by the high radiation from the focused laser (photodamage) to fragile biological molecules ${ }^{130}$. This limits the time frame in which measurements can be done on living cells, especially when high forces are needed, because the cells will be damaged after a prolonged or intense optical radiation. Secondly, beads are required to have a size of at least one wavelength in diameter because for smaller beads the trapping force scales down with the third power of the bead radius ${ }^{130}$. The need of such a huge bead relative to the molecules under study might influence the natural diffusion properties.

Although magnetic tweezers can apply high forces without the disadvantage of photodamage to the system, they are theoretically limited in sensitivity (due to inherent electron spin fluctuations), and are not readily able to provide full control in three dimensions since the forces from the magnetic field are restricted. Magnetic tweezers are therefore most suitable for measuring molecular processes that require the measurement or control of both force and torque.

\subsubsection{Atomic Force Microscope}

The so far mentioned techniques perform well in measuring molecular forces, however they have limited capabilities in the exact control of stimulating biomolecules. An atomic force microscope (AFM) can not only measure weak forces but can also easily move the tip with its piezocontroller in space, e.g. to scan the surface of a sample. In this way, it can also be operated to move and guide a molecule very precisely, thereby acting as a tool for an exact control of the position of biomolecules. AFM is a well-established instrument in biophysics, and good reviews are available for theoretical aspects ${ }^{134}$, as well as for biophysical applications ${ }^{135}$.

AFM can apply and measure large forces, in the range of 10 to $10,000 \mathrm{pN}$, which is sufficient to monitor even individual bonding forces when soft cantilevers $(<0.10 \mathrm{~N} / \mathrm{m}=100 \mathrm{pN} / \mathrm{nm})$ are used $^{70}$. The relative high stiffness of soft cantilevers $(10-100 \mathrm{pN} / \mathrm{nm})$ still limits the maximum force that can be applied, and a force resolution limit of 5-10 $\mathrm{pN}^{133}$. Typical scanning ranges are 5150 micrometers with about $1 \mathrm{~nm}$ precision (depending on the piezotube and piezostages used). The precision is not as good as with external field methods (optical and magnetic tweezers), but the AFM can measure and apply much higher forces ${ }^{136}$. This technique is therefore well suitable to 
measure and overcome individual ligand-receptor recognition forces which are found to be around $50-250 \mathrm{pN}^{137-140}$.

\subsubsection{Chemical force microscopy: proteins on a tip}

Single molecule force spectroscopy (SMFS) can be used to gain insights into binding strengths and kinetics $^{70}$, and to localise receptors on cell membranes when the molecular adhesion force is being mapped ${ }^{141}$. AFM is capable to sense chemical intermolecular interactions instead of the topography by functionalising the cantilever tips, which is called chemical force microscopy (CFM) or recognition imaging ${ }^{142}$. By attaching specific functional groups, such as compounds that interact (bio)chemically with the sample, the shape of the approach-retraction force curve contains a lot of information on the specific binding force being probed; these forces are reviewed in ref. 143.

The first receptor-ligand force spectroscopy experiments by AFM were performed in 1994 by Moy, Gaub, Lee et al. ${ }^{140,144}$. These experiments were performed with a directly coated AFM tip, or a functional glass bead attached to the tip. Although this methods results in a high amount of functional molecules on the tip, near single molecule interactions were possible due to the small amount of molecule that were physically oriented to allow a chemical interaction. Still it was not ideally suited to separate specific from unspecific ligand bindings onto cellular compounds, mainly because of parallel breakage of multiple bonds. In 1996 a new method was demonstrated by Hinterdorfer et al. ${ }^{139}$ who used a synthesized polyethylene glycol (PEG) spacer between the cantilever tip and the ligand, which prevents unspecific interactions. The next section outlines methods involving such spacers for a specific functionalization of AFM tips.

\subsubsection{Spacers}

The ideal single-molecule probing tip would be a single attached ligand, free to orient and rotate for unrestrained chemical activity during force spectroscopy. Using a molecular spacer with a length of a few nanometers between the cantilever tip and the attached ligand, the tip comes close to this ideal situation. Thanks to the softness and elasticity of the spacer it is possible to separate unspecific and specific interactions in ligand-receptor binding ${ }^{139}$, since specific interactions will make a soft probe bend much longer before rupture of the bond. Other advantages include the indeed free orientation of the ligand; the large surface of the ligand to scan for target molecules (clearly depending on the length of the spacer), which makes it easier to find a receptor in localisation studies but decreases resolution in force recognition mapping studies; and practically, a lower chance to damage the ligand upon hard contact with the surface ${ }^{145}$. It also removes the limitation that rigidly bound molecules may not allow important structural conformational changes in the receptor-ligand system. This is important since cell membrane receptors depend on conformational changes to get from an inactive to an active form.

The first spacer with two different functional groups on its ends (heterobifunctional) which was widely used for tip-PEG-protein linking is the PDP-PEG-NHS spacer. The functionalization of AFM tips with these spacers may be done by a standard three-step procedure ${ }^{63,145}$. The first step is to start with an amino-functionalised AFM tip where amino groups react with the $\mathrm{N}$ Hydroxysuccinimide (NHS) ester function, forming a stable amide bond. Usually out of all the PEG spacers attaching to the AFM tip, around 1-3 PEG spacers will be exactly in the reach under the apex of the AFM tip. Subsequently the pyridyldithiopropionyl (PDP) group of the spacer can react with a thiol group in a protein, resulting in a disulfide linkage between the PEG and a protein. Use of this direct method is only appropriate if the protein of interest contains a free thiol group. However, most extracellular proteins (e.g. antibodies) possess no free thiol group. A thiol group can then be introduced artificially using N-succinimidyl 3-(acetylthio)-propionate (SATP), a procedure not further elaborated. The result is a single molecule sensor with a receptor recognition probability up to $25 \%{ }^{146}$. Since the PEG spacers are covalently attached to the tip, the 
unbinding force of the biomolecule from the linker is higher than most ligand-receptor binding forces.

\subsubsection{Applications}

Single-molecule force spectroscopy (SMFS) by using AFM approach-retraction force curves is a powerful method for exploring the interaction forces of membrane molecules. Recording forcedistance curves by raster scanning an area, provides an easy but slow mapping method in order to get a force recognition image ${ }^{147}$; this method has been called force volume mapping or imaging, or spatially resolved SMFS. In order to image the recognition, the maximum negative deflection of the force-distance curve is depicted by the pixels. Force volume mapping can be used for assessing the distribution of receptors on the cell surface, although the lateral resolution of this simple method is $\operatorname{low}^{148}$. One of the first studies on ligand-receptor forces on living endothelial cells was performed on bovine aortic endothelial cells (KOM-1 cell line) by Almqvist et al. ${ }^{137}$ in 2004 . They mapped individual vascular endothelial growth factor receptors (VEGFR), and found that ligandenabled clustering on endothelial cells was less pronounced. Since then, several studies revealed properties of different receptors and cell membrane interactions ${ }^{142,149-152}$.

One of the latest progress in the application of studying membrane interactions by CFM is simultaneous topography and recognition imaging (TREC or dynamic recognition force mapping), which is done by dynamic AFM imaging ("tapping mode") with magnetically driven tips (MAC mode). In TREC, the interaction with the sample is recorded from the maxima and minima of the sinusoidal cantilever deflection period; the topography can be recorded by looking at the minima of the restrained oscillations, and the force recognition can be derived from the maxima of the restrained oscillations. This method requires a $\mathrm{Q}$ factor of the tip of around 1 in order to minimize the crossinterference between the minima and maxima of the tapping oscillation ${ }^{153}$. The TREC technique has a much higher lateral resolution down to $5 \mathrm{~nm}$ and can image much faster than before. This method was first applied on antibody tethered tips by Stroh et al. ${ }^{153}$, although Raab et al. ${ }^{154}$ already observed that antibody tethered tips showed more information than just topography in MAC mode. In 2007, Chtchelova et al. ${ }^{138}$ applied this technique for the first time on gently fixed and gelatin adhered cells (microvascular endothelial cells from mouse myocardium), to identify binding sites of vascular endothelial (VE)-cadherin. These receptors were found to be stable and did not exhibit clustering behaviour. In the same year, Lee et al. ${ }^{155}$ studied the binding of the same growth factor receptors on living and fixed human microvascular endothelial cells. They were able to image the receptors inhibition, visualised the non-uniform distribution, and determined the association and dissociation rate constants.

Manipulation or movement of biomolecules by functionalized AFM has so far only successfully been applied to DNA on model systems, where spacers between a DNA fragment and the AFM tip were used for higher recognition possibility ${ }^{156}$; manipulation on proteins has not been done so far (to the extent of our knowledge). The next step in AFM based recognition would be to present molecular stimuli on a tip to live cells and monitor the cellular response with advanced optical microscopy. Certainly the activity of the biomolecules incubated on the AFM tip will result in a cellular response in live cells, such that the AFM tip becomes an actuating indentation tool. This would open an interesting approach to manipulate and assess the actuation of proteins at the nano-scale and the subsequent cellular responses and protein pathways. In the final research chapter of this thesis we will elaborate on the methodological developments and the experiments performed of AFM based protein actuation. 


\subsection{References}

1. Kusumi, A. et al. Paradigm shift of the plasma membrane concept from the two-dimensional continuum fluid to the partitioned fluid: high-speed single-molecule tracking of membrane molecules. Annu. Rev. Biophys. Biomol. Struct. 34, 351-78 (2005).

2. Jacobson, K., Mouritsen, O. G. \& Anderson, R. G. W. Lipid rafts: at a crossroad between cell biology and physics. Nat. Cell Biol. 9, 7-14 (2007).

3. Shaw, A. S. Lipid rafts: now you see them, now you don't. Nat. Immunol. 7, 1139-42 (2006).

4. Engelman, D. M. Membranes are more mosaic than fluid. Nature 438, 578-80 (2005).

5. Simons, K. \& Gerl, M. J. Revitalizing membrane rafts: new tools and insights. Nat. Rev. Mol. Cell Biol. 11, 688-99 (2010).

6. Van Meer, G., Voelker, D. R. \& Feigenson, G. W. Membrane lipids: where they are and how they behave. Nat. Rev. Mol. Cell Biol. 9, 112-24 (2008).

7. Cebecauer, M., Spitaler, M., Sergé, A. \& Magee, A. I. Signalling complexes and clusters: functional advantages and methodological hurdles. J. Cell Sci. 123, 309-20 (2010).

8. Kholodenko, B. N., Hancock, J. F. \& Kolch, W. Signalling ballet in space and time. Nat. Rev. Mol. Cell Biol. 11, 414-426 (2010).

9. Citri, A. \& Yarden, Y. EGF-ERBB signalling: towards the systems level. Nat. Rev. Mol. Cell Biol. 7, 505-16 (2006).

10. Yarden, $Y$. \& Pines, G. The ERBB network: at last, cancer therapy meets systems biology. Nat. Rev. Cancer 12, 553-63 (2012).

11. Baselga, J. \& Swain, S. M. Novel anticancer targets: revisiting ERBB2 and discovering ERBB3. Nat. Rev. Cancer 9, 463-75 (2009).

12. Bader, A. N., Hofman, E. G., Voortman, J., en Henegouwen, P. M. P. V. B. \& Gerritsen, H. C Homo-FRET imaging enables quantification of protein cluster sizes with subcellular resolution. Biophys. J. 97, 2613-22 (2009).

13. Nagy, P. et al. Activation-dependent clustering of the erbB2 receptor tyrosine kinase detected by scanning near-field optical microscopy. J. Cell Sci. 112 (Pt 1, 1733-41 (1999).

14. Rong, G. \& Reinhard, B. M. Monitoring the size and lateral dynamics of ErbB1 enriched membrane domains through live cell plasmon coupling microscopy. PLoS One 7, e34175 (2012).

15. Ichinose, J., Murata, M., Yanagida, T. \& Sako, Y. EGF signalling amplification induced by dynamic clustering of EGFR. Biochem. Biophys. Res. Commun. 324, 1143-1149 (2004)

16. Kozer, N. et al. Exploring higher-order EGFR oligomerisation and phosphorylation--a combined experimental and theoretical approach. Mol. Biosyst. 9, 1849-63 (2013)

17. Hofman, E. G. Plasma membrane organization during EGFR signaling: a FRET-based analysis, PhD thesis, Utrecht University. (2008).

18. Hofman, E. G. et al. EGF induces coalescence of different lipid rafts. J. Cell Sci. 121, 2519-28 (2008).

19. Pryor, M. M. et al. Dynamic Transition States of ErbB1 Phosphorylation Predicted by Spatial Stochastic Modeling. Biophys. J. 105, 1533-43 (2013).

20. Radhakrishnan, K., Halász, Á., McCabe, M. M. Edwards, J. S. \& Wilson, B. S. Mathematical simulation of membrane protein clustering for efficient signal transduction. Ann. Biomed. Eng. 40, 2307-18 (2012).

21. Arkhipov, A. et al. Architecture and membrane interactions of the EGF receptor. Cell 152, 557-69 (2013).

22. Endres, N. F. et al. Conformational coupling across the plasma membrane in activation of the EGF receptor. Cell 152, 543-56 (2013).

23. Lemmon, M. A. \& Schlessinger, J. Cell signaling by receptor tyrosine kinases. Cell 141, 1117-34 (2010).

24. Betzig, E. et al. Imaging intracellular fluorescent proteins at nanometer resolution. Science 313, 16425 (2006).

25. RustMJ, B. Imaging by stochastic optical reconstruction microscopy (STORM). Nat. Methods 3, 793 (2006).

26. Hess, S. T., Girirajan, T. P. K. \& Mason, M. D. Ultrahigh resolution imaging by fluorescence photoactivation localization microscopy. Biophys. J. 91, 4258-72 (2006).

27. Peckys, D. B., Baudoin, J.-P., Eder, M., Werner, U. \& de Jonge, N. Epidermal growth factor receptor subunit locations determined in hydrated cells with environmental scanning electron microscopy. Sci. Rep. 3, 2626 (2013).

28. Low-Nam, S. T. et al. ErbB1 dimerization is promoted by domain co-confinement and stabilized by ligand binding. Nat. Struct. Mol. Biol. 18, 1244-1249 (2011).

29. Cutler, P. J. et al. Multi-color quantum dot tracking using a high-speed hyperspectral line-scanning microscope. PLoS One 8, e64320 (2013).

30. Chung, I. et al. Spatial control of EGF receptor activation by reversible dimerization on living cells. Nature 464, 783-7 (2010).

31. Gambin, Y. et al. Lateral mobility of proteins in liquid membranes revisited. Proc. Natl. Acad. Sci. U. S. A. 103, 2098-102 (2006).

32. De Keijzer, S. et al. A spatially restricted increase in receptor mobility is involved in directional sensing during Dictyostelium discoideum chemotaxis. J. Cell Sci. 121, 1750-7 (2008).

33. Kapanidis, A. N. \& Strick, T. Biology, one molecule at a time. Trends Biochem. Sci. 34, 234-43 (2009).

34. Simson, R., Sheets, E. D. \& Jacobson, K. Detection of temporary lateral confinement of membrane proteins using single-particle tracking analysis. Biophys. J. 69, 989-93 (1995).

35. Meilhac, N., Le Guyader, L., Salomé, L. \& Destainville, N. Detection of confinement and jumps in single-molecule membrane trajectories. Phys. Rev. E 73, 011915 (2006)

36. Michalet, X. Mean square displacement analysis of single-particle trajectories with localization error: Brownian motion in an isotropic medium. Phys. Rev. E 82, 041914 (2010)

37. Saxton, M. J. Lateral diffusion in an archipelago. Single-particle diffusion. Biophys. J. 64, 1766-80 (1993).

38. Elliott, L. C. C., Barhoum, M., Harris, J. M. \& Bohn, P. W. Trajectory analysis of single molecules exhibiting non-brownian motion. Phys. Chem. Chem. Phys. 13, 4326-34 (2011).

39. Berglund, A. Statistics of camera-based singleparticle tracking. Phys. Rev. E 82, 011917 (2010).

40. Montiel, D., Cang, H. \& Yang, H. Quantitative characterization of changes in dynamical behavior for 
single-particle tracking studies. J. Phys. Chem. B 110, 19763-70 (2006)

41. Persson, F., Lindén, M., Unoson, C. \& Elf, J. Extracting intracellular diffusive states and transition rates from single-molecule tracking data. Nat Methods 10, 265-9 (2013).

42. Harms, G. S., Cognet, L., Lommerse, P. H., Blab, G A. \& Schmidt, T. Autofluorescent proteins in singlemolecule research: applications to live cell imaging microscopy. Biophys. J. 80, 2396-408 (2001).

43. Xia, T., Li, N. \& Fang, X. Single-molecule fluorescence imaging in living cells. Annu. Rev. Phys. Chem. 64, 459-80 (2013).

44. Howarth, M. et al. Monovalent, reduced-size quantum dots for imaging receptors on living cells. Nat. Methods 5, 397-9 (2008).

45. Liu, H. Y. \& Gao, X. Engineering monovalent quantum dot-antibody bioconjugates with a hybrid gel system. Bioconjug. Chem. 22, 510-7 (2011).

46. Farlow, J. et al. Formation of targeted monovalent quantum dots by steric exclusion. Nat. Methods 10, 1203-5 (2013).

47. Wieser, S., Axmann, M. \& Schütz, G. J. Versatile analysis of single-molecule tracking data by comprehensive testing against Monte Carlo simulations. Biophys. J. 95, 5988-6001 (2008).

48. Keppler, A. et al. A general method for the covalent labeling of fusion proteins with small molecules in vivo. Nat. Biotechnol. 21, 86-9 (2003).

49. Keppler, A., Pick, H., Arrivoli, C., Vogel, H. \& Johnsson, K. Labeling of fusion proteins with synthetic fluorophores in live cells. Proc. Natl. Acad. Sci. U. S. A. 101, 9955-9 (2004).

50. Gautier, A. et al. An engineered protein tag for multiprotein labeling in living cells. Chem. Biol. 15, 128-36 (2008).

51. Sun, X. et al. Development of SNAP-tag fluorogenic probes for wash-free fluorescence imaging. Chembiochem 12, 2217-26 (2011).

52. Corrêa, I. R. et al. Substrates for improved live-cell fluorescence labeling of SNAP-tag. Curr. Pharm. Des. 19, 5414-20 (2013).

53. Pollack, V. A. et al. Inhibition of Epidermal Growth Factor Receptor-Associated Tyrosine Phosphorylation in Human Carcinomas with CP-358,774: Dynamics of Receptor Inhibition In Situ and Antitumor Effects in Athymic Mice. J. Pharmacol. Exp. Ther. 291, 739-748 (1999).

54. Rusnak, D. W. et al. The effects of the novel, reversible epidermal growth factor receptor/ErbB-2 tyrosine kinase inhibitor, GW2016, on the growth of human normal and tumor-derived cell lines in vitro and in vivo. Mol. Cancer Ther. 1, 85-94 (2001).

55. Li, S. et al. Structural basis for inhibition of the epidermal growth factor receptor by cetuximab. Cancer Cell 7, 301-11 (2005).

56. Ledford, H. Immunotherapy's cancer remit widens. Nature 497, 544 (2013).

57. Schaefer, G. et al. A two-in-one antibody against HER3 and EGFR has superior inhibitory activity compared with monospecific antibodies. Cancer Cell 20, 472-86 (2011)

58. Clayton, A. H. A., Tavarnesi, M. L. \& Johns, T. G. Unligated epidermal growth factor receptor forms higher order oligomers within microclusters on A431 cells that are sensitive to tyrosine kinase inhibitor binding. Biochemistry 46, 4589-97 (2007).

59. Gan, H. K. et al. The epidermal growth factor receptor (EGFR) tyrosine kinase inhibitor AG1478 increases the formation of inactive untethered EGFR dimers. Implications for combination therapy with monoclonal antibody 806. J. Biol. Chem. 282, 284050 (2007)

60. Clayton, A. H. a et al. Ligand-induced dimer-tetramer transition during the activation of the cell surface epidermal growth factor receptor-A multidimensional microscopy analysis. J. Biol. Chem. 280, 30392-9 (2005).

61. Verveer, P. J. Quantitative Imaging of Lateral ErbB1 Receptor Signal Propagation in the Plasma Membrane. Science 290, 1567-1570 (2000).

62. Ebner, A. et al. Functionalization of probe tips and supports for single-molecule recognition force microscopy. Top. Curr. Chem. 285, 29-76 (2008)

63. Ebner, A. et al. A new, simple method for linking of antibodies to atomic force microscopy tips. Bioconjug. Chem. 18, 1176-1184 (2007).

64. Puntheeranurak, T., Wildling, L., Gruber, H. J., Kinne, R. K. H. \& Hinterdorfer, P. Ligands on the string: single-molecule AFM studies on the interaction of antibodies and substrates with the Na+-glucose cotransporter SGLT1 in living cells. J. Cell Sci. 119, 2960-7 (2006)

65. Ebner, A. et al. in Handbook of Single-Molecule Biophysics 407-447 (Springer, 2009). doi:10.1007/978-0-387-76497-9 15

66. Lamontagne, C.-A., Cuerrier, C. M. \& Grandbois, M. AFM as a tool to probe and manipulate cellular processes. Pflugers Arch. 456, 61-70 (2008).

67. Posch, S. et al. Activation induced morphological changes and integrin $\alpha \mathrm{llb} \beta 3$ activity of living platelets. Methods 60, 179-85 (2013)

68. Yarden, Y. \& Sliwkowski, M. X. Untangling the ErbB signalling network. Nat. Rev. Mol. Cell Biol. 2, 127-37 (2001).

69. Lemmon, M. a. Ligand-induced ErbB receptor dimerization. Exp. Cell Res. 315, 638-48 (2009).

70. Robert, P., Benoliel, A.-M., Pierres, A. \& Bongrand $P$. What is the biological relevance of the specific bond properties revealed by single-molecule studies? J. Mol. Recognit. 20, 432-47 (2007).

71. Alberts, B. et al. Molecular Biology of the Cell. (Garland Publishing Inc,US, 2008).

72. Stortelers, C. The role of EGF-like growth factors in selective ErbB receptor dimerisation, PhD thesis, Radboud Universiteit Nijmegen. (2003).

73. Landgraf, R. HER2 therapy. HER2 (ERBB2): functional diversity from structurally conserved building blocks. Breast Cancer Res. 9, 202 (2007).

74. Graus-Porta, D., Beerli, R. R., Daly, J. M. \& Hynes, N. E. ErbB-2, the preferred heterodimerization partner of all ErbB receptors, is a mediator of lateral signaling. EMBO J. 16, 1647-55 (1997)

75. Moasser, M. M. The oncogene HER2: its signaling and transforming functions and its role in human cancer pathogenesis. Oncogene 26, 6469-87 (2007).

76. Slamon, D. et al. Human breast cancer: correlation of relapse and survival with amplification of the HER2/neu oncogene. Science 235, 177-182 (1987)

77. Shi, F., Telesco, S. E., Liu, Y., Radhakrishnan, R. \& Lemmon, M. A. ErbB3/HER3 intracellular domain is competent to bind ATP and catalyze autophosphorylation. Proc. Natl. Acad. Sci. U. S. A 107, 7692-7 (2010).

78. Taylor, S. S. \& Kornev, A. P. Yet another "active" pseudokinase, Erb3. Proc. Natl. Acad. Sci. U. S. A 107, 8047-8 (2010).

79. Jiang, N., Saba, N. F. \& Chen, Z. G. Advances in Targeting HER3 as an Anticancer Therapy. Chemother. Res. Pract. 2012, 817304 (2012). 
80. Ferguson, K. M. Structure-based view of epidermal growth factor receptor regulation. Annu. Rev. Biophys. 37, 353-73 (2008).

81. Ferguson, K. M., Darling, P. J., Mohan, M. J., Macatee, T. L. \& Lemmon, M. a. Extracellular domains drive homo- but not hetero-dimerization of erbB receptors. EMBO J. 19, 4632-43 (2000).

82. Martin-Fernandez, M., Clarke, D. T., Tobin, M. J., Jones, S. V \& Jones, G. R. Preformed oligomeric epidermal growth factor receptors undergo an ectodomain structure change during signaling. Biophys. J. 82, 2415-27 (2002).

83. Nagy, P. Lipid rafts and the local density of ErbB proteins influence the biological role of homo- and heteroassociations of ErbB2. J. Cell Sci. 115, 42514262 (2002).

84. Moriki, T., Maruyama, H. \& Maruyama, I. N. Activation of preformed EGF receptor dimers by ligand-induced rotation of the transmembrane domain. J. Mol. Biol. 311, 1011-26 (2001)

85. Gadella, T. W. \& Jovin, T. M. Oligomerization of epidermal growth factor receptors on A431 cells studied by time-resolved fluorescence imaging microscopy. A stereochemical model for tyrosine kinase receptor activation. J. Cell Biol. 129, 1543-58 (1995)

86. Nagy, P., Claus, J., Jovin, T. M. \& Arndt-Jovin, D. J. Distribution of resting and ligand-bound ErbB1 and ErbB2 receptor tyrosine kinases in living cells using number and brightness analysis. Proc. Natl. Acad. Sci. U. S. A. 107, 16524-9 (2010).

87. Keating, E., Nohe, A. \& Petersen, N. O. Studies of distribution, location and dynamic properties of EGFR on the cell surface measured by image correlation spectroscopy. Eur. Biophys. J. 37, 469-81 (2008)

88. Teramura, $Y$. et al. Single-molecule analysis of epidermal growth factor binding on the surface of living cells. EMBO J. 25, 4215-22 (2006).

89. Sorkin, a. Internalization of the epidermal growth factor receptor: role in signalling. Biochem. Soc. Trans. 29, 480-4 (2001).

90. Sorkin, A. \& Waters, C. M. Endocytosis of growth factor receptors. Bioessays 15, 375-82 (1993).

91. Lidke, D. S. et al. Quantum dot ligands provide new insights into erbB/HER receptor-mediated signal transduction. Nat. Biotechnol. 22, 198-203 (2004).

92. Waterman, H. \& Yarden, Y. Molecular mechanisms underlying endocytosis and sorting of ErbB receptor tyrosine kinases. FEBS Lett. 490, 142-52 (2001).

93. Sorkin, A. \& Goh, L. K. Endocytosis and intracellular trafficking of ErbBs. Exp. Cell Res. 315, 683-696 (2009).

94. Orr, G. et al. Cholesterol dictates the freedom of EGF receptors and HER2 in the plane of the membrane. Biophys. J. 89, 1362-73 (2005).

95. Van Zanten, T. S. et al. Hotspots of GPI-anchored proteins and integrin nanoclusters function as nucleation sites for cell adhesion. Proc. Natl. Acad. Sci. U. S. A. 106, 18557-62 (2009).

96. Plowman, S. J., Muncke, C., Parton, R. G. \& Hancock, J. F. H-ras, K-ras, and inner plasma membrane raft proteins operate in nanoclusters with differential dependence on the actin cytoskeleton. Proc. Natl. Acad. Sci. U. S. A. 102, 15500-5 (2005)

97. Cambi, A. \& Lidke, D. S. Nanoscale membrane organization: where biochemistry meets advanced microscopy. ACS Chem. Biol. 7, 139-49 (2012).

98. Kwik, J. et al. Membrane cholesterol, lateral mobility, and the phosphatidylinositol 4,5-bisphosphatedependent organization of cell actin. Proc. Natl. Acad. Sci. U. S. A. 100, 13964-9 (2003).
99. Hofman, E. G. et al. Ligand-induced epidermal growth factor receptor (EGFR) oligomerization is kinase-dependent and enhances internalization. $J$. Biol. Chem. 285, 39481-39489 (2010).

100. Wang, Q., Zhu, F. \& Wang, Z. Identification of EGF receptor C-terminal sequences 1005-1017 and dileucine motif 1010LL1011 as essential in EGF receptor endocytosis. Exp. Cell Res. 313, 3349-63 (2007).

101. Saffarian, S., Li, Y., Elson, E. L. \& Pike, L. J. Oligomerization of the EGF receptor investigated by live cell fluorescence intensity distribution analysis. Biophys. J. 93, 1021-31 (2007)

102. Kusumi, a, Sako, Y. \& Yamamoto, M. Confined lateral diffusion of membrane receptors as studied by single particle tracking (nanovid microscopy). Effects of calcium-induced differentiation in cultured epithelial cells. Biophys. J. 65, 2021-40 (1993).

103. Holtzer, L. \& Schmidt, T. in Single Particle Tracking and Single Molecule Energy Transfer (Bräuchle, C., Lamb, D. C. \& Michaelis, J.) (WILEY-VCH Verlag $\mathrm{GmbH}, 2010$ )

104. Sergé, A., Bertaux, N., Rigneault, H. \& Marguet, D. Dynamic multiple-target tracing to probe spatiotemporal cartography of cell membranes. Nat. Methods 5, 687-94 (2008).

105. Schütz, G. J., Schindler, H. \& Schmidt, T. Singlemolecule microscopy on model membranes reveals anomalous diffusion. Biophys. J. 73, 1073-80 (1997)

106. Brameshuber, M. \& Schütz, G. J. in Springer Series on Fluorescence 1-37 (Springer Berlin Heidelberg, 2012). doi:10.1007/4243_2011_38

107. Dietrich, C., Yang, B., Fujiwara, T., Kusumi, A. \& Jacobson, K. Relationship of lipid rafts to transient confinement zones detected by single particle tracking. Biophys. J. 82, 274-84 (2002).

108. Harding, A. S. \& Hancock, J. F. Using plasma membrane nanoclusters to build better signaling circuits. Trends Cell Biol. 18, 364-371 (2008).

109. Hsieh, M., Yang, S., Raymond-Stinz, M. A., Edwards, J. S. \& Wilson, B. S. Spatio-temporal modeling of signaling protein recruitment to EGFR. BMC Syst. Biol. 4, 57 (2010).

110. Harding, A. Ras nanoclusters: combining digital and analog signaling. Cell Cycle 127-134 (2008).

111. Wieser, S. \& Schütz, G. J. Tracking single molecules in the live cell plasma membrane-Do's and Don't's. Methods 46, 131-40 (2008).

112. Jaqaman, $\mathrm{K}$. et al. Robust single-particle tracking in live-cell time-lapse sequences. Nat. Methods 5, 695$702(2008)$

113. Smith, C. S., Joseph, N., Rieger, B. \& Lidke, K. A. Fast, single-molecule localization that achieves theoretically minimum uncertainty. Nat. Methods 7 , 373-5 (2010).

114. Giepmans, B. N. G., Adams, S. R., Ellisman, M. H. \& Tsien, R. Y. The fluorescent toolbox for assessing protein location and function. Science 312, 217-24 (2006).

115. Lidke, D. S., Low-Nam, S. T., Cutler, P. J. \& Lidke, K. A. Determining FcERI diffusional dynamics via single quantum dot tracking. Methods Mol. Biol. 748, 121-32 (2011).

116. Digman, M. a, Dalal, R., Horwitz, A. F. \& Gratton, E. Mapping the number of molecules and brightness in the laser scanning microscope. Biophys. J. 94, 232032 (2008).

117. Petersen, N. O., Höddelius, P. L., Wiseman, P. W., Seger, O. \& Magnusson, K. E. Quantitation of membrane receptor distributions by image correlation 
spectroscopy: concept and application. Biophys. J. 65, 1135-46 (1993).

118. Wiseman, P. W. \& Petersen, N. O. Image correlation spectroscopy. II. Optimization for ultrasensitive detection of preexisting platelet-derived growth factorbeta receptor oligomers on intact cells. Biophys. J. 76, 963-77 (1999).

119. Jares-Erijman, E. A. \& Jovin, T. M. FRET imaging. Nat. Biotechnol. 21, 1387-95 (2003).

120. Lakowicz, J. R. Principles of Fluorescence Spectroscopy. (Springer US, 2006). doi:10.1007/9780-387-46312-4

121. Miyawaki, A. Proteins on the move: insights gained from fluorescent protein technologies. Nat. Rev. Mol. Cell Biol. 12, 656-68 (2011).

122. Davidson, M. W. \& Campbell, R. E. Engineered fluorescent proteins: innovations and applications. Nat. Methods 6, 713-717 (2009)

123. Filonov, G. S. et al. Bright and stable near-infrared fluorescent protein for in vivo imaging. Nat. Biotechnol. 29, 757-61 (2011).

124. Azhdarinia, A., Ghosh, P., Ghosh, S., Wilganowski N. \& Sevick-Muraca, E. M. Dual-labeling strategies for nuclear and fluorescence molecular imaging: a review and analysis. Mol. Imaging Biol. 14, 261-76 (2012).

125. Sampath, L. et al. Dual-labeled trastuzumab-based imaging agent for the detection of human epidermal growth factor receptor 2 overexpression in breast cancer. J. Nucl. Med. 48, 1501-10 (2007).

126. Orrit, M. Chemical and physical aspects of charge transfer in the fluorescence intermittency of single molecules and quantum dots. Photochem. Photobiol. Sci. 9, 637-42 (2010).

127. Pinaud, F., Clarke, S., Sittner, A. \& Dahan, M. Probing cellular events, one quantum dot at a time. Nat. Methods 7, 275-85 (2010).

128. Semrau, S. \& Schmidt, T. Particle image correlation spectroscopy (PICS): retrieving nanometer-scale correlations from high-density single-molecule position data. Biophys. J. 92, 613-21 (2007).

129. Smith, A. \& Nie, S. Minimizing the hydrodynamic size of quantum dots with multifunctional multidentate polymer ligands. J. Am. Chem. Soc. 130, 1127811279 (2008)

130. Bustamante, C., Macosko, J. C. \& Wuite, G. J. Grabbing the cat by the tail: manipulating molecules one by one. Nat. Rev. Mol. Cell Biol. 1, 130-6 (2000).

131. Ashkin, A. Acceleration and Trapping of Particles by Radiation Pressure. Phys. Rev. Lett. 24, 156-159 (1970).

132. Huisstede, J. Scanning probe optical tweezers, PhD thesis, University of Twente. doc.utwente.nl (2006).

133. Walter, N. G., Huang, C.-Y., Manzo, A. J. \& Sobhy, M. A. Do-it-yourself guide: how to use the modern single-molecule toolkit. Nat. Methods 5, 475-89 (2008).

134. Frétigny, C. in Nanoscience 91 - 119 (Springer Berlin Heidelberg, 2007). doi:10.1007/978-3-54028617-2

135. Alessandrini, A. \& Facci, P. AFM: a versatile tool in biophysics. Meas. Sci. Technol. 16, R65-R92 (2005).

136. Selvin, P. R. \& Ha, T. Single Molecule Techniques: A Laboratory Manual. 507 (Cold Spring Harbor Laboratory Press,U.S., 2007).

137. Almqvist, N. et al. Elasticity and adhesion force mapping reveals real-time clustering of growth factor receptors and associated changes in local cellular rheological properties. Biophys. J. 86, 1753-62 (2004).
138. Chtcheglova, L. A., Waschke, J., Wildling, L. Drenckhahn, D. \& Hinterdorfer, P. Nano-scale dynamic recognition imaging on vascular endothelial cells. Biophys. J. 93, L11-3 (2007).

139. Hinterdorfer, P., Baumgartner, W., Gruber, H. J., Schilcher, K. \& Schindler, H. Detection and localization of individual antibody-antigen recognition events by atomic force microscopy. Proc. Natl. Acad. Sci. U. S. A. 93, 3477-81 (1996).

140. Lee, G. U., Kidwell, D. A. \& Colton, R. J. Sensing Discrete Streptavidin-Biotin Interactions with Atomic Force Microscopy. Langmuir 10, 354-357 (1994).

141. Hinterdorfer, P. \& Dufrêne, Y. F. Detection and localization of single molecular recognition events using atomic force microscopy. Nat. Methods 3, 347355 (2006).

142. Müller, D. J., Krieg, M., Alsteens, D. \& Dufrêne, Y. F. New frontiers in atomic force microscopy: analyzing interactions from single-molecules to cells. Curr. Opin. Biotechnol. 20, 4-13 (2009).

143. Zlatanova, J. Single molecule force spectroscopy in biology using the atomic force microscope. Prog. Biophys. Mol. Biol. 74, 37-61 (2000).

144. Moy, V., Florin, E. \& Gaub, H. Intermolecular forces and energies between ligands and receptors. Science 266, 257-259 (1994).

145. Riener, C. K. et al. Heterobifunctional crosslinkers for tethering single ligand molecules to scanning probes. Anal. Chim. Acta 497, 101-114 (2003).

146. Kamruzzahan, A. S. M. et al. Antibody linking to atomic force microscope tips via disulfide bond formation. Bioconjug. Chem. 17, 1473-81 (2006).

147. Willemsen, O. H. et al. Simultaneous height and adhesion imaging of antibody-antigen interactions by atomic force microscopy. Biophys. J. 75, 2220-8 (1998).

148. Klein, D. C. G. Feeling sugar-protein interactions using carbon nanotubes, $\mathrm{PhD}$ thesis, University of Leiden. (2004).

149. Müller, D. \& Dufrêne, Y. Atomic force microscopy as a multifunctional molecular toolbox in nanobiotechnology. Nat. Nanotechnol. 3, 261-269 (2008).

150. Müller, D. J. et al. Single-molecule studies of membrane proteins. Curr. Opin. Struct. Biol. 16, 48995 (2006)

151. Chtcheglova, L. A., Wildling, L., Waschke, J., Drenckhahn, D. \& Hinterdorfer, P. AFM functional imaging on vascular endothelial cells. J. Mol. Recognit. 23, 589-96 (2010).

152. Duman, M. et al. Nanomapping of CD1d-glycolipid complexes on THP1 cells by using simultaneous topography and recognition imaging. J. Mol. Recognit. 26, 408-14 (2013).

153. Stroh, C. M. et al. Simultaneous topography and recognition imaging using force microscopy. Biophys. J. 87, 1981-90 (2004).

154. Raab, A. et al. Antibody recognition imaging by force microscopy. Nat. Biotechnol. 17, 901-5 (1999).

155. Lee, S., Mandic, J. \& Van Vliet, K. J. Chemomechanical mapping of ligand-receptor binding kinetics on cells. Proc. Natl. Acad. Sci. U. S. A. 104, 9609-14 (2007).

156. Kufer, S. K., Puchner, E. M., Gumpp, H., Gaub, H. E. \& Liedl, T. Single-molecule cut-and-paste surface assembly. Science 319, 594-6 (2008). 



\section{Chapter 2}

Single-molecule tracking microscopy 


\subsection{Introduction}

We have built a custom fluorescence microscope with total internal reflection fluorescence (TIRF) illumination, to be able to detect single molecules in a very short time (typically $<20 \mathrm{~ms}$ to prevent motion blur). To obtain enough photons from one fluorescent molecule within this time requires high illumination intensities within a field of view of at least one cell. Such microscopes are not readily commercially available (yet), which motivates research groups in the single-molecule tracking field to use custom-built microscopes ${ }^{1-5}$. Furthermore, these custom-built microscopes use a minimal number of components to yield very high detection efficiencies, whereas commercial microscopes often make concessions in this to facilitate easier plugin of modules or modalities. Since fluorescent dyes can only emit a limited number of photons, we want to collect as many of these emitted photons as possible without losses. In principal, a commercially available STORM microscope (using TIRF) would be suited to perform single-molecule tracking as well. However, the price of components necessary for an optimized custom-built microscope with high illumination powers comes at a fraction of the cost of a commercial STORM microscope with moderate illumination power.

In this chapter we describe in detail the requirements and implementation of a single molecule sensitive fluorescence microscope to track single proteins in live cells. We provide a thorough characterization of our custom-built microscope, and show that the setup can detect single fluorescent molecules with a localization accuracy of $20-40 \mathrm{~nm}$ within $2-20 \mathrm{~ms}$. We show that the use of solid-state lasers is a cost-effective alternative to the widely used gas lasers for this application, without compromising image quality. We describe the use of a rotating diffuser to provide high-intensity incoherent excitation light which can be coupled into the microscope without optical fibres using a Kohler illumination scheme. To construct protein trajectories from the recorded image series, we used one of the latest tracking software available, which is currently still in development. Furthermore, we describe the analysis methods currently used to characterize the motion of proteins in live cells from the trajectories obtained after tracking.

\subsection{Design and realization of a single-molecule tracking microscope}

An objective based total internal reflection fluorescence (TIRF) microscope was built upon a standard inverted fluorescence microscope (Zeiss 135TV) to be able to quickly change samples and easily switch to a brightfield microscope mode to focus on the sample (Fig. 2.1). We removed the tube lens of the microscope body to increase the detection efficiency, and to create space to place filters. The illumination light was provided by low-cost solid-state lasers, and was coupled into the microscope by Köhler illumination with a rotating diffuser in the light source plane. The microscope was equipped with an Olympus PlanApo 100x/1,45 Oil TIRF objective. Samples were heated to $35-37^{\circ} \mathrm{C}$ on the microscope with a stage heating plate and an objective ring heater. The images were acquired using two Andor iXon EM+ DU-897 back illuminated EMCCD cameras. We describe the components used and the illumination scheme in more detail below.

\subsubsection{Camera}

\subsubsection{Requirements}

To image the few photons from a single molecule, a sensitive camera with low internal noise sources is necessary to discriminate the signal from the sample background with a high enough frame rate to allow tracking of a fluorescent molecule. Two common sources of noise in cameras are read-out noise (originating from the charge-to-voltage converter), and dark current noise(induced by thermal electronic fluctuations). Different types of modern scientific cameras (e.g. intensified CCD (ICCD), electron multiplying CCD (emCCD), scientific CMOS (sCMOS) or 

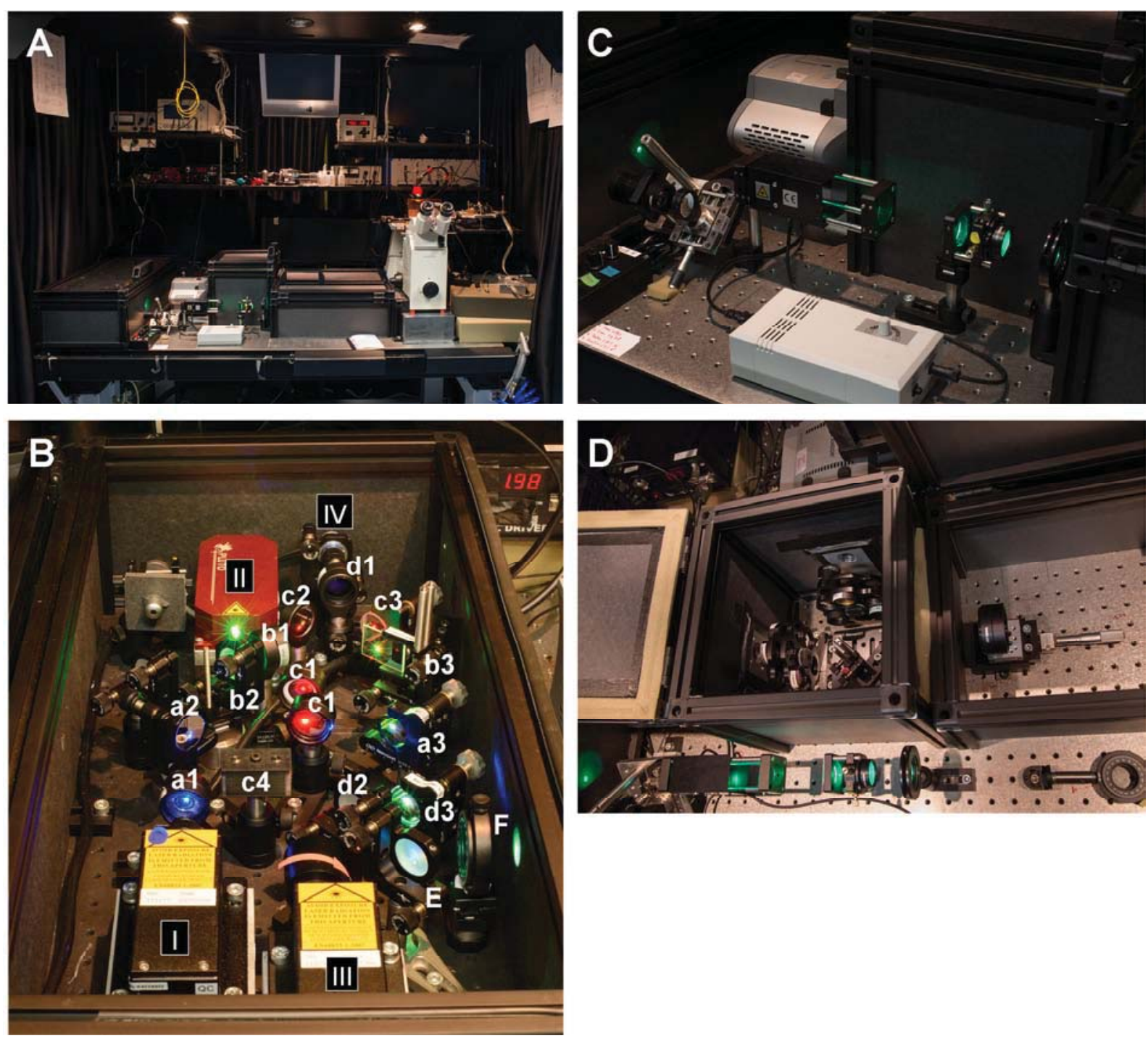

FIGURE 2.1 Photographs of the single-molecule tracking microscope. (A) Overview of the microscope setup showing the implementation of a custom excitation and detection path to a Zeiss 135TV microscope body. (B) The lasers and optical scheme to combine the laser light to provide intense illumination light of different wavelengths. Laser I emits light at $473 \mathrm{~nm}$, laser II at $532 \mathrm{~nm}$, laser III at $637 \mathrm{~nm}$, and laserpointer IV at 405 $\mathrm{nm}$. The laser light is passed through bandpass filters (1, a-d for the corresponding lasers), to obtain single wavelength light from each laser. The light is aligned using reflective mirrors (2) and combined using longpass dichroic mirrors (3). The reflected red light from laser $C$ is dumped on a light sink $c 4$. Mirror $E$ reflects the combined excitation light towards the illumination scheme. A quarter wave plate $F$ is used to obtain circularly polarized light. (C) Implementation of a rotating diffuser (middle) and the flip-in TIRF module (left). One lens on the TIRF module can be laterally shifted to move the focal spot on the diffuser and thereby change the (TIRF) angle of incidence at the interface of the sample. (D) The detection scheme after the dichroic mirror that separates the excitation from the emission light. A relocated imaging lens focusses the image plane onto the CCD chips of the two cameras. An image quality dichroic mirror, mounted on a kinematic base for quick release, separates the emission light for simultaneous two-colour imaging. Several emission filters can be quickly selected from the cage filter wheel before each camera. 
cooled CCD) all have adequate ways to circumvent these noise sources ${ }^{6}$, and the best choice of the type of camera therefore depends mostly on the required frame rate.

To estimate the optimal frame rate, we need to determine the minimal frame rate required to track receptor molecules, i.e. we need all molecules observed in a frame to be identifiable without ambiguity in the next frame. For this, the number of molecules in the recording should be low enough such that the distance of a molecule travelled between frames is significantly smaller than the average distance between nearest neighbours. The exact calculation for the needed frame rate is quite complex; therefore we provide a rough estimate. Let us assume that a molecule can still be tracked when the maximum distance travelled between frames is 4 pixels on the camera. This value seems reasonable when we regard a field of view of 400x400 pixels with $120 \mathrm{~nm} / \mathrm{pixel}$, and a total number of 200 molecules; a maximum search distance of 4-5 pixels is also used in earlier studies $^{3,7,8}$. Since $99 \%$ of the step sizes are smaller than a certain value ${ }^{7}$ given by:

$$
R_{\text {max }}=2.55 \cdot \sqrt{4 D \tau_{\text {lag }}}
$$

we find that to obtain $R_{\max }<4$ pixels $=475 \mathrm{~nm}$ for molecules with $D=0.15 \mu \mathrm{m}^{2} / \mathrm{s}$, we need $\tau_{\text {lag }}<$ $58 \mathrm{~ms}$. The mean displacement is in this particular situation $\sqrt{4 D \tau_{\text {lag }}}=190 \mathrm{~nm}$ (1.6 pixels). The value for $\tau_{\text {lag }}$ means that a minimum frame rate of $17 \mathrm{fps}$ is needed to allow tracking of receptors of the ErbB family studied in this thesis. The number of photons that can be obtained from one molecule within $58 \mathrm{~ms}$ is also sufficient to localize this molecule (section 2.2.2.1).

sCMOS cameras tend to have the highest possible read-out speeds and a large field of view. Yet emCCDs have a better signal-to-noise ratio (SNR) for a low number of photons per pixel. The breakeven point between emCCDs and sCMOS cameras is currently around 5 photons per pixel ${ }^{9}$. This means that emCCDs will obtain the best localization precision for low-intensity fluorophores, such as single organic dyes.

\subsubsection{Realization}

Since we already possessed two Andor iXon EM+ DU-897 cameras, which are very well suited for low intensity imaging ${ }^{6,10}$, we chose to utilize these during the measurements described in this thesis. The dark current of this camera is documented to be 0.001 electron per pixel per second ${ }^{11}$. The maximum read-out rate of this camera is $10 \mathrm{MHz}$ with 14 bit digitization, allowing up to 35 fps for the complete field of view of 512x512 pixels. With an exposure time of $9 \mathrm{~ms}$, the maximum frame rate reduces to $26 \mathrm{fps}$, which is well above the required frame rate ( $17 \mathrm{fps})$. At the maximum read-out rate and under electron multiplying conditions, the effective system readout noise is below one electron per pixel. The quantum yield of the camera is above $90 \%$ for wavelengths between $475 \mathrm{~nm}$ and $700 \mathrm{~nm}$. However, the amplification process in emCCDs results in an increase in shot noise to the signal by the excess noise factor (approximately $\sqrt{2}$ ). Effectively this has a similar effect as having a camera with half the quantum efficiency ${ }^{6}$. The size of the pixels is $16 \times 16 \mu \mathrm{m}$, such that a magnification of $150 \mathrm{x}$ would result in $107 \mathrm{~nm} /$ pixel. Furthermore, the camera has TTL input and output ports to allow synchronized recording on two cameras and to synchronise the illumination to happen only during camera acquisition periods.

\subsubsection{Light source}

\subsubsection{Requirements}

To obtain enough photons from single molecules within the milliseconds exposure time typically available per frame to obtain a recording without much motion blur, relatively high illumination intensities are needed. Enough photons are needed since the localization accuracy is 
fundamentally limited (in the condition without background signals) by the number of photons according to:

$$
\sigma_{x y}=\sigma_{G} / \sqrt{N}
$$

where $\sigma_{G}$ is the Gaussian radius of the diffraction limited spot, and $N$ is the number of photons detected $^{12}$. The minimum illumination intensity required therefore depends on the fluorophore used and the diffusion speed of the molecule it is attached to. Furthermore, we need light sources that have low spatial divergence to efficiently couple the light into the microscope, a fast modulation capability to effectively reduce the bleaching of the fluorophore outside the camera acquisition period, and the availability of multiple excitation wavelengths to perform multiplecolour experiments. Since we are not required to excite particular genetically encoded fluorophores (such as cyan, green or yellow fluorescent protein, i.e. CFP, GFP or YFP), we are not dependent on standard gas laser line wavelengths.

The need for a light source with high intensity is explained by the following example. The average distance travelled by a molecule with diffusion coefficient $D_{\max }=0.15 \mu \mathrm{m}^{2} / \mathrm{s}$ within $6 \mathrm{~ms}$ is $60 \mathrm{~nm}$ (0.5 pixel), which convolves with the Gaussian radius of the diffraction limited spot (typically $100-120 \mathrm{~nm}$, or 1 pixel). Longer acquisition times (>5ms) will result in a significant distortion ( $>20 \%$ radius increase) of the diffraction limited spot. Therefore when making recordings of this molecule, sufficient photons must be collected from the molecule within this acquisition time. At this acquisition time, it is found using Eq. 2 that we need at least 36 photons to achieve a localization accuracy of $20 \mathrm{~nm}$ from a diffraction limited spot with a convolved Gaussian radius of $120 \mathrm{~nm}$. The required localization accuracy is typically unknown beforehand; when a lower value is achieved, fewer trajectories for a certain condition will be necessary for analysis to obtain robust results. In practice, to clearly observe a single fluorescent molecule at this localization accuracy in the cellular fluorescence background requires even more photons. We estimate that a fluorescent signal with at least 100 photons is required in a wide field setup to yield this localization accuracy. When utilizing TIRF illumination, this number will be lower due to the decreased level of fluorescent background. We expect that the microscope detects $8 \%$ of the emitted photons, since an 1.45 NA objective with immersion oil with $n=1.52$ acquires $16 \%$ of all the light emitted by a monopole, and the estimated detection efficiency is $50 \%$. Under these assumptions, the minimal needed photon emission rate $I_{e m}$ is 250,000 photons per second. Taking a typical cross section $\sigma$ of a fluorophore to be $2\left(\AA^{2}\right)$ and a fluorophore quantum efficiency $Q$ of 0.8 , we obtain using Eq. 3 a required excitation intensity $\Phi_{e x}$ of $1.5 \times 10^{21}$ photons per $\mathrm{cm}^{2}$ per second.

$$
I_{e m}=\Phi_{e x} \cdot Q \cdot \sigma
$$

Given a photon at $550 \mathrm{~nm}$ we get using $E=h c / \lambda$, with $h=6.626 \times 10^{-34}(\mathrm{~J} \cdot \mathrm{s})$ and $c=2.998 \times 10^{8}$ $(\mathrm{m} / \mathrm{s}), E_{\text {photon }}=3.62 \times 10^{-19}(\mathrm{~J})$. Hence the excitation intensity corresponds to $0.57 \mathrm{~kW} / \mathrm{cm}^{2}$. Illumination using the quite homogeneous center part of a Gaussian shaped image (as for lasers) requires at least 4 times more power due to the non-used edges of the image beam. For a 40x40 $\mu \mathrm{m}$ area, and a well-designed $50 \%$ efficient beampath for excitation filters and lenses (the illumination scheme), we then easily find that a laser of at least $75 \mathrm{~mW}$ is required. When the excitation time has to be lower than $5 \mathrm{~ms}$ to reduce the blurring effect by shorter (stroboscopic) illumination, the laser power required scales up inversely. Most single-molecule microscopes are designed with an excitation intensity in the range $0.1-5 \mathrm{~kW} / \mathrm{cm}^{2}$. Certainly the saturation intensity of the fluorophore should not be exceeded, since the background noise will then start to have a negative effect on the signal-to-noise. The intensity of fluorescent proteins start to saturate ${ }^{13}$ at 10 $\mathrm{kW} / \mathrm{cm}^{2}$, and the saturation intensity for organic dyes is even higher ${ }^{14}$. 
Lower-cost solid-state lasers provide easier installation and operation compared to using gas lasers or highly stabilized solid-state lasers, since the latter cannot be modulated by the laser controller itself, and require an acousto-optical tunable filter (AOTF). Such filters rarely achieve a full deflection to suppress complete transmission, and are a more costly solution. Furthermore the high beam quality or high frequency power stability of higher-cost lasers are not often critical requirements for single-molecule microscopy illumination.

\subsubsection{Realization}

For the excitation we used a $532 \mathrm{~nm} 400 \mathrm{~mW}$ solid state laser from Pegasus Shanghai Optical Systems (Pegasus Lasersysteme, Wallenhorst, Germany), and a 637nm 300mW diode laser from Changchun New Industries Optoelectronics (Laser 2000, Vinkeveen, The Netherlands). These lasers are a cost-effective solution to the requirements set above, and provide well-separated excitation wavelengths for two organic dyes with distinct excitation and emission spectra to allow simultaneous imaging. Furthermore, these lasers have very little divergence $(<1.0 \mathrm{mrad})$, have an RMS power stability $<3 \%$, and come with a TTL modulation up to $10 \mathrm{kHz}$. These lower-cost solid-state lasers have larger emission wavelength bandwidth. Wavelengths much higher than the centre wavelength, which pass the microscope's emission filters, must be efficiently filtered. The related low coherence length of the laser is typically an advantage for microscopy illumination, since the use of light with a high coherence length result in unwanted interference effects in the illumination area.

The infrared and off-centre wavelengths produced by the lasers were not sufficiently suppressed by the internal filters, and therefore bandpass filters were inserted in the excitation path after the lasers to suppress undesirable wavelengths in the excitation light. The green laser light was filtered by an FF01-543/22 (SemRock, Rochester, NY) filter and the red laser light was filtered by a D644/10m (Chroma, Olching, Germany) bandpass filter. The two laser beams were combined with a 610DRXXR (unknown manufacturer) longpass mirror.

An achromatic quarter wave plate (VM-TIM Optomechanische Werke, Jena, Germany) was inserted in the beam path to obtain circularly polarized excitation light. This reduces signal intensity differences otherwise observed due to the dipole orientation of a fluorophore. For the $532 \mathrm{~nm}$ laser there was less than $1 \%$ power difference in the elliptical polarization axes, for the 637 $\mathrm{nm}$ laser the elliptical polarization axes had a power ratio of $0.92: 1$.

The chosen excitation filter, dichroic mirror and wave plate must be suitable for the high light intensity coming from the lasers (documented diameter of laser beam $1 \mathrm{~mm}$ ). The allowed CW light intensity is usually not specified for filters. We did not observe burning effects on the filters with the laser at maximum power, even with continuous illumination for more than 10 minutes.

\subsubsection{Illumination scheme}

For laser based widefield illumination, the light is usually focused on the back focal plane of the objective, which means that the light is again perpendicular after the objective and through the sample. In that case, only the central part of the Gaussian shaped laser beam is used for illumination of the sample because it has a homogeneous intensity; as a consequence, a lot of the incident light is not used. Furthermore, the complete sample is illuminated by perpendicular light rays, which might result in background fluorescence. Clearly, this is not the most efficient scheme for illumination, and we chose to make use of a rotating diffuser in a transmission Köhler illumination scheme (Fig. 2.2) to produce a homogenous and focused illumination in the objective's focal plane, with an adjustable field of view. A small angle diffuser (SUSS MicroOptics, Hauterive, Switzerland), consisting of randomly distributed microlens units, was used. In a Köhler scheme, the amount of background fluorescence will be lower compared to perpendicular illumination, which is especially important in imaging the relatively low signals of single molecules. 
Another issue is the high coherence length of laser light, which can result in interference between reflections from the various glass interfaces: all the lenses, the objective, the coverslip, and the filters. This interference causes an unwanted non-homogenous illumination pattern. A commonly used solution to bypass this problem is to use a multi-mode fibre which will scramble the light due to multiple allowed modes in the fibre. The diffuser in out setup is rotating, which changes the speckle and interference pattern temporally. Time-averaged, the light intensity distribution emitting from the rotating diffuser is similar to that of an incoherent light source.

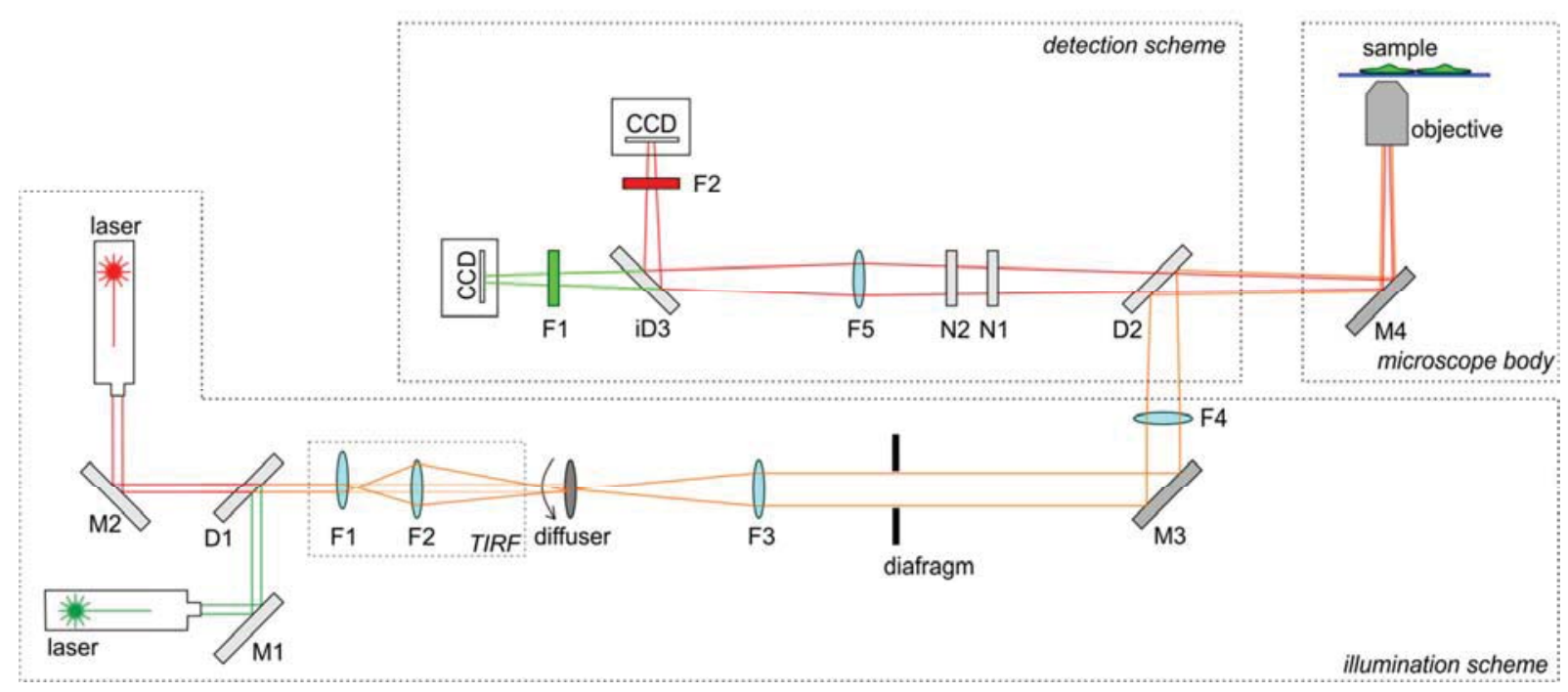

FIGURE 2.2 Schematic drawing of the dual-colour single-molecule sensitive TIRF microscope setup. D1 is a dichroic mirror that combines the laser light; before F1, an achromatic quarter wave plate is placed (not shown); F1 is a strong lens, and lens F2 focuses the laser beam onto the diffuser as a small off-center spot to obtain TIRF mode; both lenses can be quickly flipped in and out of the beam path; F3 is a $200 \mathrm{~mm}$ lens that collimates the light from the diffuser. A field-stop diaphragm is placed at the focal length of F3 and F4; M1-M4 are a silver coated mirrors; F4 is a $400 \mathrm{~mm}$ lens that focusses the diffuser image on the back-focal plane of the objective; the tube lens of the microscope body was removed; D2 is a dichroic mirror; N1 and N2 are laser line notch filters; F5 is an achromatic $300 \mathrm{~mm}$ lens that focusses the focal plane of the objective onto the emCCD cameras; iD3 is an image-quality dichroic mirror; F1 and F2 are band-pass filters. The camera recordings of the fluorescence from the green excitable dyes is called 'green channel' and the camera recordings of the fluorescence from the red excitable dyes is called 'red channel'.

\subsubsection{Widefield excitation scheme}

Following Köhler scheme illumination, the image of the light source was focused at the back focal plane by lens F3 and F4 (Fig. 2.2). When the image is coming from a point source, as the case for direct laser light, this results in only perpendicular rays at the sample side of the objective. Therefore the use of a diffuser is essential to implement a Köhler scheme, since in that situation, the light image is coming from an area, where the outer centre regions of the area lead to higher angle rays at the sample side. The field of view will be defined by the angles of the rays in the back focal plane. Since the maximum angle that the diffuser scatters to can be chosen, the field of view is determined by the choice of this angle.

To illustrate the scheme, and to design an optimal use of the angle of the diffuser and the focal lengths of the lenses, the light propagation through the setup is calculated using the thin lens approximation, which should be appropriate for lenses with a high focal distance. The objective is modelled as a $1.8 \mathrm{~mm}$ lens, whose focal length corresponds to the 100x magnification of an Olympus objective designed for the $180 \mathrm{~mm}$ tube lens in an Olympus microscope. Lens F3 is 
positioned at its focal length $\left(f_{3}\right)$ from the diffuser. Lens F3 and F4 are separated by the sum of their focal lengths $\left(f_{3}+f_{4}\right)$, and lens $\mathrm{F} 4$ is positioned at its focal length $\left(f_{4}\right)$ from the back focal plane of the objective (which is a distance $f_{\mathrm{Obj}}$ from the objective). The sample is located at the focal distance of the objective. Since the back port of the microscope, which is usually used for coupling of illumination light into the microscope, could not be used due to an existing optical tweezer modality of the microscope, the light was coupled from the bottom port of the microscope. Therefore the distance from lens F4 to the objective, and consequently its focal length, was spatially constrained to be at least $400 \mathrm{~mm}$.

To illuminate the complete camera area, a circular illuminated area with radius of $40 \mu \mathrm{m}$ is needed. The illumination radius is given by:

$$
r_{i l l}=f_{o b j} \cdot \tan \left(\alpha_{d} \cdot \frac{f_{3}}{f_{4}}\right)
$$

where $f_{O b j}$ is the focal length of the objective, $\alpha_{d}$ is the (half-width-half-maximum) angle of the diffuser, and $f_{3}$ and $f_{4}$ are the focal length of the corresponding lenses. Furthermore, to illuminate the sample up to the maximum angle $\alpha$ of the objective (related to its NA by $\mathrm{n} \cdot \sin \alpha$ ), the back focal aperture must be completely filled. The diameter of the laser beam is specified to be around 1 $\mathrm{mm}$ for the lasers used, which is therefore the diameter of the spot at the diffuser. We chose to magnify this spot by a factor of 2 onto the back aperture of the objective $\left(M=f_{4} / f_{3}, f_{3}=200 \mathrm{~mm}, f_{4}\right.$ $=400 \mathrm{~mm}$ ). To obtain the required illumination radius of $40 \mu \mathrm{m}$, the half-width half maximum (HWHM) of the diffuser angle has to be 2.5 degrees.

With the described choices for $f_{3}$ and $f_{4}$ the radius of the light path between lens F3 and F4 is $200 \tan 2.5^{\circ} \approx 9 \mathrm{~mm}$, which still fits within 1 inch sized components. Much larger focal lengths would require larger sized components. Also, for a much smaller choice for $f_{3}$, and therefore higher diffuser angles at equal illumination radius $r_{i l l}$, the maximum possible angle of the diffuser is quickly reached, since rays at large angles from the diffuser are blocked by the mounting construction of this rotating diffuser. Small perturbations in this design of the exact lens position were modelled by a perturbed propagation length of a few millimetres using transfer matrices describing the propagation of the light through the optical system; these small perturbations did not significantly alter the pattern or the ray angles of the illumination area. Therefore this design should be easy to align by hand. For the chosen design, the resulting rays at the various optical elements is shown in Fig. 2.3.
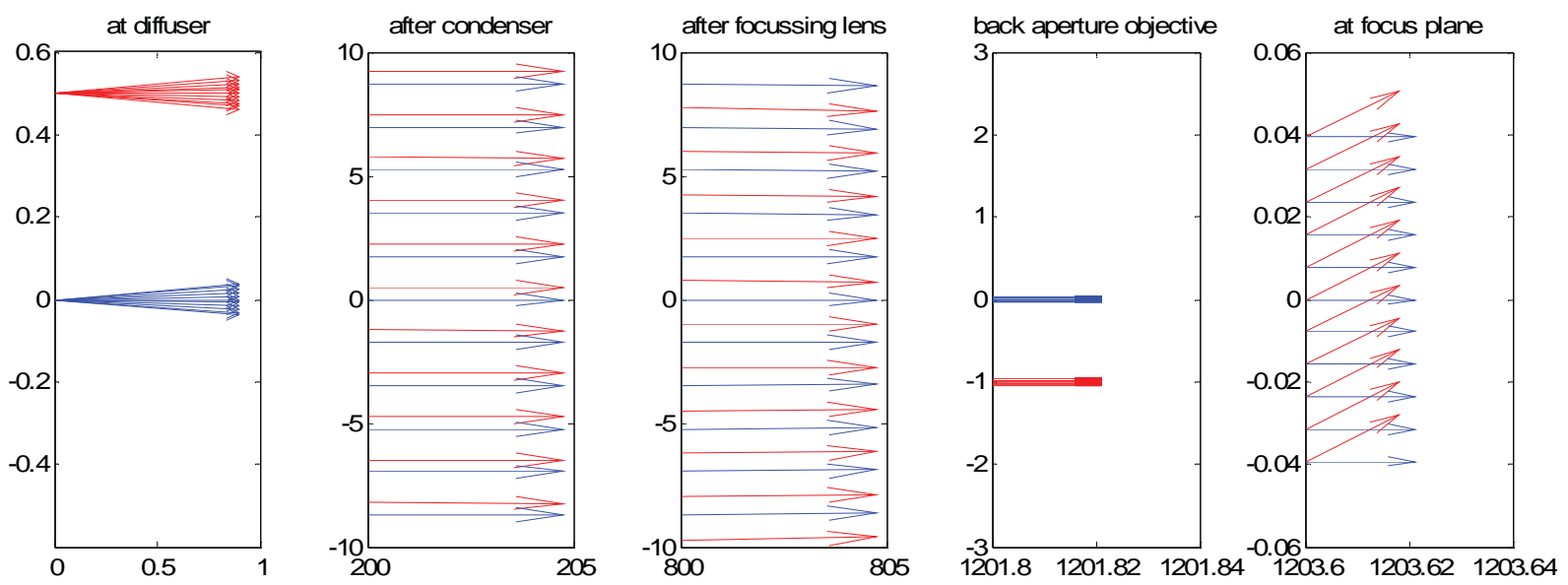

FIGURE 2.3 Light rays in the illumination part of the microscope setup for widefield illumination. The red arrows show the light rays originating from the outer regions of the Gaussian shaped light front, as the diameter of the light from the lasers was specified to be around $1 \mathrm{~mm}$. All distances are given in $\mathrm{mm}$. 
In a Kohler illumination scheme, a diaphragm can be used as a field stop diaphragm when it is placed at the focal length $f_{4}$ from lens $\mathrm{F} 4$, since there is a virtual image plane of the sample at that location. There is no need for a condenser aperture, as the amount of light can be changed by the illumination time. It is even impossible to incorporate this in the current scheme, as there is no accessible (virtual) image plane in the scheme.

\subsubsection{TIRF excitation scheme}

Total internal reflection fluorescence (TIRF) illumination can be obtained by a small extension to the layout of the optics as used for widefield illumination. As schematically drawn in figure 2.2, the TIRF extension can be obtained by the addition of a single small lens system to the layout. This lens system consists of two lenses (F1 and F2) where lens F2 is placed on a microstage to adjust the lens position in the lateral direction. The system is placed on a flip mount to be able to quickly switch between widefield and TIRF illumination. Lens F1 expands the laser beam, and F2 focuses it on the diffuser. The lens system effectively reduces the diameter of the laser beam on the diffuser plate, and translates the beam off the optical axis at the diffuser; this effects in a smaller and translated image at the back focal plane of the objective, which are the requirements to obtain TIRF illumination.

The radius of a laser beam at the interface of the diffuser is determined by the radius $r_{A}$ of the formed Airy disk pattern:

$$
r_{A}=\frac{0.61 \lambda}{N A}=\frac{0.61 \lambda \cdot 2 f_{2}}{D}
$$

with $\lambda$ the wavelength of the light, $f_{2}$ the focal distance of lens $\mathrm{F} 2$, and $D$ the diameter of the light beam at lens F2; therefore $r_{A} \approx 3 \mu \mathrm{m}$. However, since the diffuser has a certain thickness, the light will scatter within the thickness of the diffuser, effectively making a larger focal spot. After lens F2, the light is convergent with a maximum angle 9 given by:

$$
\sin \vartheta=\frac{D}{2 f_{2}} \approx \tan \vartheta=\frac{r_{e}}{L} \quad \mid f \gg D
$$

with $L$ the thickness of the diffuser, and $r_{e}$ the effective image spot radius. When $\mathrm{L} \approx 1 \mathrm{~mm}$, we obtain $r_{e} \approx 0.07 \mathrm{~mm}$, and therefore the image spot size is effectively given by $r_{e}$ (Eq. 6) and not by $r_{A}$ (Eq. 5). The image spot must be large enough to prevent damage from heat to the diffuser (intensity thresholds were not provided). At the same time, the beam width must be small enough to create a small enough spot at the back focal plane of the objective to obtain a full TIRF illumination. The spot radius of $0.07 \mathrm{~mm}$, which creates a spot with diameter of $0.14 \mathrm{~mm}$ at the back focal plane of the objective, resulted in a complete TIRF illumination without visible damage of the diffuser plate after good use of the microscope. The resulting light propagation is shown in Fig. 2.4.

\subsubsection{Emission scheme}

An FF494/540/650-Di01 dichroic mirror (Semrock) separated the excitation light from the emission light. To further remove unwanted excitation light, the emission light was filtered with an NF03-532/1064E and an NF02-633S notch filter (Semrock). A lens F5 ( $\left.f_{5}=300 \mathrm{~mm}\right)$ was used to focus the image on the CCD cameras. An imaging flatness quality dichroic mirror (FF640FDi01, Semrock) directed the different spectral parts of the image to the two cameras. The emission light from the green excitation dyes passed an FF01-580/60 bandpass filter (Semrock), and the light from the red excitation dyes passed an HQ680/60m bandpass filter (Chroma). 

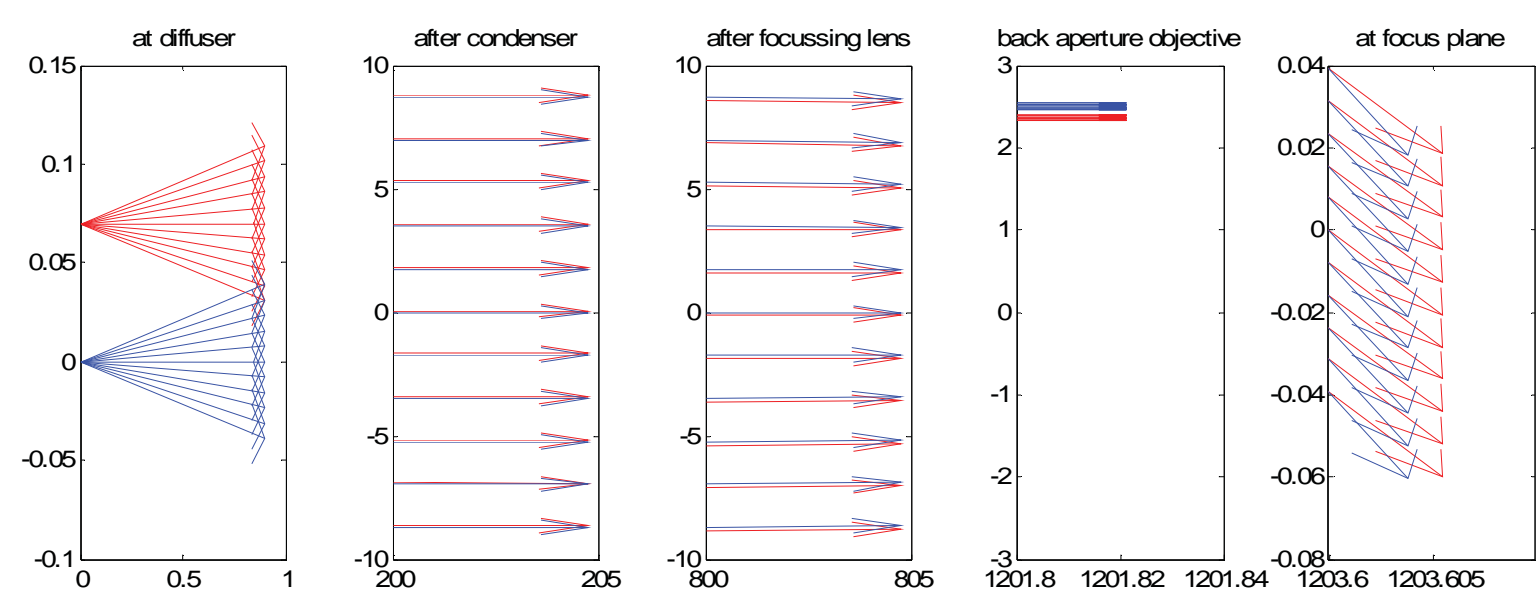

FIGURE 2.4 Light rays in the illumination part of the microscope setup for TIRF illumination. The red arrows show the light rays originating from the outer regions of the focused laser beam front. The effective diameter of the focused beam within the diffuser was assumed to be around $0.15 \mathrm{~mm}$. All distances are given in $\mathrm{mm}$.

Since the pixelsize of the camera should satisfy the Nyquist sampling criterion to record the point spread function (PSF), the magnification of the microscope should be designed correspondingly. The optimal performance for localization of single emitters might deviate from the Nyquist sampling due to pixelation and background noise ${ }^{12,15}$. When imaging freely rotating dipoles with a high NA (e.g. immersion) objective, the PSF deviates significantly from the classical Airy-shape of scalar diffraction theory. The best-fit Gaussian in these situations has a waist $\sigma$ approximately equal to $\sigma=0.25 \lambda / \mathrm{NA}^{16}$. For the emission light used here $(\lambda=580 \mathrm{~nm}$ and $\lambda=680 \mathrm{~nm}$ ), and the TIRF objective with an NA of 1.45 , this gives $\sigma_{580}=100 \mathrm{~nm}$ and $\sigma_{680}=117 \mathrm{~nm}$. The magnification is given by $M=f_{5} / f_{o b j}$ (lens F5 serves as the tube lens), such that with $f_{5}=300$ $\mathrm{mm}$, the magnification $M=150$. With a physical pixel size of the camera of $16 \mu \mathrm{m}$, the image pixel size becomes $107 \mathrm{~nm}$. This pixel size is similar to the Gaussian waist $\sigma$, therefore this magnification provides Nyquist sampling.

The dichroic mirror used to direct the emission light to the two cameras must have a very low curvature radius (i.e. image quality flatness). This is necessary to make an almost diffraction limited spot again from the reflected light at its image plane (Fig. 2.5). Mirrors with image quality are not available in a large range of wavelengths at the time of writing.

\subsubsection{Camera settings and excitation control}

The sample was only illuminated during signal acquisition. One camera acted as a master for the timing of the laser illumination and data acquirement by the cameras. The TTL trigger output of this camera is connected to the TTL input of the lasers and the TTL input of the other camera to ensure synchronized image recording. The cameras were controlled with the Andor Solis software. Furthermore the following settings were adjusted from the standard settings on both cameras:

- vertical pixel shift speed: $0.5 \mu \mathrm{sec}$

- readout rate: $10 \mathrm{Mhz}$ at $14 \mathrm{bit}$

- pre gain: $4.7 \mathrm{x}$

- EM gain: 300x

- kinetic series, without frame transfer.

- $512 \times 512$ pixels image size (full CCD chip)

The camera for the green channel was operated with an acquisition time of 9 ms (in TIRF mode for Dy 549), resulting in a frame rate of $25.85 \mathrm{~Hz}$ (limited by the camera readout rate). The camera for the red channel was operated with an acquisition time of $2 \mathrm{~ms}$ (in TIRF mode for CF633), and used an external trigger such that the frame rate was exactly identical to the other camera. 

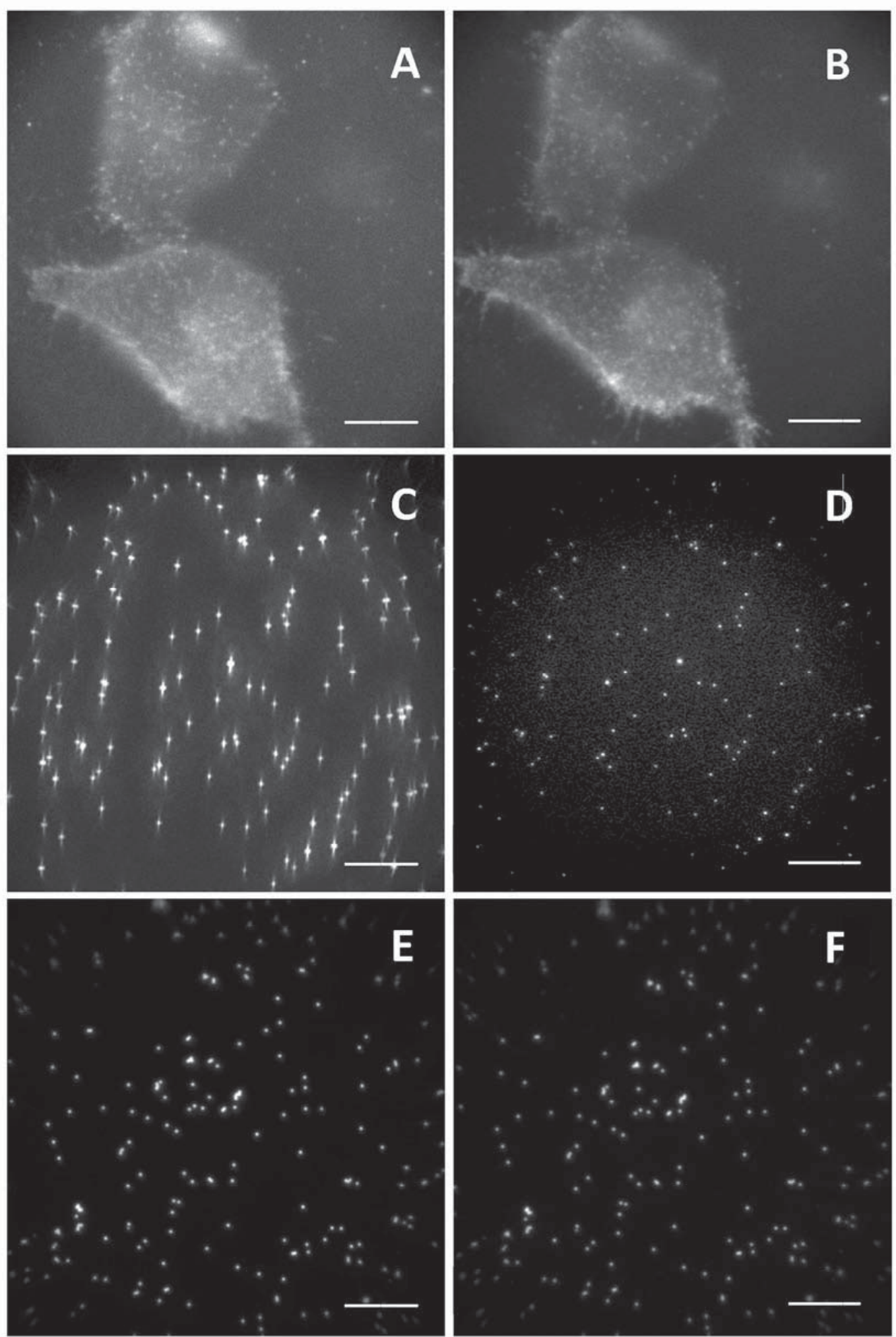

FIGURE 2.5 Fluorescence images of distortions caused by a normal quality dichroic mirror. Shown are single molecules in a cell in the distorted red channel (A), and in the undistorted green channel (B). In the undistorted channel filopodia and single molecules are better visible with less blur than in the distorted channel. Also shown are fluorescent beads in the distorted red channel (C), with corresponding undistorted green channel (D), and in an undistorted red channel with an image quality mirror $(E)$, and the corresponding green channel $(F)$. The distorted channel shows fuzzy vertical line blurring. The image quality mirror was used in the dual-colour experiments in this thesis. The scalebars are $10 \mu \mathrm{m}$. 


\subsection{Results: characterization and performance of the setup}

\subsubsection{General performance}

The characteristics and performance of the microscope realised are summarised in Table 2.1. The values are obtained from procedures described in more detail in the following subsections.

TABLE 2.1 Characteristics and performance of the microscope realized

\begin{tabular}{|l|l|}
\hline Property & Value \\
\hline Scaling & $119 \mathrm{~nm} /$ pixel \\
\hline Gain green channel & $49.9 \pm 0.1$ \\
\hline Gain red channel & $32.5 \pm 0.1$ \\
\hline Temperature of sample & $34.5-37.5^{\circ} \mathrm{C}$ \\
\hline Field of view diameter green channel (80-100\%) & $55 \mu \mathrm{m}$ \\
\hline Field of view diameter red channel (80-100\%) & $67 \mu \mathrm{m}$ \\
\hline Localization accuracy & $20-23 \mathrm{~nm} @ 70-100$ photons \\
\hline Thermal drift of sample stage & $<60 \mathrm{~nm}$ during 40 seconds \\
& (max 6 nm/second) \\
\hline Two-channel focal overlay precision & visibly complete overlap \\
\hline Two-channel lateral overlay precision & $<20 \mathrm{~nm}$ \\
\hline
\end{tabular}

\subsubsection{Temperature control}

The temperature control by the stage and objective heater was verified with a thermocouple. First the whole system was allowed to warm up for 1 hour. The clamps of the stage heater warmed up to the setpoint of $40{ }^{\circ} \mathrm{C}$, and the objective ring heater warmed up to its setpoint $38.5^{\circ} \mathrm{C}$. A Greiner Bio CellView dish (product \#627870) was put on the stage heater, which made contact only at the edges of the dish. The dish was filled with PBS at room temperature, and the objective was placed in contact with the dish. The temperature was measured in the centre of the dish directly above the objective, and at the edge of the dish. Without a lid on the dish, the temperature did not increase beyond $30{ }^{\circ} \mathrm{C}$ (centre) and $33{ }^{\circ} \mathrm{C}$ (edge). With a lid on the dish, and after 5 minutes warming up, the temperature reached $33^{\circ} \mathrm{C}$ (centre) and $36.5^{\circ} \mathrm{C}$ (edge), whereas after 30 minutes it got to $34.5^{\circ} \mathrm{C}$ (centre) and $37.5^{\circ} \mathrm{C}$ (edge). Therefore all measurements were made with the lid on the dish.

\subsubsection{Magnification}

The magnification was determined by measuring the effective pixel size. For this, a glass slide with line spacing of $10 \mu \mathrm{m}$ was used. The sample was recorded on both cameras, and the spacing was measured in pixels for both directions of the image:

- Green X: $117.5 \pm 0.5 \mathrm{~nm} /$ pixel;

- Green Y: $118.1 \pm 0.5 \mathrm{~nm} /$ pixel;

- Red X: $120.3 \pm 0.5 \mathrm{~nm} /$ pixel;

- Red Y: $120.5 \pm 0.5 \mathrm{~nm} /$ pixel.

For convenience, we further used $119 \mathrm{~nm} /$ pixel as the distance for one pixel.

\subsubsection{Excitation power and field of view}

The lenses and filters used reduce the power of the laser light reaching the objective. For the green laser $88 \mathrm{~mW}$ passed the objective, and for the red laser $47 \mathrm{~mW}$ passed the objective. The excitation field of view had an approximate diameter of $60 \mu \mathrm{m}$ (80-100\% intensity, Fig. 2.6) in the homogeneous parts of the excitation pattern, which resulted in an estimated 3.1 and $1.7 \mathrm{~kW} / \mathrm{cm}^{2}$ 
A

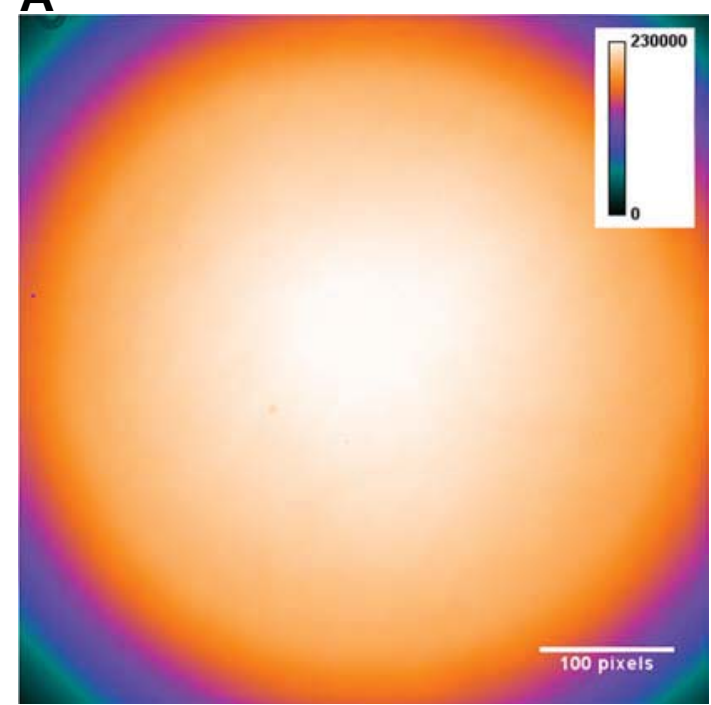

B

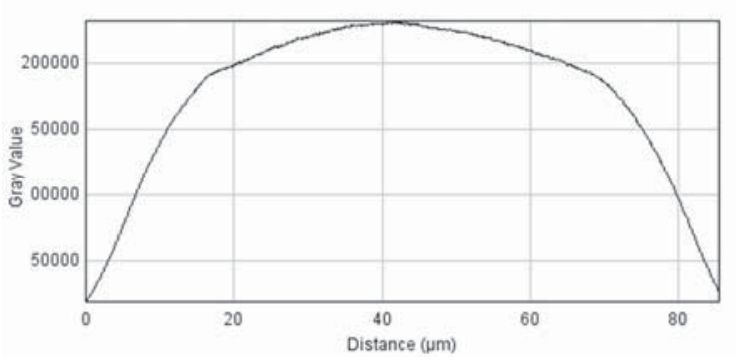

C

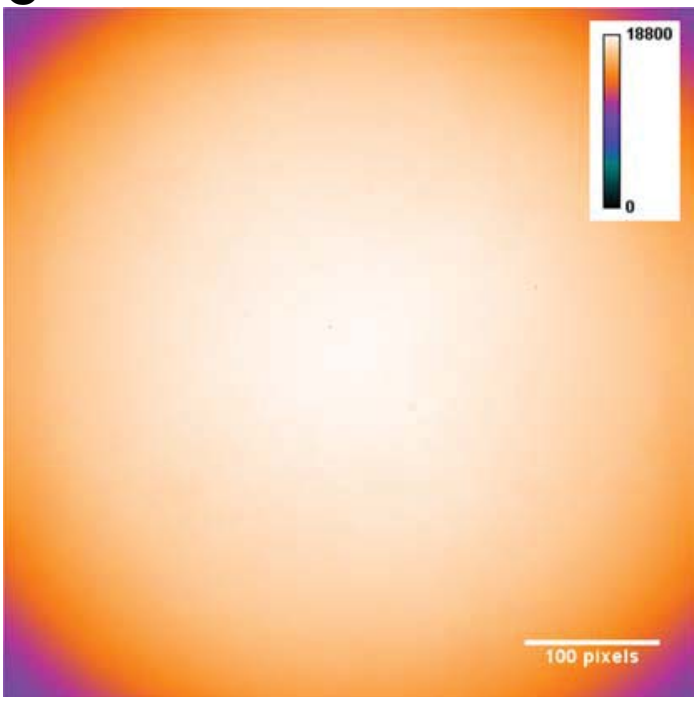

D

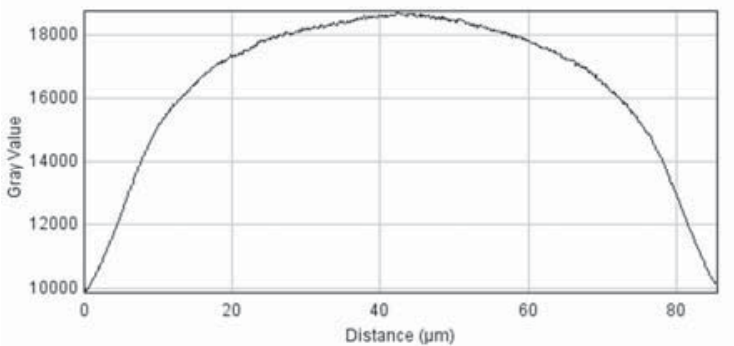

FIGURE 2.6 Fluorescence images in the green and red channel of the microscope of dyes in solution. The focus of the microscope was on the glass substrate. Images were recorded with the TIRF objective in widefield mode. Scaling is $119 \mathrm{~nm} /$ pixel. (A) The green channel was measured with Alexa 546 in solution. (B) Intensity plot over the diagonal of the green channel. (C) The red channel was measured with Alexa 647 in solution. (D) Intensity plot over the diagonal of the red channel. The channels differ in intensity due to different concentrations of dye, and different acquisition periods.

FIGURE 2.7 Calibration of the gain of the two EMCCD cameras performed by the mean-variance method. The slope of the line in this curve is equal to the inverse gain of the camera. A gain of $49.9 \pm 0.1$ was found for the camera recording the green-excitable dyes, and a gain of $32.5 \pm 0.1$ was found for the camera recording the red-excitable dyes.

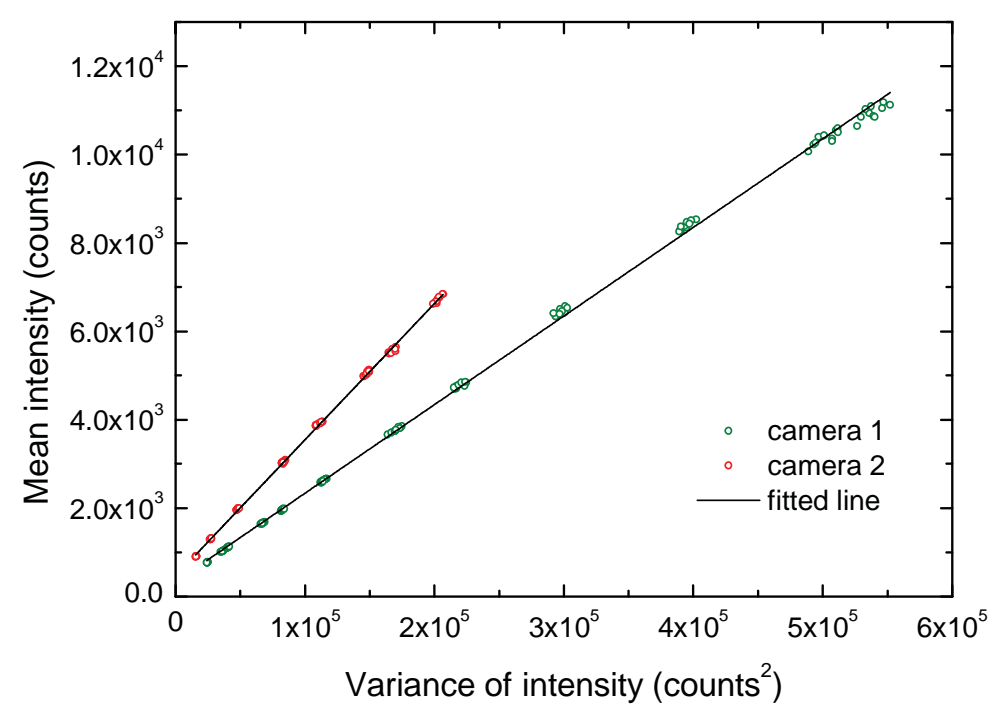


for the green and red excitation intensity respectively. The illumination diameter is smaller than the calculated diameter in Fig. 2.3, probably due to a smaller diffuser angle than 2.5 degrees FWHM as the angle of the diffuser plate is specified as the maximum angle.

Since the intensity and illumination pattern of TIRF illumination is strongly dependent on the exact angle of incidence at the coverglass-water interface, no illumination images are provided. We observed a clear difference in the amount of background in recordings of single molecules in cells between widefield and TIRF illumination.

\subsubsection{Characterization of the EM-CCD gain}

The mean-variance method $^{17}$ was used to determine the gain of the cameras to allow determination of the correct number of photons observed per molecule per frame. This number is needed to calculate the localization accuracy. Homogeneous images at different intensities were acquired by recording fluorescence of the relevant dyes in solution. The mean intensity of the pixels in each image is plotted against the variance of the pixels of the same image (Fig. 2.7). When a line is fitted through the points, the slope of this line is equal to the inverse gain of the camera. A gain of $49.9 \pm 0.1$ was found for the camera recording the green-excitable dyes, and a gain of 32.5 \pm 0.1 was found for the camera recording the red-excitable dyes.

\subsubsection{Localization accuracy and intensity per molecule}

The photon count per frame per identified molecule and its localization accuracy are key parameters to compare the performance of the microscope and the fluorescent probes used. The efficiency for fluorescence detection depends on the filter set used and the fluorescence spectra of the fluorophore itself. The number of photons collected per molecule per frame also depends on the acquisition time per frame. In Table 2.2 the typical fluorophores used to label the receptors studied in this thesis are listed, together with their excitation and emission efficiency in the microscope. The excitation efficiency is equal to the fraction of the maximum value of the excitation spectrum of the dye at the wavelength of the lasers, i.e. $532 \mathrm{~nm}$ for the green-excitable dyes, and $637 \mathrm{~nm}$ for CF633. The detection efficiency is the efficiency with which the emitted fluorescence passed the filter set used.

Apart from the number of photons, the localization accuracy depends on the background, which is strongly dependent on the particular cell, and the amount of pre-bleaching. Therefore the signal to background noise ratio, as often provided in the literature, will vary significantly depending on the exact conditions, for example the particular area in a chosen cell of a particular cell line at a particular growth stage in a particular buffer.

The localization accuracy is determined by the tracking algorithm used (see section 2.4.2) for all the localizations of the single molecules, and is based on the number and distribution of photons over the pixels. Although this provides a good comparison method for the photon-limited contribution to the localization accuracy, we emphasize that the localization accuracy as determined by the algorithm does not include other contributions such as biochemical effects. Flexible linkers are used between the two chimeras of fusion proteins (e.g. GFP is attached by a

TABLE 2.2 Efficiency of the excitation and detection of fluorescence in our setup, and acquisition time used for TIRF microscopy recordings.

\begin{tabular}{|lll|l|}
\hline BG-dye & $\begin{array}{l}\text { Excitation } \\
\text { efficiency }\end{array}$ & $\begin{array}{l}\text { Detection } \\
\text { efficiency }\end{array}$ & $\begin{array}{l}\text { Acquisition } \\
\text { Time (ms) }\end{array}$ \\
\hline Alexa 546 & 0.32 & 0.77 & 15 \\
\hline Dy 549 & 0.55 & 0.73 & 9 \\
\hline CF633 & 1.00 & 0.75 & 2 \\
\hline
\end{tabular}


variable length aminoacid chain linker to the wildtype protein), or between the substrate and the dye, which leads to rapid movements during the acquisition period. To get a mean localization accuracy that accounts for such effects, we would need to determine the offset in a curve of fitted diffusion values versus different timelags as determined using a CDF fit (see section 2.5.2). However, with this method only the mean value is obtained, and several assumptions have to be made about the diffusion system of the molecules for such an analysis, which complicates this analysis. Alternatively, the mean localization accuracy can be determined directly from a mean square displacement (MSD) curve of several trajectories, since the influence of multiple diffusion populations should average out when sufficient points are taken to compose the MSD curve. However, to circumvent averaging effects, it might be more reliable to determine the localization accuracy due to the photonic effect and estimate the biochemical effects, and convolve the two. Another component of the localization accuracy not regarded here is due to the movement of the molecule during the acquisition time.

The photon count and the localization accuracy differ between the dyes used and are sensitive to the exact illumination dose. Two typical intensity curves of immobile Alexa 546 molecules on glass in our setup are shown in Fig. 2.8. For comparison, the resulting signal to background noise ratio is around (45-30) / $1.5=10$, although this value fluctuates strongly. When all molecules in a typical TIRF recording are analysed by the tracking algorithm, the histograms of the photon count and the localization accuracy can be composed. Fig. 2.9 shows the results for the fluorophores Dy549 and CF633 in live MCF7 cells as prepared and measured according to the methods described in chapters 4 and 5 of this thesis. Since we make use of genetically encoded SNAP-tag technology, the fluorophore is rigidly bound inside the SNAP-tag domain $(20 \mathrm{kDa})$ which is attached via a short flexible amino-chain linker to the protein of interest itself. We therefore estimate that the localization accuracy due to the linker is around $5 \mathrm{~nm}$, which has a negligible contribution compared to the photon-limited effect on the total mean localization accuracy. To achieve a localization accuracy of $20 \mathrm{~nm}$, more than the theoretically necessary 36 photons per molecule were needed (section 2.2.2.2). This might be caused by the motion blur during the acquisition period, and the fluorescence background. Our values for the localization accuracy of mobile molecules are similar to earlier reported values for immobilised clusters on fixed cells (MSD = $0.0088 \mu \mathrm{m}^{2}$, corresponding to $\sigma_{\mathrm{xy}}=23.5 \mathrm{~nm}$ ), whereas in that study the average number of photons detected per single molecule per frame was higher (225 photons $)^{18}$.

\subsubsection{Thermal drift}

Another important performance measure of the setup is the drift of the sample during the measurement. In single-molecule tracking studies, the drift is often not of much concern since the diffusion of the single molecules and even the apparent diffusion due to the localization accuracy are often larger than the apparent diffusion due to the drift of the microscope system. However,

FIGURE 2.8 Typical intensity (photon count) curves of single fluorescent molecules. The standard deviation of the two recorded background signals are 1.5 and 2.2 photons.

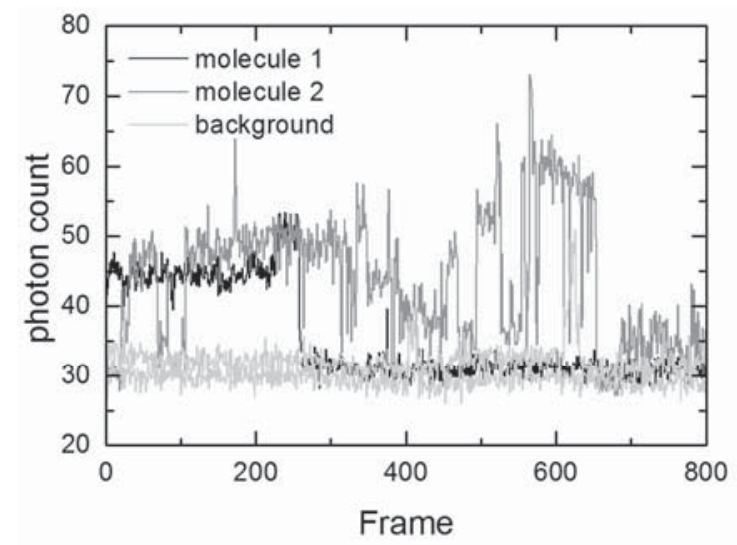



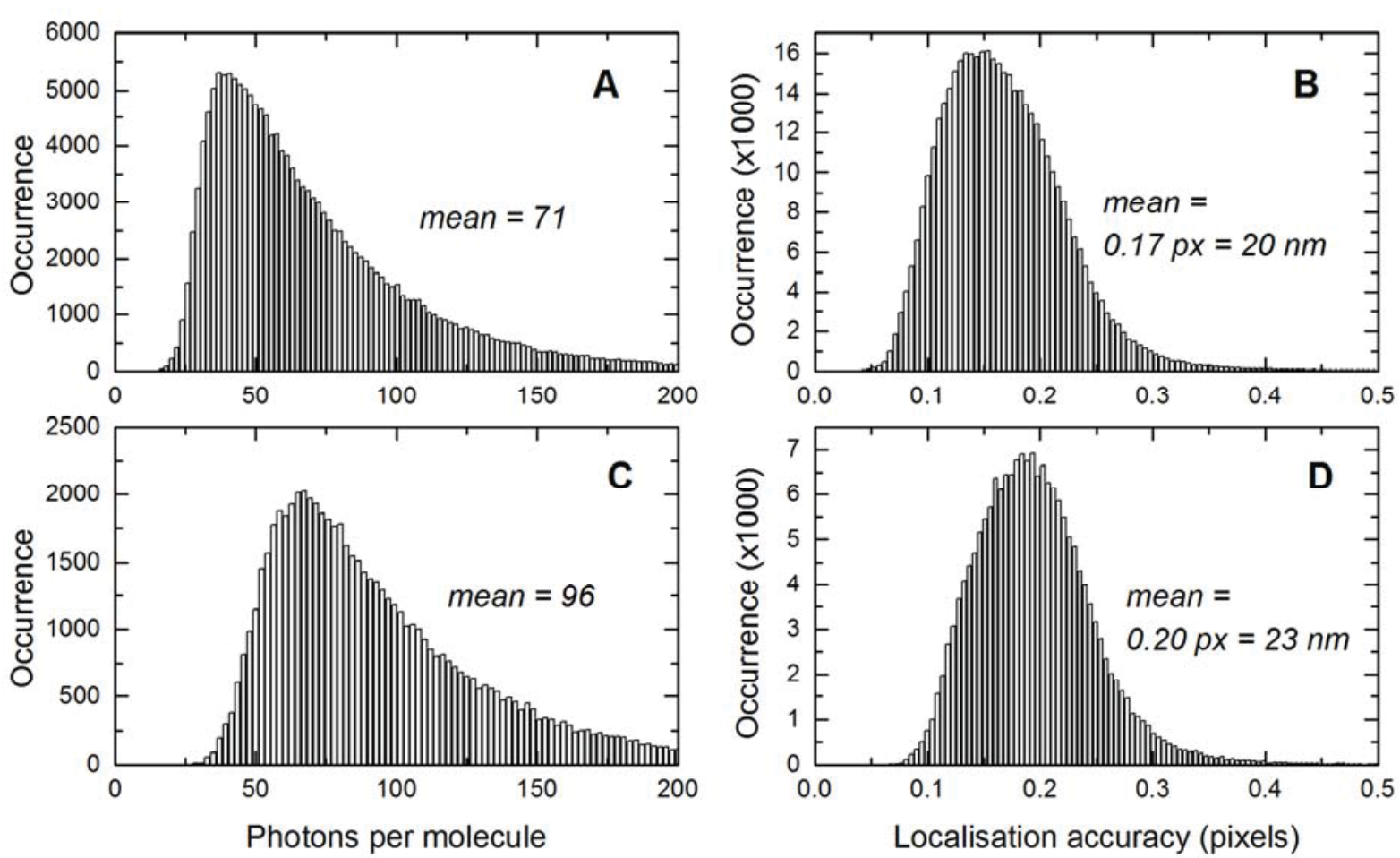

FIGURE 2.9 Histograms of the photon count per frame per molecule and the localization accuracy of the molecules for a typical TIRF recording in live cells in this thesis. (A) Photon count for Dy 549. (B) Photonlimited localization accuracy for Dy 549. (C) Photon count for CF 633. (D) Photon-limited localization accuracy for $\mathrm{CF}$ 633. The localization accuracy is given as $\sigma_{x y}$ which is the error in one direction. The error in other directions is typically the same. Biochemical effects have not been taken into account in these localization accuracies.

the extension of the analysis of single-molecule data to include reconstructed high-resolution images (chapter 3 ) requires that the sample is not drifting much more than the resolution of the reconstructed high-resolution images. Since typical recordings are 30-60 seconds, which is relatively short compared to, for example, the STORM/PALM imaging duration, we are not hindered by long-term drifts. However, in many single-molecule studies there is a need to measure at physiological temperatures $\left(37^{\circ} \mathrm{C}\right)$, which requires heating equipment inevitably resulting in temperature gradients in the setup. Temperature fluctuations are a major cause of drift in setups due to thermal expansion $\left(240 \mathrm{~nm} / \mathrm{cm} /{ }^{\circ} \mathrm{C}\right.$ for aluminium, i.e. 2 pixels).

First we determined the long-term drift in our microscope when the heating equipment was turned off (Fig. 2.10A). To this end, we measured the average displacement of several fluorescent beads immobilised on a glass substrate (imaged at $0.1 \mathrm{~Hz}$ ) and subsequent tracking (see section 2.4.2); subsequently, a moving average filter (5 frames) was applied to the integrated displacements. We determined the drift during the duration of a typical experiment (40 seconds) by imaging at $26 \mathrm{~Hz}$, and without the heater turned on this drift was less than 120nm (Fig. 2.10B). Next the heating equipment was turned on, and we allowed the system to stabilise for at least one hour. The drift dramatically increased (Fig. 2.10C).

When the heater construction is mounted at two positions, such as the default attachment of the stage heating plate, the expansion has to go in the perpendicular direction, which results in bending and thereby an even larger expansion in the perpendicular direction. As we found that the default attachment of the heating plate using hinges was not optimal concerning the thermal drift, we drilled a hole in the heating plate, and fixed the plate with one screw on only one side of the plate to the mechanism that allows the stage to move. This did not change the thermal contact 
to the sample. This construction resulted in the lowest drift level (Fig. 2.10D). In this situation, $95 \%$ of the positions recorded (4 times standard deviation) were within $60 \mathrm{~nm}$ (in one direction) during a typical measurement period.
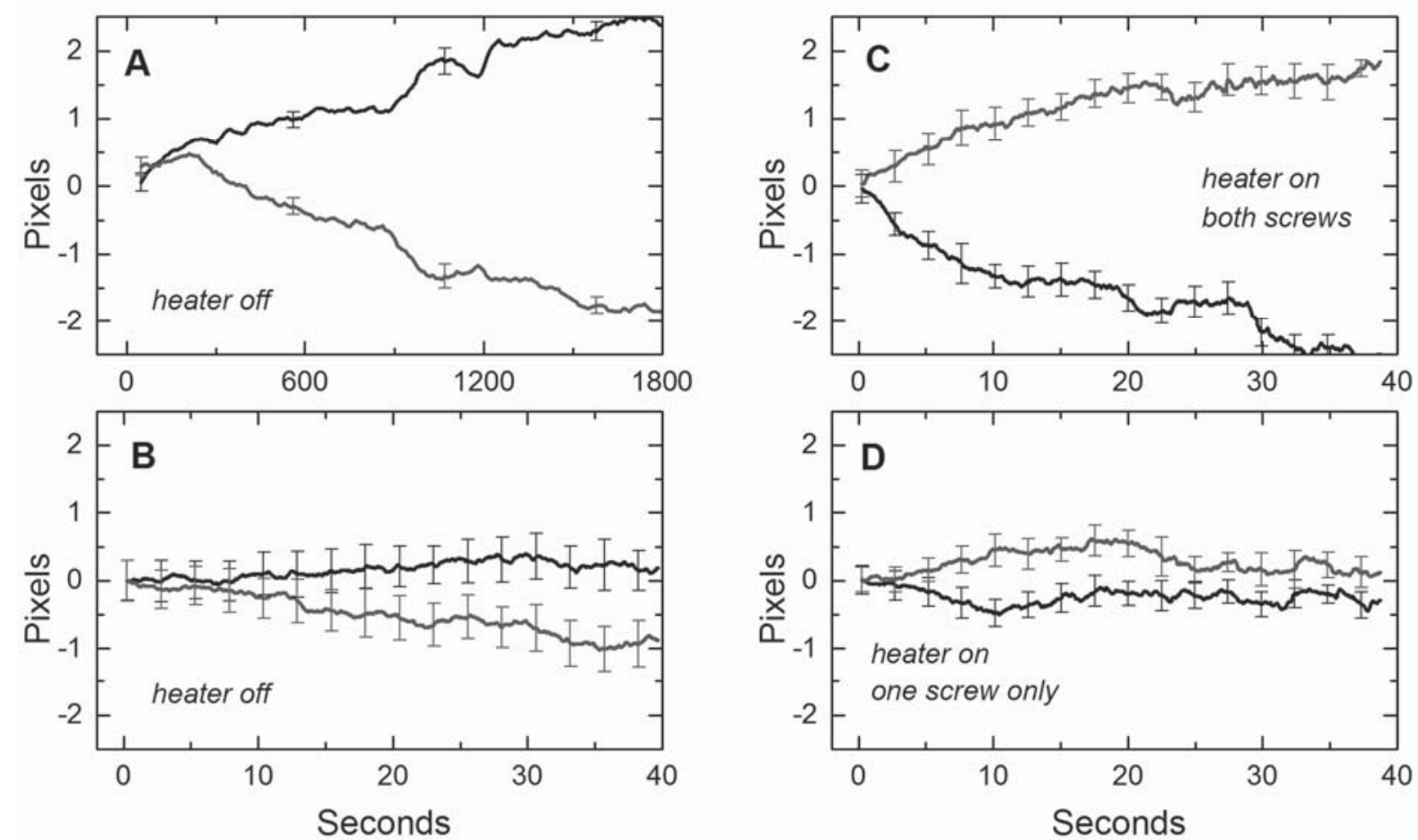

FIGURE 2.10 The drift of the microscope in distinct situations. Shown are the integrated mean displacements of all fluorophores which were immobilized on a glass substrate. (A) The long-term drift is limited to about $250 \mathrm{~nm}$ (2 pixels) in half an hour for each direction when the stage plate heater is off. (B) The drift along the duration of a typical experiment is around $100 \mathrm{~nm}$ in total when the stage plate heater is off. (C) When the plate stage heater is turned on, and the microscope is allowed to warm up for 1 hour, and the heater is screwed on two sides to the stage itself, the drift is significantly increased and would not allow proper super-resolution recordings. (D) When the plate stage heater is only attached with one screw on one side to the stage itself, there is no extra observable drift compared to the situation where the heater is turned off.

\subsubsection{Dual colour overlay}

Since we used two cameras to measure fluorescent signals at distinct emission wavelengths, these cameras had to be aligned to record the same area of a sample in the same focal plane. We shortly discuss the methods employed to overlay the images of both cameras.

\subsubsection{Focal overlay}

Since the imaging plane of the microscope is at a fixed distance after the imaging lens (Fig. 2.2), both cameras need to be placed exactly at this distance from the lens to have an equal focal plane in the sample. The precise alignment was performed by moving the dichroic mirror iD3 (Fig. 2.2), which only changes the distance of camera 2 with respect to the imaging lens. The alignment was correct when the shape of a fluorescent bead (immobilized on a glass substrates as a sample) was found to be equal on both cameras while focussing through the sample (Fig. 2.11). The shape was quantified by drawing a region of interest (ROI) around the fluorescent bead, and monitoring the standard deviation of the pixel intensities within this ROI; this was more sensitive to the focal distance than the maximum or mean intensity values. After focussing on single molecules in the green channel, we always obtained a perfect focus in the red channel as well, confirming the precise focal overlay. 
A

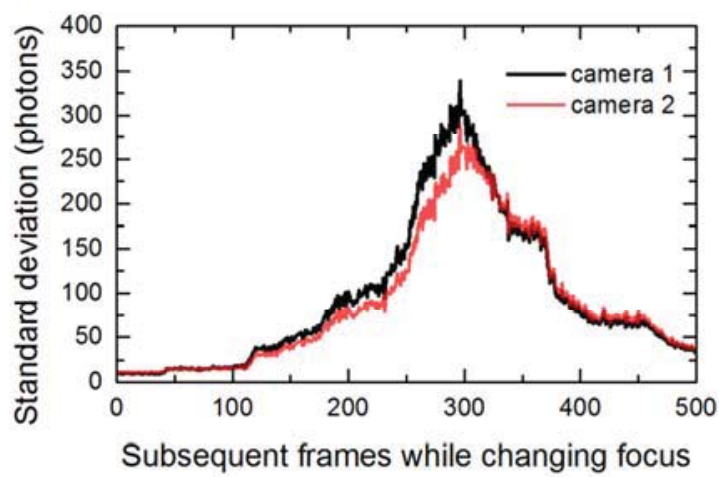

B

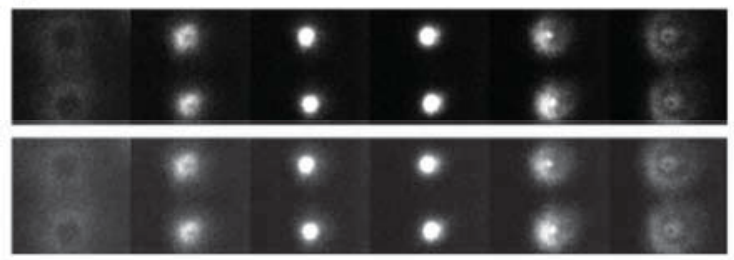

FIGURE 2.11 The two cameras were positioned to have an equal focal plane in the sample. (A) The shape of a fluorescent bead is represented by the standard deviation of the pixels of the areas shown. (B) The areas around the fluorescent beads at different focus positions (left to right) for camera 1 (upper image series) and camera 2 (lower image series).

A

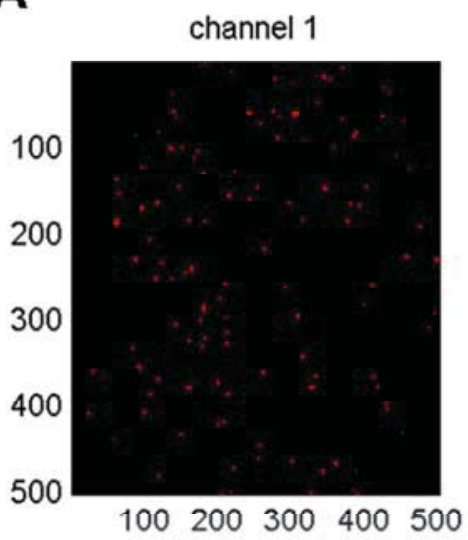

B

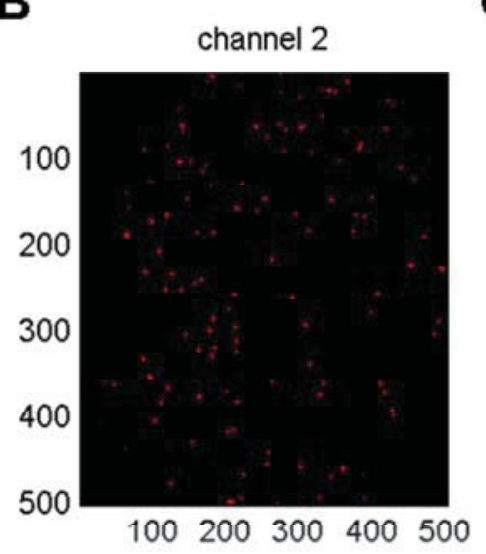

E

D raw overlay $(502 \times 502)$
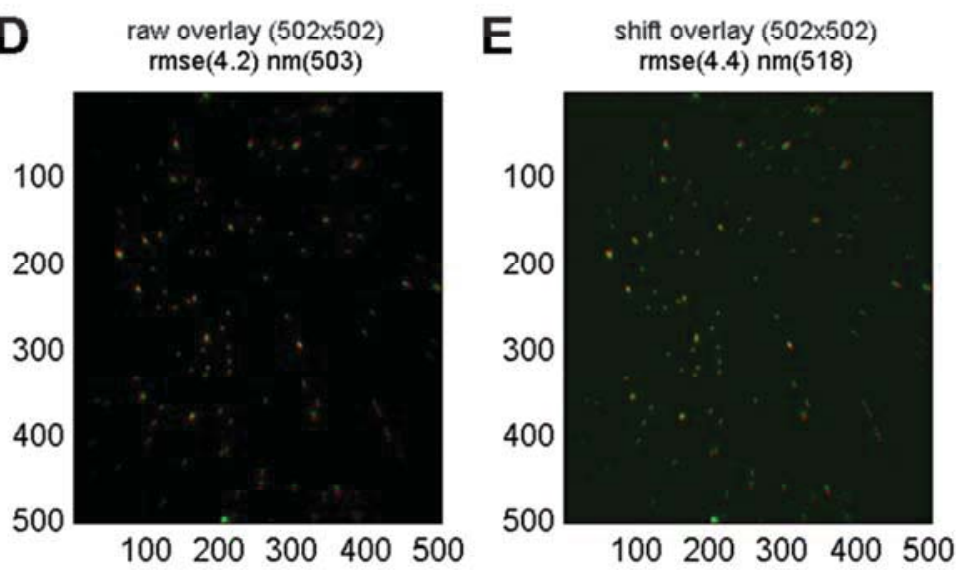

C ch1 's' ch2 'o' ch2est ' $x$ '

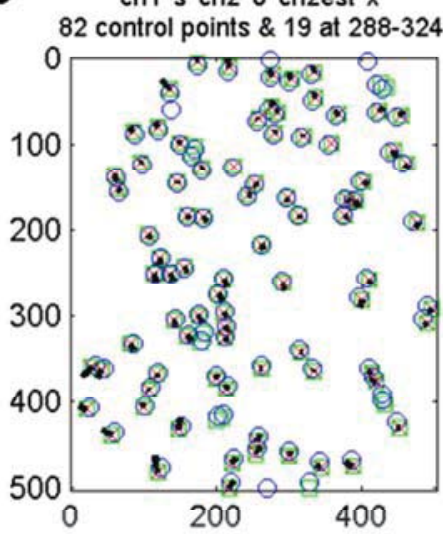

F interp overlay $(484 \times 484)$

tre(0.16) fre $(0.15) \mathrm{nm}(17.9)$

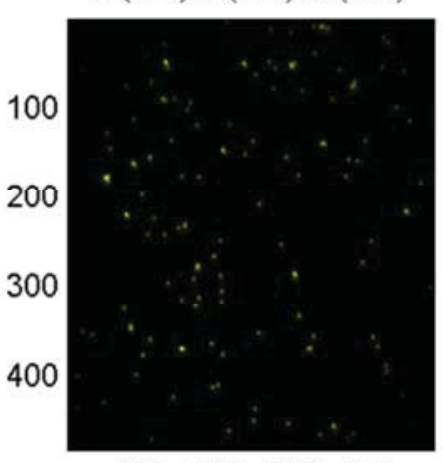

$100200300 \quad 400$

FIGURE 2.12 Implementation of the channel overlay used. The MatLAB function transforms the coordinates of the trajectories in one channel into the other channel. For this, images of beads are required for the two channels $(A$ and $B)$, from which the transformation matrices are determined such that a sub-pixel overlay can be performed. $(C)$ The detected beads are used as control points to calculate the transformation. $(D)$ The twochannel overlay without any transformation of the second channel. (F) The two-channel overlay when the second channel is only transformed using lateral shifts. $(F)$ The two-channel overlay when the second channel is transformed using the full transformation calculated. 


\subsubsection{Spatial overlay}

Apart from a focal plane overlap, the cameras were positioned to have minimum lateral shift. A correction is still needed to perform precise sub-pixel overlays. The tracking software used, developed by others (section 2.4.2), includes a sub-pixel overlay function included to transform the coordinates of the trajectories in one channel into the other channel. This function requires images of fiducial markers (fluorescent beads) in both channels to find the necessary translation and scaling, and overlays the two channels (Fig. 2.12). The achieved overlay accuracy for the example shown is $17.9 \mathrm{~nm}$ (standard error between position of control points in channel 1 with estimated overlay position of control points in channel 2). This accuracy is sufficient to detect dimers by correlated motion analysis, as that method detects two receptors as dimers when they are within $50-100 \mathrm{~nm}$ from each other. For non-tracking purposes, such as visualization of twocolour images or videos in this thesis, we performed the channels overlay by an implementation in MatLAB utilizing the find_affine_trans and affine_trans functions in the DIPimage toolbox (http://www.diplib.org).

\subsection{Tracking single molecules: from recordings to trajectories}

In this section we discuss how we obtained the trajectories of single molecules from the microscopy recordings. We first applied an image filter on the image series of the recordings to reduce the background and remove the intensity offset. Then the filtered image series were processed by the tracking algorithm to obtain the trajectories.

\subsubsection{Pre-filtering of raw video data}

Background signals with a low spatial frequency can be adequately filtered, and will improve the tracking quality because the tracking algorithm cannot deal with background offsets, and needs the actual number of photons emitted by a single molecule. When background signals are present, the segmentation part of the algorithm will also detect background signals as single molecules when the pixel intensity of the background signals is above the detection threshold. The actual number of photons in each pixel is needed to perform a correct localization which uses a maximum likelihood approach. The total number of photons emitted by a single molecule is also needed to determine the localization accuracy. Therefore the background signals, e.g. the camera baseline and out-of-focus signals, should not contribute to the single molecule signals. We designed an image filter that minimizes the amount of signal removal from single molecules while maximizing the removal of background signals. The pre-filtering makes it unnecessary to determine the baseline of our images, since the filtered images should not have any baselines or offsets.

\subsubsection{Methodology of the filter applied}

Since we record fluorescent images of fluorophores with different emission wavelengths, the size of the single-molecule images is also different and is defined by the radius of the point spread function $\left(R_{P S F}\right)$. Therefore the filtering procedures were scaled with the PSF radius. The PSF radius in our setup can be best estimated using $R_{P S F}=0.25 \lambda / N A$, as explained in more detail in section 2.2.4. We did not use Kalman or other temporal-based filtering, since the molecules of interest move (in a random fashion). The filtering procedure is performed on each individual frame of an image timeseries.

First a parabolic opening filter with a radius equal to the PSF radius was applied on the original image (Fig. 2.13A), after which the obtained image was smoothed with a Gaussian kernel with a radius 3 times as large as the PSF radius. This image was then subtracted from the original image. This filtering operation removed higher frequency terms, but not as high as signals close to the PSF radius (Fig. 2.13C). For a Gaussian signal, the filter described above is actually equal to a 
Gaussian smoothing filter with radius 3 times the PSF radius. However for images with background signals, it is not the same, and the Gaussian smoothing filter then subtracts more at the spots and less from the background as compared to the opening based filter (Fig. 2.13D), which is undesirable.

To remove lower frequencies as well, the filtered image obtained underwent one more smoothing filter, defined by a Gaussian kernel with a radius 5 times as large as the PSF radius. The thereby obtained image is the pre-filtered image (Fig. 2.13B), and this image is used as input for the tracking software. The pre-filtered image clearly shows much less background signals and suppressed vignetting effects compared to the original image while maintaining the distinctive signals of single molecules.
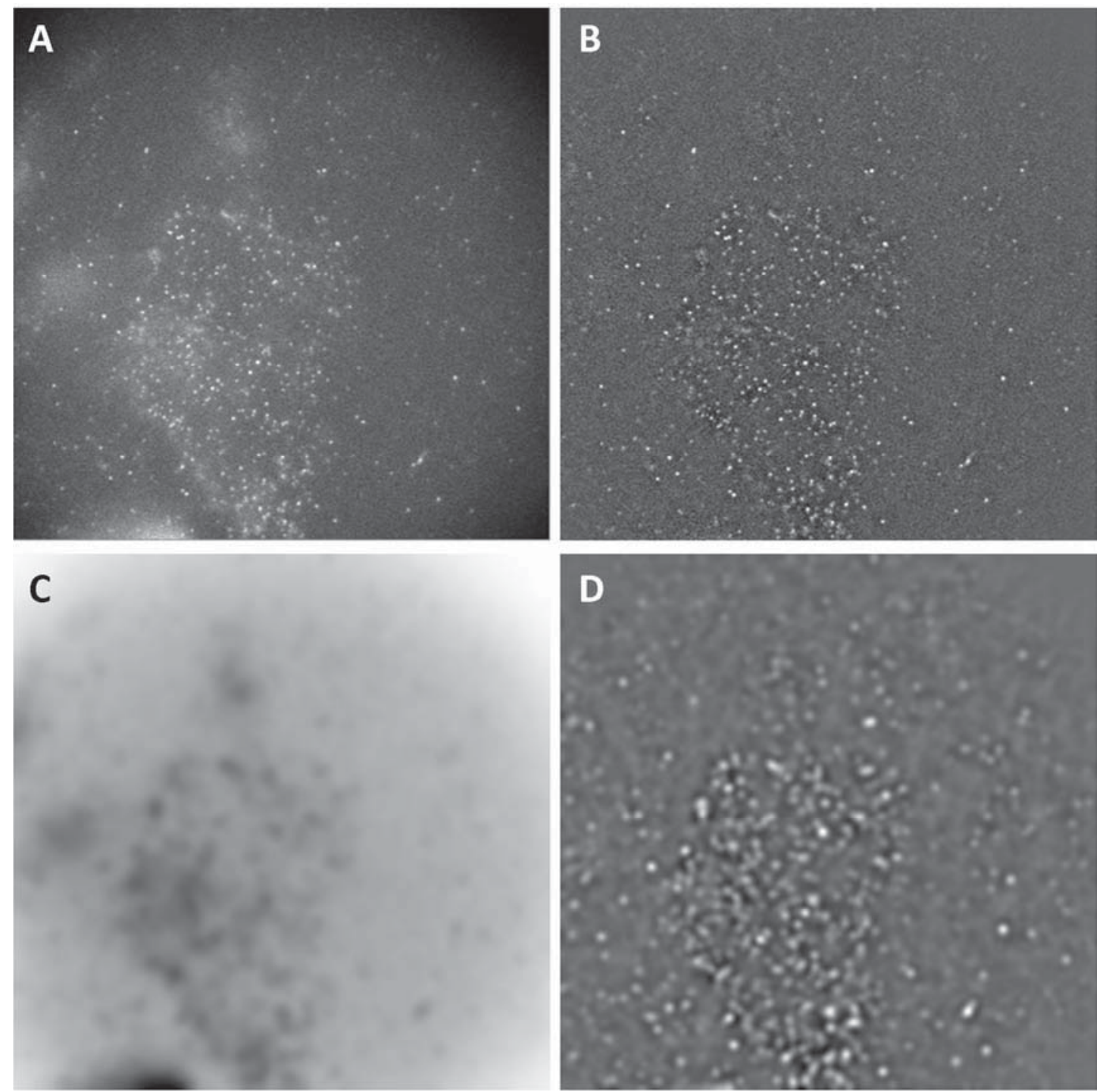

FIGURE 2.13 Properties of the first step of the filtering procedure. (A) Original image as recorded before any filtering. (B) Image result of the pre-filtering procedure. (C) Difference of the filtered and original image. The filtering procedure described reduces the background (black zones) but leave signals at the scale of the point spread function of the microscope untouched (no black dots with the size of a single molecule can be observed). This is an ideal filter for this application as baseline offsets must be removed, but the single molecule signals must be untouched for correct localization. (D) Difference image of the filtering procedure described and a purely Gaussian smoothing filter. The difference in both filters is that signal from single molecules stay relatively better preserved with the filtering procedure described, as the image is white (more preserved) at the location of single molecules. 


\subsubsection{Tracking software}

In this thesis, all measurements were tracked with the previously described tracking software developed in the group of $\mathrm{K}$. Lidke ${ }^{4,19}$. This software had our preference over other tracking software $^{3,20}$, because it uses GPU based calculations to perform the fitting of the molecules which is much faster. The analysis of a typical 1,000 frames 512x512 pixel image series takes about 5 minutes on our computer. Furthermore it is based on the same solid mathematical basis on the origin of diffusion as other advanced software ${ }^{20}$. One disadvantage of most and also this tracking software to date is that the linking process is optimized for only one diffusion constant, and as such the fact that protein molecules can exhibit multiple diffusion states is not taken into account. This can cause incorrect linking of subsequent positions when two molecules are close by.

\subsubsection{Settings}

The tracking software comes with a list of parameters to set up the software to find the most probable trajectories. The settings are divided in a subset used to perform the Gaussian fitting (localization) of signals from single molecules, and in a subset to perform the linking and reconnection of the positions determined in the localization also referred to as the linking cost matrices. The settings that we modified from the default settings are listed, together with the value used for the tracking and our comments for that setting (Table 2.3, in Appendix). An important property of diffusion is the wide spread of possible step sizes (distance travelled in one frame). To limit the range (search distance) for linking, we make use of the fact ${ }^{7}$ that $99 \%$ of the step sizes are smaller than $2.55 \cdot \sqrt{4 D \tau_{\text {lag }}}$.

\subsubsection{Extensions to original Lidke group software}

We made several modifications to the tracking software as received, which are incorporated in newer versions by the developers. These issues include mostly the pre-processing of areas in which to perform the localization (Fig. 2.14), and issues when no background images are provided as input.
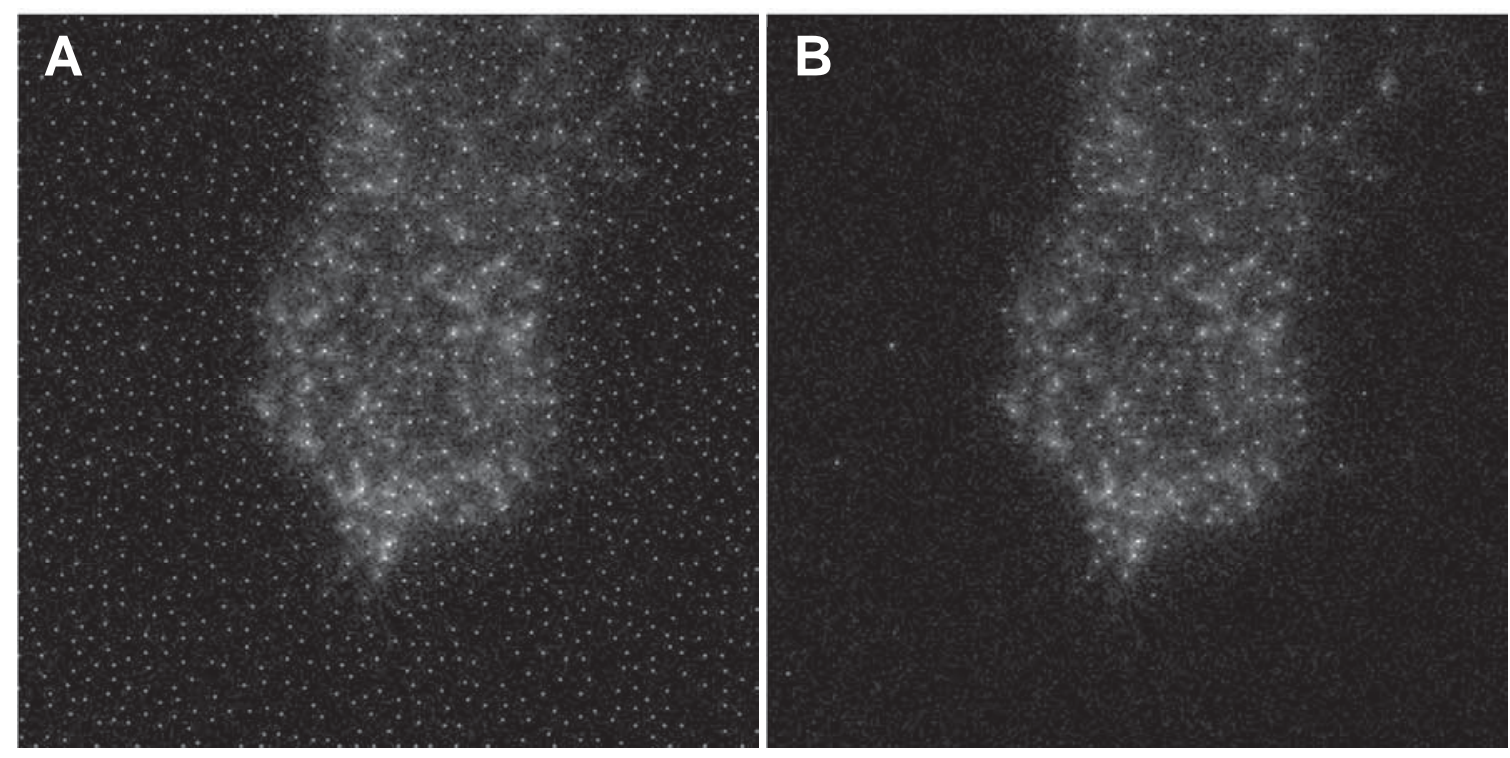

FIGURE 2.14 The FindBox function determines the areas (subimages) to feed to the fitting routine to perform the localization. (A) The function as received was not correctly selecting the areas of interest, and resulted in over-localizations. Although these localizations are later removed due to a threshold on the minimum photon count, this significantly slowed down the software. (B) The areas of interest found using our updated function as incorporated in version BETA4 and higher. 
Furthermore we made a few extensions to the software. To better visualize the tracking process we made a function which overlays the original video recording with coloured circles around the single molecules localized, where each colour represents another trajectory (Fig. 2.15A). This made it easier to verify that the correct settings were used in the tracking software. Furthermore, we made a simple GUI which makes it user-friendly to load microscopy recordings, stored as FITS images, and determine an appropriate intensity threshold for molecule detection (Fig. 2.15B). The real-time visualization of the effects of a certain intensity threshold made it much faster to choose a proper threshold. These extensions are freely available online (or from the author).
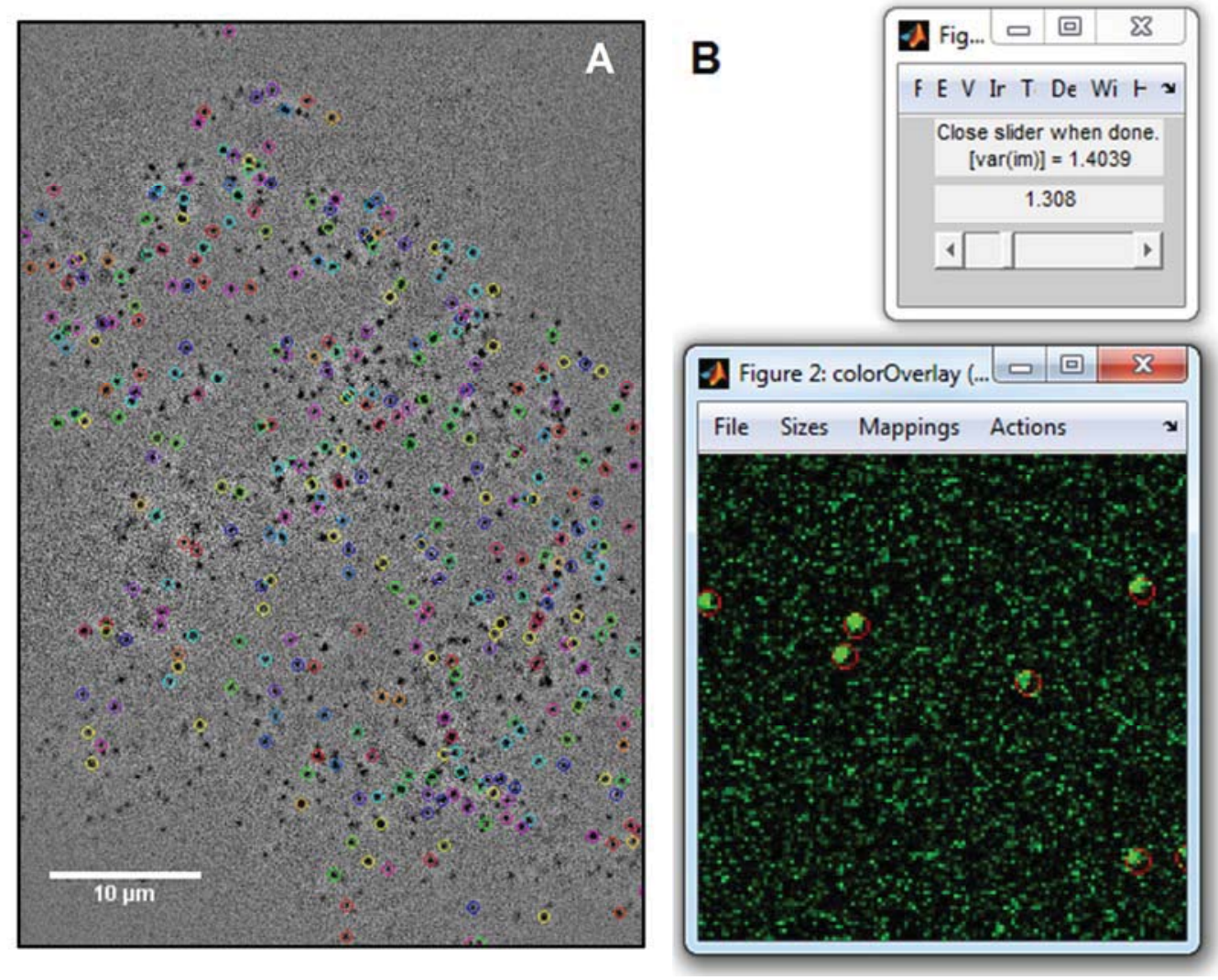

FIGURE 2.15 Two extensions were written to facilitate easier use of the tracking algorithm. (A) One extension to the tracking software encircles the detected molecules in the microscopy recording where colours are used to differentiate trajectories. (B) Another extension facilitates a user-friendly and automated loading of FITS files to the tracking software.

\subsection{Analysis of trajectories}

Trajectories of proteins are analysed to gain information about these proteins, the dynamics of membrane components, or kinetics of protein-protein interactions ${ }^{21,22}$. Initially, analysis of singlemolecule tracking data considered complete trajectories using mean squared displacement (MSD) curves. For a homogenous mobility type, the shape of the MSD curve contains information about the nature of the diffusion, e.g. pure, confined, or hop diffusion ${ }^{7,23-25}$. Later, it was realized that the organization of the plasma membrane was much more complex than previously believed. For instance, (transient) spatial zones where the motion of a protein deviates from free diffusion were revealed $^{24,26-28}$. This led to analysis methods to detect confined motion, i.e. motion hindered by (transient) confinement zones ${ }^{7,29-32}$. Although transient confinement or slowed diffusion are closely related, confinement is actually defined as pure diffusive motion restricted by boundaries which cannot be crossed. The confinement area should be of reasonable size such that the unconstrained (free) diffusion within this area can still occur. The diffusion constant is however 
the same as for unrestricted motion. Confinement is therefore different from slow(ed) diffusion, since slowed diffusion is unconstrained (free) diffusion but at a lower diffusion constant. There is no consensus yet on the exact type of motion proteins exhibit.

Mean squared displacement (MSD) curves of full trajectories are still often used to analyse the motion of proteins ${ }^{7,33-36}$. We emphasize that these curves are only suitable for homogeneous motion. It is very easy to misinterpret the curves and derive wrong conclusions when the studied system contains more than one motion type. Since the MSD curve is composed of averages of all distances, these transient phases make the MSD curve models prone to misinterpretation if the dwell time in a given population is shorter than the trajectory (Fig. 2.16). Related to this, the reliability of higher time lag points should not be overestimated ${ }^{25}$, as discussed in more detail later. Finally, conclusions from MSD curves must always be tested against unconstrained diffusion, as the randomness of normal diffusion may result in apparent anomalous diffusion ${ }^{37}$.

Within a trajectory the motion of a protein may not be homogeneous, but involve multiple types of motion caused by transient molecular interactions, for example transient directed motion, confinement, and multiple populations of free diffusion ${ }^{3,29,38-40}$. When it was realized that proteins show transient effects, local methods were developed that considered sub-trajectories (segments) of a trajectory ${ }^{3,25,41,42}$. These methods are however hampered by the limited number of positions within one segment to obtain accurate diffusion coefficients or confinement strengths.

Our scope is analysing trajectories from single-molecule video data, although it is possible to analyse single-molecule data on its own, for instance cluster size based on the histogram of intensities of spot. Using image correlation techniques it is also possible to obtain the characteristics of two diffusion components without the need for frame to frame tracking of the spots. These particle image correlation spectroscopy (PICS) approaches have been described in detail ${ }^{43,44}$. Such methods do not yield spatial information, and we will therefore not describe these methods in more detail.

A

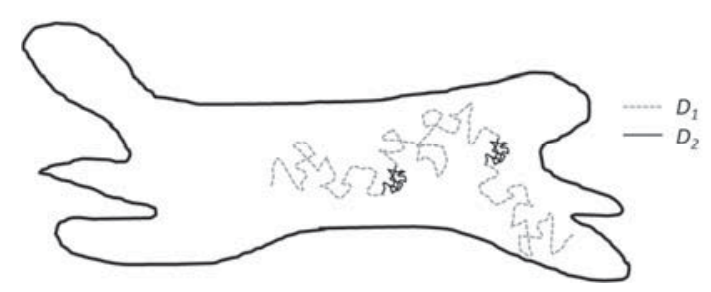

B

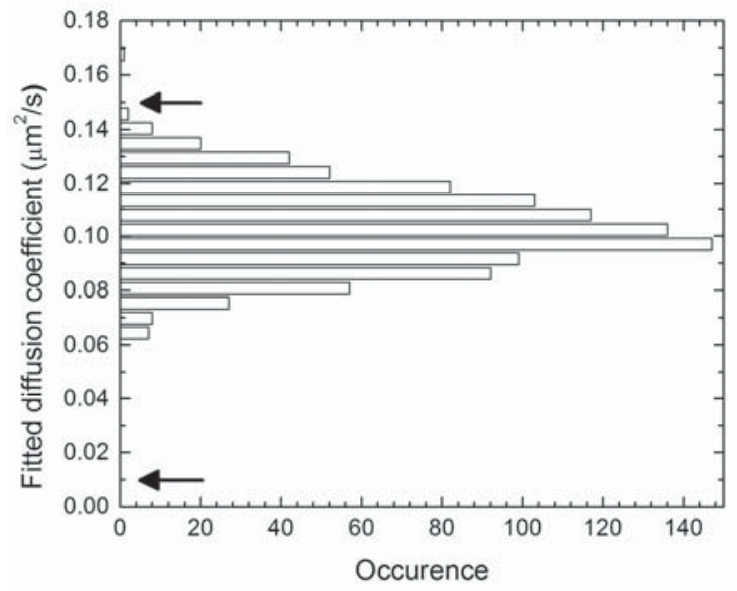

FIGURE 2.16 A two-population diffusion system cannot be analysed using a conventional full trajectory MSD method. Such analysis leads to erroneous characterizations for this motion system. (A) Cartoon to illustrate a typical trajectory of a single protein on a plasma cell membrane, displaying two different diffusion coefficient periods within one trajectory. $(B)$ The apparent distribution of diffusion values found by an incorrectly applied full trajectory MSD analysis. Here two-population diffusion with diffusion coefficient of 0.15 and $0.01 \mu \mathrm{m} / \mathrm{s}^{2}$ (values indicated in the graph with arrows) was simulated for trajectories consisting of 500 frames. The trajectories are analysed using the conventional full-trajectory MSD method, where the first 4 points of the MSD curve were used following a "rule of thumb" to fit the diffusion constant. The found diffusion constants and the apparent spread are both incorrectly describing this two-population situation. The MSD analysis should only be used for homogeneous (one-population) motion. The discussion further in the supplementary material discusses the accuracy of determining the diffusion constant for a one population system using the MSD method. 


\subsubsection{Mathematical description of Brownian diffusion}

The mathematical properties of Brownian motion are mentioned in almost every probability theory textbook in the context of a Markov process. The first published study about Brownian motion was from Bachelier in 1900 in the context of stock market fluctuations, although the physical phenomenon of Brownian motion is usually attributed to Brown, and was further elaborated by Einstein. A few years later, Wiener had established a lot of the mathematical framework to describe numerous facets of Brownian motion. We will only repeat the essentials of Brownian motion here, much more details can be found elsewhere ${ }^{45,46}$. As the energy for Brownian motion of molecules is thermal energy, the acting forces make particles move according to the heat equation as described by Fick's second law, which can be rewritten for the case of one particle as:

$$
\frac{d}{d t} p(r, t)=D \cdot \nabla^{2} p(r, t)
$$

where $p(r, t)$ is the probability density (or concentration for multiple particles), and the Laplacian operator $\nabla^{2} p(r, t)$ denotes the second derivative in space of the density, which logically equals the change over time in the density due to the conservation of mass. By integration over $r^{2}$ the cumulative distribution function (CDF) of squared displacements $\Delta R^{2}$ is obtained ${ }^{30}$, and yields for two-dimensional diffusion:

$$
C D F\left(\Delta R^{2}, t\right)=1-\exp \left(-\frac{\Delta R^{2}}{4 D t}\right)
$$

The corresponding (probability) density function (PDF) is obtained by simple differentiation, but almost never used, as scalar squared displacements cannot be used to define a trajectory, since we need vector displacements to do so.

The PDF of displacements is described by the normal distribution and can be derived using the Bernoullian distribution ${ }^{47}$, and is given by:

$$
\begin{aligned}
& P D F(\Delta x, t)=\frac{1}{\sqrt{4 \pi D t}} \exp \left(-\frac{\Delta x^{2}}{4 D t}\right) \text { for } 1 \text { dimension } \\
& P D F(\Delta R, t)=\frac{1}{4 \pi D t} \exp \left(-\frac{\Delta R^{2}}{4 D t}\right) \text { for } 2 \text { dimensions }
\end{aligned}
$$

When more populations with distinct diffusion coefficients are present, the multiple diffusion states are linearly added to the distribution.

\subsubsection{Cumulative Density Function}

In this thesis we assume that the motion of membrane proteins can be described by two states of Brownian diffusion, and we will call these the fast and the slow population. To find the two diffusion constants $D_{1}$ and $D_{2}$ and the fraction $\alpha$ of the first population, we calculate the cumulative distribution function of squared displacements for the complete set of trajectories ${ }^{30}$. Whether the assumption that the motion can be attributed to two populations is correct can be checked by looking at the residuals of a fit of the cumulative distribution function (CDF). Using the complete distribution yields insights into the behaviour of the entire population of single molecules, without ensemble averaging effects. As long as there is a large enough dataset of displacements to build a reliable CDF, it is a straight-forward and reliable method to find the global diffusion coefficients and their fractions. Fig. 2.17B shows an example of a CDF fit for motion with two clearly separated diffusion populations. 

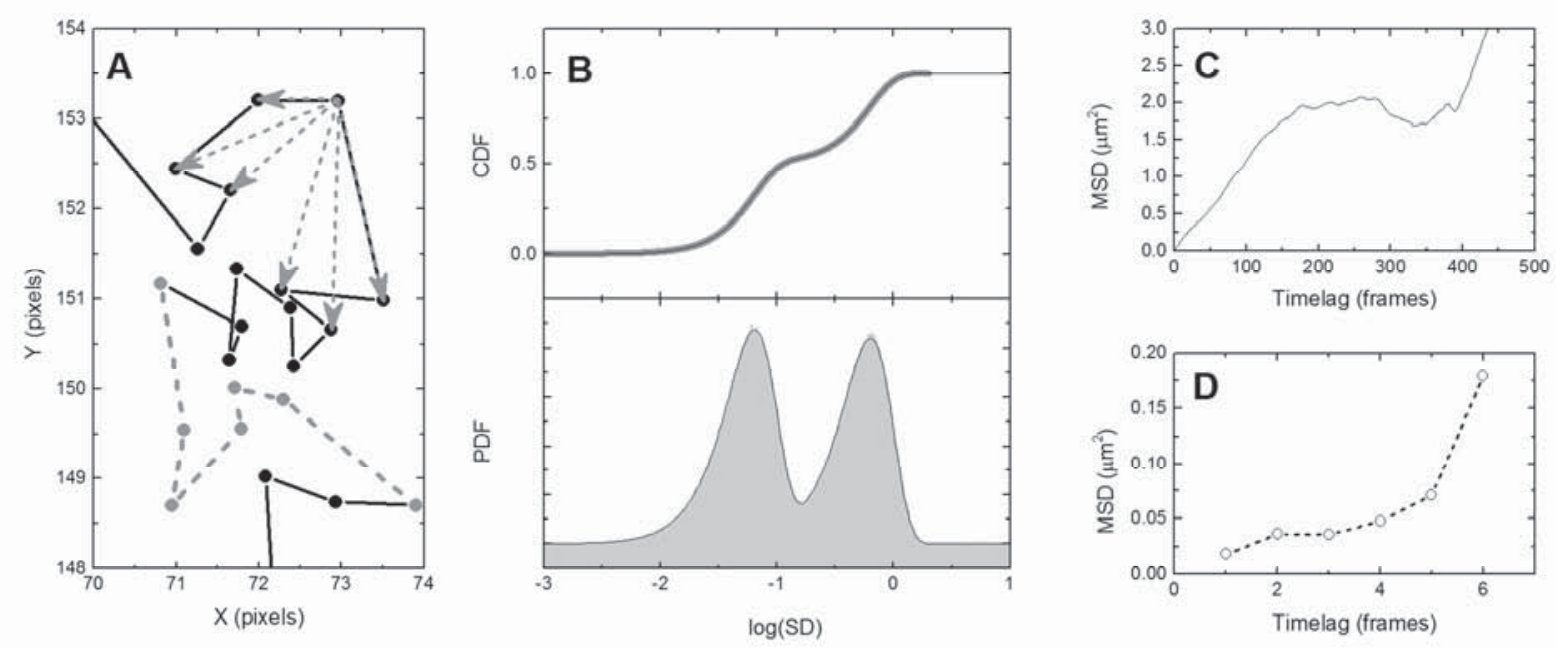

FIGURE 2.17 Illustrations of various trajectory and segment analysis methods. (A) Simulated two-population diffusion trajectory and examples of selected information for the relative confinement and the gyration method on a trajectory segment. Relative confinement detection takes the variance of distances from the centre of the segment (indicated by the grey arrows). The gyration radius depends on the variance and covariance of the coordinates (segment indicated in grey with a dashed line). $(B)$ Cumulative distribution function (CDF) values and fit of squared displacements from a set of trajectories with two diffusion coefficients (upper), and the corresponding probability density function which is the derivative of the CDF (lower). The simulated values are in grey, the fit is drawn in black. (C) A conventional full trajectory MSD curve from pure one-population diffusion (first 500 frames shown). (D) A windowed MSD provides an instantaneous diffusion coefficient for all timepoints of a trajectory by performing an MSD analysis only on a segment (the window, as indicated with dashed grey lines in panel $A$ ) of the trajectory, and sliding this window through the whole trajectory.

For the two dimensional case, the cumulative distribution function (CDF) for the squared displacements $(\Delta R)^{2}$ for a time lag $\tau=n \cdot \Delta t$ for two diffusion components is given by:

$$
\begin{gathered}
C D F\left(\Delta R^{2}(\tau)\right)=1-\alpha \cdot \exp \left(-\frac{(\Delta R)^{2}}{4 D_{1} \tau+2\left(\sigma_{x}^{2}+\sigma_{y}^{2}\right)}\right)-(1-\alpha) \\
\quad \cdot \exp \left(-\frac{(\Delta R)^{2}}{4 D_{2} \tau+2\left({\sigma_{x}}^{2}+{\sigma_{y}}^{2}\right)}\right)
\end{gathered}
$$

where $\alpha$ is the fraction corresponding to the motion with diffusion coefficient $D_{1}$. To deal with the localization inaccuracy in the exponent, we determine $D_{1}, D_{2}$ and $\alpha$ for the time lags corresponding to 1 and 2 frames, and fit the exponential terms:

$$
\begin{gathered}
C D F\left(\Delta R^{2}(1)\right)=1-\alpha_{\tau=1} \cdot \exp \left(-\frac{(\Delta R)^{2}}{4 \widehat{D}_{1, \tau=1}}\right)-\left(1-\alpha_{\tau=1}\right) \cdot \exp \left(-\frac{(\Delta R)^{2}}{4 \widehat{D}_{2, \tau=1}}\right) \\
C D F\left(\Delta R^{2}(2)\right)=1-\alpha_{\tau=2} \cdot \exp \left(-\frac{(\Delta R)^{2}}{4 \cdot 2 \widehat{D}_{1, \tau=2}}\right)-\left(1-\alpha_{\tau=2}\right) \cdot \exp \left(-\frac{(\Delta R)^{2}}{4 \cdot 2 \widehat{D}_{2, \tau=2}}\right)
\end{gathered}
$$

which yield the uncorrected diffusion coefficients for each timelag, for example $\widehat{D}_{1, \tau=1}=D_{1, \tau=1}+$ ${\sigma_{x y}}^{2}$ since ${\sigma_{x}}^{2}+{\sigma_{y}}^{2}=2{\sigma_{x y}}^{2}$, and similarly $2 \widehat{D}_{1, \tau=2}=2 \cdot D_{1, \tau=2}+{\sigma_{x y}}^{2}$. Now the estimated 
diffusion coefficient for the first (and similarly for the second) population corrected for the localization error is:

$$
D_{1}=2 \widehat{D}_{1, \tau=2}-\widehat{D}_{1, \tau=1}=\left(2 D_{1, \tau=2}+{\sigma_{x y}}^{2}\right)-\left(D_{1, \tau=1}+{\sigma_{x y}}^{2}\right)=2 D_{1, \tau=2}-D_{1, \tau=1}
$$

For the fraction $\alpha$ we take the average of the values $\alpha_{\tau=1}$ and $\alpha_{\tau=2}$. In the simulations these two values did not differ by more than a few percent. We have used a linear least squares method to fit the CDF to the data.

\subsubsection{Mean squared displacement (MSD)}

The most straightforward way to determine the diffusion coefficient is by using the mean squared displacement (MSD) versus time lag curve ${ }^{1}$. This provides an estimate of the diffusion coefficient, and also confinement ${ }^{7}$, but the method requires that within the complete trajectory there is only one type of homogeneous motion. In short, the MSD is usually defined as the average of all squared distances between points within a certain lag time $\tau=n \cdot \Delta t$, with $\Delta t$ the time-delay between consecutive frames, and $n$ the interval of frames over which the distance is measured and averaged (Fig. 2.17C). For intervals larger than 1 frame, usually all available distances of a given duration $n \cdot \Delta t$ are included, such that the distances are not statistically independent. Yet this way of averaging gives less variance to the average squared displacement value compared to taking only the independent distances ${ }^{48}$. For pure Brownian motion, the relation between squared displacements $(\Delta R)^{2}$ and the diffusion coefficient is a linear relation:

$$
\operatorname{MSD}(\tau)=\left\langle\left(\Delta R_{\tau}\right)^{2}\right\rangle=4 D \tau+2\left({\sigma_{x}}^{2}+{\sigma_{y}}^{2}\right)=4 D \tau+4{\sigma_{x y}}^{2}
$$

where $\sigma_{x y}$ is the standard deviation of the localization inaccuracy in one dimension, which is independent of the time lag. The estimated diffusion coefficient $D$ is found from fitting a line through the points at the different lag times in the MSD curve. We emphasize that it is not straight forward how to perform the fitting of the MSD curve to obtain diffusion values (detailed below).

\subsubsection{Windowed MSD}

Typically, the MSD curve is made up from all positions in a trajectory, which cannot resolve local changes in the diffusion coefficient. Windowed MSD tries to give the local or instantaneous diffusion coefficient at each timepoint of a trajectory by performing the MSD analysis on small segments of the trajectory. First an MSD curve is composed for $w$ subsequent positions in a trajectory, and the estimated $D$ value is obtained from the first three points in the curve for this segment. This value is taken as the measure $W$. Then the MSD curve is made for the next subsequent positions, until the full trajectory has been slid through, and $\mathrm{D}$ values have been obtained for each segment (Fig. 2.17D). The use of a moving window makes it possible to detect temporal changes in the mode of motion on the order of the window size. The resolution is limited by the averaging nature of the method, since reducing the window size means that the MSD curve is made up from fewer points, therefore increasing the statistical uncertainty of the fitted diffusion coefficient.

\subsubsection{Accuracy of MSD methods and CDF fitting to obtain a one population diffusion coefficient}

A recurring question is which points in the MSD curve can still be considered reliable. Certainly the variance of larger time lags gets increasingly larger, such that the points of larger time lags do not provide any reliable information. In the literature the first $10 \%$ points of the curve are often assumed to have not too much variance in their values ${ }^{2}$. However, the analytical expression for 
one population Brownian motion for the variances has been derived ${ }^{48,49}$. Following this expression, Michalet discussed what the optimal number of points is to be taken into the fit for determining the diffusion coefficient ${ }^{25}$. The optimal number of points depends on the ratio $\beta=\sigma^{2} /(D \Delta t)$, with $\sigma$ the standard deviation of the localization inaccuracy. In the limit of no (or relatively small) localization inaccuracy, i.e. for small $\beta$, it was shown that the most accurate value for $D$ is obtained by fitting with only the first two points of the MSD curve. This result was already noted earlier ${ }^{7}$. However, since we consider two population diffusion systems which have both high and low diffusion constants and correspondingly both low and high $\beta$ values, we do not readily know the optimal number of points of the MSD curve that should be used in the fit. We have checked how the correctness of the fit depends on the number of points in the MSD curve using simulations for a one population system (Fig. 2.18), and discuss a two population system in chapter 3.

A

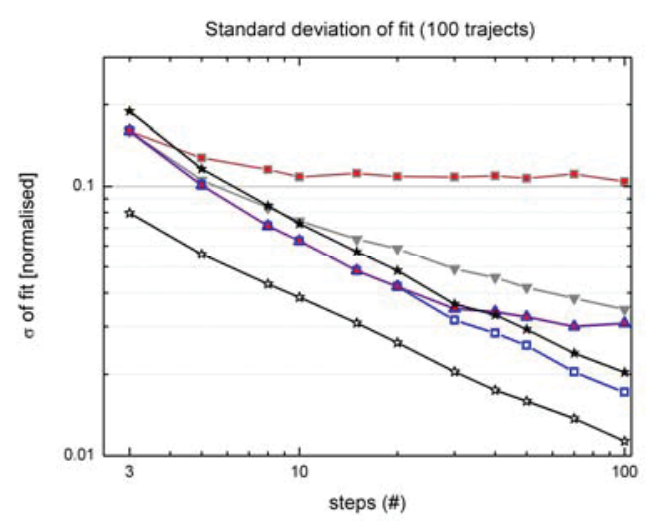

C

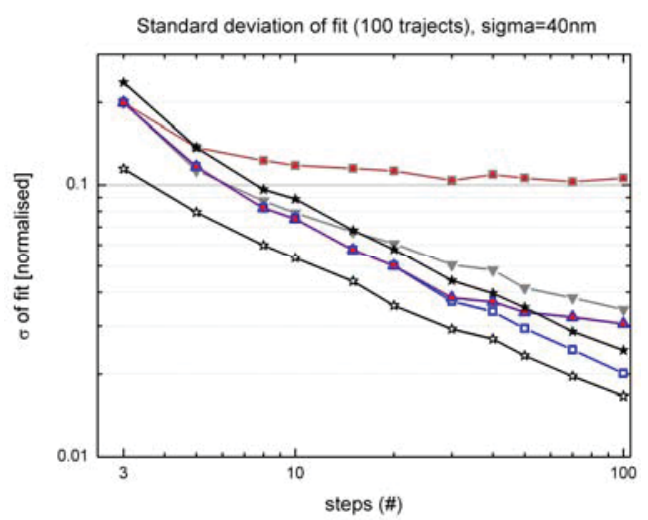

B

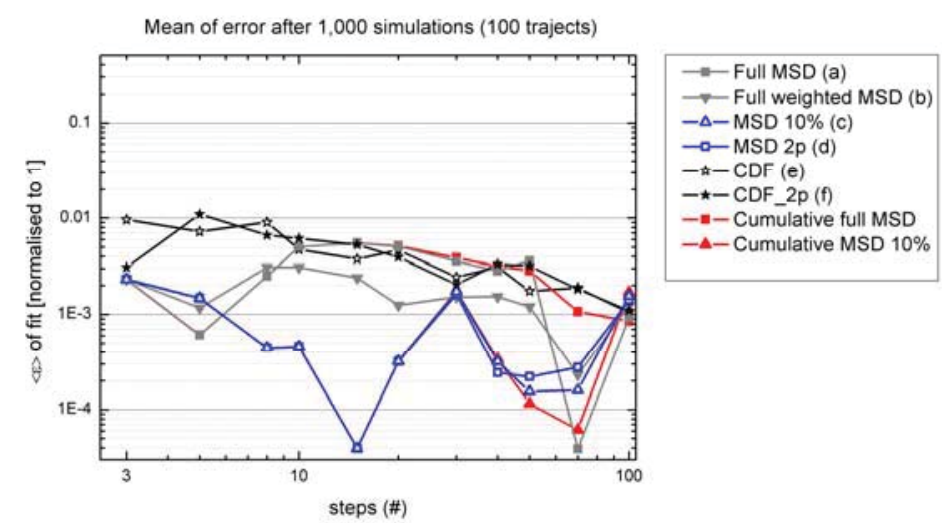

D

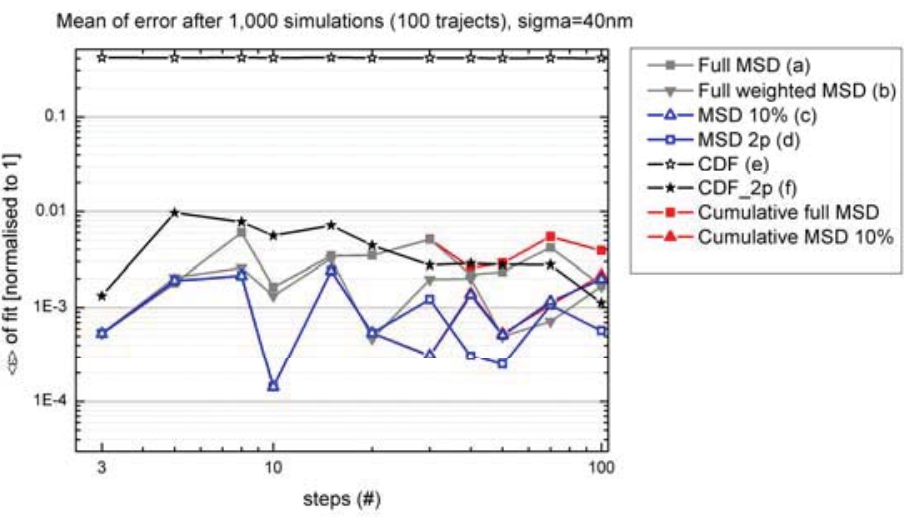

FIGURE 2.18 Error and standard deviation of MSD methods to obtain a one population diffusion coefficient. For 100 simulated trajectories exhibiting one-population Brownian motion ( $\left.D=0.1 \mu \mathrm{m}^{2} / \mathrm{s}, 25 \mathrm{fps}\right)$ of various lengths $(3,5,8, . ., 100$ steps $)$ as plotted on the $x$-axis, the diffusion value was determined from fitting the MSD curves of all trajectories. We added localization inaccuracy of $\sigma_{x y}=40 \mathrm{~nm}$ to the simulated trajectories (C,D). This fit was done using: the full curve (a), full curve weighted using the variance of each point (b), the first $10 \%$ (c), only the first two points (d), and using cumulative distribution function (CDF) fitting of steps (e), and using CDF of one-step and two-step distances (f). We repeated this 1,000 times, and looked at the standard deviation $\sigma(A, C)$, and the average mismatch $\langle\varepsilon\rangle(B, D)$ in the fitted diffusion values. The 1 step CDF method has the lowest standard deviation in the fitted values, but gives the wrong value when there is a localization inaccuracy as in practice. The most accurate way of using the points in the MSD curve is to only use the first two points of the MSD curve. 
Furthermore, it might seem, and it is often stated, that the CDF method is more accurate in determining the diffusion coefficient for a one population diffusion system compared to simply averaging the stepsizes as in MSD methods ${ }^{50}$, as it considers the whole distribution of stepsizes. In practice however, this is not always correct (Fig. 2.18). We used a simulation approach to determine the spread of diffusion coefficients found from both CDF and MSD methods.

We simulated one-population unconstrained diffusion for 100 trajectories of various lengths, with a relatively small localization error compared to the diffusion coefficient, so for $\beta=\sigma^{2} /(D \Delta t)$ ratio smaller than 1 , see ref. 25. We found that, for all lengths of trajectories, a CDF fit with only 1 stepsize is indeed, but only slightly, more accurate compared to the best MSD based fit; the value is of course wrong when not corrected for the added localization inaccuracy to the real diffusion coefficient. In practice this means we have to use the CDF of 2 steps too, and use the difference for CDF 2 steps and CDF 1 step to determine the diffusion coefficient. This 2 steps CDF methods has been described in detail in section 2.5.2. We found this last method however to be less accurate compared to the best MSD based fit where we take only the first two points in the curve (also the 1-steps and 2-steps). Using only the first two points in the MSD curve was the best MSD based fit for this ratio of $\beta$. Therefore the CDF will not be taken as a method for classification (in chapter 3), as the MSD is preferred for one population diffusion. Nevertheless, the CDF method has a known PDF for a distribution with multiple diffusion constants unlike the windowed MSD distribution, so this is still a straightforward method to find the global diffusion constant values and fractions when there are enough datapoints to build a reliable CDF.

\subsubsection{Relative confinement}

Inspired by the confinement detection method of $\operatorname{Simson}^{39}$, Meilhac used a slightly altered way to detect confinement ${ }^{41}$, which we shortly summarize here. The relative confinement is defined by the parameter $L$ as:

$$
\begin{gathered}
L\left(t_{0}+\frac{1}{2} \delta t\right)=\delta t / \operatorname{variance}(s) \\
s=\left|r(t)-r\left(t_{0}+\frac{1}{2} \delta t\right)\right| \text { on interval } t=\left[t_{0} . . t_{0}+\delta t\right]
\end{gathered}
$$

An illustration of the distances $s$ is given by arrows in Fig. 2.17A.

\subsubsection{Radius of gyration evolution}

The use of the radius of gyration has been first proposed by Saxton to measure asymmetry in single molecule trajectories ${ }^{37}$, and it was demonstrated by Elliott e.a. that it could also be used to detect confinement ${ }^{42}$. The gyration radius is a measure of the space that is explored (defined by radius $R_{g}$ ) by the molecule within the segment, hence the radius will have a lower value for slow diffusion than for fast diffusion. Therefore the gyration radius is a local measure of the diffusion of a molecule, and can be used as a differentiation criterion in classification.

The gyration radius $R_{g}$ is defined as:

$$
R_{g}=\sqrt{R_{1}^{2}+R_{2}^{2}}
$$

where $R_{1}$ and $R_{2}$ are the eigenvalues of the gyration tensor $\boldsymbol{T}$ : 


$$
\boldsymbol{T}=\left(\begin{array}{cc}
\frac{1}{N} \sum_{i=1}^{N}\left(x_{i}-\langle x\rangle\right)^{2} & \frac{1}{N} \sum_{i=1}^{N}\left(x_{i}-\langle x\rangle\right)\left(y_{i}-\langle y\rangle\right) \\
\frac{1}{N} \sum_{i=1}^{N}\left(x_{i}-\langle x\rangle\right)\left(y_{i}-\langle y\rangle\right) & \frac{1}{N} \sum_{i=1}^{N}\left(y_{i}-\langle y\rangle\right)^{2}
\end{array}\right)
$$

with $i$ enumerating all subsequent positions $\left(x_{i}, y_{i}\right)$ in a segment of length $N$.

\subsubsection{Super-resolution reconstructions}

Although this has not been mentioned specifically in literature before, equivalents to superresolution STORM and PALM images $^{51,52}$ can be made with the localizations by running a STORM analysis on the localized positions in time from single-molecule tracking data. According to the ergodic principle, it should not make a difference whether a recording is taken from a subset of moving molecules or from a more complete set of fixed (immobilized) molecules. Indeed, the resulting reconstruction images (Fig. 2.19) look very much like STORM images of the same receptor protein ${ }^{53}$. Yet it is very difficult to find biologically interesting insights from such images. In the next chapter we will discuss an extension to using mobility information together with these super-resolution reconstruction images.
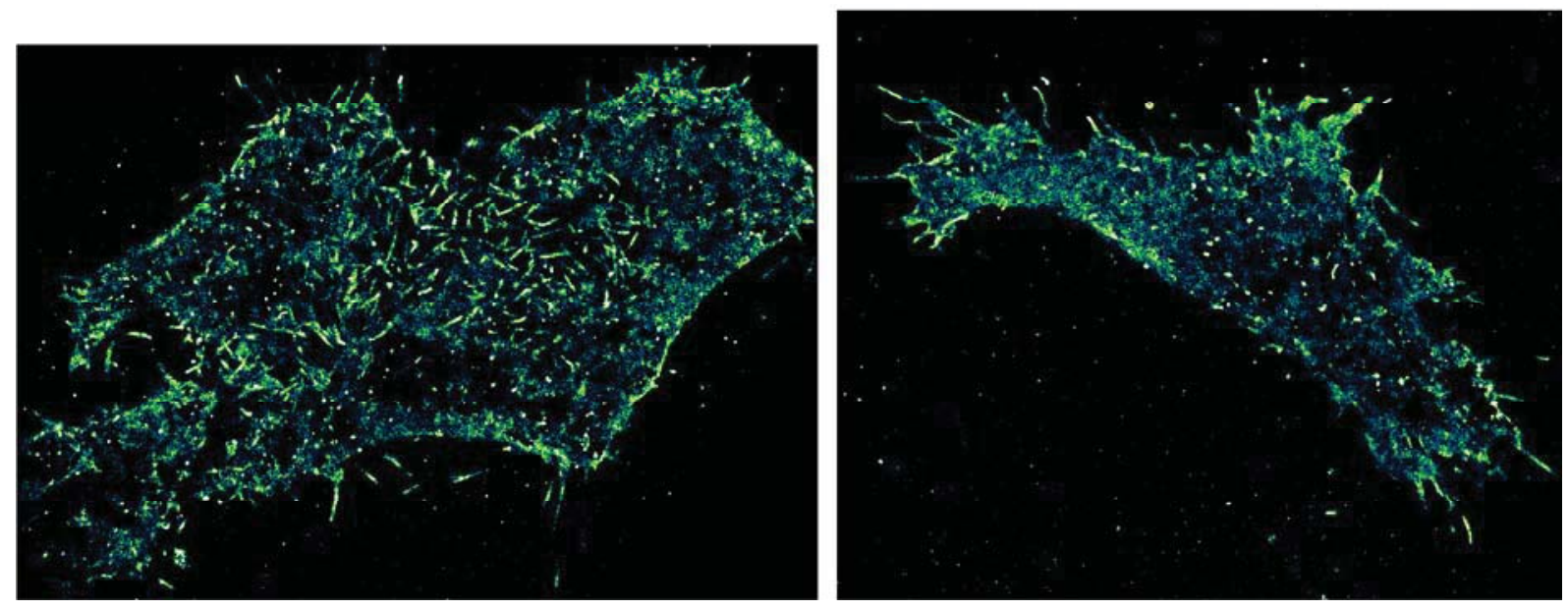

FIGURE 2.19 Images of the positions of EGF receptors in two MCF7 cells during 30 seconds reconstructed at high resolution. Single-molecule tracking data can be processed using STORM analysis to reconstruct a high resolution image of a protein of interest. Experimental details are discussed in later chapters.

\subsection{Discussion and conclusion}

We have designed and built a triple-colour $(473 \mathrm{~nm}, 532 \mathrm{~nm}, 647 \mathrm{~nm})$ single molecule sensitive total-internal reflection fluorescence (TIRF) microscope optimized for single-molecule tracking experiments. The design was optimized to yield a high detection efficiency, record with short illumination time and at high precision, while being able to make use of standard-sized filters and dichroic mirrors. The setup was equipped with a stage heating plate and objective heater to allow measurements at physiological temperatures $\left(33-37.5^{\circ} \mathrm{C}\right.$ within the sample between 5 and 30 minutes).

A unique aspect of our design is the use of high-power solid-state lasers with high-frequency modulation possibility that can be used to get stroboscopic illumination. This reduces the signal blur caused by motion of the fluorescent probe during the acquisition time, which has a positive effect on the correct readout of mobility information; this is because the value of the diffusion 
coefficient is underestimated due to the averaging blur during exposure ${ }^{40,54}$. Thanks to the highpower of the lasers, we can record 100 photons from single molecules within 2 milliseconds over a homogeneous field of view with a diameter around $60 \mu \mathrm{m}$ (500 pixels) at video-rate (24-30 fps). By making use of a rotating diffuser, we could maximize the amount of light used for a homogenous illumination pattern, while simultaneously reducing interference effects without the need to use fiber optics.

Another unique aspect in our microscope is the free-space implementation of the lasers as illumination light sources. This implementation makes it more practical to achieve a high coupling efficiency of laser light into the microscope, which reduces the minimum illumination time or the need to purchase lasers of higher powers. Once aligned, the setup can be used on other days without any needs for readjustments to the alignment. To change from widefield to TIRF requires only the flipping of a small two-lens system, where the excitation angle of the beam can be changed easily by a knob of a micro-stage, which changes the lateral position on one of the lenses. This makes it user-friendly to switch from and to TIRF illumination while browsing a sample, for instance to find a cell with low autofluorescence.

Furthermore, the performance characteristics obtained can be considered state-of-the-art, and are similar or better than in other systems described ${ }^{18,55}$. For example, we achieved an optical localization precision $\sigma_{\mathrm{xy}}$ of $20 \mathrm{~nm}$ for organic fluorophores, excited at 3.1 and $1.7 \mathrm{~kW} / \mathrm{cm}^{2}$ during 9 and $2 \mathrm{~ms}$ for the green and red excitation intensity respectively. Finally, we used heating equipment which may have negative influence on the drift of a setup. By optimizing the attachment of the heating plate to the stage of the microscope, we could minimize this drift to peak values of $85 \mathrm{~nm}$ during the time of a measurement.

We also described the software to obtain the protein trajectories from the microscopy recordings. This software is based on the latest mathematical insights in tracking fluorescent molecules in cells, and can analyse a typical 1,000 frames $512 \times 512$ pixel image series in about 5 minutes time on our computer thanks to the execution of the Gaussian fitting routine on the GPU card. We described the settings used in this software for the tracking of typical measurements. Several fixes to the software were proposed, and two extensions for the software were developed to make the processing of microscopy recordings easier and more user-friendly.

In this chapter we have also summarized analysis methods currently used to characterize the motion of proteins from their trajectories. We have discussed the limitations of these methods, and have given our opinion on their best use in single-molecule tracking data. Due to the dynamic nature of the motion of proteins, we emphasize that local methods are needed to obtain nonaveraged information on the type of motion exhibited by proteins. A model for the diffusion system behind protein motion might be best described as a system with multiple populations of purely diffusive motion. These populations can be robustly obtained by a cumulative (probability) density function (CDF) fit of all squared step sizes within the protein trajectories recorded.

Finally, we would like to give two recommendations to take into account when further developing the setup described. The current design made use of the bottom port of the microscope, however this requires very precise placements of 1 inch components in the illumination path. It will be much easier when the back port of the microscope is used for illumination or when 2 inch optics are used. Concerning the detection part of the scheme, the field of view can be slightly larger when the dichroic mirror, used to separate the illumination and emission light, and the notch filters (D2, N1 and N2 in Fig. 2.2) are larger than the standard-sized filters (25x36mm for mirrors, and $25 \mathrm{~mm}$ diameter for filters), or would be placed at a smaller distance from the objective, since the standard-sized filters block a small part of the outer regions of the emission light that would otherwise have been detectable by the cameras. Normally a tube lens is used within a reasonably small distance from the objective to prevent beam divergence and collect all the emission light. We had however removed this tube lens to increase the detection efficiency. Secondly, the circular diffuser used provided a more homogenous illumination pattern 
than a Gaussian shaped laser beam or a Gaussian diffuser. It should also be possible to take a diffuser with a square pattern, which better fits the field of view of the camera. However we have not tested this as we found the circular pattern suitable for our application of imaging single cells, which are usually of oval shape. 


\subsection{References}

1. Holtzer, L. \& Schmidt, T. in Single Particle Tracking and Single Molecule Energy Transfer (Bräuchle, C. Lamb, D. C. \& Michaelis, J.) (WILEY-VCH Verlag $\mathrm{GmbH}, 2010$ ).

2. De Keijzer, S. et al. A spatially restricted increase in receptor mobility is involved in directional sensing during Dictyostelium discoideum chemotaxis. J. Cell Sci. 121, 1750-7 (2008).

3. Sergé, A., Bertaux, N., Rigneault, H. \& Marguet, D. Dynamic multiple-target tracing to probe spatiotemporal cartography of cell membranes. Nat Methods 5, 687-94 (2008).

4. Low-Nam, S. T. et al. ErbB1 dimerization is promoted by domain co-confinement and stabilized by ligand binding. Nat. Struct. Mol. Biol. 18, 1244-1249 (2011).

5. Sako, Y., Minoghchi, S. \& Yanagida, T. Singlemolecule imaging of EGFR signalling on the surface of living cells. Nat. Cell Biol. 2, 168-72 (2000).

6. Long, F., Zeng, S. \& Huang, Z.-L. Localization-based super-resolution microscopy with an sCMOS camera Part II: Experimental methodology for comparing sCMOS with EMCCD cameras. Opt. Express 20, 17741 (2012).

7. Wieser, S. \& Schütz, G. J. Tracking single molecules in the live cell plasma membrane-Do's and Don't's. Methods 46, 131-40 (2008).

8. Wieser, S., Moertelmaier, M., Fuertbauer, E., Stockinger, H. \& Schütz, G. J. (Un)confined diffusion of CD59 in the plasma membrane determined by high-resolution single molecule microscopy. Biophys. J. 92, 3719-28 (2007).

9. Hamamatsu. Datasheet ORCA Flash4. at <http://hamamatsucameras.com/flash4/index.php>

10. Dempsey, G. T., Vaughan, J. C., Chen, K. H., Bates, M. \& Zhuang, X. Evaluation of fluorophores for optimal performance in localization-based superresolution imaging. Nat. Methods 8, 1027-36 (2011).

11. Andor. Andor iXon 897 Specifications. at <http://www.andor.com/pdfs/specifications/Andor_iXo n_897_Specifications.pdf>

12. Ober, R. J., Ram, S. \& Ward, E. S. Localization accuracy in single-molecule microscopy. Biophys. J. 86, 1185-200 (2004).

13. Harms, G. S., Cognet, L., Lommerse, P. H., Blab, G. A. \& Schmidt, T. Autofluorescent proteins in singlemolecule research: applications to live cell imaging microscopy. Biophys. J. 80, 2396-408 (2001).

14. Molecular Probes Handbook, A Guide to Fluorescent Probes and Labeling Technologies, 11th Edition. at $<$ http://www.invitrogen.com/site/us/en/home/Referenc es/Molecular-Probes-The-Handbook.html>

15. Ram, S., Ward, E. S. \& Ober, R. J. Beyond Rayleigh's criterion: a resolution measure with application to single-molecule microscopy. Proc. Natl. Acad. Sci. U. S. A. 103, 4457-62 (2006).

16. Stallinga, S. \& Rieger, B. Accuracy of the gaussian point spread function model in 2D localization microscopy. Opt. Express 18, 24461-76 (2010).

17. Pixel Response Effects on CCD Camera Gain Calibration. at <http://www.mirametrics.com/tech note ccdgain.htm>

18. Bakker, G. J. LFA-1 dynamics on the membrane of leukocytes : a single dye tracing study, $\mathrm{PhD}$ thesis, University of Twente. (2011)

19. Smith, C. S., Joseph, N., Rieger, B. \& Lidke, K. A. Fast, single-molecule localization that achieves theoretically minimum uncertainty. Nat. Methods 7 , 373-5 (2010)

20. Jaqaman, K. et al. Robust single-particle tracking in live-cell time-lapse sequences. Nat. Methods 5, 695702 (2008).

21. Sako, Y. \& Yanagida, T. Single-molecule visualization in cell biology. Nat. Rev. Mol. Cell Biol. Suppl, SS1-5 (2003).

22. Martin-Fernandez, M. L. \& Clarke, D. T. Single Molecule Fluorescence Detection and Tracking in Mammalian Cells: The State-of-the-Art and Future Perspectives. Int. J. Mol. Sci. 13, 14742-65 (2012).

23. Kusumi, a, Sako, Y. \& Yamamoto, M. Confined lateral diffusion of membrane receptors as studied by single particle tracking (nanovid microscopy). Effects of calcium-induced differentiation in cultured epithelial cells. Biophys. J. 65, 2021-40 (1993).

24. Saxton, M. J. \& Jacobson, K. Single-particle tracking applications to membrane dynamics. Annu. Rev. Biophys. Biomol. Struct. 26, 373-99 (1997).

25. Michalet, X. Mean square displacement analysis of single-particle trajectories with localization error: Brownian motion in an isotropic medium. Phys. Rev. E 82, 041914 (2010)

26. Engelman, D. M. Membranes are more mosaic than fluid. Nature 438, 578-80 (2005).

27. Shaw, A. S. Lipid rafts: now you see them, now you don't. Nat. Immunol. 7, 1139-42 (2006).

28. Jacobson, K., Mouritsen, O. G. \& Anderson, R. G. W. Lipid rafts: at a crossroad between cell biology and physics. Nat. Cell Biol. 9, 7-14 (2007).

29. Dietrich, C., Yang, B., Fujiwara, T., Kusumi, A. \& Jacobson, K. Relationship of lipid rafts to transient confinement zones detected by single particle tracking. Biophys. J. 82, 274-84 (2002).

30. Schütz, G. J., Schindler, H. \& Schmidt, T. Singlemolecule microscopy on model membranes reveals anomalous diffusion. Biophys. J. 73, 1073-80 (1997)

31. Helmuth, J. a, Burckhardt, C. J., Koumoutsakos, P., Greber, U. F. \& Sbalzarini, I. F. A novel supervised trajectory segmentation algorithm identifies distinct types of human adenovirus motion in host cells. $J$. Struct. Biol. 159, 347-58 (2007).

32. Wieser, S., Axmann, M. \& Schütz, G. J. Versatile analysis of single-molecule tracking data by comprehensive testing against Monte Carlo simulations. Biophys. J. 95, 5988-6001 (2008)

33. Xiao, Z., Zhang, W., Yang, Y., Xu, L. \& Fang, X. Single-molecule diffusion study of activated EGFR implicates its endocytic pathway. Biochem. Biophys. Res. Commun. 369, 730-4 (2008).

34. Rong, G. \& Reinhard, B. M. Monitoring the size and lateral dynamics of ErbB1 enriched membrane domains through live cell plasmon coupling microscopy. PLoS One 7, e34175 (2012).

35. Orr, G. et al. Cholesterol dictates the freedom of EGF receptors and HER2 in the plane of the membrane. Biophys. J. 89, 1362-73 (2005).

36. Manley, S. et al. High-density mapping of singlemolecule trajectories with photoactivated localization microscopy. Nat. Methods 5, 155-157 (2008).

37. Saxton, M. J. Lateral diffusion in an archipelago. Single-particle diffusion. Biophys. J. 64, 1766-80 (1993). 
38. Kusumi, A. et al. Paradigm shift of the plasma membrane concept from the two-dimensional continuum fluid to the partitioned fluid: high-speed single-molecule tracking of membrane molecules. Annu. Rev. Biophys. Biomol. Struct. 34, 351-78 (2005).

39. Simson, R., Sheets, E. D. \& Jacobson, K. Detection of temporary lateral confinement of membrane proteins using single-particle tracking analysis. Biophys. J. 69, 989-93 (1995).

40. Goulian, M. \& Simon, S. M. Tracking single proteins within cells. Biophys. J. 79, 2188-98 (2000).

41. Meilhac, N., Le Guyader, L., Salomé, L. \& Destainville, N. Detection of confinement and jumps in single-molecule membrane trajectories. Phys. Rev. E 73, 011915 (2006).

42. Elliott, L. C. C., Barhoum, M., Harris, J. M. \& Bohn, P.W. Trajectory analysis of single molecules exhibiting non-brownian motion. Phys. Chem. Chem. Phys. 13, 4326-34 (2011).

43. Semrau, S. \& Schmidt, T. Particle image correlation spectroscopy (PICS): retrieving nanometer-scale correlations from high-density single-molecule position data. Biophys. J. 92, 613-21 (2007).

44. Semrau, S., Holtzer, L., González-Gaitán, M. \& Schmidt, T. Quantification of Biological Interactions with Particle Image Cross-Correlation Spectroscopy (PICCS). Biophys. J. 100, 1810-8 (2011).

45. Moerters, P. \& Peres, Y. Brownian Motion. 416 (Cambridge University Press, 2010).
46. Brameshuber, M. \& Schütz, G. J. in Springer Series on Fluorescence 1-37 (Springer Berlin Heidelberg, 2012). doi:10.1007/4243_2011_38

47. Chandrasekhar, S. Stochastic problems in physics and astronomy. Rev. Mod. Phys. 15, 1-89 (1943).

48. Qian, H., Sheetz, M. P. \& Elson, E. L. Single particle tracking. Analysis of diffusion and flow in twodimensional systems. Biophys. J. 60, 910-21 (1991).

49. Saxton, M. J. Single-particle tracking: the distribution of diffusion coefficients. Biophys. J. 72, 1744-53 (1997).

50. Brauchle, C., Lamb, D. C. \& Michaelis, J. Single Particle Tracking and Single Molecule Energy Transfer. (WILEY-VCH Verlag $\mathrm{GmbH}$ ).

51. RustMJ, B. Imaging by stochastic optical reconstruction microscopy (STORM). Nat. Methods 3, 793 (2006).

52. Betzig, E. et al. Imaging intracellular fluorescent proteins at nanometer resolution. Science 313, 16425 (2006).

53. Winckler, P. et al. Identification and super-resolution imaging of ligand-activated receptor dimers in live cells. Sci. Rep. 3, 2387 (2013).

54. Berglund, A. Statistics of camera-based singleparticle tracking. Phys. Rev. E 82, 011917 (2010).

55. Elliott, L. C. C. Single molecule tracking studies of lower critical solution temperature transition behavior in poly( $\mathrm{N}$-isopropylacrylamide), $\mathrm{PhD}$ thesis, University of Illinois. (2011). 


\section{Appendix}

TABLE 2.3 Settings used in the tracking software for the tracking of the recordings in this thesis. A comment is given for each setting for clarification.

\begin{tabular}{|c|c|c|}
\hline Parameter & Value used & Comment \\
\hline Dat.PixelSize & 0.119 & Calibrated $\mu \mathrm{m} /$ frame \\
\hline Dat.TimeStep & 0.04468 & Inverse frame rate \\
\hline Dat.ch_bin & [1] & $\begin{array}{l}\text { Our image recordings contains only } 1 \text { channel } \\
\text { per file. The distinct channels are recorded } \\
\text { and saved as distinct files, and tracking is } \\
\text { performed on each file individually. }\end{array}$ \\
\hline Params.psf & {$\left[\begin{array}{llll}0.84 & 0.84\end{array}\right]$} & $\begin{array}{l}\text { Point Spread Function (PSF) radius for both } \\
\text { dimensions. For the images of red-excitable } \\
\text { fluorescence, } 0.99 \text { was used. }\end{array}$ \\
\hline Params.CCDGain & 32.5 & $\begin{array}{l}\text { For the files from the camera recording green- } \\
\text { excitable dyes. For the other camera } 49.9 \text { was } \\
\text { used here. }\end{array}$ \\
\hline Params.CCDOffset & 0 & $\begin{array}{l}\text { Since we pre-filter our images to subtract } \\
\text { baselines, the constant CCD offset is already } \\
\text { subtracted. }\end{array}$ \\
\hline Params.Intensity & 3.7 & $\begin{array}{l}\text { Values between } 2.5 \text { and } 4.0 \text { were used here, } \\
\text { depending on the standard deviance of the } \\
\text { images. We roughly chose between } 1 \text { and } 2 \\
\text { times the standard deviance of the images. }\end{array}$ \\
\hline Params.FitBoxSize & 7 & $\begin{array}{l}\text { The PSF in our images expanded not further } \\
\text { than a } 7 \times 7 \text { pixel subimage. }\end{array}$ \\
\hline Params.Iterations & 10 & \\
\hline Params.MaxCudaFits & 30000 & $\begin{array}{l}\text { The default setting gave errors on our GPU } \\
\text { cards. }\end{array}$ \\
\hline Params.MinCRLBSigma & 0.5 & $\begin{array}{l}\text { This is actually the maximum allowed Cramer } \\
\text { Rao Lower Bound value, which could be } \\
\text { regarded to be the localization error. When } \\
\text { the localization was so poor that it was larger } \\
\text { than } 0.5 \text { pixel ( } 60 \mathrm{~nm}), \text { we did not want to } \\
\text { include the position to avoid spurious signals. }\end{array}$ \\
\hline Params.MinPValue & 0.01 & $\begin{array}{l}\text { We chose to only use the CRLB and Photon } \\
\text { count as threshold to include signals, and not } \\
\text { the } p \text {-value of the MLE fit. Therefore a value } \\
\text { was chose that included all signals that passed } \\
\text { the other thresholds. }\end{array}$ \\
\hline Params.MinPhotons & 10 & $\begin{array}{l}\text { We found this to be a good compromise } \\
\text { between still detecting weak signals but } \\
\text { prevent spurious signals to be included. }\end{array}$ \\
\hline Params.ConnectParams. & Value used & Comment \\
\hline costMatF2Fparams.D & [0.189 0.189] & $\begin{array}{l}\text { [0.06*Dat.TimeStep/Dat.PixelSize^2 } \\
0.06 * \text { Dat.TimeStep/Dat.PixelSize } 2 \text { ] } \\
\mathrm{D}=0.06 \mu \mathrm{m}^{2} / \mathrm{s} \text { converted to pixeF2/frame. } \\
\text { We chose } 0.06 \text { as we had to choose a one- } \\
\text { population diffusion constant, this value is in }\end{array}$ \\
\hline
\end{tabular}




\begin{tabular}{|c|c|c|}
\hline & & $\begin{array}{l}\text { between the diffusion constants of the two } \\
\text { diffusion population found for the receptors } \\
\text { studied, which therefore provides a likely step } \\
\text { size distribution of the two-population system. }\end{array}$ \\
\hline $\begin{array}{l}\text { costMatF2Fparams.maxSearchDis } \\
\mathrm{t}\end{array}$ & [4 4] & $\begin{array}{l}\text { Since a maximum search distance } L \text { of } 2.55 \\
{ }^{*} \text { sqrt }\left(4^{*} D^{*} \tau_{\text {lag }}\right) \text { includes } 99 \% \text { of step sizes, } \\
\text { with } D=0.15 \mu \mathrm{m}^{2} / \mathrm{s} \text { for the fastest state of the } \\
\text { receptors studied, this distance equals } 3.51 \\
\text { pixels. }\end{array}$ \\
\hline costMatF2Fparams.kon & 0.1 & \\
\hline costMatF2Fparams.koff & 0.0001 & $\begin{array}{l}\text { We experienced several linking problems at } \\
\text { first, for example spurious stops of a trajectory } \\
\text { together with an immediate start of a new } \\
\text { trajectory, while the new trajectory was } \\
\text { clearly a continuation of the existing } \\
\text { trajectory. We found that we need to push the } \\
\text { software towards continuing existing } \\
\text { trajectories, by finding it very unlikely that a } \\
\text { signal would disappear. In fact, when a signal } \\
\text { truly stops, it would not be detected in the } \\
\text { first place, so the trajectory will then end } \\
\text { anyway. Therefore the } k_{\text {on }} \text { rate was chosen to } \\
\text { be a realistic } 0.1 \text { (from a blinking state), while } \\
\text { the } \text { kff }_{\text {off }} \text { was set at an unrealistic low number to } \\
\text { give preference to a continuation of a } \\
\text { trajectory. }\end{array}$ \\
\hline costMatGCparams.timeWindow & 10 & $\begin{array}{l}\text { This is the maximum number of frames to } \\
\text { allow a signal to be continued to be tracked, in } \\
\text { other words, the maximum allowed blinking } \\
\text { time. We found that } 0.5 \text { second ( } 12 \text { frames) } \\
\text { was too long, in that case clear spurious } \\
\text { reconnections started to occur. }\end{array}$ \\
\hline costMatGCparams.D & {$\left[\begin{array}{lll}0.01 & 0.01\end{array}\right]$} & $\begin{array}{l}\text { For reconnections, we prefer to allow the } \\
\text { molecule to diffuse at a slow speed over } \\
\text { molecules that diffuse faster. }\end{array}$ \\
\hline maxSearchDistPerFrame & {$\left[\begin{array}{ll}3 & 3\end{array}\right]$} & $\begin{array}{l}\text { We however allow up to (almost) the normal } \\
\text { maximum search distance for reconnection. } \\
\text { Large distance reconnection will only occur } \\
\text { when no other signals are observed closer by. }\end{array}$ \\
\hline maxSearchDist & [10 10] & $\begin{array}{l}\text { At the density of molecules in our images, this } \\
\text { is a realistic distance. }\end{array}$ \\
\hline minTrackLen & 2 & $\begin{array}{l}\text { Signals that are detected as a molecule for } \\
\text { only } 1 \text { frame are very likely spurious signals. }\end{array}$ \\
\hline costMatGCparams.kon & 0.1 & As detailed before. \\
\hline costMatGCparams.koff & 0.0001 & \\
\hline Params.TrackFunction & 'obj.makeTrack & $\begin{array}{l}\text { An update in the software which allows to run } \\
\text { the linking part using a compiled C++ } \\
\text { executable. }\end{array}$ \\
\hline
\end{tabular}





\title{
Chapter 3
}

\section{Classification of dynamical diffusion states in single molecule tracking microscopy}

\author{
Peter J. Bosch ${ }^{1}$ \\ Johannes S. Kanger ${ }^{2}$ \\ Vinod Subramaniam ${ }^{1,2,3}$
}

1. Nanobiophysics, MESA+ Institute for Nanotechnology and MIRA Institute for Biomedical Technology and Technical Medicine, University of Twente, PO Box 217, 7500AE Enschede, The Netherlands

2. MIRA Institute for Biomedical Technology and Technical Medicine, University of Twente, The Netherlands

3. Present address: FOM Institute AMOLF, Science Park 104, 1098 XG Amsterdam, The Netherlands 


\begin{abstract}
Single-molecule tracking of membrane proteins by fluorescence microscopy is a promising method to investigate dynamic processes in live cells. Translating the trajectories of proteins to biological implications, such as protein interactions, requires the classification of protein motion within the trajectories. Spatial information of protein motion may reveal where the protein interacts with cellular structures, since binding of proteins to such structures often alters their diffusion speed. For dynamic diffusion systems, we provide an analytical framework to determine in which diffusion state a molecule is residing during the course of its trajectory. We compare different methods for the quantification of motion to utilize within this framework for the classification of two diffusion states (two populations with different diffusion speed). We found that a gyration quantification method and a Bayesian statistics based method are the most accurate in diffusion state classification for realistic experimentally obtained datasets, of which the gyration method is much less computationally demanding. After classification of the diffusion, the lifetime of the states can be determined, and images of the diffusion states can be reconstructed at high resolution. Simulations validate these applications. We apply the classification and its applications to experimental data to demonstrate the potential of this approach to obtain further insights into the dynamics of cell membrane proteins.
\end{abstract}




\subsection{Introduction}

It remains an elusive dream to be able to follow a protein and its interactions as the protein travels through the cell during its lifespan. Nevertheless, single molecule tracking by fluorescence microscopy allows one to follow a protein in a living cell at high resolution for a short period of time and record its trajectory ${ }^{1-6}$. Tracking of proteins in live cells is a unique approach to obtain details on dynamical protein association and dissociation kinetics in a spatiotemporal manner, and complements other fluorescence microscopy techniques ${ }^{7-9}$. Single molecule tracking techniques have given us valuable insight in the dynamics and biological functions of proteins $s^{6,10-}$ 12 and the organization of the plasma membrane ${ }^{13-16}$. Despite methodological advances and the insight obtained by current analysis methods, there remains a need to further develop analysis tools that can translate experimental data into biological insights. For example, spatiotemporal information on the diffusion of membrane proteins would contribute to a biophysical understanding of the organization of these protein complexes.

Trajectories of proteins obtained by tracking techniques contain information about the interaction and functional state of the protein. For example, the phosphorylation state of many membrane bound tyrosine kinase receptors is related to their formation of dimers or higher order aggregates ${ }^{17,18}$. Clearly, proteins associated with these aggregates are expected to show lower mobility than free monomeric receptor molecules, which is reflected in their trajectories. Additionally, proteins often transiently interact with other molecules in nanoscale compartmentalization structures in the plasma membrane or with cytoskeletal structures, both resulting in transient slowed diffusion or confinement ${ }^{4-6,19-23}$. Not only do interactions with molecules alter protein mobility, but the mobility of a protein also affects the possibility of interactions with other molecules ${ }^{24-26}$. A detailed knowledge of the interactions of proteins and their dynamics is therefore important to understand the underlying signal transduction processes and to model the cellular signal regulatory system ${ }^{24-28}$.

Translating the trajectories of proteins to biological events, such as protein interactions, requires the classification of protein motion within the trajectories. Protein species transiently exhibit different types of motion. The motion of membrane proteins can often be described by two dynamic populations of pure Brownian diffusion ${ }^{6,19,23}$, which we refer to as the diffusion states (Fig. 3.1A). It is however nontrivial to accurately determine in which diffusion state the protein is residing during the measured trajectory. Several issues hamper faultless state classification. Proteins exhibiting different diffusion states often have overlapping distributions of step sizes (Fig. 3.1B). Furthermore, the localization of proteins has a limited accuracy, and the switching between the diffusion states is a stochastic process. Diffusion state classification methods are needed to determine when, and in what regions, the protein exhibited distinct diffusion behavior. These regions might point towards a role of certain cellular structures in the function of the studied protein species. Also the lifetimes of these diffusion states (the inverse kinetic rate) can be directly derived from the diffusion state durations, and are useful parameters to comprehend the role of the studied protein in complexes associated with cellular regulatory systems. The combined insight may eventually reveal the spatiotemporal design principles of cell decision-making ${ }^{27}$.

A widely used analysis method for single molecule tracking data considers complete trajectories using mean squared displacement (MSD) curves $^{3,29-32}$. For homogenous motion, the shape of the MSD curve contains information about the nature of the diffusion, e.g. pure, confined, or hop diffusion ${ }^{3,13,33,34}$. Since the MSD curve is composed of averages of all distances, transient diffusion states cannot be resolved by these full-trajectory MSD analysis (Fig. S1 in the Supporting Material). When it was realized that protein motion is not homogeneous, but show transient effects $^{1,4,10,22,35}$, local methods were developed that considered subtrajectories (segments) of a trajectory ${ }^{4,34,36,37}$. These methods are however hampered by the limited number of positions within one segment to obtain accurate diffusion coefficients or confinement strengths. An alternative 
Monte Carlo based method ${ }^{38}$ is particularly useful to find the kinetic rates between welldifferentiated diffusion populations. This method finds diffusion coefficients, their fractions, and the switching rates for the whole set of trajectories, but does not spatially resolve the states. Therefore, we propose a new approach which uses a global method (analyzing all trajectories obtained) to determine the different diffusion states of the protein studied, whereas local methods are used to classify short segments of a trajectory to one of the diffusion states found. We compare several local methods to classify parts of trajectories (segments) to a diffusion state.
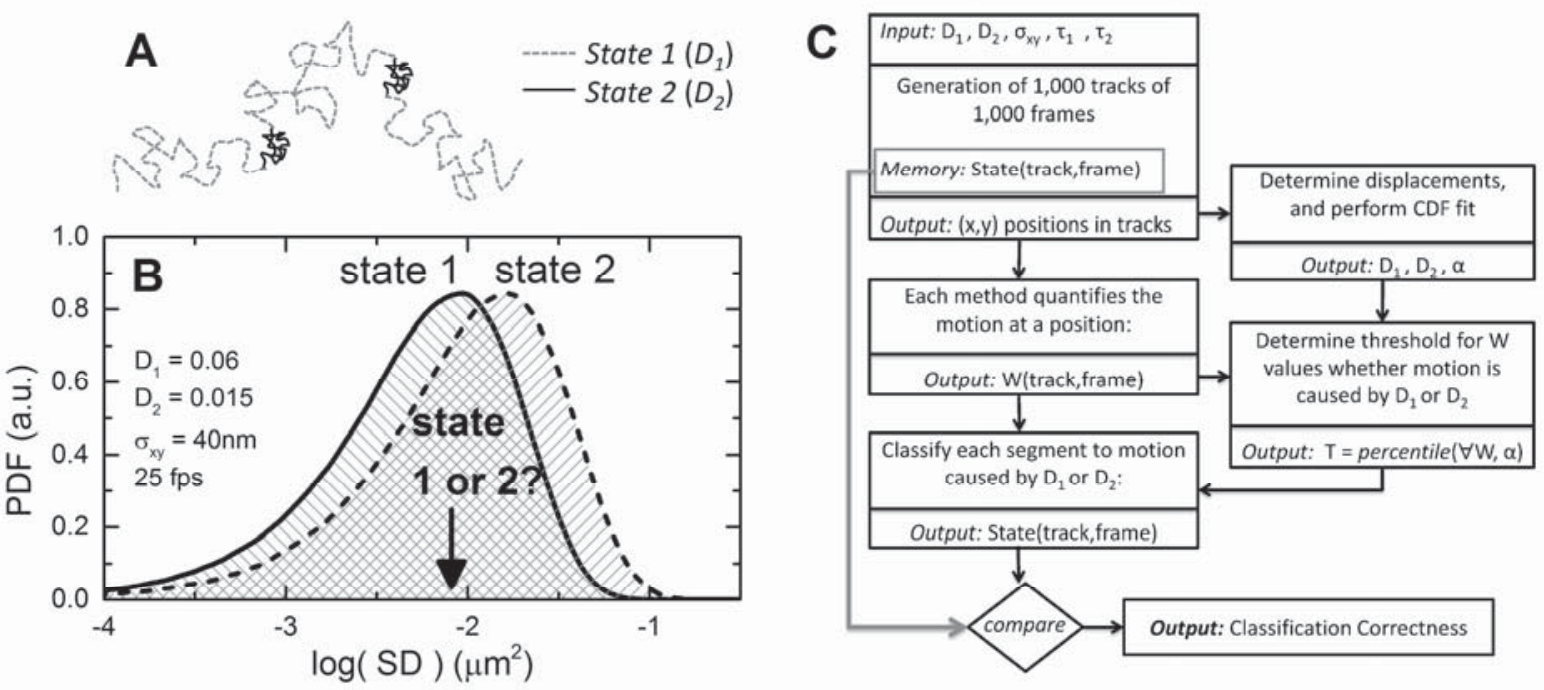

FIGURE 3.1 Proposed framework for state classification and problem statement. $(A)$ Schematic to illustrate a typical trajectory of a single protein on a plasma cell membrane, displaying switching behavior between two states with different diffusion coefficients. $(B)$ Distributions of observed squared displacements (SD) resulting from different diffusion coefficients show large overlap. A measured step size value (an example is indicated with an arrow) cannot be classified with high certainty to unambiguously originate from a particular state, which demonstrates one of the problems to be solved for diffusion state classification. To compose this histogram, a localization inaccuracy $\sigma_{x y}$ of $40 \mathrm{~nm}$ was added to the positions in the simulations. $(C)$ Scheme of the methodology followed to test the various classification methods on correctness of state classification. After generation of simulated trajectories with dynamic state allocation, we determine the diffusion constants $\left(D_{1}\right.$ and $\left.D_{2}\right)$ and the fraction $(\alpha)$ of the fast state from all displacements using a CDF fit. The track is divided in segments of a certain window length $(\mathrm{N})$, and for each segment the tested quantification methods provide a value $\mathrm{W}$ using only the positions in that segment. For each segment the motion is classified as fast or slow diffusion. The threshold $(T)$ for classification is determined from all values $W$ and the fraction $\alpha$. The center position of the segment is classified as slow diffusion when $\mathrm{W}$ is smaller than the threshold $\mathrm{T}$, and as fast diffusion otherwise. The found state is compared with the actual (remembered) state to yield the classification correctness. The same scheme is followed for the diffusion state classification of experimental data. Although the correctness clearly cannot be determined in that case, an estimation of the correctness can be determined by performing simulations at the parameters found by the CDF fit.

\subsubsection{Proposed scheme for diffusion state classification}

For pure diffusion systems, the multiple diffusion states can be accurately determined using a fit of the cumulative distribution function (CDF) of the squared displacements ${ }^{20}$. In this chapter we assume that the motion of membrane proteins can be described by two states of Brownian diffusion, termed the fast and the slow population. Whether this assumption is correct can be checked beforehand by looking at the residuals of a fit of the cumulative distribution function (CDF) of step sizes in two-population diffusion (detailed later). After obtaining accurate diffusion parameters by this fit, local methods are used only to classify short segments of a trajectory to one 
of the diffusion states found. Existing local diffusion or confinement detection methods ${ }^{34,36,37} \mathrm{can}$ be expanded to yield a local quantification measure which can be used for classification. Subsequently, the classification to a diffusion state is based on a threshold for the quantification measures. This threshold is objectively set using the parameters describing the diffusion states (determined by the fit), and the threshold is therefore based on the experimental data. Thereby we have eliminated the subjective manual thresholding of earlier confinement methods to detect transitions between motion states. The need for manual thresholding was earlier mentioned as a disadvantage of using window (segments) based methods ${ }^{39}$.

We emphasize that there is no need to determine local diffusion values of segments, since the CDF fit has already accurately provided the diffusion values present within the trajectories. The segments only need to be classified to one of the diffusion states. The length of the segments should be carefully chosen such that the corresponding duration is shorter than the typical switching time between states, whereas the duration must be long enough to obtain an accurate measure for the classification. We test different local methods and the influence of different segment lengths for diffusion classification using two state Brownian dynamics simulations (Fig. 3.1C), and compare this approach to a recently developed Bayesian method ${ }^{40}$.

\subsubsection{Existing motion classification schemes}

Several schemes have been proposed to differentiate between the supposed motion types of single proteins found in (sub)trajectories, such as directed, confined and normal diffusion ${ }^{33,39,41,42}$. Three of these schemes consider classification of pure diffusion states ${ }^{40,43,44}$. Two of these schemes were based on maximum likelihood estimation (MLE). The scheme devised by Ott et al. ${ }^{44}$ employs an MLE approach to classify between diffusive states (also included in our comparison), and uses Hidden Markov Models (HMM) to find the diffusion coefficients of these states. The other scheme relies on a large number of localizations and a prior defined number of diffusion state switching occurrences ${ }^{43}$. With current fluorescence microscopy techniques, it is still impossible to accurately localize many positions to find the current state before the protein switches between states. Furthermore, the amount of diffusion state switching occurrences is not known beforehand, since this switching is a stochastic process. Very recently another scheme has been proposed which uses Bayesian statistics ${ }^{45}$ to discriminate between slow and fast Brownian motion in a spatiotemporal fashion without prior knowledge ${ }^{40}$. This scheme combines information from thousands of short trajectories to identify the number of diffusive states and the state transition rates, and is included in our comparison.

The classification of confined motion, i.e. motion hindered by transient confinement zones, has been discussed elsewhere $e^{3,20,22,38,39}$. We emphasize that our approach is not in contrast to the idea of transient confinement zones. In fact, whether the slow diffusion state originates from pure Brownian motion, a transient confinement, or an immobilization of the protein, cannot be revealed from the limited number of typically acquired positions, and requires other experimental and analysis methods. Although transient confinement or slowed diffusion are closely related, confinement is actually defined as pure diffusive motion restricted by boundaries which cannot be crossed. The confinement area should be of reasonable size such that normal diffusion within this area can still occur. There is no consensus yet on the exact type of motion proteins exhibit.

\subsection{Materials and methods}

We provide an overview of our approach to test classification of segments to dynamic twopopulation diffusion states (Fig. 3.1C), followed by a more detailed description of the individual steps. First, the two diffusion coefficients and their fractional contribution to the trajectories are determined at first using a cumulative distribution function (CDF) fit of the squared displacements ${ }^{20}$. Next we use one of the different local quantification methods, listed in section 
2.5.3-5, which assigns a value to each position in the trajectory. All these methods yield a higher value for a higher diffusion speed. Subsequently, thresholding of these values for the classification is done by taking the $\alpha$-th percentile value of all values found (with $\alpha$ the percentage of step sizes fitted to the first population). For example, when the fraction size of fast diffusion is 0.30 , we set the threshold value such that $30 \%$ of the values are higher than the threshold value. By taking this threshold we perform the classification objectively since the fraction percentage is already accurately determined beforehand from the experimental data itself. To compare the different detection methods in this framework, we tested them using simulated trajectories, where we know the actual diffusion state at each position. The final step in testing the framework is a one-to-one comparison of the found state to the actual (simulated) diffusion state, yielding the classification correctness. We define the classification correctness as the percentage of positions that are correctly classified divided by the total number of classified positions. The state lifetimes $\tau_{1}$ and $\tau_{2}$ found by the analysis are compared with the actual lifetimes for the most promising method.

\subsubsection{Generation of synthetic trajectories}

Two-population diffusion trajectories were generated using MatLAB (The MathWorks, Natick, MA) with the GPUmat toolbox [8]. Each set contained 1,000 trajectories composed of 1,000 frames (positions) in two dimensions with Brownian diffusion steps in between points. The molecule is allowed to change between diffusion states within a trajectory. In more detail, the positions are given by:

$$
\begin{aligned}
& x_{i+1}=x_{i}+R \cdot \sqrt{2 D_{j} \Delta t} \\
& y_{i+1}=y_{i}+R \cdot \sqrt{2 D_{j} \Delta t}
\end{aligned}
$$

where $i$ is the frame number, $R$ is a random number from a standard normal distribution, $D_{j}$ is the diffusion coefficient of the diffusion state $j$, and $\Delta t$ is the time between frames $(\Delta t=40 \mathrm{~ms}$ unless otherwise stated). The dynamical switching behavior between the two diffusion states (e.g. $j$ $=1$, also called fast, and $j=2$, also called slow) is provided by generating subsequent state durations. The duration of the state is determined by taking a random number from an exponential distribution (a Poisson process) with a given characteristic time $\tau_{1}$ and $\tau_{2}$. The diffusion state of all steps in the set is stored, to be able to verify the classification method. Each position $\left(x_{i}, y_{i}\right)$ is given a localization inaccuracy error by adding a random number from a normally distributed pool with standard deviation $\sigma_{\mathrm{xy}}$ in each dimension. The localization error in the x-plane $\sigma_{\mathrm{x}}$ is equal to the error in the y-plane $\sigma_{\mathrm{y}}$, therefore $\sigma_{\mathrm{xy}}=\sigma_{\mathrm{x}}=\sigma_{\mathrm{y}}$.

\subsubsection{Cumulative distribution function of squared displacements}

To find the diffusion constants $D_{1}$ and $D_{2}$ and the fraction $\alpha$ of the first population, we calculate the cumulative distribution function of squared displacements for the complete set of trajectories ${ }^{20}$. Using the complete distribution yields insights into the behavior of the entire population of single molecules, without ensemble averaging effects. As long as there is a large dataset of displacements to build a reliable CDF, it is a straight-forward and reliable method to find the global diffusion coefficients and their fractions. For the two dimensional case, the cumulative distribution function (CDF) for the squared displacements $(\Delta R)^{2}$ for a time lag $\tau=n \cdot \Delta t$ for two diffusion components is given by: 


$$
\begin{gathered}
C D F\left(\Delta R^{2}(\tau)\right)=1-\alpha \cdot \exp \left(-\frac{(\Delta R)^{2}}{4 D_{1} \tau+2\left(\sigma_{x}^{2}+\sigma_{y}^{2}\right)}\right)-(1-\alpha) \\
\quad \cdot \exp \left(-\frac{(\Delta R)^{2}}{4 D_{2} \tau+2\left({\sigma_{x}}^{2}+{\sigma_{y}}^{2}\right)}\right)
\end{gathered}
$$

where $\alpha$ is the fraction corresponding to the motion with diffusion coefficient $D_{1}$. To deal with the localization inaccuracy in the exponent, we determine $D_{1}, D_{2}$ and $\alpha$ for the time lags corresponding to 1 and 2 frames, and fit the exponential terms:

$$
\begin{gathered}
C D F\left(\Delta R^{2}(1)\right)=1-\alpha_{\tau=1} \cdot \exp \left(-\frac{(\Delta R)^{2}}{4 \widehat{D}_{1, \tau=1}}\right)-\left(1-\alpha_{\tau=1}\right) \cdot \exp \left(-\frac{(\Delta R)^{2}}{4 \widehat{D}_{2, \tau=1}}\right) \\
C D F\left(\Delta R^{2}(2)\right)=1-\alpha_{\tau=2} \cdot \exp \left(-\frac{(\Delta R)^{2}}{4 \cdot 2 \widehat{D}_{1, \tau=2}}\right)-\left(1-\alpha_{\tau=2}\right) \cdot \exp \left(-\frac{(\Delta R)^{2}}{4 \cdot 2 \widehat{D}_{2, \tau=2}}\right)
\end{gathered}
$$

which yield the uncorrected diffusion coefficients for each timelag, for example $\widehat{D}_{1, \tau=1}=D_{1, \tau=1}+$ $\sigma_{x y}{ }^{2}$ since $\sigma_{x}{ }^{2}+{\sigma_{y}}^{2}=2{\sigma_{x y}}^{2}$, and similarly $2 \widehat{D}_{1, \tau=2}=2 \cdot D_{1, \tau=2}+\sigma_{x y}{ }^{2}$. Now the estimated diffusion coefficient for the first (and similarly for the second) population corrected for the localization error is:

$$
D_{1}=2 \widehat{D}_{1, \tau=2}-\widehat{D}_{1, \tau=1}=\left(2 D_{1, \tau=2}+{\sigma_{x y}}^{2}\right)-\left(D_{1, \tau=1}+{\sigma_{x y}}^{2}\right)=2 D_{1, \tau=2}-D_{1, \tau=1}
$$

For the fraction $\alpha$ we take the average of the values $\alpha_{\tau=1}$ and $\alpha_{\tau=2}$. In the simulations these two values did not differ by more than a few percent. We have used linear least squares to fit the CDF to the data. Fig. 2.17 shows an example of a CDF fit for motion with two clearly separated diffusion populations.

\subsubsection{Quantification measures}

The next step is to quantify the motion of a molecule for each frame in its trajectory. To this end the trajectories are split in small segments, containing a total number of $N$ subsequent positions (the segment length), and these segments are given a value $W$ by one of the tested quantification measures. Many methods could serve as a measure for slow or fast diffusion. This measure can be, but is not limited to, an estimated diffusion coefficient or confinement index. We have tested the following methods: windowed $\mathrm{MSD}^{34}$, relative confinement ${ }^{35,36}$, the gyration radius $^{37}$, and maximum likelihood estimation (MLE). The values taken are listed in Table 3.1. Besides these windowed measures, we also tested a Bayesian statistics approach ${ }^{40}$ using software made available by these authors. We have already described the mean squared displacement (MSD), relative confinement, and gyration method in more detail in Chapter 2 of this thesis. For this chapter we also made use of a maximum likelihood approach, which was applied as follows.

\section{Maximum likelihood approach}

We have applied a likelihood estimation approach by comparing a window of measured squared displacements, a set of a few single steps $\left\{(\Delta R)_{i}\right\}$, to the expectation value thereof given the distribution function of squared displacements originating from motion with a diffusion constant $D$. For one step of length $\Delta R$, we use $P\left((\Delta R)^{2} \mid D\right)$ to express the chance to find a certain squared displacement given Brownian motion with diffusion coefficient $D$. Since the expectation value of 
one squared displacement is independent of its predecessors, the chances for a tested $D$ can be multiplied for each squared displacement $(\Delta R)_{i}{ }^{2}$, hence the likelihood is given by:

$$
L\left(\left\{(\Delta R)_{i}\right\} \mid D\right)=\prod_{i=1}^{N} \frac{1}{\sqrt{4 \pi\left(D \tau+\sigma_{x y}^{2}\right)}} \cdot \exp \left(-\frac{(\Delta R)_{i}{ }^{2}}{4\left(D \tau+{\sigma_{x y}}^{2}\right)}\right)
$$

where $\tau$ is the time lag, which is 1 frame, and $N$ is the total number of steps in the window. The values for $D$ are taken from the earlier CDF fit. In practice, the localization inaccuracy $\sigma_{x y}$ must be determined by other means first. Here we assumed that this value can be precisely obtained, and we used the true value as used in the generation of the trajectories. Here we determine the likelihood of both states, $L_{1}\left(\left\{(\Delta R)_{i}\right\} \mid D_{1}\right)$ and $L_{2}\left(\left\{(\Delta R)_{i}\right\} \mid D_{2}\right)$, and if $L_{1}>L_{2}$, the segment is classified as state 1 . We could write this as a measure $W$ by:

$$
W(\text { track }, \text { frame })=\frac{L\left(\left\{(\Delta R)_{i}\right\} \mid D_{1}\right)}{L\left(\left\{(\Delta R)_{i}\right\} \mid D_{2}\right)}
$$

We have not taken exposure blur into account ${ }^{46}$. Note that the MLE can also be used to estimate the value of the diffusion constant itself, by maximizing the expectation value by varying the tested $D$ value; the maximum gives the most likely $D$ value ${ }^{43}$.

Table 3.1: Motion quantification measures

\begin{tabular}{|l|c|}
\hline Method & Quantification measure $\boldsymbol{W}$ \\
\hline Windowed MSD & fit of a MSD curve of a segment using first two points in curve \\
\hline Confinement & $1 / L\left(t_{0}+\frac{1}{2} \delta t\right)$ \\
\hline Gyration & $R_{g}$ \\
\hline MLE & $\frac{L\left(\left\{(\Delta R)_{i}\right\} \mid D_{1}\right)}{L\left(\left\{(\Delta R)_{i}\right\} \mid D_{2}\right)}$ \\
\hline
\end{tabular}

\subsubsection{State classification}

When the motion within a segment is quantified, it can be classified as state 1 (corresponding to fast diffusion with coefficient $D_{1}$ ) or as state 2 (corresponding to slow diffusion with coefficient $D_{2}$ ). We allocate the classification of the segment to the center position of that segment, so that a state duration can still be shorter than the segment length. For the maximum likelihood estimation, the classification is performed intrinsically. For the relative confinement and gyration radius methods, the classification is provided by comparing the value $W$ to a threshold value $T$. A segment is classified as state 1 if $W$ is larger than a threshold value $T$, and as state 2 otherwise. The threshold value $T$ is determined by taking all found values $W$, and calculating the $\alpha$-th percentile of these values (with $\alpha$ the percentage of step sizes fitted to the first population). Hence the already known fraction of the diffusion population is used to define the threshold value for the measure to perform the classification.

In the case of the windowed MSD, we slightly altered the way to determine the threshold $T$, due to reasons described in the results section. We used a likelihood approach to calculate the chance that a single value $W$ (calculated for a segment) originates from diffusion with $D_{1}$ or originates 
from diffusion with $D_{2}$. In more detail, a probability density function (PDF) of $W$ is composed for each diffusion constant given the values of the diffusion coefficients $D_{1}$ and $D_{2}$. Examples of such PDFs are shown in Fig. S11A. The threshold value $T$ is chosen as that value of $\mathrm{W}$ where the PDF of $W$ from $D_{1}$ intersects the PDF of $W$ from $D_{2}$ (such that $L_{1}(T)=L_{2}(T)$ ). In this way the segment is classified to the most likely state. The PDF of $W$ for the windowed MSD method for a given diffusion coefficient is calculated as follows. Using a one-population Brownian simulation a trajectory (containing $10^{6}$ positions) is calculated. From this we calculated the values $W$ for all segments in the trajectory. Next the PDF of the found values $W$ is composed. This procedure is performed for both $D_{1}$ and $D_{2}$. Finally, the intersection of these two PDFs is determined.

\subsubsection{Visualization}

After the state classification has been performed, either in simulations or in experimental data, the information obtained can be used for subsequent analysis such as visualization. All the positions of all the molecules in one video recording are used to reconstruct an image, such that the areas are seen where the molecules have travelled. Each individual position (localization) is represented by a color coded dot. The color of the dot depends on the state found at that position and time: red for the slow state, and green for the fast state. This results in diffusion state images at high resolution showing the areas of slow and fast diffusion. We removed immobile trajectories because these were normally found on the glass substrate and not in cells. The filter for immobile trajectories was based on the gyration method applied on a complete trajectory, with the threshold for the reached area defined by a gyration radius of $40 \mathrm{~nm}$, as this corresponded to the apparent area travelled by an immobile molecule due to the localization accuracy. This means that only molecules are displayed that at least once exhibit motion.

\subsubsection{Cell culture}

All cell culture materials were obtained from PAA Laboratories (Pasching, Austria) unless stated otherwise. MCF7 cells, a human breast cancer cell line, and plasmid coding for SNAP-EGFR were a gift from Jenny Ibach (Max Planck Institute in Dortmund, Germany). Cells were cultured in Dulbecco's Modified Eagle's medium supplemented with 10\% FBS and penicillin/streptomycin at $37^{\circ} \mathrm{C}$ with $5 \% \mathrm{CO}_{2}$. Before measurements, the cells were transferred to CellView dishes product \#627870 (Greiner Bio-one, Alphen aan den Rijn, The Netherlands), grown overnight, transfected with SNAP-EGFR using Effectene (Qiagen, Venlo, The Netherlands), and then starved overnight the day after transfection in medium without FBS. Labeling of the SNAP-EGFR proteins was done by incubating the cells for 1 minute with 400nM of SNAP-Surface 549 (New England BioLabs, Ipswich, MA, USA) in 0.5\% BSA. Measurements were performed in PBS buffer with added magnesium and calcium (PAA Laboratories, product H15-001).

\subsubsection{Microscopy}

Measurements were performed on a microscope with an Olympus PlanApo 100x/1,45 Oil TIRF objective using TIRF illumination. For excitation a 532nm laser $(400 \mathrm{~mW})$ from Pegasus Shanghai Optical Systems (Pegasus Lasersysteme, Wallenhorst, Germany ) was used. All the light filters were obtained from SemRock (Rochester, NY). The infrared light produced by the laser was not sufficiently suppressed, therefore the green laser light passed an FF01-543/22 filter. The excitation and emission is split by an FF494/540/650-Di01 dichroic mirror. The emission light is filtered with an NF03-532/1064E notch filter and an FF01-580/60 bandpass filter. Fluorescence images were acquired using an Andor iXon EM+ DU-897 back illuminated EMCCD with an acquisition time of $9 \mathrm{~ms}$ and a kinetic cycle time of $38 \mathrm{~ms}$ ( $25.8 \mathrm{fps}$ ). The microscope stage was heated with a sample heating plate and the objective was heated with a ring heater to $35-37^{\circ} \mathrm{C}$. 


\subsubsection{Tracking}

To obtain the trajectories from the raw videos, we used tracking software developed by others ${ }^{6,47}$. The settings used for the cost matrices in this software can be found in the appendix of Chapter 2 .

\subsection{Results}

\subsubsection{Performance of different quantification measures}

First we validated our approach by simulating the extreme case of well-separated diffusion constants with $D_{1}=40 \cdot D_{2}$ and long state durations. We obtained a correctness of over $95 \%$ for windowed MSD, and over 99\% for the other methods, as expected for clearly distinct motion. Next we tested the different quantification measure methods for diffusion classification, and studied the influence of different segment lengths therein. Therefore we simulated (at $25 \mathrm{fps}$ ) four cases with two diffusion states with different state lifetimes and localization accuracy. The cases were chosen to provide a challenging and realistic situation for discrimination of the two diffusion constants from experimentally obtained single molecule trajectories. The diffusion constants were chosen to reflect relatively slow membrane receptors (unpublished observations): $D_{1}=0.06 \mu \mathrm{m}^{2} / \mathrm{s}$, and $D_{2}=0.015 \mu \mathrm{m}^{2} / \mathrm{s}$. For other ratios of diffusion coefficients, see Fig. S4. We chose our switching settings close to the values found for the EGF receptor: $\tau=300-900 \mathrm{~ms}$. The localization accuracy depends on the number of photons recorded from a molecule per frame. The chosen localization accuracies are typical values observed for quantum dot labels $\left(\sigma_{x y}=20 \mathrm{~nm}\right)$ or fluorescent protein labels $\left(\sigma_{x y}=40 \mathrm{~nm}\right)$, whereas organic dyes will often be somewhere in between these values. The accuracy does not only depend on the number of photons acquired for localization, but also on the labeling strategy. For instance, antibodies are large macromolecules and their flexibility leads to a lower localization accuracy.

Fig. 3.2 shows the performance of diffusion state classification for different quantification measures of local diffusion together with the influence of the segment length chosen. For the windowed MSD method, we display the correctness when using the first three points of the MSD curve in the fit, as this gave the best correctness in all the simulation cases. For the first simulation (case A) we chose the localization accuracy $\sigma_{x y}=40 \mathrm{~nm}$ and the state lifetimes were both set to 300 ms. To study the influence of the localization accuracy $\sigma_{x y}$ alone, in simulation case B this parameter was lowered to $20 \mathrm{~nm}$. In simulation cases $C$ and D, only the switching behavior was altered compared to case A to be able to test the influence of the state lifetimes. When the two lifetimes are not equal (case C), this clearly changes the diffusion fractions, such that there are an unequal number of molecules in each state on average. The two diffusion coefficients and their fractions were not assumed to be known beforehand, analogous to experimental data. These state parameters are found for each simulation by a fit to the cumulative distribution function (CDF) of squared displacements. The results show that an optimal choice of the segment length is needed to yield the best classification correctness. The optimal segment length depends to a large extent on the state lifetimes and also on the particular quantification measure used in the state classification.

We find that the non-diffusion based gyration evolution method is the most accurate measure for diffusion state classification in the simulation cases with localization accuracy of $40 \mathrm{~nm}$ (cases A, C and D). In these cases, for equally sized diffusion populations (case A and D), the gyration based classification scores better than classification using any other method. When the diffusion populations are not equally sized (case $\mathrm{C}$ ), the gyration based classification scores almost as well as the computationally much more expensive Bayesian method. In simulation case B with a localization accuracy of $20 \mathrm{~nm}$, the Bayesian method and MLE based classification score better than the other methods. This trend continues when there is no localization inaccuracy; simulations for this case showed the MLE method then scores $81 \%$ correct compared to $74 \%$ for 
the gyration based classification. In practice however, the localization will rarely be better than 20 $\mathrm{nm}$, due to the limited number of photons and labeling biochemistry related issues. In the case of slow state switching behavior (case D), the gyration based classification is at these state-switching rates already close to its best possible performance with these diffusion coefficients and localization inaccuracy; increasing the average state duration to infinity only resulted in $3 \%$ improvement in correctness. The classification correctness for the windowed MSD achieved when another number of points in the MSD curve is taken to perform the fit can be found in Fig. S2. The classification correctness when we set a minimum state duration for a number of frames can be found in Fig. S3.

After classification of the diffusion inside trajectories, the lifetime of the states can be determined by composing a histogram of state durations and fitting the lifetime. We determined the distribution of the slow and the fast state lifetime found by the gyration based classification in trajectories of simulation case A (Fig. S5). We found that the state lifetimes fitted are shorter than the actual (simulated) lifetimes, especially for longer state lifetimes. More simulations showed that a trend of changing the state lifetimes in the simulation is reflected in the lifetimes found, although larger lifetimes (as in case D) are significantly underestimated, especially when the correctness is below $85 \%$.

Although the correctness percentages provide a measure of the classification performance, the exact number might not give a feeling for how useful such a classification is. We will return to this point in the section on identifying the zones of slow diffusion. Clearly the percentage must be higher than $50 \%$ to have any relevance, since this percentage would also be obtained by a completely random state allocation.

FIGURE 3.2 Correctness of the twopopulation classification by different quantification measures for different simulated cases (A-D in the corresponding panels). The correctness of the different quantification measures is plotted against the segment lengths used in the classification. The Bayesian method does not use segments, and its result is shown with a dashed line. In all simulation cases $D_{1}=0.06$ $\mu^{2} / \mathrm{s}$ and $D_{2}=0.015 \mu \mathrm{m}^{2} / \mathrm{s}$. $(A)$ Simulation case $A$ has a localization inaccuracy $\sigma_{x y}$ of $40 \mathrm{~nm}$ and short state lifetimes $\left(T_{1}\right.$ and $\left.T_{2}\right)$. (B) This simulation case differs from case $A$ only by a lowered localization inaccuracy $\sigma_{x y^{*}}(C)$ This simulation case differs from case $A$ only by having a longer fast state lifetime $T_{1}$. (D) This simulation case also has a longer slow state lifetime $T_{2}$.
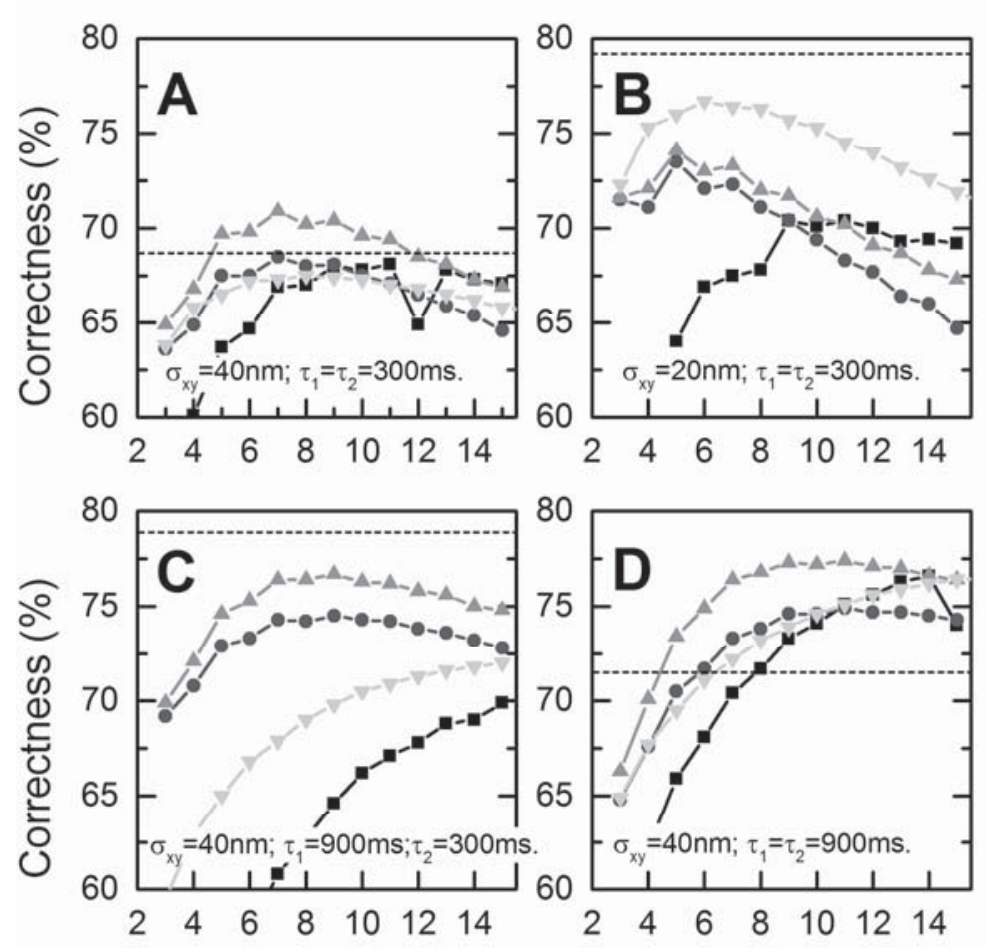

Segment length

Segment length

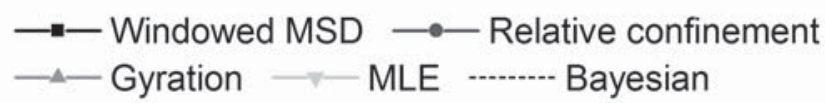




\subsubsection{Optimized threshold}

The windowed MSD, relative confinement, and gyration measures use a threshold for classification. We noticed that for the windowed MSD, the classification correctness was not around its maximum with the threshold at the $\alpha$-th percentile, especially for unequally sized diffusion populations (Fig. 3.3A). In the case of simulation case $\mathrm{C}$ (i.e. with unequally sized diffusion populations), the best result when using the windowed MSD as the quantification measure would be to classify every position as fast diffusion, and thereby scoring around $75 \%$ correct (i.e. the fast fraction size), however such classifications would not provide any information. The other methods score the same correctness at the 100th percentile threshold by definition, but these methods score a higher correctness with a threshold at the $\alpha$-th percentile. For the windowed MSD method, we therefore used another threshold. We instead compared the likelihoods that a value $W$ for a segment originates from diffusion with diffusion coefficient $D_{1}$ or from diffusion with diffusion coefficient $D_{2}$. In other words, the threshold was set at the intersection of the probability density functions of the values obtained with the windowed MSD for diffusion from each diffusion state separately (detailed in the methods section). We verified that the windowed MSD using likelihoods for state classification indeed performed with a higher correctness compared to when windowed MSD values are compared to a threshold using the $\alpha$-th percentile. Likelihood methods do not regard the fraction size $\alpha$ to determine the most likely state for a segment, therefore the MLE and the Bayesian methods are not included in Fig. 3.3A.

A

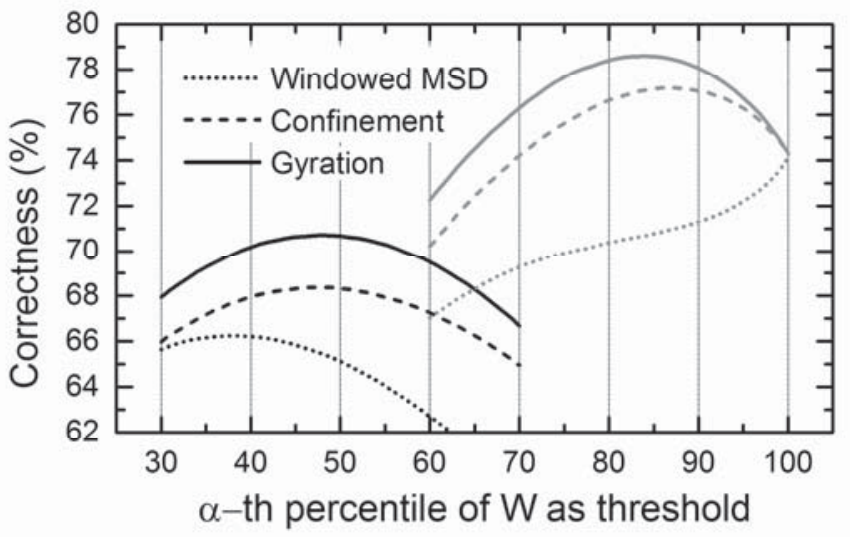

B

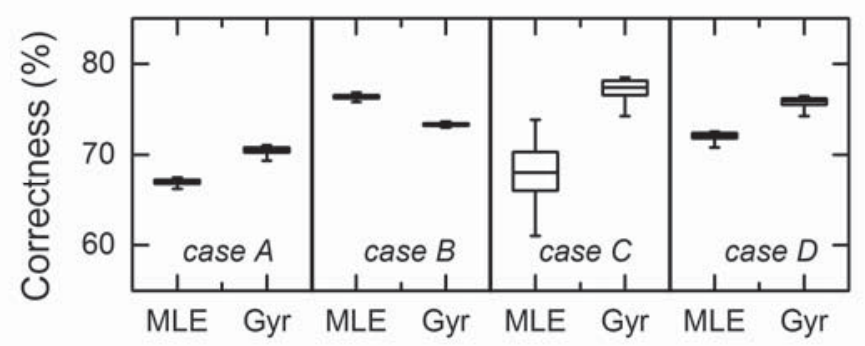

FIGURE 3.3 (A) The correctness

dependence on the choice of the state classification threshold for simulation case $A$ (left curves) and case $\mathrm{C}$ (right curves). The threshold varied with the $\alpha$-th percentile value of calculated quantification measure values $W$. In these simulation cases the fraction size for fast diffusion $\alpha=0.5$ and $\alpha=$ 0.75 (case $A$ and $C$ respectively), and the classification was performed with a segment length of 7 frames. The windowed MSD method does not yield optimal correctness with a threshold using the $\alpha$-th percentile, yet the optimal threshold does not provide any information either. Therefore the threshold selection for this method was altered to using the likelihood that the found value for a segment corresponds to either of the two diffusion states. $(B)$ Robustness of the MLE and gyration based classification. When simulations with 100 trajectories and 1,000 frames are repeated 100 times, variations can come from the accuracy of the CDF fit. The boxplot show the resulting distribution $(5,25,50,75,95 \%)$ of the obtained classification correctness using MLE and gyration (Gyr) method for all simulation cases (A-D, left to right) due to this effect. 


\subsubsection{Classification robustness}

The values for the classification performance in Fig. 3.2 and Fig. 3.3A are average values that are obtained for many classifications. Since one simulation entailed 1,000 trajectories of 1,000 frames, the statistical noise averaged out between simulations with the same diffusion parameters. However, analyzing the results of one simulation is not sufficient to predict the robustness of the method, since the robustness in the correctness also depends on other aspects of the classification framework. For example, the correctness depends in large extent on the fitted diffusion constants and fractions obtained from the CDF fit. Therefore we tested whether small perturbations in the CDF fit influenced the obtained correctness for the MLE and gyration method. We used a segment length of 7 frames to classify 100 simulations to obtain the distribution of the correctness. In each simulation we used the simulation settings of case A, except for the number of trajectories in the simulations, which was lowered to 100 trajectories (a realistic number of molecules in a tracking experiment). In this way, fewer displacements are available for the CDF fit, and therefore the fitted values have a larger spread in subsequent simulations. The resulting correctness distributions (Fig. 3.3B) shows that neither of the two methods is influenced dramatically by slightly perturbed CDF fits, except for the case where both fractions are not equally distributed (case C). The spread in that case is especially large in the MLE method.

\subsubsection{Identifying the zones of slow diffusion}

After state classification of the diffusion, super-resolution like images of the diffusion states can be reconstructed. When the distribution of slow diffusion zones is not randomly distributed, the proposed approach for diffusion state classification should be able to detect these zones. To validate this application, we performed simulations of 200 trajectories where the diffusion state was spatially defined. The regions with slow diffusion were defined 1 pixel wide (corresponding to $120 \mathrm{~nm}$ ), and were separated by 5 pixels. The separations were the regions of fast diffusion. The
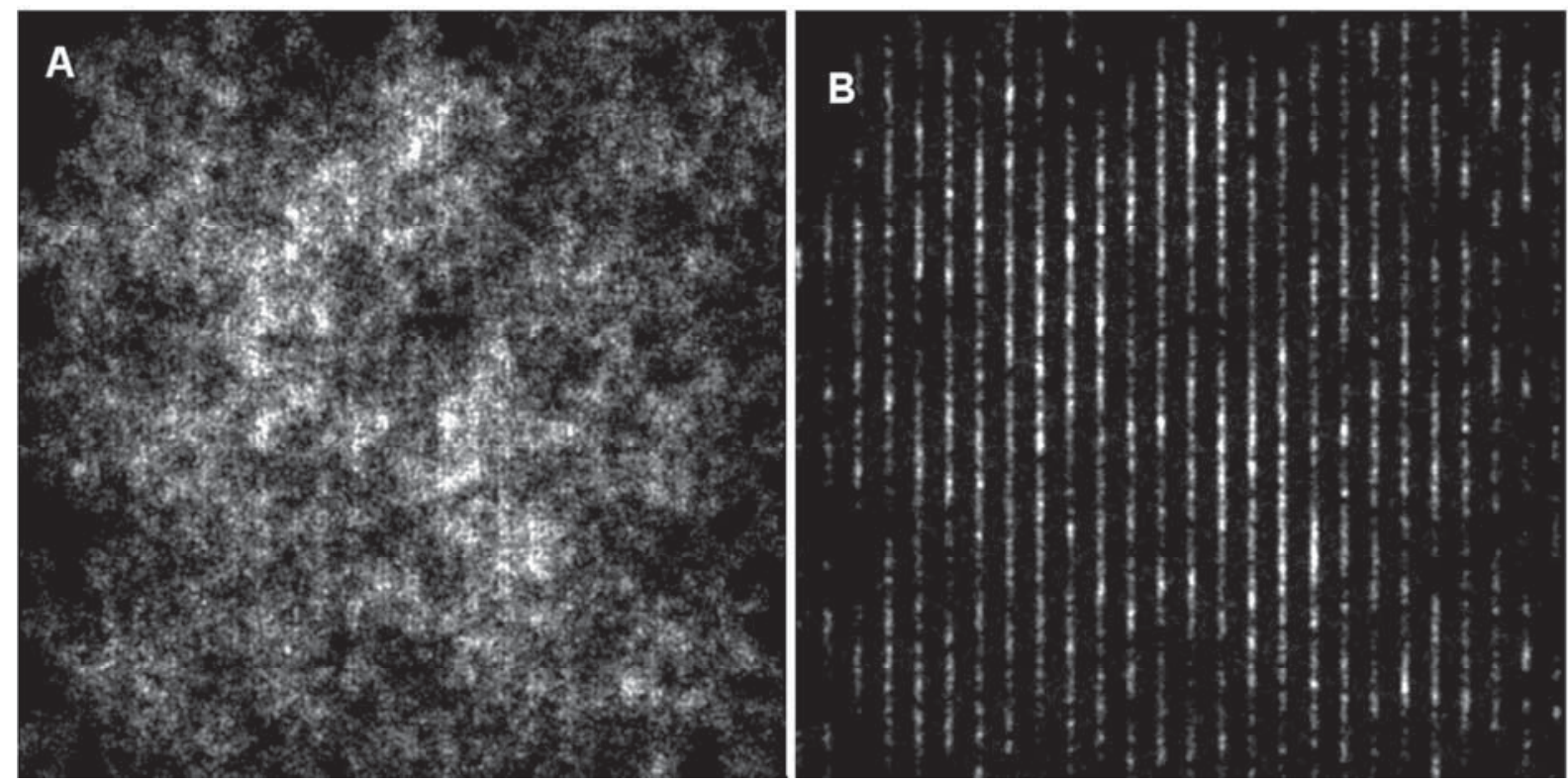

FIGURE 3.4 Diffusion states classified by the gyration method visualized at high resolution images displaying the regions in the fast state $(A)$ and slow state $(B)$. The simulation was designed with spatially defined zones (vertical lines) for diffusion states. Here the slow state regions were $120 \mathrm{~nm}$ wide and are separated by $600 \mathrm{~nm}$ wide fast state regions. The regions of the slow diffusion state are suitably classified and clearly visible. The dimension of the image is $15 \times 15 \mu \mathrm{m}$, and the image is reconstructed at a resolution of 30 $\mathrm{nm} / \mathrm{pixel}$. 
diffusion value in the fast region was chosen $D_{1}=0.10 \mu \mathrm{m}^{2} / \mathrm{s}$, and in the slow regions it was chosen $D_{2}=0.01 \mu \mathrm{m}^{2} / \mathrm{s}$, with the localization accuracy $\sigma_{x y}=20 \mathrm{~nm}$. These settings were chosen to represent a typical membrane protein imaged utilizing bright fluorophores. The resulting state lifetimes were around $300 \mathrm{~ms}$. We performed the diffusion state classification using the gyration quantification measure with a segment length of 4 frames (Fig. 3.4 and Video 1). Simulations with the diffusion state parameters mentioned showed that this is the optimal segment length. The correctness of gyration based classification was $85 \%$. The figure shows that the performance is more than adequate to visualize the spatial diffusion state organization described. The actual states of the simulation and the same reconstruction image using MLE based classification are shown in Fig. S7. For comparison, when the motion and the state switching is completely random, such as in simulation case A, the reconstruction map also shows apparent zones (Fig. S6). However, these zones are only caused by the randomness of Brownian movement.

\subsubsection{Example of classification applied to EGF receptor}

The advantage of spatiotemporal resolved state classification is the possibility to observe where the molecules have travelled in which diffusion state. Reconstructed videos may also reveal whether multiple diffusion populations are originating from a pool of molecules exhibiting either diffusion state, or a pool of molecules transiently making transitions between the states. The lifetimes of the states can be determined as well from the histogram of state durations.

To demonstrate the potential of the application of diffusion state classification, we performed a gyration based classification on experimental single molecule tracking data. We recorded fluorescence images of fluorescently labeled EGF receptor in MCF7 cells by utilizing SNAP-tag (Video 2). In this video recording we detected on average 210 fluorescent molecules per frame (Fig. S8D). The localization accuracy in our video is close to $40 \mathrm{~nm}$. The diffusion constants $D_{1}$ and $D_{2}$ and the fraction size $\alpha$ of the fast state from all displacements are determined using a fit to the CDF of squared displacements (Fig. S8). We obtained $D_{1}=0.112 \pm 0.001 \mu \mathrm{m}^{2} / \mathrm{s}$, and $D_{2}=$ $0.008 \pm 0.001 \mu \mathrm{m}^{2} / \mathrm{s}$ with $\alpha=0.69 \pm 0.03$ (the given errors are $5-95 \%$ confidence intervals of the fit). Intercellular differences are larger than the errors of fitting the CDF. We used a segment length of 7 frames, which performs best according to simulations for the given diffusion parameters (classification correctness of 86\%). State lifetimes (or kinetic rates, the inverse lifetime) were obtained by combining the state durations from five recordings of EGF receptor (unliganded) to get enough statistics (Fig. S9).

After diffusion state classification, we reconstructed the diffusion state video (Video 3) and diffusion state images at high resolution (Fig. 3.5 and Fig. S10). In these images we clearly see distinct zones of slowed diffusion. Furthermore we see cellular structures such as filopodia at the boundaries of the cell, and possibly collapsed filopodial structures on the lower membrane. The presence of EGF receptor in filopodia was expected, since EGF receptor undergoes retrograde transport in filopodia ${ }^{48,49}$.

\subsection{Discussion}

\subsubsection{The optimal segment length}

The optimal segment length correlated to the state lifetime in our simulations. For example, the optimal 7 frames in simulation case A and B corresponds to the average state lifetime of $300 \mathrm{~ms}$ (7.5 frames), and when the state lifetimes increase (simulation case $\mathrm{C}$ and $\mathrm{D}$ ), the optimal segment length also increases. The optimal segment length also varies for the different quantification measures, especially when the state lifetimes increase (simulation case $C$ and D). Since the state lifetimes are not known before the classification, it would be preferable when the results do not 


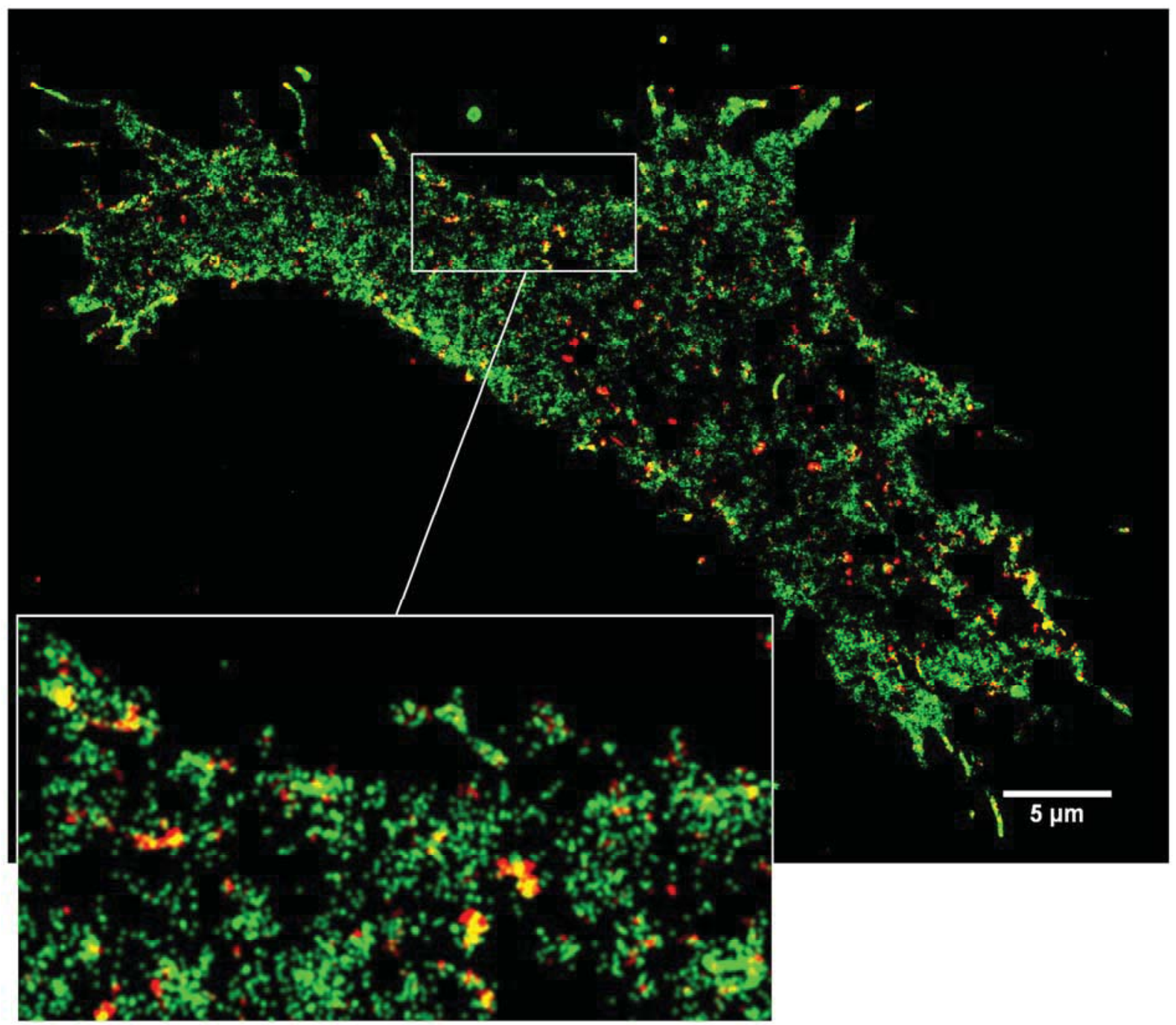

FIGURE 3.5 The spatial distribution of the diffusion states exhibited by EGF receptors in an MCF7 cell. The reconstructed image shows the areas where receptors were classified in the fast diffusion state as green, and areas where receptors were classified in the slow diffusion state as red. For a region at the periphery of the cell a zoomed image (inset) shows clear regions of slow diffusion. The image also shows that the receptor is associated with certain cellular structures such as filopodia. The classification is performed using the gyration method with a segment length of 7 frames. The resolution of the reconstructed image is $30 \mathrm{~nm} / \mathrm{pixel}$.

vary much for different segment lengths. We can see that the gyration method with a segment length of 7 frames scores near optimal in almost all the cases simulated. Therefore, a good strategy would be to perform the classification first with the gyration method and a segment length of 5-7 frames. This might provide a good first classification with an adequate correctness. Later, the classification may be repeated with a different segment length more suitable to the state lifetimes found to obtain an optimized classification or a verification of the reconstructed diffusion state images.

\subsubsection{Diffusion state classification}

We noticed that the gyration method has higher correctness and is more robust compared to the other tested methods, especially in situations with higher localization errors $\left(\sigma_{x y}=40 \mathrm{~nm}\right)$. The reason may be found in the fact that more information is used to determine the gyration radius compared to the value calculated with the windowed MSD or the MLE method. In the calculation of the gyration, the information of the distances between all the segments positions is taken into 
account unlike in MLE. Therefore the gyration method not only considers the distance between points, but also the relative locations of the positions. For example, for pure diffusion it is unlikely that a particle would move in only one direction; the MLE would however not detect this as it only considers subsequent distances, whereas the gyration method will. The spatial information of relative location of the positions is only considered in the gyration and confinement methods, which might be the reason why these methods scored higher in classification of the two diffusion states. In the windowed MSD method, the optimum result is obtained when the fit to find the diffusion value is obtained from information only up to a time lag of 3 frames. This means that in the case of the windowed MSD calculation the distance information from the first to the last point is not considered, whereas this is taken into account in the gyration method. However, when the positions are more precisely known (i.e. low localization error), the MLE and Bayesian based methods start to outperform the gyration method. An explanation for this might lie in the fact that these methods make use of averaged distances of single steps. The average value of single steps in a segment is apparently precise enough to find the most likely underlying diffusion coefficient. Without localization errors, the (windowed) MSD also scores best when only single steps (corresponding to a time lag of 1 frame) are considered. Our approach with the windowed MSD is then in fact equivalent to the MLE method ${ }^{43}$. Another reason why the windowed MSD scores lower than other methods might be because MSD values can be negative, especially with higher localization errors. It is not clear what the chance is that negative values represent a slow or a fast state (we classified these values as slow), such that negative values cannot be adequately classified.

Interestingly, it remains an open question whether the gyration based classification method is the optimal quantification measure for the cases simulated, or that another quantification measure can be invented that outperforms gyration based classification. We saw that classification based on the maximum likelihood of the average of single step sizes is outperformed by gyration based classification, hence MLE does not yield the optimal performance to this problem. How to best combine all the positional information of a segment remains an open question.

Although we demonstrated the state classification for a two population system, the framework and the methods can also be used for more than two populations. The CDF fit should then be adjusted for multiple populations, and thresholds can similarly be set at the found $\alpha$-th percentile. This expansion can be benchmarked using similar simulations situations as we have presented here. Other quantification methods can also use this framework for benchmarking the method in a realistic context of single molecule experiment on plasma membrane receptors. In this chapter we have tested prevalent analysis methods for quantification of the local diffusion. Although the confinement and gyration methods have been developed in the context of confinement, they had never been applied for quantification of pure Brownian motion, whereas we showed that these methods outperform classical methods to classify segments to a diffusion state.

Michalet $^{34}$ has discussed the practicality of using an windowed MSD. He argued that the segment length must be chosen small enough so as to measure local behavior and not averaged global behavior, yet large enough to be sufficiently accurate as too small windows have a broad distribution in output values. He therefore concluded that this method can rarely provide reliable estimates of the diffusion coefficients, and can only show a difference in multiple orders of magnitude, even for windows of 100 points. Although this conclusion is valid in the case of exceedingly low diffusion coefficients, such as $D=10^{-4} \mu \mathrm{m}^{2} / \mathrm{s}$ in his example, we showed that a windowed MSD can still be useful in diffusion state classification. However it is indeed not as powerful as the other methods tested.

We chose to use the CDF fit approach to find the global diffusion values. Another approach to obtain the diffusion coefficients has been described using Bayesian statistics ${ }^{45}$ and a hidden Markov model. This method finds the different (and hidden number of) diffusion states with their diffusion coefficients ${ }^{40}$. Although this method yields accurate results, it also requires about a 
thousand fold more computational time on our computer, whereas a CDF fit is comparably accurate. Concerning the accuracy of the quantification measure, we neglected the influence of the exposure time on these values. While this leads to underestimation of the value of the diffusion coefficient due to the averaging blur during exposure ${ }^{1,46}$, we only used the values to classify states; the exposure time effect should not have much influence on this classification.

In this chapter we only regarded spatiotemporal analysis methods, as especially the spatial information may yield important information, such as where a molecule is interacting. Therefore we have excluded image correlation spectroscopy methods, such as $\mathrm{PICS}^{50,51}$, which do not require tracking, and a Monte Carlo approach ${ }^{38}$. Also, we only looked at alternating diffusion states, and not at active transport or confinement, as explained before.

\subsubsection{Application in live cells}

The application of the gyration based diffusion state classification approach demonstrated that the correctness achieved is sufficient to identify spatial zones of slowed diffusion within cellular structures. Such zones are perticularly important as they reflect the regions where the protein interacts with other proteins or cellular compounds. The physiological meaning of the slow diffusion zones in our example of EGF receptor remains an open question at the moment. They could be related to cytoskeletal structures or regions where the receptor exhibits internalisation. For other proteins we might find another distribution of membrane patches of slowed diffusion, and specific diffusion states near other cellular structures such as actin or microtubuli might be seen. In that case, single molecule trajectories may indicate a role of such cellular structures in the signaling of a protein studied when analyzed by diffusion state classification.

Diffusion state lifetimes obtained by this classification framework seem less informative, as they tend to be underestimated due to short periods of incorrect state classification (Fig. S5). To reliably obtain changes in state lifetimes, a high classification correctness ( $>85 \%)$ seems essential. State lifetimes can however be used as a test to confirm that the diffusive populations found (using the CDF fit) are lasting longer than the timescale of the sampling rate (time between frames). States with extremely short lifetimes are probably caused due to issues with multiple intermixed diffusive states, and further diffusion classification is unreliable in that case.

\subsection{Conclusion}

In summary, we have introduced a new strategy for spatiotemporal classification of twopopulation diffusion, and compared methods to be used for this classification. We have validated our proposed diffusion state classification approach by testing with simulations and showed possible applications, such as determining diffusion state lifetimes and composing diffusion state images. The key feature of the proposed framework is that a diffusion estimator is the logical choice but not necessarily the best way to discriminate and classify segments to two diffusion states. When we have determined the diffusion coefficients and their fractions present in the motion of all the molecules (e.g. using a CDF fit of the squared displacements $s^{20}$ ), there is no need to find a local diffusion coefficient. What remains is the need to classify the local motion to one of the found diffusion states. The found fraction size $\alpha$ can be used to perform objective thresholding of the local quantification measures. This avoids relying on subjective manual thresholding in segment based methods to detect transitions between motion patterns ${ }^{39}$, such as relative confinement ${ }^{4,36}$.

We have found that the gyration method is best used for diffusion state classification when the localization error (due to photon shot noise and the finite proximity of the fluorescent dye to the protein) is on the order of $40 \mathrm{~nm}$, whereas MLE or Bayesian methods are preferred in the case of localization errors of $20 \mathrm{~nm}$ or less. Although the differences in the resulting classification correctness are small, the robustness of the gyration method is higher than the MLE method, 
especially when a limited number of displacements are available for the CDF fit. Furthermore, the Bayesian method was about a thousand fold slower on our computer, while it outperforms the gyration based classification only marginally. For realistic plasma membrane receptor motion, the optimal setting of the gyration method requires a segment window of 4 to 7 frames; the method then classifies $70 \%$ to $90 \%$ correct, depending on the exact characteristics of the motion. Simulations with spatially organized diffusion states demonstrated that this is adequate to observe spatial organization of diffusion states. The estimated correctness for experimental data may be determined by performing simulations as demonstrated. When the diffusion states are visualized at high resolution at their position in live cells, such diffusion state images may aid in identifying spatially separated zones of the occurring states on the membrane of the cell. Zones of slowed diffusion are an indication of interactions with the protein studied. We showed that such zones exist for EGF receptors within cellular structures. The image also showed static or slowly dynamic cellular structures, such as filopodia.

In conclusion, new biophysical insight could be acquired from spatiotemporal information of protein mobility. Such information can be obtained through the proposed diffusion state classification approach. We expect that the visualization of zones of altered diffusion of proteins on top of other cellular structures will help in providing a better understanding in the organization of the plasma membrane and the role of the cytoskeleton in protein signaling. Spatial diffusion classification will be a valuable tool for obtaining more insight in the complex protein interactions in live cells.

\section{Acknowledgment}

The authors would like to thank Kasper van Zon for discussions about the state classification likelihood, and Jenny Ibach for providing the SNAP-ErbB1 plasmid. Furthermore we thank Peter Relich and Dr. Keith Lidke for sharing their single molecule tracking software with us.

\section{Grants}

This work is supported by an EU NanoSci E+ grant via STW grant 11022-NanoActuate. 


\title{
Supporting Material
}

\section{Classification of dynamical diffusion states in single molecule tracking microscopy}

\author{
Peter J. Bosch ${ }^{1}$ \\ Johannes S. Kanger ${ }^{2}$ \\ Vinod Subramaniam ${ }^{1,2,3}$
}

1. Nanobiophysics, MESA+ Institute for Nanotechnology and MIRA Institute for Biomedical Technology and Technical Medicine, University of Twente, PO Box 217, 7500AE Enschede, The Netherlands

2. MIRA Institute for Biomedical Technology and Technical Medicine, University of Twente, The Netherlands

3. Present address: FOM Institute AMOLF, Science Park 104, 1098 XG Amsterdam, The Netherlands 


\section{Supporting Figures}

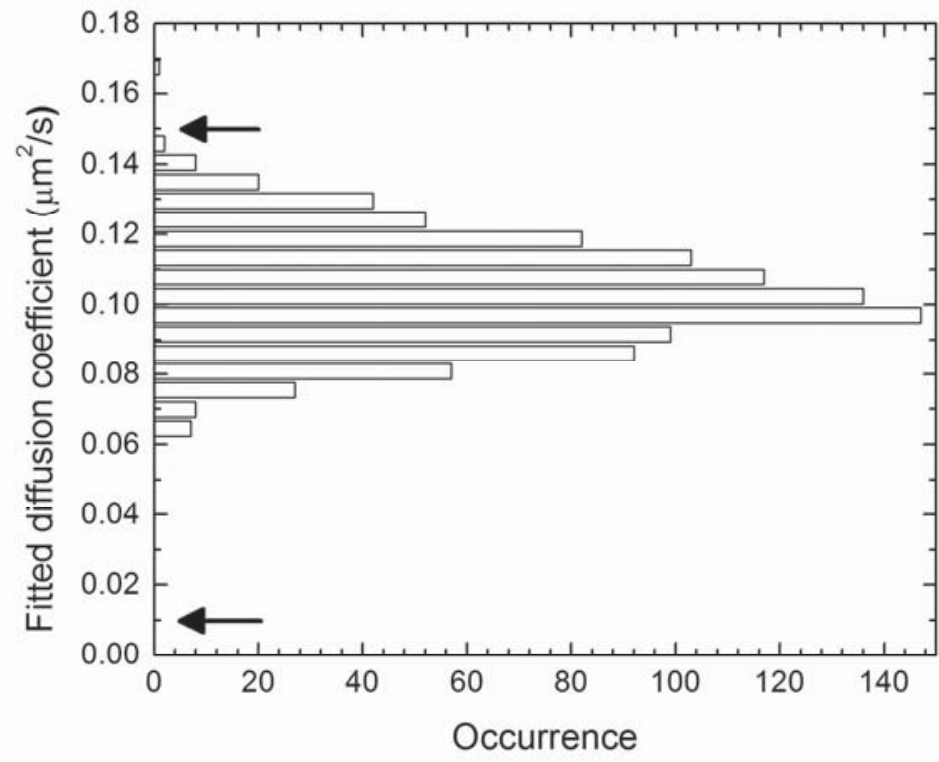

FIGURE S1 A conventional full-trajectory MSD analysis of a two-population diffusion systems leads to an apparent distribution of diffusion values, easily resulting in erroneous conclusions for this motion system. The true motion system is a two-population diffusion system with diffusion coefficients of 0.15 and $0.01 \mu \mathrm{m}^{2} / \mathrm{s}$, as indicated by the arrows in the graph. For this graph, 1000 trajectories consisting of 500 frames (hence relatively long trajectories) were simulated. The trajectories are analyzed using the conventional full-trajectory MSD method, where the first 4 points of the MSD curve were used following the "rule of thumb" rule to fit the diffusion constant. The found diffusion constants and the apparent spread both incorrectly describe this twopopulation situation. The MSD analysis should therefore only be used for homogeneous (one-population) motion. The discussion further in the supplementary material discusses the accuracy of determining the diffusion constant for a one-population system using the full-trajectory MSD method. 
A

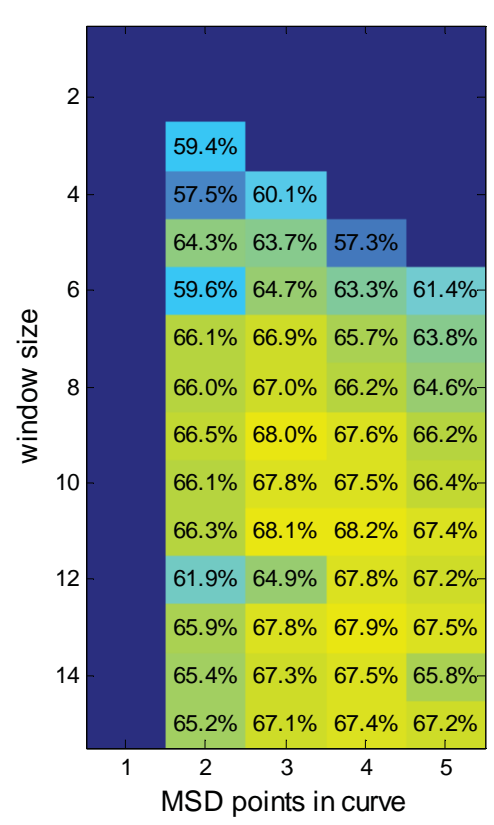

C

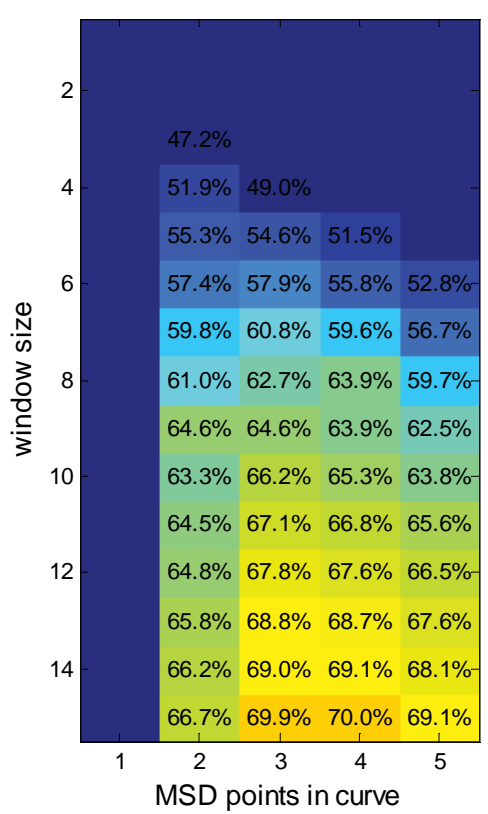

B

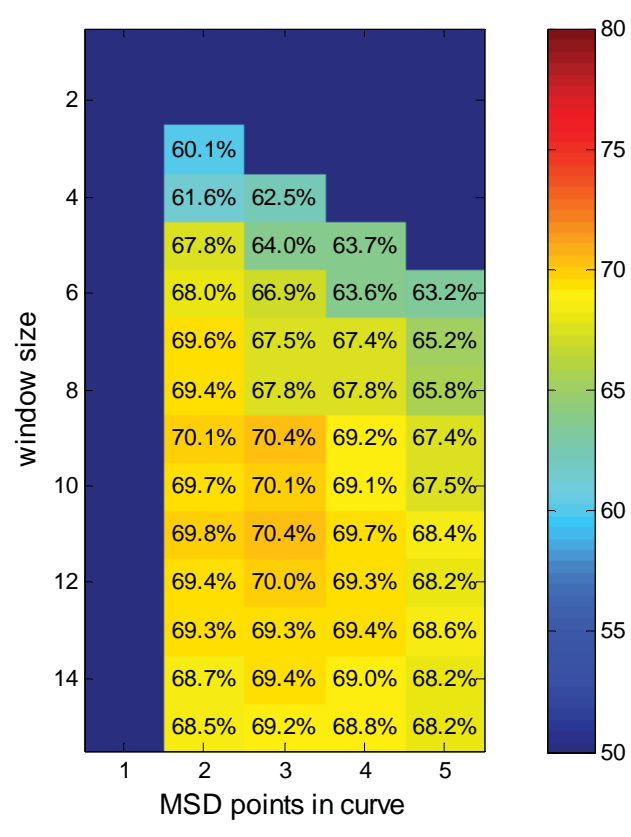

D

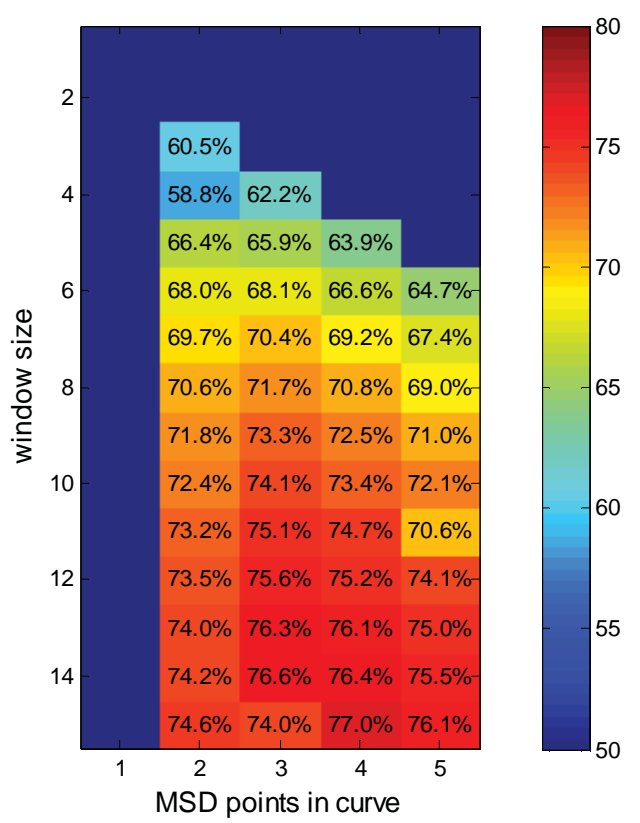

FIGURE S2 Correctness dependence of window length and of the number of points in the MSD curve used to fit the diffusion value. (A) Simulation case A has a localization inaccuracy $\sigma_{x y}$ of $40 \mathrm{~nm}$ and short state lifetimes $\left(T_{1}=T_{2}=300 \mathrm{~ms}\right)$. $(B)$ This simulation case differs from case A only by a lowered localization inaccuracy $\sigma_{x y}=20 \mathrm{~nm}$. (C) This simulation case differs from case A only by having longer fast state lifetimes $\left(\tau_{1}=900 \mathrm{~ms} ; T_{2}=300 \mathrm{~ms} ; \sigma_{x y}=40 \mathrm{~nm}\right)$. (D) This simulation case also has longer slow state lifetimes $\left(I_{1}=900 \mathrm{~ms} ; \tau_{2}=900 \mathrm{~ms} ; \sigma_{x y}=40 \mathrm{~nm}\right)$. The color bar aids in reading the classification correctness percentage. 
A

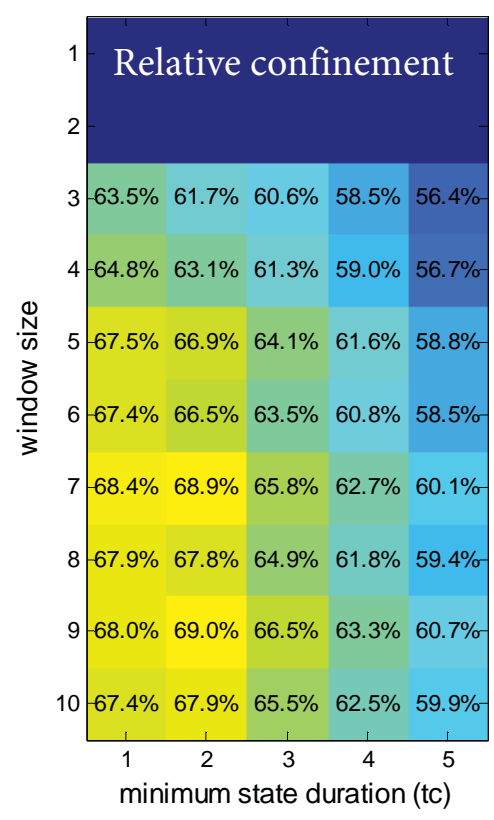

B

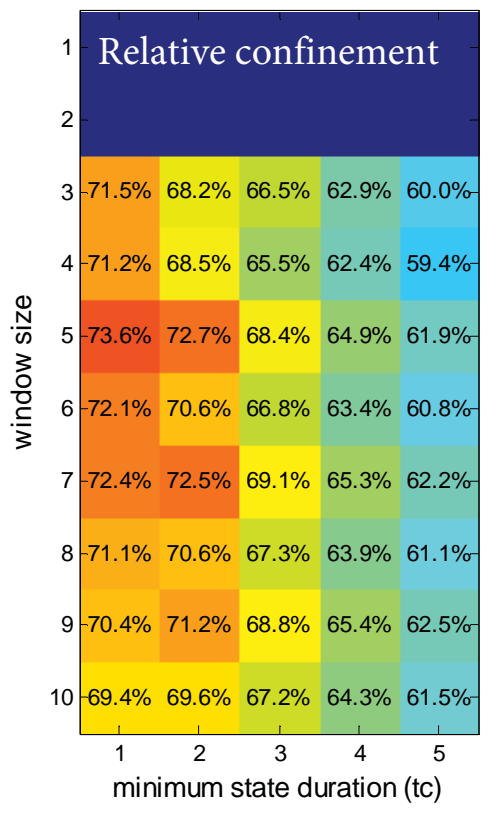

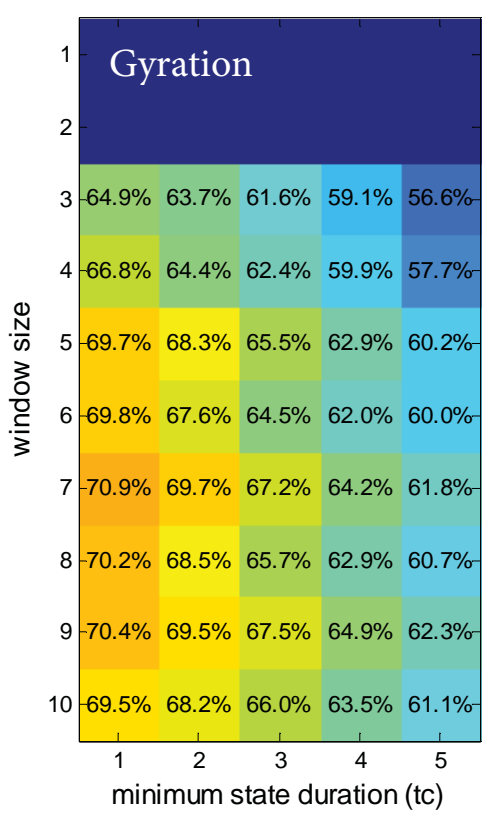
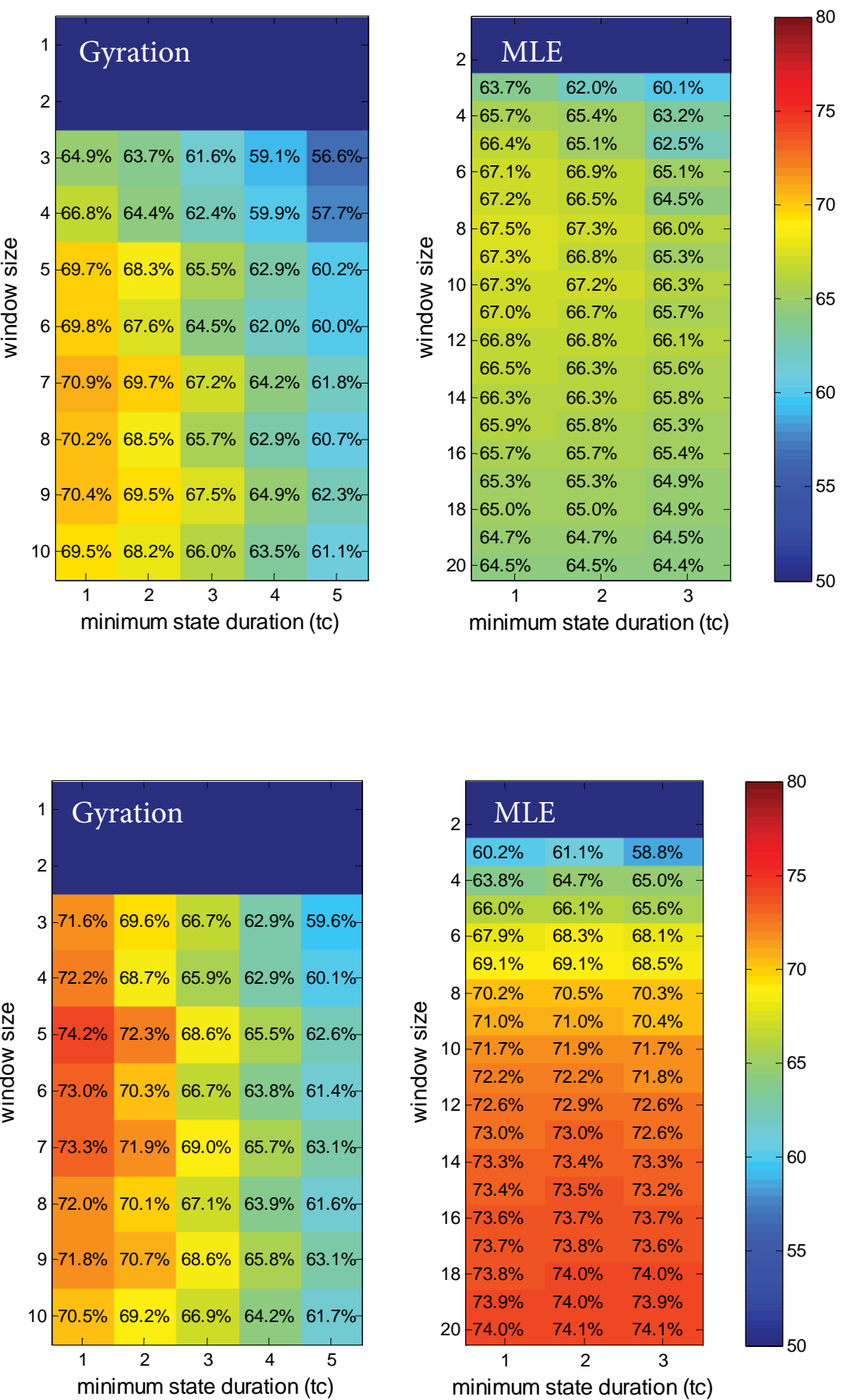


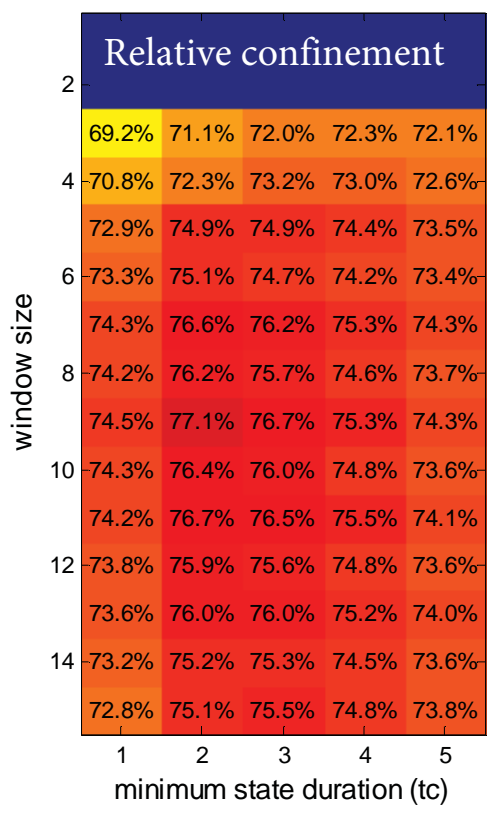

D

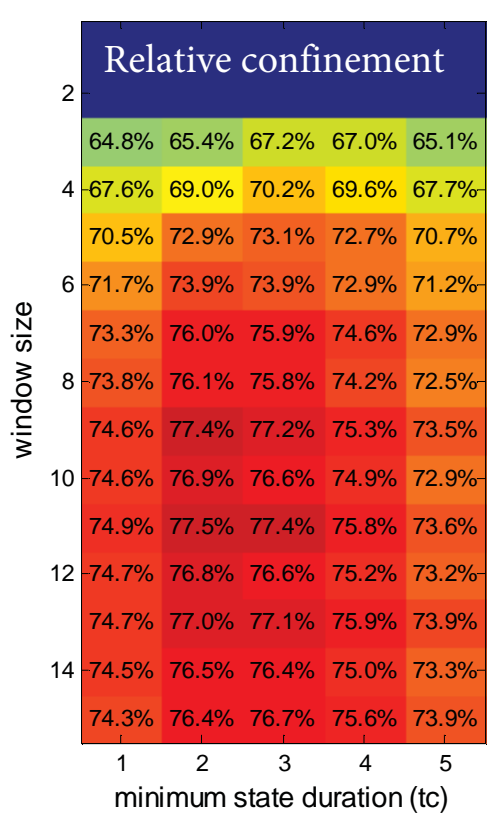

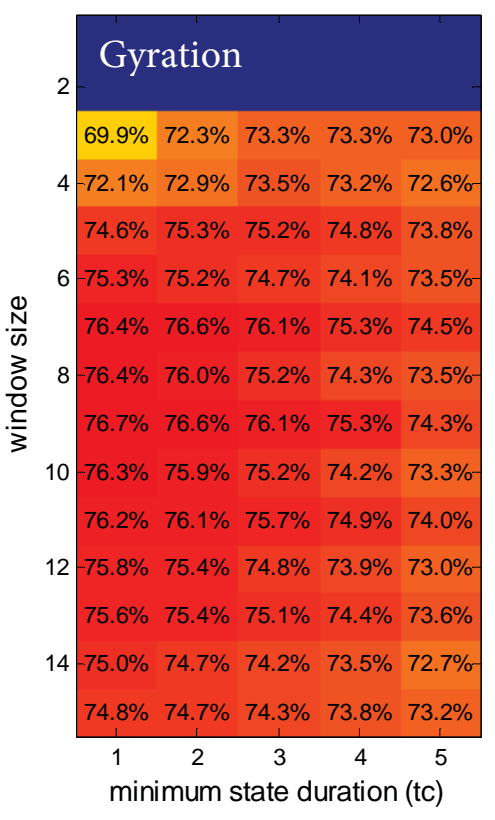
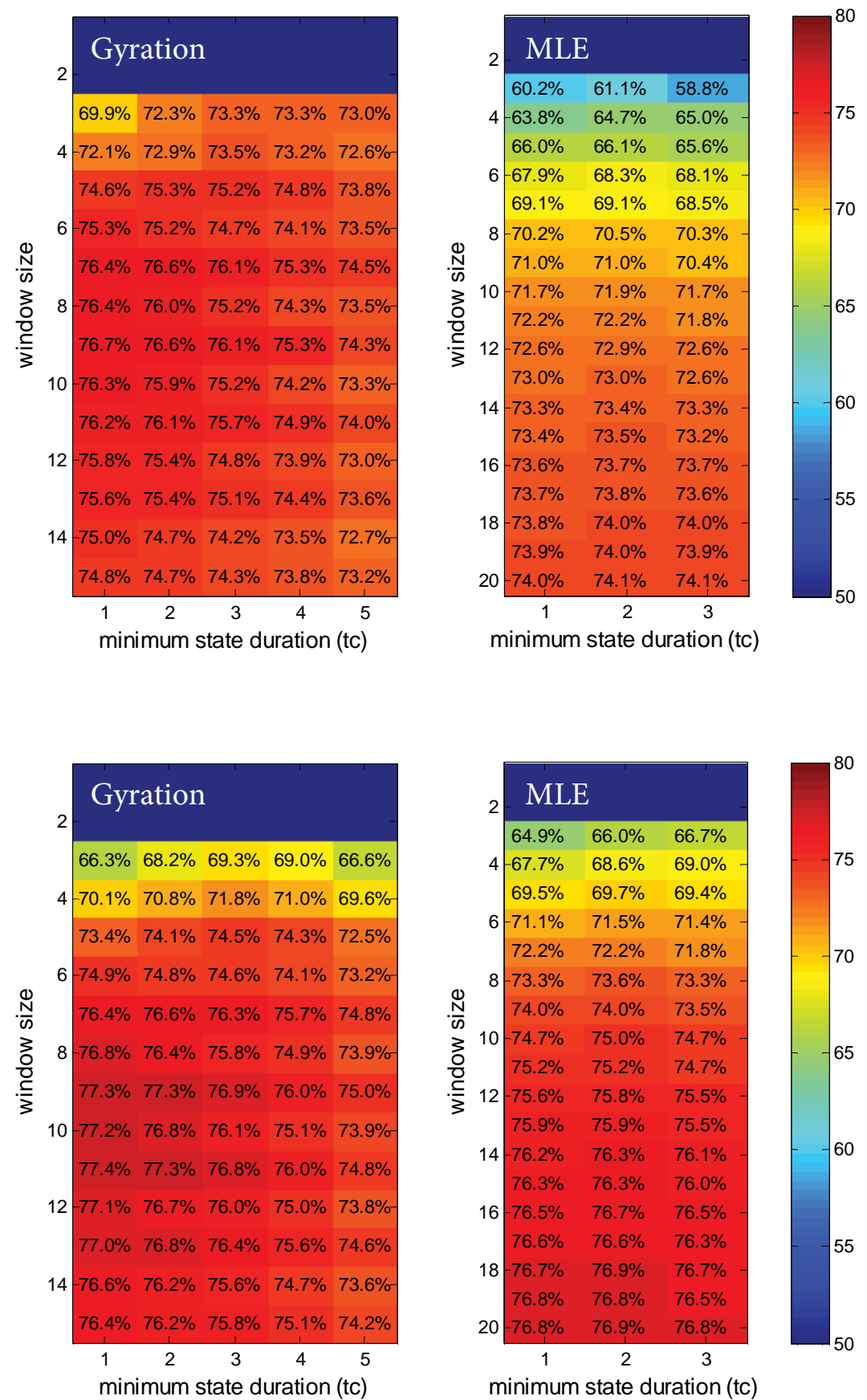

FIGURE S3 Effect of a minimum state duration filter on classification correctness. Since all the above methods make use of segments, the state classification does not allocate exact time points of a trajectory to a state, but to a window of several time points. The state of this window is classified at the center time point of the window, and thereby each time point has its own state allocation based on different but correlated data sets. Now the state classification is defined at all timepoints except for the beginning and end of a (sub)trajectory. Since the window is several frames long, state durations shorter than the segment length (window size) may appear illogical, and therefore we also tested whether filtering out short state durations leads to better correctness for different classification methods. In most simulation cases (as described in the main text) this was not the case, and we have therefore looked only at unfiltered state classifications in the main text. $(A-D)$ Classification correctness for simulation case A-D. The color bar aids in reading the classification correctness percentage. 


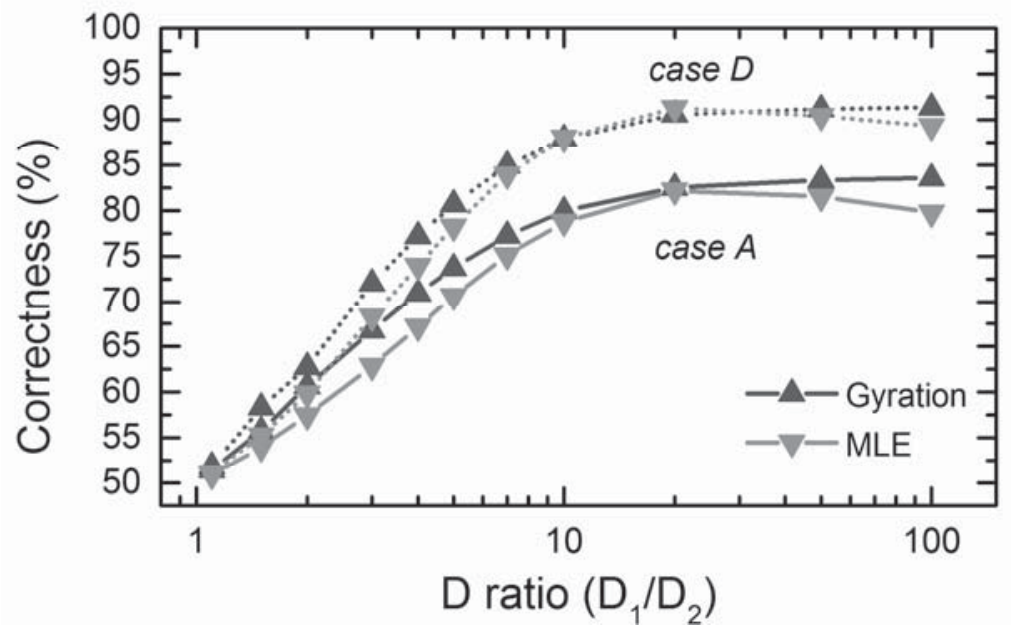

FIGURE S4 Correctness of the two-population classification for different ratios for $D_{1}$ and $D_{2}$. $D_{2}$ was kept fixed at $0.015 \mu \mathrm{m}^{2} / \mathrm{s}$. Shown are the results for a gyration and MLE based classification for simulations of cases $A$ and $D$ (with changing $D_{1}$ ). We find that ratio of 4 in our simulation cases was indeed a challenging situation, and that with ratios larger than 10 the best achievable classification are obtained. The classification also depends on other factors as shown by the different results for the two cases shown.
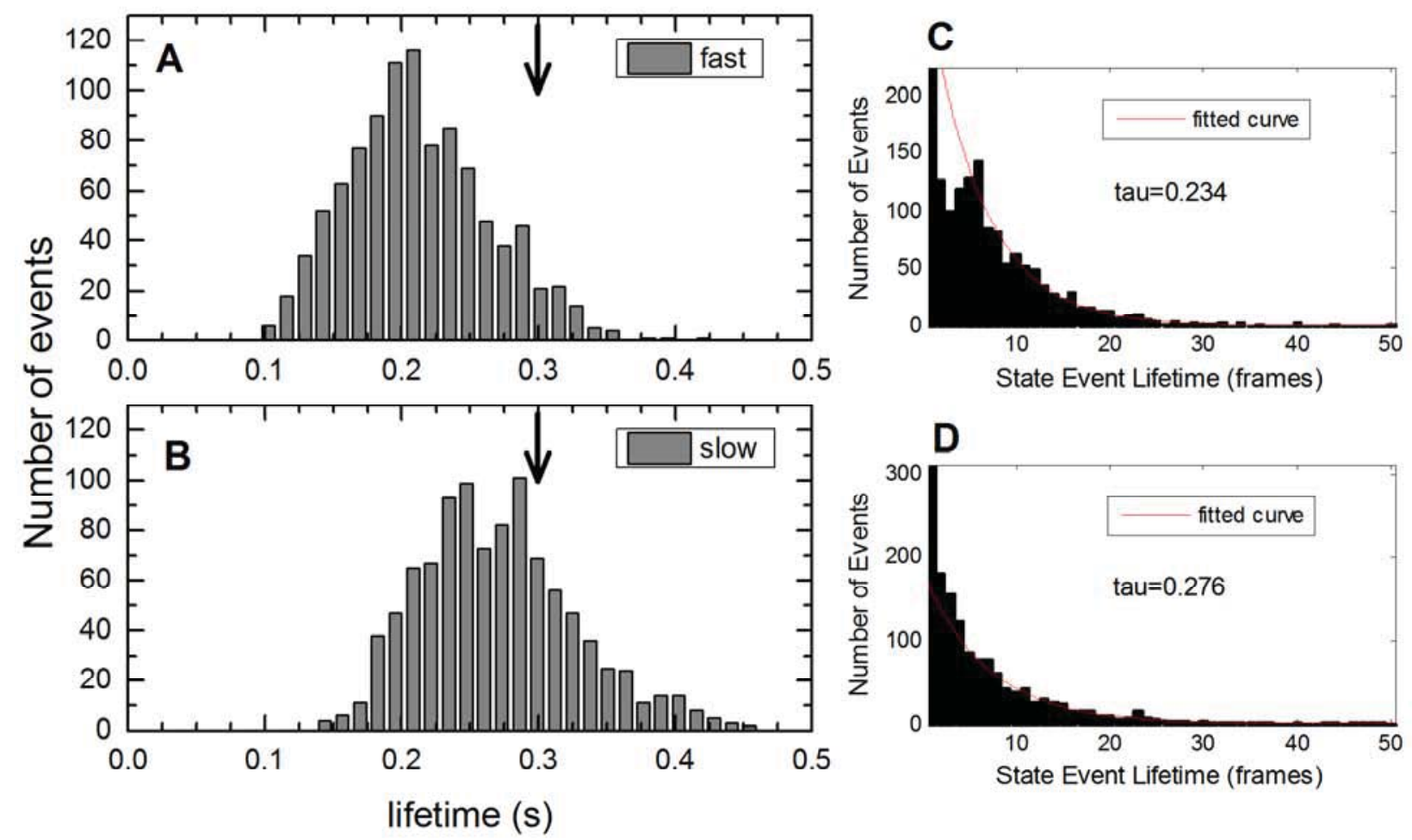

FIGURE S5 The distribution of lifetimes found by the gyration based classification method simulation case A. The simulation consisted of 20 trajectories and was repeated 1,000 times to yield the distribution of lifetimes of the slow state $(A)$ and the fast state $(B)$. The gyration method used a segment length of 7 frames. The arrows indicate the true lifetime of both states. $(C)$ Example of a lifetime fit of the fast state from one simulation. This fit is only performed over state events larger than 5 frames. (D) Example of a fit of the slow state. This fit is only performed over state events larger than 5 frames. Although the lifetimes found are not too far off in this case, in cases with longer state durations (such as case D), the lifetimes found are significantly underestimated. 


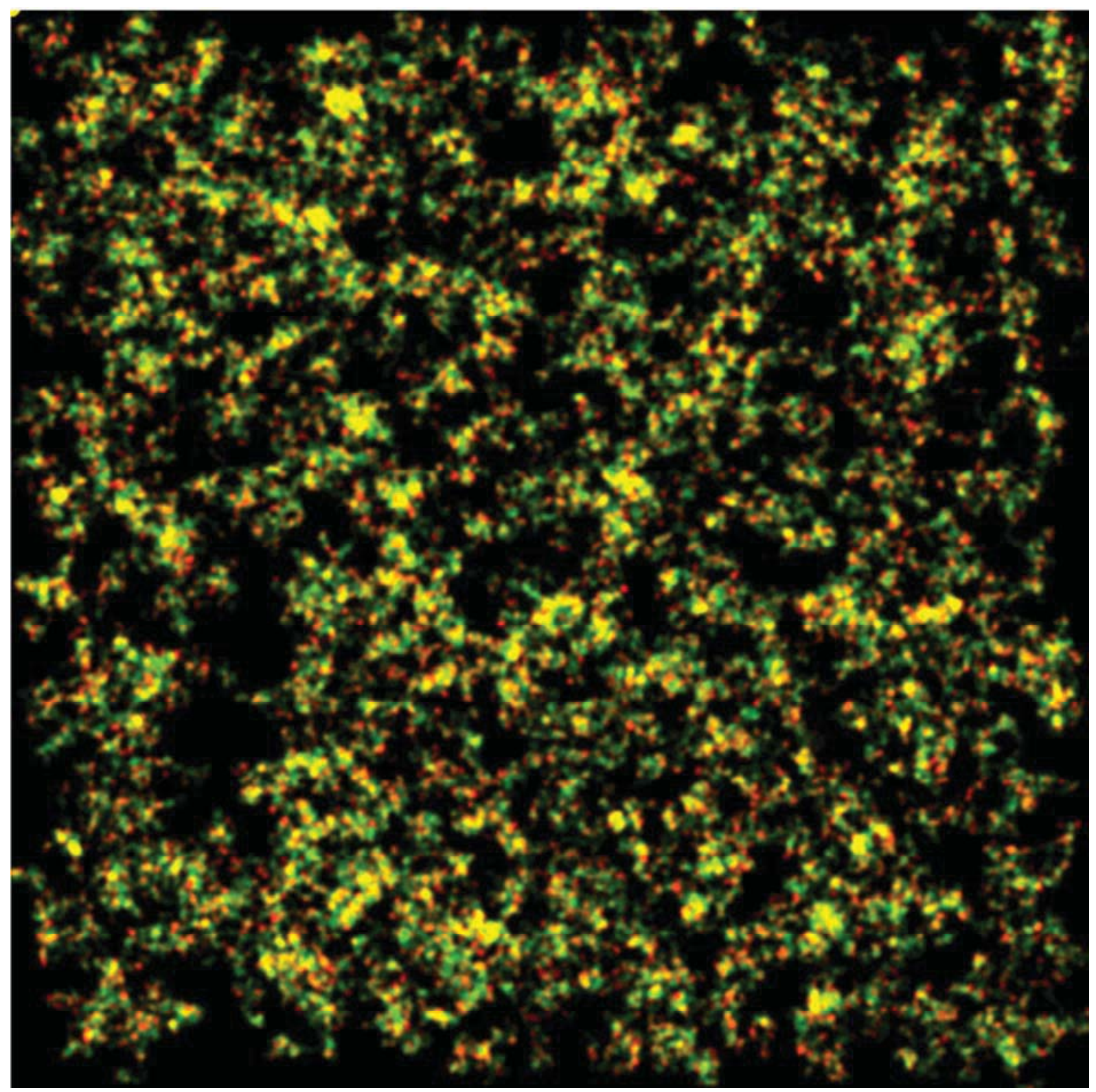

FIGURE S6 Image reconstruction of the state classification distribution of simulation case A. In this case there were as many slow as fast states. The dimension of the image is $55 \times 55 \mu \mathrm{m}$, and the image is reconstructed at $60 \mathrm{~nm} / \mathrm{pixel}$. 


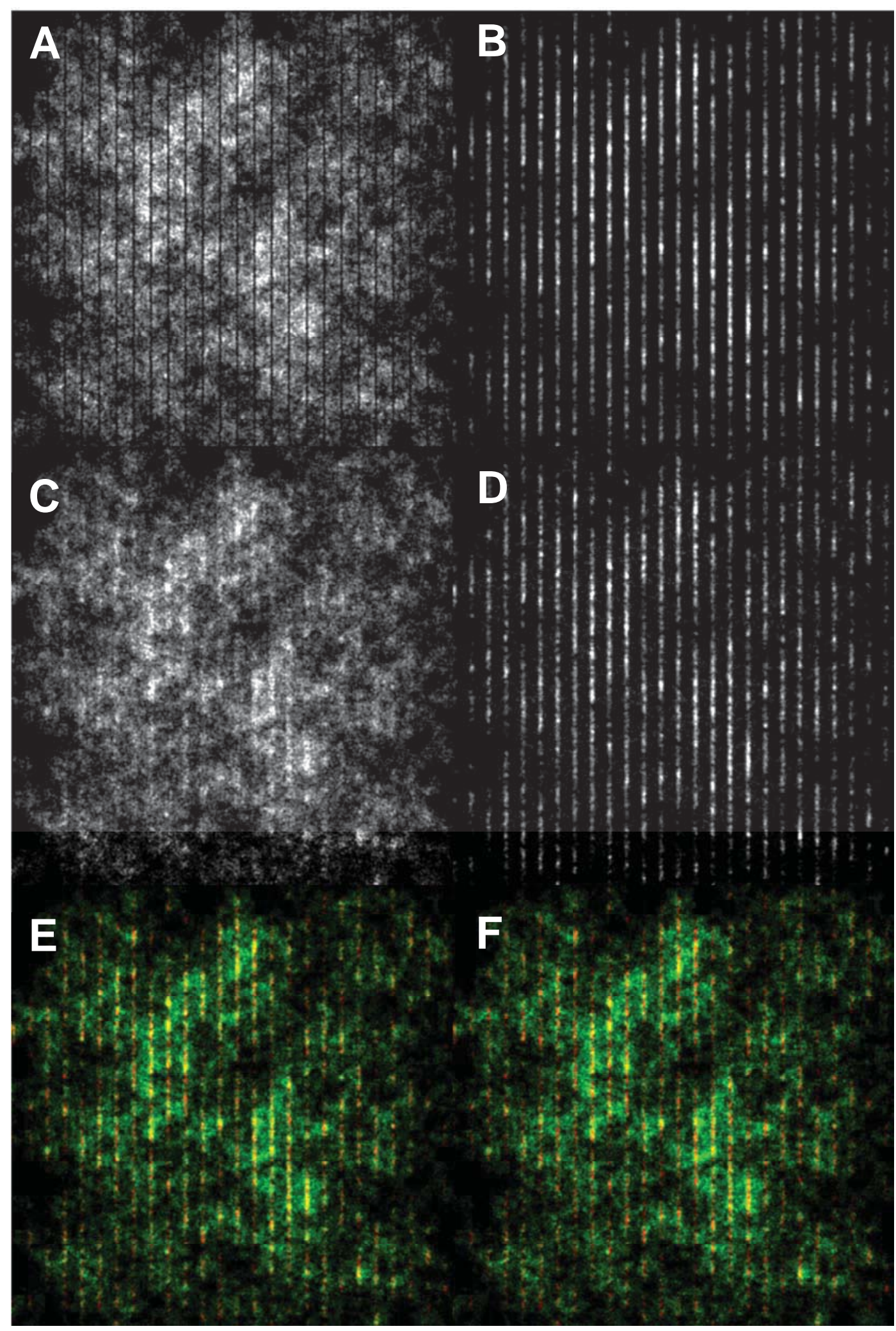

FIGURE S7 Image reconstructions of the state classification distribution of a simulation with spatially defined diffusion states. (A) Simulation (actual) image of fast state. $(B)$ Simulation (actual) image of slow state. (C) Fast state image found by MLE method. $(D)$ Slow state image found by MLE method. (E) Colour image of states map as found by the MLE method. Green represents fast state, and red represents slow state. $(F)$ Colour image of states map as found by the gyration method. Green represents fast state, and red represents slow state. A segment length of 4 frames was used in both classification methods (this value yielded the highest correctness). The dimension of the image is $15 \times 15 \mu \mathrm{m}$, and the image is reconstructed at $30 \mathrm{~nm} / \mathrm{pixel}$. 

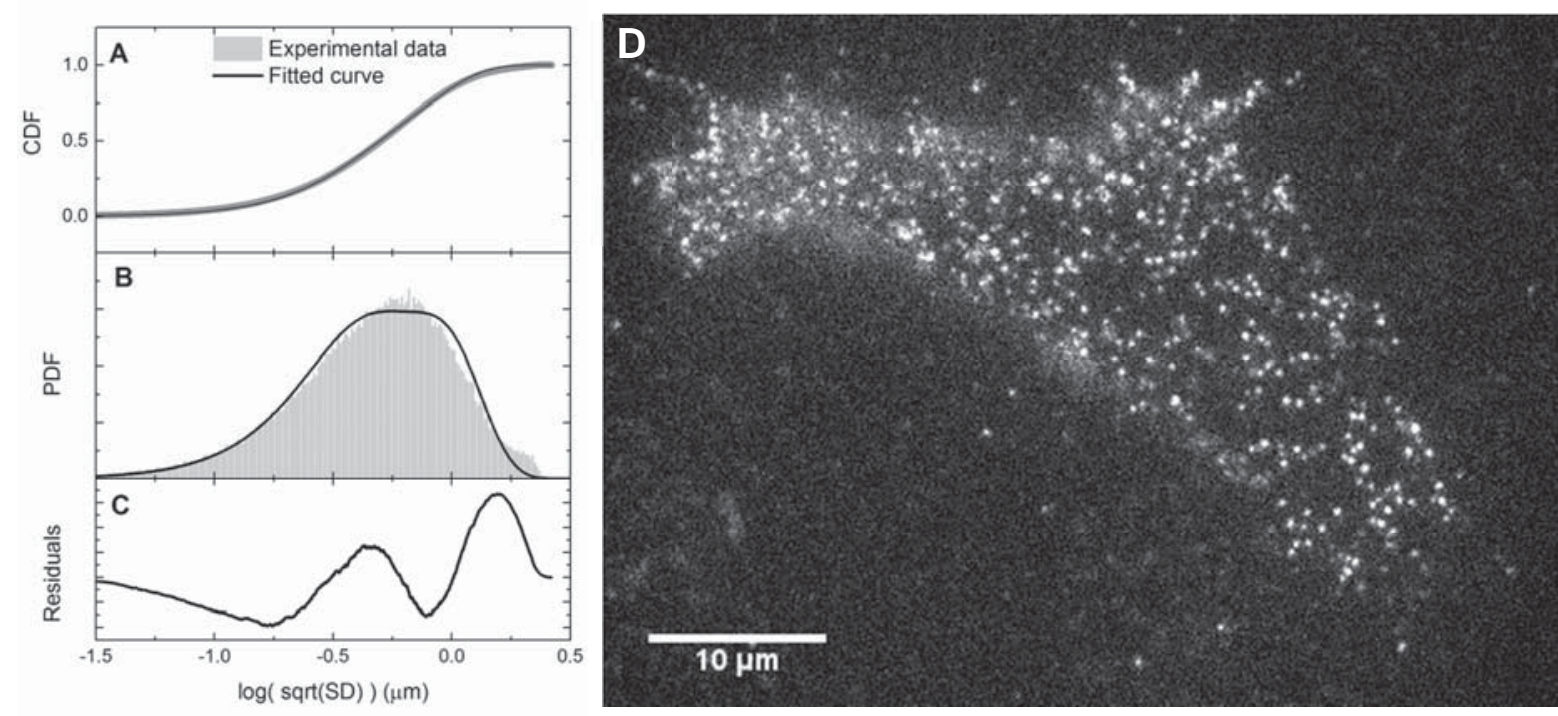

FIGURE S8 The proposed approach for diffusion state classification applied to experimental data. $(A)$ The CDF fit, with corresponding PDF $(B)$ and residuals $(C)$, for the motion of EGF receptor in an MCF7 cell shows that the model of two-population Brownian diffusion is a suitable motion model. (D) An example of a single molecule fluorescence frame recording of EGF receptor in an MCF7 cell. The image has not been modified or filtered.

FIGURE S9 Histograms of unliganded EGF receptor state lifetimes for the fast state (left) and the slow state (right) in frames (video was recorded at $25 \mathrm{fps}$ ), determined by gyration analysis. The characteristic lifetime is determined from an exponential fit of state durations longer than 5 frames, since the use of a segment length of 7 frames does not correctly resolve shorter lifetimes. The state duration will be underestimated because of random incorrect state classification.

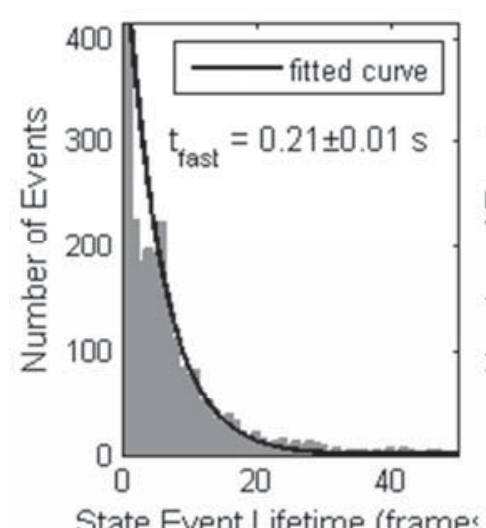

State Event Lifetime (frames

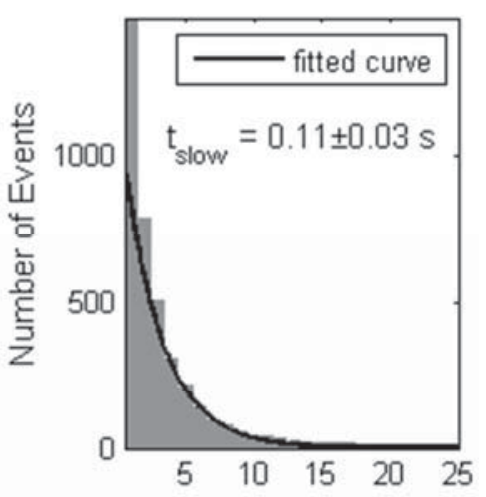

State Event Lifetime (frame: 

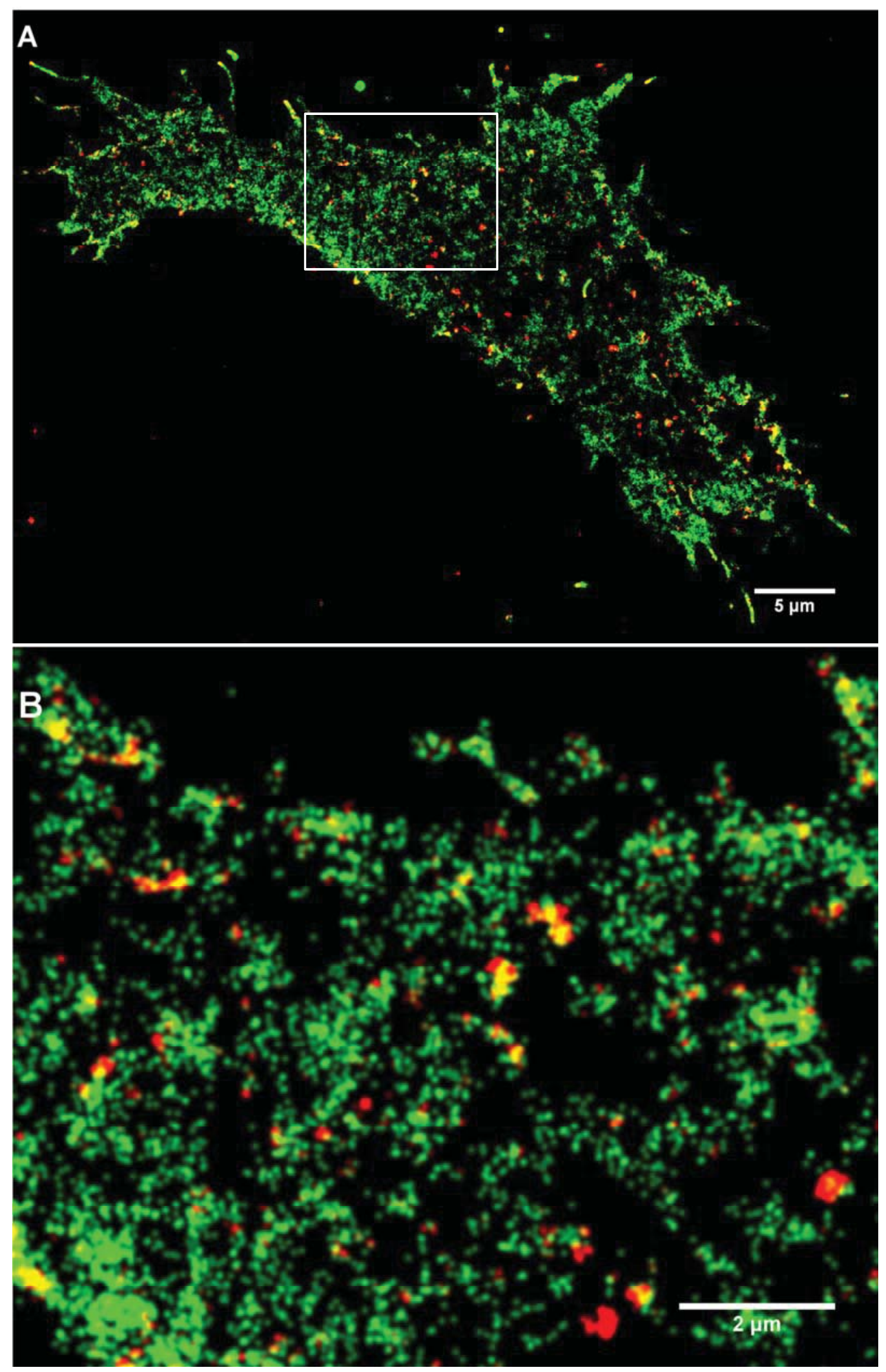

FIGURE S10 Image reconstructions of the distribution of states exhibited by liganded EGF receptor proteins in an MCF7 cell. The resolution of the reconstructed images is $30 \mathrm{~nm} /$ pixel. $(A)$ Image showing the areas travelled by receptors in the fast diffusion state (green), and areas where receptors in the slow diffusion state were detected (red). (B) Zoomed image of the indicated area (white box) in $A$. 

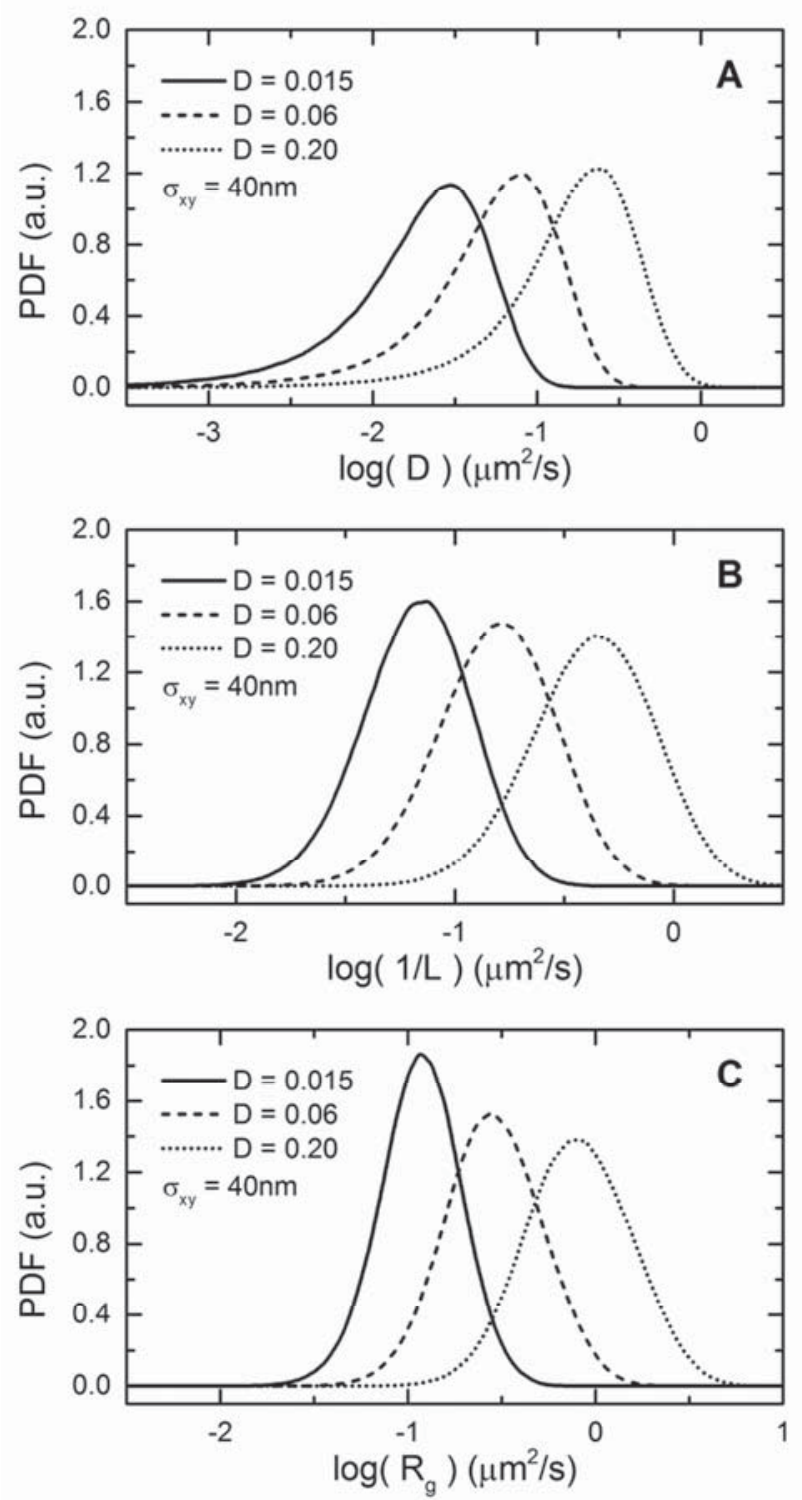

FIGURE S11 Distribution of found quantification measure values for pure one-population diffusion. The histograms of three different diffusion constants are shown, where in all cases we added a localization inaccuracy $\sigma_{x y}$ of $40 \mathrm{~nm}$ to the positions in the simulations. (A) Histograms of values found using a windowed MSD. The broadening in the slower diffusion distributions are due to the convolution with the localization inaccuracy. (B) Histogram of values found using relative confinement. (C) Histogram of values found using gyration. 


\subsection{References}

1. Goulian, M. \& Simon, S. M. Tracking single proteins within cells. Biophys. J. 79, 2188-98 (2000).

2. Sako, Y., Minoghchi, S. \& Yanagida, T. Singlemolecule imaging of EGFR signalling on the surface of living cells. Nat. Cell Biol. 2, 168-72 (2000)

3. Wieser, S. \& Schütz, G. J. Tracking single molecules in the live cell plasma membrane-Do's and Don't's. Methods 46, 131-40 (2008).

4. Sergé, A., Bertaux, N., Rigneault, H. \& Marguet, D. Dynamic multiple-target tracing to probe spatiotemporal cartography of cell membranes. Nat. Methods 5, 687-94 (2008).

5. Holtzer, L. \& Schmidt, T. in Single Particle Tracking and Single Molecule Energy Transfer (Bräuchle, C., Lamb, D. C. \& Michaelis, J.) (WILEY-VCH Verlag $\mathrm{GmbH}, 2010$ ).

6. Low-Nam, S. T. et al. ErbB1 dimerization is promoted by domain co-confinement and stabilized by ligand binding. Nat. Struct. Mol. Biol. 18, 1244-1249 (2011).

7. Lippincott-Schwartz, J., Snapp, E. \& Kenworthy, A. Studying protein dynamics in living cells. Nat. Rev. Mol. Cell Biol. 2, 444-56 (2001).

8. Miyawaki, A. Proteins on the move: insights gained from fluorescent protein technologies. Nat. Rev. Mol. Cell Biol. 12, 656-68 (2011).

9. Giepmans, B. N. G., Adams, S. R., Ellisman, M. H. \& Tsien, R. Y. The fluorescent toolbox for assessing protein location and function. Science 312, 217-24 (2006)

10. Kusumi, A. et al. Paradigm shift of the plasma membrane concept from the two-dimensional continuum fluid to the partitioned fluid: high-speed single-molecule tracking of membrane molecules. Annu. Rev. Biophys. Biomol. Struct. 34, 351-78 (2005)

11. Cambi, A. \& Lidke, D. S. Nanoscale membrane organization: where biochemistry meets advanced microscopy. ACS Chem. Biol. 7, 139-49 (2012).

12. Sako, Y. \& Yanagida, T. Single-molecule visualization in cell biology. Nat. Rev. Mol. Cell Biol. Suppl, SS1-5 (2003)

13. Saxton, M. J. \& Jacobson, K. Single-particle tracking: applications to membrane dynamics. Annu. Rev. Biophys. Biomol. Struct. 26, 373-99 (1997).

14. Engelman, D. M. Membranes are more mosaic than fluid. Nature 438, 578-80 (2005)

15. Shaw, A. S. Lipid rafts: now you see them, now you don't. Nat. Immunol. 7, 1139-42 (2006).

16. Jacobson, K., Mouritsen, O. G. \& Anderson, R. G. W Lipid rafts: at a crossroad between cell biology and physics. Nat. Cell Biol. 9, 7-14 (2007).

17. Citri, A. \& Yarden, Y. EGF-ERBB signalling: towards the systems level. Nat. Rev. Mol. Cell Biol. 7, 505-16 (2006).

18. Lemmon, M. A. \& Schlessinger, J. Cell signaling by receptor tyrosine kinases. Cell 141, 1117-34 (2010).

19. Kapanidis, A. N. \& Strick, T. Biology, one molecule at a time. Trends Biochem. Sci. 34, 234-43 (2009).

20. Schütz, G. J., Schindler, H. \& Schmidt, T. Singlemolecule microscopy on model membranes reveals anomalous diffusion. Biophys. J. 73, 1073-80 (1997).

21. Brameshuber, M. \& Schütz, G. J. in Springer Series on Fluorescence 1-37 (Springer Berlin Heidelberg, 2012). doi:10.1007/4243 2011 38
22. Dietrich, C., Yang, B., Fujiwara, T., Kusumi, A. \& Jacobson, K. Relationship of lipid rafts to transient confinement zones detected by single particle tracking. Biophys. J. 82, 274-84 (2002).

23. De Keijzer, S. et al. A spatially restricted increase in receptor mobility is involved in directional sensing during Dictyostelium discoideum chemotaxis. J. Cell Sci. 121, 1750-7 (2008).

24. Cebecauer, M., Spitaler, M., Sergé, A. \& Magee, A. I. Signalling complexes and clusters: functional advantages and methodological hurdles. J. Cell Sci. 123, 309-20 (2010).

25. Harding, A. S. \& Hancock, J. F. Using plasma membrane nanoclusters to build better signaling circuits. Trends Cell Biol. 18, 364-371 (2008).

26. Harding, A. Ras nanoclusters: combining digital and analog signaling. Cell Cycle 127-134 (2008).

27. Kholodenko, B. N., Hancock, J. F. \& Kolch, W. Signalling ballet in space and time. Nat. Rev. Mol. Cell Biol. 11, 414-426 (2010).

28. Radhakrishnan, K., Halász, Á., McCabe, M. M. Edwards, J. S. \& Wilson, B. S. Mathematical simulation of membrane protein clustering for efficient signal transduction. Ann. Biomed. Eng. 40, 2307-18 (2012).

29. Xiao, Z., Zhang, W., Yang, Y., Xu, L. \& Fang, X Single-molecule diffusion study of activated EGFR implicates its endocytic pathway. Biochem. Biophys. Res. Commun. 369, 730-4 (2008)

30. Rong, G. \& Reinhard, B. M. Monitoring the size and lateral dynamics of ErbB1 enriched membrane domains through live cell plasmon coupling microscopy. PLoS One 7, e34175 (2012).

31. Orr, G. et al. Cholesterol dictates the freedom of EGF receptors and HER2 in the plane of the membrane. Biophys. J. 89, 1362-73 (2005).

32. Manley, S. et al. High-density mapping of singlemolecule trajectories with photoactivated localization microscopy. Nat. Methods 5, 155-157 (2008).

33. Kusumi, a, Sako, Y. \& Yamamoto, M. Confined lateral diffusion of membrane receptors as studied by single particle tracking (nanovid microscopy). Effects of calcium-induced differentiation in cultured epithelial cells. Biophys. J. 65, 2021-40 (1993).

34. Michalet, X. Mean square displacement analysis of single-particle trajectories with localization error: Brownian motion in an isotropic medium. Phys. Rev. E 82, 041914 (2010).

35. Simson, R., Sheets, E. D. \& Jacobson, K. Detection of temporary lateral confinement of membrane proteins using single-particle tracking analysis. Biophys. J. 69, 989-93 (1995).

36. Meilhac, N., Le Guyader, L., Salomé, L. \& Destainville, N. Detection of confinement and jumps in single-molecule membrane trajectories. Phys. Rev. E 73, 011915 (2006).

37. Elliott, L. C. C., Barhoum, M., Harris, J. M. \& Bohn, P. W. Trajectory analysis of single molecules exhibiting non-brownian motion. Phys. Chem. Chem. Phys. 13, 4326-34 (2011).

38. Wieser, S., Axmann, M. \& Schütz, G. J. Versatile analysis of single-molecule tracking data by comprehensive testing against Monte Carlo simulations. Biophys. J. 95, 5988-6001 (2008).

39. Helmuth, J. a, Burckhardt, C. J., Koumoutsakos, P., Greber, U. F. \& Sbalzarini, I. F. A novel supervised 
trajectory segmentation algorithm identifies distinct types of human adenovirus motion in host cells. $J$. Struct. Biol. 159, 347-58 (2007).

40. Persson, F., Lindén, M., Unoson, C. \& Elf, J. Extracting intracellular diffusive states and transition rates from single-molecule tracking data. Nat. Methods 10, 265-9 (2013).

41. Jaqaman, K. et al. Cytoskeletal Control of CD36 Diffusion Promotes Its Receptor and Signaling Function. Cell 146, 593-606 (2011).

42. Bouzigues, C. \& Dahan, M. Transient directed motions of $\operatorname{GABA}(A)$ receptors in growth cones detected by a speed correlation index. Biophys. J. 92, 654-60 (2007).

43. Montiel, D., Cang, H. \& Yang, H. Quantitative characterization of changes in dynamical behavior for single-particle tracking studies. J. Phys. Chem. B 110, 19763-70 (2006).

44. Ott, M., Shai, Y. \& Haran, G. Single-particle tracking reveals switching of the HIV fusion peptide between two diffusive modes in membranes. J. Phys. Chem. B 117, 13308-21 (2013).
45. Eddy, S. R. What is Bayesian statistics? Nat. Biotechnol. 22, 1177-8 (2004).

46. Berglund, A. Statistics of camera-based singleparticle tracking. Phys. Rev. E 82, 011917 (2010).

47. Smith, C. S., Joseph, N., Rieger, B. \& Lidke, K. A. Fast, single-molecule localization that achieves theoretically minimum uncertainty. Nat. Methods 7, 373-5 (2010).

48. Lidke, D. S. et al. Quantum dot ligands provide new insights into erbB/HER receptor-mediated signal transduction. Nat. Biotechnol. 22, 198-203 (2004).

49. Arndt-Jovin, D. Quantum dots shed light on processes in living cells. SPIE Newsroom 2-4 (2006). doi:10.1117/2.1200605.0228

50. Semrau, S. \& Schmidt, T. Particle image correlation spectroscopy (PICS): retrieving nanometer-scale correlations from high-density single-molecule position data. Biophys. J. 92, 613-21 (2007).

51. Semrau, S., Holtzer, L., González-Gaitán, M. \& Schmidt, T. Quantification of Biological Interactions with Particle Image Cross-Correlation Spectroscopy (PICCS). Biophys. J. 100, 1810-8 (2011). 



\title{
Chapter 4
}

\section{Evaluation of fluorophores to label SNAP-tag fused proteins for multicolor single molecule tracking microscopy in live cells}

\author{
Peter J. Bosch ${ }^{1}$ \\ Ivan R. Corrêa Jr. ${ }^{2}$ \\ Michael H. Sonntag ${ }^{3}$ \\ Luc Brunsveld ${ }^{3}$ \\ Johannes S. Kanger ${ }^{1}$ \\ Vinod Subramaniam ${ }^{1,4}$
}

1. Nanobiophysics, MESA+ Institute for Nanotechnology and MIRA Institute for Biomedical Technology and Technical Medicine, University of Twente, PO Box 217, 7500AE Enschede, The Netherlands

2. New England Biolabs, Ipswich, MA, USA

3. Laboratory of Chemical Biology, Department of Biomedical Engineering, and Institute of Complex Molecular Systems, Eindhoven University of Technology, The Netherlands

4. Present address: FOM Institute AMOLF, Science Park 104, 1098 XG Amsterdam, The Netherlands 


\begin{abstract}
Single molecule tracking has become a widely used technique to study protein dynamics and their organization in the complex environment of the cell. In particular, the spatiotemporal distribution of membrane receptors is an active field of study due to its putative role in the regulation of signal transduction. The SNAP-tag is an intrinsically monovalent and highly specific genetic tag for attaching a fluorescent label to a protein of interest. Little information is currently available on the choice of optimal fluorescent dyes for single molecule microscopy utilizing the SNAP-tag labeling system.

We surveyed 6 green and 16 red excitable dyes for their suitability in single molecule microscopy of SNAP-tag fusion proteins in live cells. We determined the nonspecific binding levels and the photostability of these dye conjugates when bound to a SNAP-tag fused membrane protein in live cells. We found that only a limited subset of the dyes tested is suitable for single molecule tracking microscopy.

The results show that a careful choice of the dye to conjugate to the SNAP-substrate to label SNAPtag fusion proteins is very important, as many dyes either suffer from rapid photobleaching or high nonspecific staining. These characteristics appear to be unpredictable beforehand, which motivated the need to perform the systematic survey presented here. We have developed a protocol for evaluating the best dyes, and for the conditions that we evaluated, we find that Dy 549 and CF 640 are the best choices tested for single molecule tracking. Using an optimal dye pair, we also demonstrate the possibility of dual-color single molecule imaging of SNAP-tag fusion proteins. This survey provides an overview of the photophysical and imaging properties of a range of SNAP-tag fluorescent substrates, enabling the selection of optimal dyes and conditions for single molecule imaging of SNAP-tagged fusion proteins in eukaryotic cell lines.
\end{abstract}




\subsection{Introduction}

Single molecule fluorescence microscopy has emerged in recent years as a powerful tool to investigate the structural dynamics and biological functions of proteins and macromolecular protein complexes ${ }^{1-5}$. Single molecule fluorescence approaches can reveal the dynamic interactions of individual proteins and heterogeneity in the spatial distribution of proteins that are difficult to detect using other fluorescence microscopy approaches ${ }^{6-8}$. Despite the extraordinary advances in single molecule fluorescence achieved to date, there remain many technical challenges that must be overcome to systematically study proteins in their native highly complex cellular environment. One of the challenges involves the specific and monovalent labeling of proteins of interest with a photostable fluorescent probe. In the last decade several technologies have been developed that permit proteins to be specifically tagged with organic dyes in live cells ${ }^{2,3,9-11}$. In this paper we focus on the fluorescent labeling of proteins for single molecule tracking.

Single molecule fluorescence microscopy allows the tracking of proteins in a living cell at high resolution for a short period of time $\mathrm{e}^{12-15}$. The trajectories obtained contain valuable spatiotemporal information on interactions of proteins with their microenvironment ${ }^{16-18}$. For instance, a protein may interact with other molecules resulting in transient slowed diffusion or confinement by the cytoskeletal or other nanoscale compartmentalization structures in the plasma membrane ${ }^{11,15,19-23}$. One of the main advantages of single molecule fluorescence microscopy is the ability to track single protein molecules to provide details on the kinetics of protein association and dissociation. When the trajectories of a single protein species are recorded in multiple colors, they can reveal the kinetics of homo-dimerization interactions by co-movement of the labeled molecules ${ }^{11,24}$. For this co-movement analysis, the protein species needs to be labeled with fluorophores emitting light at spectrally distinct wavelengths to allow simultaneous visualization at high resolution of two distinct proteins (of one protein species). Knowledge of protein interactions and their kinetics is important to understand the underlying signal transduction mechanisms and to model the cellular signal regulatory system ${ }^{25-27}$.

A common approach to fluorescently label proteins is by cloning and expressing the protein of interest fused to an autofluorescent protein (FP). Several FPs are currently available that are suitable for single molecule tracking, such as mCitrine, $\mathrm{mCherry}^{28}$, and the infra-red iRFP ${ }^{29}$. While these genetically encoded labels allow multi-color tracking, FPs cannot match the photostability of small organic dyes ${ }^{2,30}$, limiting the timescale over which a protein can be tracked, and the accuracy with which it can be localized. To permit imaging of longer trajectories, fluorescent probes should ideally be bright and photostable (i.e. slow to photobleach) in addition to being specifically linkable to the protein of interest. The tools of choice in this case are organic dyes and quantum dots (Qdots). Although Qdots are extremely bright and photostable, they are larger than most proteins themselves, which might sterically hinder the movement of the protein ${ }^{31}$. Additionally, the use of monovalent quantum dots requires custom fabrication ${ }^{32-34}$, and they might suffer from nonspecific labeling or aggregation ${ }^{35,36}$.

More recent genetic techniques allow the specific and monovalent labeling of recombinant proteins with small organic fluorophores in live cells. Labeling by means of protein tags complements other approaches to label proteins with organic fluorophores such as labeling with fluorescently labeled ligands and immunostaining with antibodies or Fab fragments. Since the tag does not compete with ligand binding and has no antagonistic function, the effects of ligands or inhibitors on ligand-free and fully functional receptors can be studied. The monovalency of the substrates ensures that no artificial clustering is induced. Among the most versatile of the protein tags is the SNAP-tag, a $20 \mathrm{kDa}$ mutant of the human DNA repair protein $\mathrm{O}^{6}$-alkylguanine-DNA alkyltransferase (hAGT) that reacts specifically and rapidly with benzylguanine (BG) or benzylchloropyrimidine $(\mathrm{CP})$ derivatives, leading to a covalent labeling of the SNAP-tag with a synthetic probe ${ }^{37-41}$. The reaction occurs through a well-defined mechanism and predictable 
monovalent stoichiometry. For example, BG substrates derived from organic fluorophores react with SNAP-tag to provide specific labeling of a protein species with a fluorescent label at physiologically conditions in the cell (Fig. 4.1). For labeling at the single molecule level, SNAP-tag is especially suitable to label plasma membrane resident proteins using membrane impermeable substrates.
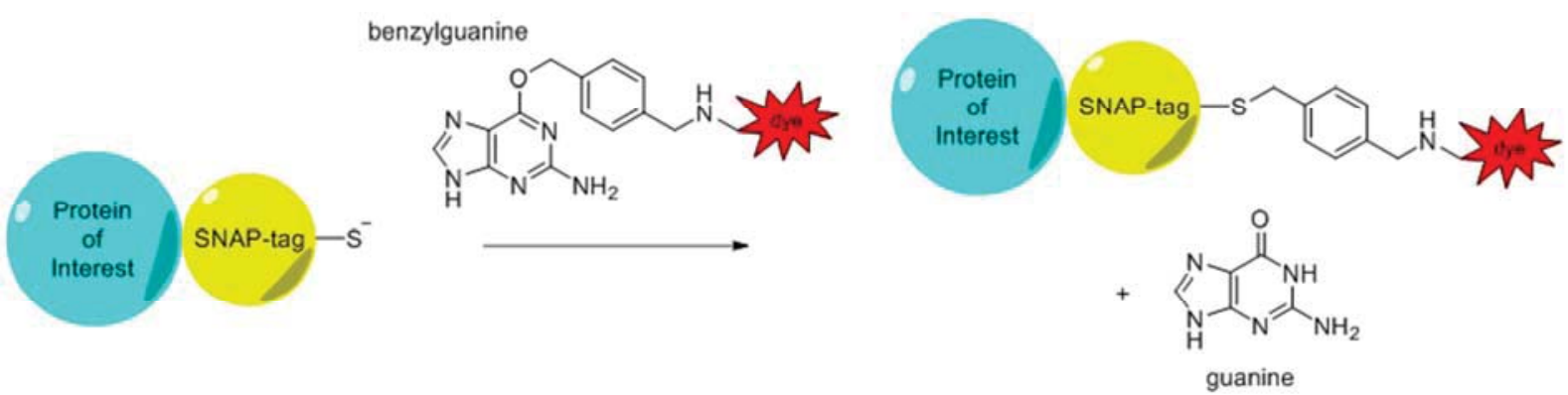

FIGURE 4.1 Schematic of the binding of a benzylguanine (BG) substrate to a SNAP-tag fusion protein. The SNAP-tag is fused to a protein of interest. Upon binding, the benzyl group reacts with a cysteine in the active site of SNAP-tag, releasing the guanine group. In this survey the BG was conjugated to fluorescent dyes, but BG can in principle be coupled to any molecule of choice.

There is a wide variety of reported applications in protein labeling for the SNAP-tag system, including super-resolution imaging ${ }^{42-44}$, analysis of protein function ${ }^{45}$ and protein half-life ${ }^{46}$, protein-protein interactions ${ }^{47}$, sensing cell metabolites ${ }^{48}$, and for the identification of drug targets ${ }^{49}$. SNAP-tag labeling has also been utilized to study several protein complexes at the single molecule level ${ }^{50-59}$. Although the application of the SNAP-tag labeling system for single molecule tracking had already been suggested ${ }^{14}$, only recently have Calebiro et al. demonstrated the first example (to the best of our knowledge) of this tagging technology for single color fluorescence tracking ${ }^{60}$. These authors used direct receptor labeling with SNAP-tag to dynamically monitor the adrenergic receptors $\beta 1 \mathrm{AR}$ and $\beta 2 \mathrm{AR}$ and the $\gamma$-aminobutyric acid GABAB receptor on intact cells, and compared their spatial arrangement, mobility, and supramolecular organization. Benke et al. have recently shown a new approach to single molecule tracking by using the blinking properties of synthetic dyes attached to SNAP-tag ${ }^{61}$. While this approach optimizes the total number of observable diffusion steps, it does not facilitate the observation of a single protein for as long as possible as required to study binding associations.

Here we report a comprehensive survey on the photostability and binding specificity of several SNAP-tag fluorescent substrates using widefield and total internal reflection fluorescence (TIRF) single molecule microscopy. We have investigated the suitability of 22 fluorescent substrates (BGdyes) by characterizing their properties in living cells using a C-terminal (extracellular) SNAP-tag fusion to the epidermal growth factor receptor (EGFR), a plasma membrane resident protein. Due to the significant autofluorescence of cells when using blue excitable dyes, we limited our survey to green- and red-excitable dyes. Since a lot of dyes are available in this spectral range, we selected dyes from different manufacturers which are commonly available and used, and tried to include dyes from various chromophore families. We emphasize that it is essential to study the fluorophores in the cellular setting, because photophysical properties are known to differ depending on the nature of their conjugate and its microenvironment. For example, different photostabilities have been observed for fluorescent proteins on different interfaces, due to the apparent role of the protein shell rigidity for each chromophore ${ }^{62}$. In addition, the fluorescence of a number of fluorophores may be quenched by electron donors like guanine, tryptophan, etc. ${ }^{63}$. Therefore the photophysical properties of free substrates in solution or immobilized on a glass surface do not necessarily reflect their properties after reaction with the SNAP-tag fused protein. 
Very recently, the photostability of two red-excitable fluorescent substrates was measured for another protein tag (A-TMP) at the single molecule level ${ }^{64}$. The binding specificity for these substrates was not determined.

\subsection{Methods}

\subsubsection{Chemicals, Purification and Analysis of SNAP-tag substrates}

Commercially available compounds were used without further purification. SNAP-Surface Alexa Fluor 546 (BG-Alexa 546), SNAP-Surface 549 (BG-Dy 549), SNAP-Surface 632 (BG-Dy 632), SNAP-Surface 647 (BG-Dy 647), SNAP-Surface Alexa Fluor 647 (BG-Alexa 647), SNAP-Surface 649 (BG-Dy 649) were obtained from New England Biolabs, Inc.

BG-Atto 550, BG-Atto 565, BG-Atto 620, BG-Atto 633, BG-Atto 647N, BG-Atto 655, and BGTF5 have been described previously ${ }^{40-42,65}$. The remaining substrates for the labeling of SNAP-tag fusion proteins were prepared by reacting the building block $\mathrm{BG}-\mathrm{NH}_{2}$ (S9148, New England Biolabs, Inc.) with commercially available $N$-hydroxysuccinimide esters (NHS) of the corresponding fluorophores. Atto Rho6G and Atto 532 were obtained from Atto-Tec GmbH (Siegen, Germany); Dy 549, Dy 630, Dy 634, Dy 648, and Dy 651 were obtained from Dyomics GmbH (Jena, Germany). CF633 and CF640R were obtained from Biotium Inc. (Hayward, CA, USA), and Star635 was obtained from Abberior GmbH (Göttingen, Germany).

BG-549-549, BG-Dy 651, BG-CF 633, BG-CF 640R, and BG-Star 635 were purified and analyzed with the following equipment. Reversed-phase high-performance liquid chromatography (HPLC) was performed on Agilent LC/MS Single Quad System 1200 Series (analytical) and Agilent 1100 Preparative-scale Purification System (semi-preparative). Analytical HPLC was performed on Waters Atlantis T3 C18 column $(2.1 \times 150 \mathrm{~mm}, 5 \mu \mathrm{m}$ particle size $)$ at a flow rate of $0.5 \mathrm{~mL} / \mathrm{min}$ with a binary gradient from Phase A (0.1 M triethyl ammonium bicarbonate (TEAB) or $0.1 \%$ trifluoroacetic acid (TFA) in water) to Phase B (acetonitrile) and monitored by absorbance at $280 \mathrm{~nm}$. Semi-preparative HPLC was performed on VYDAC 218TP series C18 polymeric reversed-phase column $(22 \times 250 \mathrm{~mm}, 10 \mu \mathrm{m}$ particle size $)$ at a flow rate of $20 \mathrm{~mL} / \mathrm{min}$. Mass spectra were recorded by electrospray ionization (ESI) on an Agilent 6120 Quadrupole LC/MS system. BG-Atto Rho6G, BG-Dy 630, BG-Dy 634, and BG-Dy 648 were purified and analyzed with the following equipment. Reversed-phase high-performance liquid chromatography (HPLC) was performed on Shimadzu SCL-10 AD VP series (analytical) and Shimadzu LC-20 AD System (preparative). Analytical HPLC was performed on a reversed phase HPLC column (GraceSmart PP18, $50 \mathrm{~mm} \times 2.1 \mathrm{~mm}, 3 \mu \mathrm{m}$ ) at a flow rate of $0.20 \mathrm{~mL} / \mathrm{min}$ and a binary gradient of acetonitrile in water (both containing $0.1 \%$ formic acid) at $298 \mathrm{~K}$. Mass and UV-VIS spectra were recorded with an Ion-Trap (LCQ Fleet Ion Trap Mass Spectrometer, Thermo Scientific) and diode array detector (Finnigan Surveyor PDA Plus detector, Thermo Electron Corporation). Preparative reversed-phase HPLC was performed on a reversed phase HPLC column (GraceAlpha C18 5u, $250 \mathrm{~mm} \times 4.6 \mathrm{~mm})$ at a flow rate of $1 \mathrm{~mL} / \mathrm{min}$ with an isocratic gradient of Phase A $(0.1 \%$ formic acid in water or $25 \mathrm{mM}$ ammonium acetate in water, $\mathrm{pH} 4)$ to Phase $\mathrm{B}(0.1 \%$ formic acid in acetonitrile) and monitored with a UV-VIS detector (SPD-10AV VP series, Shimadzu).

\subsubsection{General Procedure for the Synthesis of SNAP-tag substrates}

For BG-Dy 651, BG-CF633, BG-CF640R and BG-Star 635, reactions were performed at $1-2 \mu \mathrm{mol}$ scale using 1.0 equiv of $\mathrm{BG}-\mathrm{NH}_{2}$ in anhydrous $N, N$-dimethylformamide (DMF) in the presence of triethylamine (2.0 equiv), and the corresponding fluorophore $\mathrm{N}$-hydroxysuccinimidyl ester (1.0 equiv), at room temperature. For BG-Atto Rho6G, BG-Dy 630, BG-Dy 634, and BG-Dy 648, reactions were performed at $0.1 \mu \mathrm{mol}$ scale using 1.0 equiv of $\mathrm{BG}-\mathrm{NH}_{2}, 1 \%$ triethylamine, and 3.0 - 4.0 equiv of the fluorophore $\mathrm{N}$-hydroxysuccinimidyl ester, at $30^{\circ} \mathrm{C}$. BG-Dy 549-549 (BG-549- 
549) was synthesized by reacting the bifunctional building block BG-Lys(Dde)-Fmoc ${ }^{66}$ with a $2 \%$ solution of hydrazine in DMF $(0.5 \mathrm{~mL})$, followed by solvent removal under high vacuum overnight, and treatment with 2.2 equiv of triethylamine and 2.2 equiv of Dy549 Nhydroxysuccinimidyl ester at room temperature. All coupling reactions were carried out for $12 \mathrm{~h}$. Reaction completion was monitored by HPLC. Reaction mixtures were concentrated and purified by reversed-phase HPLC. Each substrate was analyzed by mass spectrometry and UV absorption. Isolated yields are given in parentheses when determined and are not optimized. BG-CF633 (54\%): ESI-MS $m / z 1074.4[\mathrm{M}+\mathrm{H}]^{+}, \lambda_{\max }=630 \mathrm{~nm}$; BG-CF640R (80\%): ESI-MS $m / z 1083.3[\mathrm{M}-\mathrm{H}]^{-}$, $\lambda_{\max }=642 \mathrm{~nm}$; BG-Star635 (30\%): ESI-MS m/z $1148.3[\mathrm{M}+\mathrm{H}]^{+}, \lambda_{\max }=631 \mathrm{~nm}$; BG-651 (35\%): ESIMS $m / z 1019.3[\mathrm{M}+\mathrm{H}]^{+}, \lambda_{\max }=656 \mathrm{~nm}$; BG-AttoRho6G: ESI-MS $m / z 766.6[\mathrm{M}+\mathrm{H}]^{+}, \lambda_{\max }=535 \mathrm{~nm}$; BG-Dy630: ESI-MS $m / z 887.6[\mathrm{M}+\mathrm{H}]^{+}, \lambda_{\max }=631 \mathrm{~nm}$, BG-Dy634: ESI-MS $m / z 1155.5[\mathrm{M}+\mathrm{H}]^{+}$, $\lambda_{\max }=631 \mathrm{~nm}$, BG-Dy648: ESI-MS $m / z 989.5[\mathrm{M}+\mathrm{H}]^{+}, \lambda_{\max }=650 \mathrm{~nm}$; BG-Dy 549x2 (61\%): ESI-MS $m / z 998.8[\mathrm{M}-2 \mathrm{H}]^{2-}, \lambda_{\max }=557 \mathrm{~nm}$.

\subsubsection{Cell culture}

All cell culture materials were obtained from PAA Laboratories (Pasching, Austria) unless stated otherwise. The MCF7 epithelial human breast cancer cell line, and plasmids coding for SNAPEGFR were a gift from Jenny Ibach (Max Planck Institute, Dortmund, Germany). MCF7 cells were cultured in high glucose Dulbecco's Modified Eagle's medium (DMEM) supplemented with $10 \% \mathrm{FBS}$ and Pen Strep at $37^{\circ} \mathrm{C}$ with 5\% CO2. The $\mathrm{H} 441$ epithelial human lung adenocarcinoma cancer cell line was a gift from Anton Terwisscha van Scheltinga (Department of Medical Oncology, University of Groningen, The Netherlands). These cells were cultured in Roswell Park Memorial Institute (RPMI) 1640 medium supplemented as above. The HeLa epithelial human cervix adenocarcinoma cancer cell line was a gift from Wilma Petersen (University of Twente, The Netherlands), and was cultured in Iscove's Modified Dulbecco's Media (IMDM) supplemented as above.

We created stably expressing SNAP-EGFR HeLa cells by transfecting HeLa cells in a $60 \mathrm{~cm}^{2}$ well of $40 \%$-confluent cells using $9 \mu \mathrm{g}$ of SNAP-EGFR plasmid DNA and $20 \mu \mathrm{L}$ Lipofectamine LTX and $9 \mu \mathrm{L}$ Plus reagent (Invitrogen) in $15 \mathrm{~mL}$ Pen Strep free cell medium, as described in the suppliers' protocol. Selection ( $1400 \mu \mathrm{g} / \mathrm{mL}$ of active G418) was applied after $24 \mathrm{~h}$. After 5 days the cells were split over two 6-well plates. After 10 days the wells were screened for expression of SNAP-EGFR by labeling with $500 \mathrm{nM}$ BG-Alexa 546 for $15 \mathrm{~min}$ and fluorescence microscopy analysis on the single molecule sensitive microscope as described later. Note that the expression level can be very low at this stage, and the imaging required a single molecule sensitive fluorescence microscope. One well contained positive cells with an expression level slightly above the single molecule level; these cells were further cultured. For culturing, the concentration of active $\mathrm{G} 418$ was $350 \mu \mathrm{g} / \mathrm{mL}$.

\subsubsection{Sample Preparation}

For each dye, video recordings were taken of four samples: the SNAP-tag positive cells and the three negative cell lines. Before measurements, HeLa cells stably expressing SNAP-EGFR, HeLa cells, MCF7 cells, and H441 cells were plated in Greiner Bio CellView dishes (product \#627870) in full medium, and left overnight to allow the cells to adhere to the glass. The HeLa cells stably expressing SNAP-EGFR were also starved in FBS free medium and left for another night to reduce the activity and internalization of the EGFR fusion protein. On the day of the experiment, cells were washed with starvation medium containing 0.5\% BSA. Labeling of the SNAP-EGFR proteins was carried out thereafter by incubating the cells for 2 minutes ( \pm 10 seconds) with $400 \mathrm{nM}$ of each BG-dye in starvation medium containing $0.5 \%$ BSA. Samples were washed immediately by replacing the labeling solution with PBS buffer supplemented with magnesium and calcium. This 
washing step was repeated at least three times. Incubation and washing of the SNAP-tag negative cells with the substrates was performed using the same conditions.

\subsubsection{Microscope hardware}

Measurements were performed on an inverted microscope with an Olympus PlanApo 100x/1,45 Oil TIRF objective. For the excitation we used a $532 \mathrm{~nm} 400 \mathrm{~mW}$ solid state laser from Pegasus Shanghai Optical Systems (Pegasus Lasersysteme, Wallenhorst, Germany), and a $637 \mathrm{~nm} 300 \mathrm{~mW}$ diode laser from Changchun New Industries Optoelectronics (Laser 2000, Vinkeveen, The Netherlands). The infrared light produced by the lasers was not sufficiently suppressed; we therefore filtered the green laser light with an FF01-543/22 (SemRock, Rochester, NY) filter and the red laser light with a D644/10m (Chroma, Olching, Germany) bandpass filter. The two laser beams were combined with a $610 \mathrm{~nm}$ longpass mirror. An achromatic quarter wave plate (VMTIM Optomechanische Werke, Jena, Germany) was used to obtain circularly polarized excitation light. An FF494/540/650-Di01 (SemRock) dichroic mirror separated the excitation light from the emission light. The optical path and filters reduced the power of the laser light reaching the objective. For the green laser $88 \mathrm{~mW}$ was measured after the objective, and for the red laser 47 $\mathrm{mW}$ was measured after the objective. The excitation field of view had an approximate diameter of $60 \mu \mathrm{m}$, which resulted in an estimated 3.1 and $1.7 \mathrm{~kW} / \mathrm{cm} 2$ for the green and red excitation intensity respectively. The emission light was filtered with an NF03-532/1064E (SemRock) and an NF02-633S (SemRock) notch filter, and then split with an FF640-FDi01 (SemRock) imaging flatness quality dichroic mirror. The emission light from the green excitation dyes was passed through a FF01-580/60 (SemRock) bandpass filter, and the light from the red excitation dyes through a HQ680/60m (Chroma) bandpass filter. The images were acquired using two Andor iXon EM+ DU-897 back illuminated EMCCD cameras. Using a calibration sample with grid lines separated at known distances, we determined that the pixel size was $119 \pm 2 \mathrm{~nm}$ (the magnification was $134 \mathrm{x}$ ). The sample was only illuminated during the acquisition time by utilizing the TTL output of the camera and TTL input of the lasers. Samples were heated to $35-37^{\circ} \mathrm{C}$ on the microscope with a stage heating plate and an objective ring heater.

\subsubsection{Microscopy settings}

Measurements to determine nonspecific binding of the SNAP substrates were performed using widefield and TIRF illumination. Measurements to determine the photobleaching of the substrates were performed using widefield illumination. The illumination time differed for each fluorescent substrate, and was chosen in such a way that single molecules were clearly visible over the autofluorescence background of the cell. We sought to collect the same average number of photons per molecule in a frame for each fluorescent substrate. Since the quantum yield has not been previously determined for all fluorescent substrates, the illumination time to yield an equal number of emitted photons per molecule could not be calculated beforehand. Videos were recorded at 20-30 fps, which is the highest allowed frame rate of the camera at the maximum readout rate of $10 \mathrm{MHz}$ and frame size of $512 \times 512$ pixels. Each video recording consisted of 800 frames. Before recording each video, a minimal number of frames (approximately 10 to 30 frames) were used to focus on the basal membrane of the cell.

\subsubsection{Single molecule brightness}

To allow conversion of pixel counts to photons, a calibration of the gain of the two EMCCD cameras was performed by the mean-variance method (Fig. S1). The slope of the line in this curve is equal to the inverse gain of the camera. A gain of $49.9 \pm 0.1$ was found for the camera recording the green-excitable dyes, and a gain of $32.5 \pm 0.1$ was found for the camera recording the redexcitable dyes. The pixel intensities in counts are divided by the camera gain to convert the pixel 
intensities to photons. The brightness of one molecule (sometimes also termed spot intensity) was calculated as the integrated intensity of a single molecule using a Gaussian fit performed by the tracking algorithm used ${ }^{67}$. This yields for all single molecules the number of detected photons per single molecule per frame. We defined the single molecule brightness $B$ as the average of these numbers in one recording.

The brightness of the dye conjugates can be compared between dyes by a relative brightness (Table S1), which is a normalized value given by the single molecule brightness $B$ (Fig. S2) divided by the acquisition time, the excitation efficiency $\eta_{e}$, the emission efficiency, and by the laser excitation power. The excitation efficiency $\eta_{e}$ is equal to the fraction of the maximum value of the excitation spectrum of the dye at the wavelength of the lasers, i.e. $532 \mathrm{~nm}$ for the green dyes, and $637 \mathrm{~nm}$ for the red dyes. The spectra of the dyes were downloaded from the SemRock website (http://www.semrock.com), except for the CF dyes, TF5 and Star635; we measured the spectra for these dyes with a Varian Cary Eclipse fluorescence spectrophotometer (Palo Alto, CA).

\subsubsection{Tracking of single molecules}

To obtain trajectories from the raw videos, we used previously described tracking software ${ }^{11,67}$. The settings used for the cost matrices in this software can be found in the appendix of Chapter 2 . For the initial detection of molecules the tracking algorithm uses an intensity threshold. This threshold was taken the same for all video series of one fluorescent substrate to obtain a fair comparison of the level of specific and nonspecific labeling. The threshold was determined in the situation where the substrates are incubated with SNAP-tag expressing cells (specifically attached); we used the same threshold values in the detection of nonspecifically attached substrates.

After obtaining the single molecule trajectories, two filtering operations were applied with the purpose of discarding very short tracks, and differentiating between completely immobile and (transiently) mobile molecules. Very short tracks (having less than 7 localizations in total) were excluded as they did not contain much significant information; there is also a higher chance that a fluorescent spot that is detected only for a few frames was attributable to noise rather than to a specifically labeled fluorescent molecule. A segment of a track was defined as the subsequent positions of a fluorescent molecule in adjacent frames. This meant that blinking of a dye resulted in multiple segments within a track. Immobile tracks were discarded because they often represented dye molecules bound to the glass surface, and were detected using a radius of gyration algorithm (manuscript in preparation ${ }^{68}$. The threshold for the trajectory area was defined by a gyration radius of $40 \mathrm{~nm}$, as this corresponded to the apparent area travelled by an immobile molecule due to the localization accuracy.

\subsubsection{Analysis of single molecule photobleaching rate}

The number of fluorescent molecules $N(i)$ in each frame $i$ was determined for each recording. Since photobleaching follows an exponential decay profile, the photobleaching rates are obtained for each video recording by fitting the number of molecules over time with a one component exponential function without offset:

$$
N(i)=N(1) \cdot \exp (-1 / \tau \cdot i)
$$

where $i$ is the framenumber, $\tau$ is the mean photobleaching time (in frames), hence $1 / \tau$ is the rate of photobleaching per frame, and $N(1)$ the fitted number of molecules in this first frame $(\mathrm{i}=1)$. The fit was performed over framenumbers 20 till 600. In the first few frames the autofluorescence of the cells might obscure a proper detection of single molecules by the algorithm. Because the autofluorescence bleaches rapidly, the fluorescent molecules can be reliably detected after 20 
frames. At frame 600 the number of molecules were reduced to a basal level in most recordings. The fluorophore's mean photobleaching time $\tau$ is multiplied by the single molecule brightness $B$. This yields the expected average number of detected photons per molecule $P$.

Since the dye conjugates have different emission spectra, we corrected for the transmission efficiency of the filter set to obtain a precise comparison of the dyes. The most relevant parameter to compare is the photobleaching rate per emitted photon and not per detected photon. This is because not all the emitted photons pass the filters placed before the camera. Not all the emitted photons are collected by the objective, but the fraction of photons collected is the same for all the dyes, and it is therefore not necessary to correct for this. Furthermore the quantum efficiency of the CCD chip is similar around the measured wavelengths. Therefore the photobleaching rates were only corrected for the efficiency of the filter set $\eta_{f}$, which describes the efficiency with which the emitted fluorescence passed the filter set used. The expected number of detectable photons $P_{\text {corr }}$ is given by the expected detected number of photons $P$ divided by the detection efficiency $\eta_{f}$. The detection efficiency $\eta_{f}$ of a dye was determined by integration over the combined transmission spectrum of the dichroic mirrors and the emission filter multiplied by the normalized emission spectrum of the dye. This efficiency is listed for each dye in Table S1 in the Supporting Information. The expected number of detectable photons per molecule $P_{\text {corr }}$ was calculated as:

$$
P_{\text {corr }}=\frac{1}{\eta_{f}} \cdot B \cdot \tau
$$

\subsection{Results}

\subsubsection{Nonspecific binding of the SNAP substrates}

We first screened the dyes to assess the level of nonspecific staining of the dye conjugates in cells not expressing the SNAP-tag fusion protein (SNAP-tag negative cells). We excluded substrates leading to high nonspecific staining of intracellular structures from further analyses. The results from microscopy video recordings of $\mathrm{H} 441$ cells incubated with each dye conjugate are shown in Fig. 4.2 (widefield illumination), and in Fig. S3 (TIRF illumination). In all the images there was full confluence of cells in the field of view. While TIRF images are often preferred over widefield images to record receptor proteins due to the reduced background level, likewise, only nonspecific staining near and at the plasma membrane of the cells will be observed with TIRF imaging. Nonspecifically stained intracellular structures were better observed using widefield imaging, and were used for screening of nonspecific staining. The screening for nonspecific binding was based on observations of at least 50 cells per sample, and resulted in the exclusion of the following substrates: Atto-550, Atto-565 Atto-620, Atto-633, Atto-647N, Dy-630, Dy-651, and Star-635.

The fluorescent substrates tested exhibited similar levels of nonspecific staining in HeLa cells (data not shown). The nonspecific staining observed did not appear to substantially vary among cells in the same sample, or between the same samples prepared on different days. Dead cells usually showed much more nonspecific staining than healthy cells.

The remaining dye conjugates were incubated with SNAP-tag negative HeLa, MCF7 and H441 cells. Microscopy recordings were taken for each SNAP substrate in the different cell lines, with the focus of the microscope at the basal membrane of the cells. The tracking algorithm provided the number of detected molecules in each frame. For each substrate the camera acquisition time used was the same as that used in the recordings with the SNAP-tag positive cells, see Table S1. This ensured that the number of detectable nonspecific molecules was determined under the same 

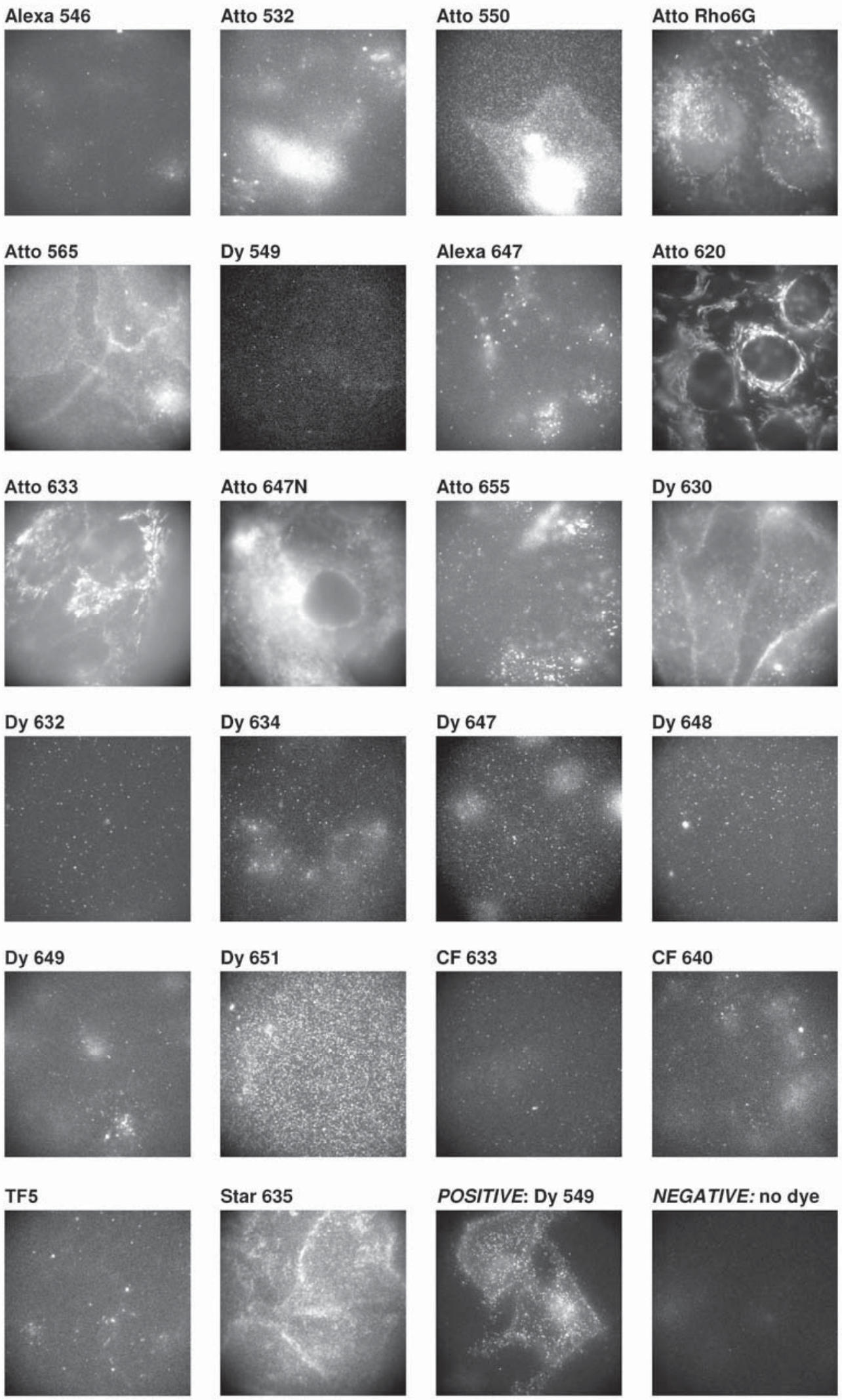

FIGURE 4.2 Fluorescence image of SNAP-tag negative cells incubated with SNAP-tag fluorescent substrates. Incubation of the fluorescent substrates with SNAP-tag negative cells reveals large differences of nonspecific binding to cellular components or the glass surface. Two images showing the staining on SNAPtag positive cells are included for comparison. The images are recorded in widefield mode on a single molecule sensitive microscope. The field of view was completely confluent with cells. The size of the images is $61 \times 61 \mu \mathrm{m}$. The photon intensity scale has not been determined, and varies between images. 
imaging and tracking conditions as for the imaging of specifically bound molecules. Next we differentiated completely immobile molecules from (transiently) mobile molecules. Completely immobile molecules are often molecules bound to the glass substrate; these are typically of less concern, since they can usually be readily excluded before further analysis. In contrast, nonspecific mobile molecules obscure the analysis of the specifically labeled protein molecules.

The average number of mobile molecules as well as the total number of molecules (mobile and immobile) detected in frame numbers $20-40$ are shown in Fig. 4.3. The first 20 frames were excluded because the autofluorescence of the cells is then particularly high, which obscures the specific detection of labeled proteins. Only regions with full confluence of cells were recorded. The total number of nonspecific molecules per field of view is a measure of the expected number of molecules adsorbed on the glass substrate (underneath the cells), the immobile molecules, plus the number of false positive molecules on the plasma membrane, the (transiently) mobile molecules. The number of nonspecific molecules in frame numbers 120-140 is also shown to get insight in the photobleaching of nonspecifically bound substrates.

\subsubsection{Photostability of the substrates bound to SNAP-tag}

To determine the photostability of the dyes bound to SNAP-tag, we incubated them with cells expressing the SNAP-EGFR fusion protein. Microscopy recordings were taken for each dye conjugate to determine the photobleaching rate of the dyes bound to SNAP-tag. A stably transfected HeLa cell line, which had low expression levels of SNAP-EGFR (single molecule density), was utilized to avoid variance between cells of different samples as a result of transfection. We optimized the incubation concentration and time for high labeling efficiency and low nonspecific binding using a titration series with BG-Alexa 546, and found that 2 minutes with $400 \mathrm{nM}$ of substrate were enough for a complete labeling with this dye (and also used these incubation conditions in the nonspecific binding assay). Note that incubation using elevated dye concentrations or prolonged incubation time might result in higher nonspecific binding levels. For each dye conjugate, we observed a similar percentage of labeled cells (estimated to be 15\%) irrespective of the specific dye choice. We believe that this percentage of labeled cells was caused by a large population of cells that do not express the SNAP-tag. The fraction of SNAP-tag receptors labeled in cells appeared to vary slightly from dye to dye.

For an accurate comparison, we aimed to obtain the same number of detected photons per frame (single molecule brightness $B$ ) for all green and all red dyes. All the dyes were bright enough to be detected at a single molecule level in a widefield setup in the presence of cellular autofluorescence background. A widefield setup is more appropriate than a TIRF setup for an accurate comparison as the single molecule brightness B is very difficult to control in TIRF due to varying TIRF angles and the presence of molecules at different depths. Furthermore, the expected number of photons emitted from a fluorophore does not depend on the type of illumination. For the characterization procedure followed, we found that an optimal single molecule brightness was $B=150$ photons for red excitable dyes and $B=200$ photons for green excitable dyes. Some dyes needed a relatively long acquisition time to obtain the targeted single molecule brightness $B$ (see Table $\mathrm{S} 1$ for the acquisition times used and Fig. S2 for the resulting single molecule brightness $B$ ). Table S1 also lists the relative brightness of each dye conjugate to SNAP-tag. At least four videos of different cells were recorded and analyzed per dye conjugate (Fig. 4.4A and Video 1). The brightness (spot intensity) of the molecules follows a Poisson like distribution, as shown in Fig. $4.4 \mathrm{~B}$. 

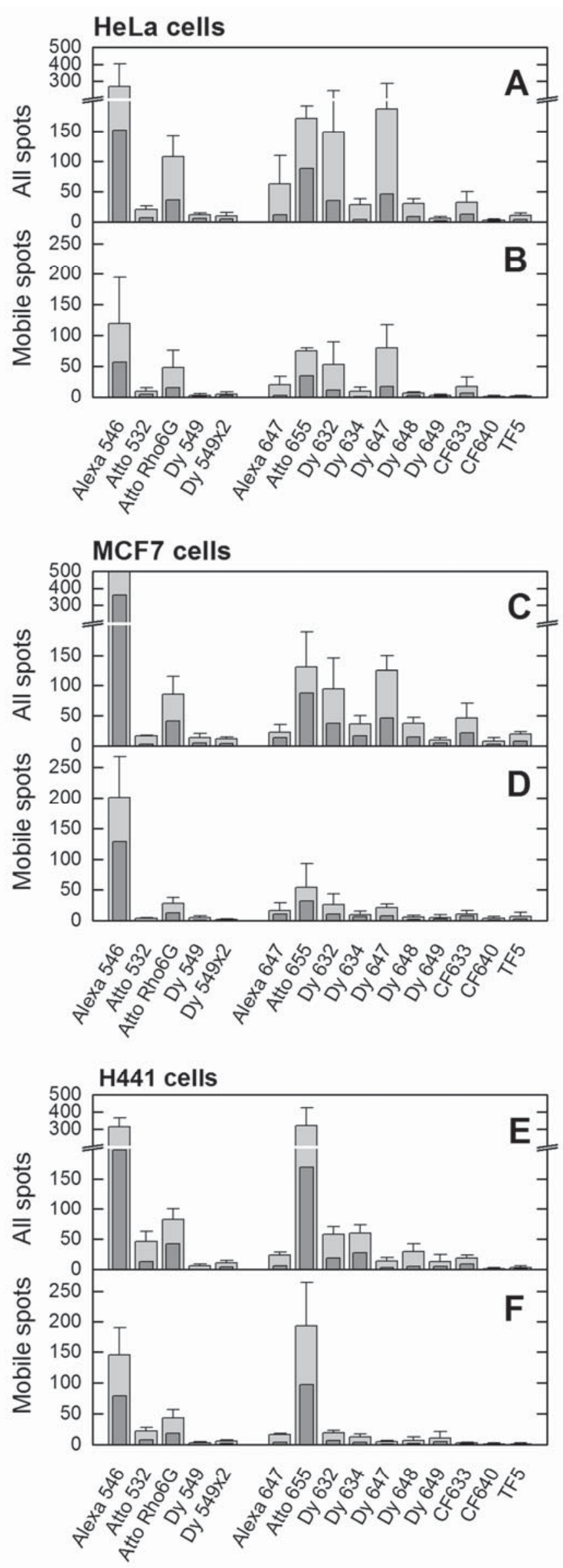

FIGURE 4.3 Quantification of the nonspecific binding of SNAP-tag fluorescent substrates in live cells. The values show the number of mobile and the total number (mobile and immobile) of fluorescent substrates that were nonspecifically bound to cells. The values were determined per field of view area in HeLa, MCF7 and H441 cell lines for each dye showing nonspecific binding on the single molecule level. Some dyes had extremely high levels of nonspecific binding, and since no individual spots could be detected, these were excluded from this graph. Shown are the average number of molecules detected in frames number 20-40 (light grey $\square$ ) and in frames number 120140 (grey $\square$ ). The field of view is a circular area $\left(1520 \mu \mathrm{m}^{2}\right)$ with a radius of approximately $22 \mu \mathrm{m}$. The values were determined in multiple recordings, and the average number is shown here, with the error representing the sample standard deviation. 
Due to photobleaching, the number of observed fluorescent molecules $N(i)$ decreased over time (Fig. 4.4C). We fitted the rate of photobleaching using Eq. 1 to extract the mean photobleaching time $\tau$ for each fluorophore. Using Eq. 2 the expected number of detectable photons per molecule $P_{\text {corr }}$ was calculated. A basal level of detected molecules was observed even after a long imaging time. We believe that these remaining molecules are the result of molecules in an intermittentstate (blinking) and a constant influx of molecules from out-of-focus areas into focus. The expected number of detectable photons per molecule $P_{\text {corr }}$ was obtained from multiple recordings per fluorescent substrate, and the average value and standard deviation are shown in Fig. 4.5. The conversion from numbers of molecules to photons requires that single molecules are detected. This was checked by confirming that the number of emitted photons per molecule does not vary over time (Fig. 4.4D).
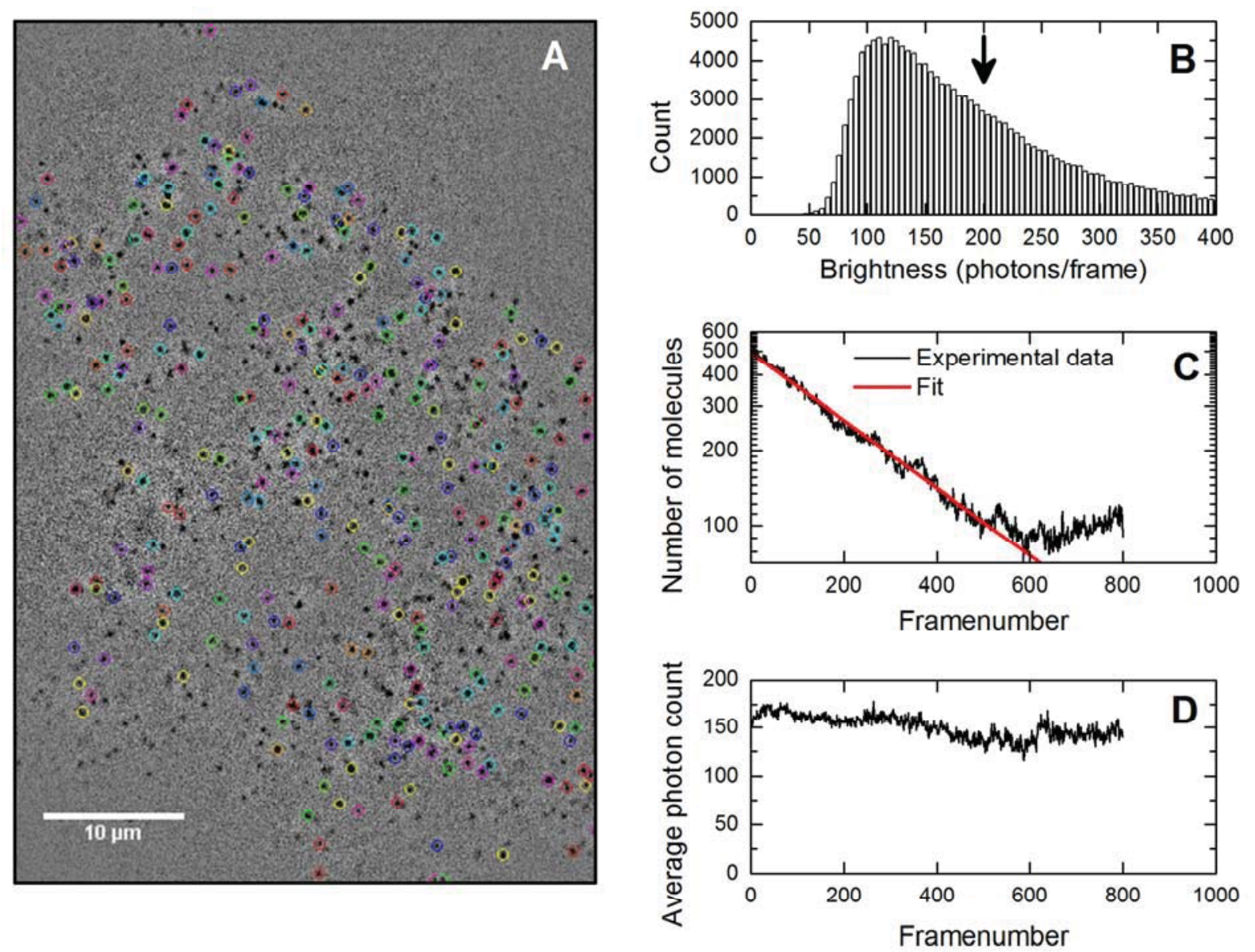

FIGURE 4.4 Example of the performed photobleaching analysis on one video recording.

A fluorescence image series of SNAP-EGFR labeled with a BG-Dy 549 was recorded. (A) The tracking algorithm finds the molecules in the raw microscope recording, and after exclusion of immobile molecules and very short trajectories, the detected molecules are encircled in the microscopy recording where colors are used to differentiate tracks, see also the online Video 1. (B) The histogram of the number of detected photons per frame of all the found molecules (brightness or spot intensity). The arrow indicates the average of the values, which we defined as the single molecule brightness $B$. (C) The number of detected molecules per frame $N(i)$ as function of framenumber $i$. In red, a fit of the data using a single exponent exponential decay function according to Eq. 1 to yield the mean photobleaching time $\tau$ for each fluorophore. (D) The average brightness of the molecules in one frame does not change over time, evidencing that we indeed looked at single molecules. 
In Fig. 4.6 we summarize the results for nonspecific binding versus the photostability for each dye. From this figure it is clear that both green (e.g. Dy 549) and red excitable dyes (e.g. CF633 and CF640) are suitable for single molecule tracking. This result allowed us to examine the possibility to simultaneously label the SNAP-tag with two spectrally different dyes. The simultaneous incubation of a 1.0:0.67 mixture of BG-CF633 and BG-Dy 549 resulted in roughly equal labeling of the SNAP-tag receptor with these two dyes (Fig. 4.7). Video 2 shows SNAP-EGFR proteins labeled with these two dyes diffusing in the plasma membrane of a live cell. The disappearing of receptors is due to photobleaching.
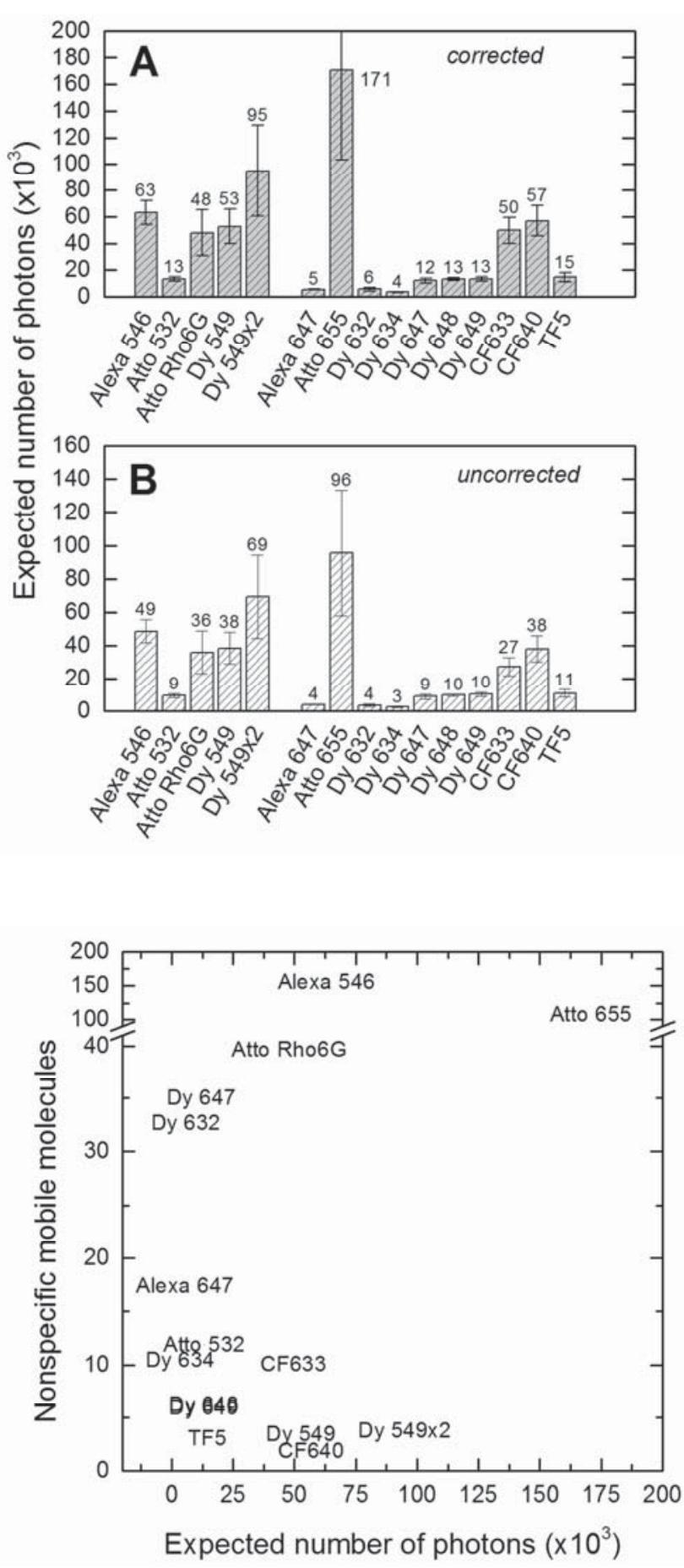

FIGURE 4.5 The expected number of detectable photons per molecule $P_{\text {corr }}$ for each SNAP-tag fluorescent substrate. The expected number of photons provides a value for the photostability of a dye conjugate. The values were determined in multiple recordings, and the average number is shown here, with the error representing the sample standard deviation. (A) The values are corrected for the detection efficiency of the microscope for each dye. (B) The values are not corrected for the detection efficiency, and represent the expected number of photons detected in our setup.

FIGURE 4.6 Comparison of the performance of the SNAP-tag fluorescent substrates for use in single molecule tracking microscopy. The performance is shown in terms of photostability and nonspecific binding. BG-Dy 648 and BG-Dy 649 overlap in the graph. Fluorescent substrates in the lower right corner show little nonspecific attachment to cells, and the most emitted photons per molecule before photobleaching. These substrates are the preferred choice for single molecule tracking microscopy. 
FIGURE 4.7 A TIRF image demonstrating dual color labeling of SNAP-tag receptors at the single molecule level. The labeling was performed on SNAP-EGFR with BGDy549 (green) and BG-CF633 (red). The combination of relatively photostable dye conjugates with little nonspecific staining allows multi-color single molecule tracking microscopy. Using this technique receptor homodimers can be directly visualized. See also Video 2.

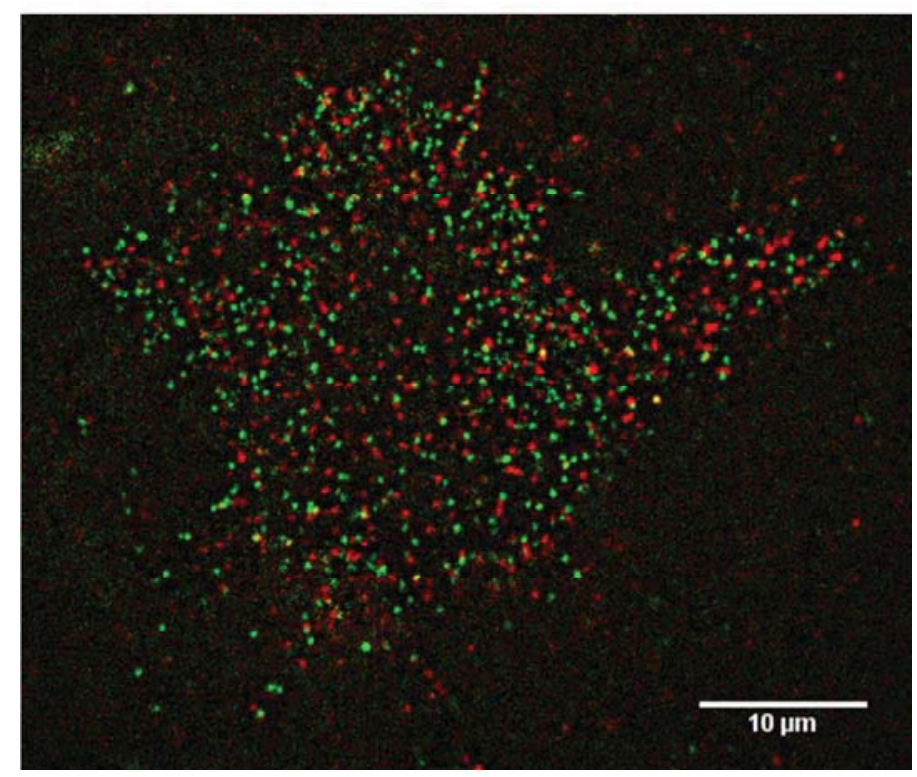

\subsection{Discussion}

The results show that a careful choice of the dye to conjugate to the SNAP-substrate to label SNAP-tag fusion proteins is very important, as many fluorescent substrates either suffer from rapid photobleaching or high nonspecific staining. We found that three fluorescent substrates out of the 22 substrates tested can be used for single molecule tracking applications as these substrates combine both a low level of nonspecific binding and a high photostability. Among the greenexcitable fluorescent substrates, BG-Dy 549 showed the highest photostability with the lowest nonspecific staining (Fig. 4.6). As an alternative, BG-Alexa 546 could be used in ensemble measurements (e.g. FRET studies), as it is photostable and only results in detectable nonspecific binding at the single molecule level. Among the red-excitable fluorescent substrates, BG-CF640 and BG-CF633 exhibited the best results (Fig. 4.6). Whereas BG-CF640 showed slightly lower nonspecific staining, CF633 might be relatively brighter depending on the filter sets available. Even though BG-Atto 655 showed the highest photostability among the substrates tested (Fig. 4.5), its use is limited to ensemble measurements, since it showed high levels of nonspecific binding to membrane components of all the three cell lines (Fig. 4.3).

\subsubsection{Nonspecific binding of the SNAP substrates}

One of the main advantages of single molecule tracking techniques is the ability to discriminate single mobile molecules from cellular autofluorescence, immobile fluorescent molecules, and clusters of fluorescent molecules. We utilized this to characterize the nonspecific binding of the fluorescent substrates. Dealing with the nonspecific binding of fluorophores to any cellular components is one of the biggest challenges in microscopy. Several of the BG-dyes tested showed high levels of nonspecific binding (Fig. 4.2). We found that the amount of nonspecific binding of the BG-dyes is roughly the same among the cell lines tested (Fig. 4.3). None of the dyes that led to appreciable levels of nonspecific staining photobleached within a short period (Fig. 4.3); hence differences in photobleaching of specifically and nonspecifically bound dyes cannot be used advantageously to discriminate between the two cases.

The cause of nonspecific binding might be explained from a molecular perspective. Several dyes contain long chain hydrocarbons which are lipophilic; therefore they easily incorporate in lipid rich structures such as cellular membranes. Sulfonate acid groups are often added to dyes to enhance their solubility in water. These groups are negatively charged and electrostatically 
repelled away from the negatively charged lipid headgroups in cellular membranes. Negatively charged dyes include sulfonated fluorescein- and cyanine-based dyes ${ }^{69}$. On the other hand, cationic (positively charged) dyes, such as many rhodamine based dyes have been reported to bind to mitochondria in live cells ${ }^{70}$. Therefore, the major factors influencing nonspecific binding might be the lipophilic character of a dye in combination with localized electronic charges. Furthermore, the inability of certain dyes to penetrate the plasma membrane hinders access to intracellular structures. In general, neutral and anionic (negatively charged) dyes in this survey appeared to have less tendency to bind to cellular substructures (e.g., Alexa 546/647, Dy 632/634, Dy 648/649). Some dyes (e.g., Atto 647N, Dy 651) adhered in a large extent to the glass coverslip (which may perhaps be avoided by optimizing cleaning procedures), obscuring the detection of specifically bound single molecules in the adjacent plasma membrane of the cell. The complex effects of local charges in combination with polar and lipophilic groups in a dye molecule make it difficult to predict the nonspecific binding ability of dyes beforehand. For example, the net charge of a molecule does not completely explain the nonspecific interaction, such as for the negatively charged BG-Dy 651 and the neutrally charged BG-Dy 630. Both showed a considerable amount of nonspecific binding to cellular components. We also did not find a correlation between the chromophore family and the nonspecific labeling level. For example, the incubation of cells with the rhodamine derived dyes BG-Alexa 546 and BG-Atto 532 resulted in low nonspecific levels, while BG-Atto 550 and BG-Atto 565 led to much higher nonspecific levels. Likewise the cyanine based BG-Alexa 647 showed almost no nonspecific binding, while the BG-Dy 630 exhibited extremely high nonspecific binding.

Benke et al. have reported the use of five BG dyes for single molecule tracking in eukaryotic cells $^{61}$. In their approach, the fluorescence of these dyes was stochastically activated for superresolution microscopy; however, no data on nonspecific binding was provided. Stöhr et al. described the quenching of several dyes after conjugation to BG and subsequent SNAP-tag binding ${ }^{63,71}$. Their data demonstrates that the photophysics (i.e. the photobleaching time and fluorescence quenching) of a given dye can be altered by its molecular environment. Furthermore they conclude that it is impossible to predict the changes in fluorescence beforehand due to the complex effects of local charges in the dye molecule. Stöhr et al. also reported on the background levels of remaining unreacted dyes inside E. coli after washing protocols. Interestingly, some substrates such as BG-Atto 620 and BG-Atto 633, which reportedly exhibited a low background staining in $E$. coli, led to a surprisingly high nonspecific binding in our experiments with mammalian cell lines. Stöhr et al. also reported the labeling of 3T3 fibroblast cells with BG-Atto 550, BG-Atto 633 and BG-Atto 647N. Similarly, we noticed that BG-Atto 550 and BG-Atto 647N produced high levels of nonspecific binding. However, in contrast to Stöhr et al., in our hands BGAtto 633 showed a very intense nonspecific staining of cytosolic and membrane structures (Fig. 4.2 and Fig. S2). This discrepancy could be caused by the different fluorescence intensity levels in both studies, as we looked at nonspecific staining in the context of single molecules.

\subsubsection{Photostability of the substrates bound to SNAP-tag}

Whereas many red-excitable dye conjugates did not show any substantial nonspecific binding levels, these dyes appeared to be less photostable than the green-excitable dyes. Two dyes (CF633 and CF640) are photostable enough to permit prolonged imaging with low nonspecific staining (Fig. 4.6). Between these two dyes, CF640 showed marginally less nonspecific staining (Fig. 4.3).

Another noteworthy observation was that the photostabilities of the Dy dyes of relatively close excitation wavelengths were very similar (Fig. 4.5), for instance Dy 647, 648 and 649, and also Dy 632 and 634. From a molecular perspective, Dy 647, 648, and 649 are typical cyanine dyes, while Dy 632 and 634 have one indole group with a polymethine chain linked to a benzopyrylium 
group. The slight differences in these chromophores did not seem to have a large effect on its photobleaching rate.

\subsubsection{Complications and validity}

We have performed the photobleaching experiments on SNAP-tag fused transmembrane EGFR proteins, which have a basal internalization rate even when the cells are starved ${ }^{72,73}$. This might lead to a false enhanced bleaching detection. During the 30 seconds of imaging, however, the internalisation rate of the receptor is small compared to the photobleaching rate ${ }^{74}$. Even one hour after the labeling, no significant decrease of receptor molecules was observed at the plasma membrane of the cells. However, in some instances a small increase in fluorescence in the cytosol was noticeable, which could be an attributed to the basal level of receptor internalization and the recycling process. Another complication stems from the fact that this receptor seems to localize more in filopodia and the periphery of the cells $\mathrm{s}^{75}$; these receptors can diffuse more easily in and out of focus. Because molecules diffusing in and out of the plane of focus will likely be in equilibrium, this should not influence the recorded bleaching rate at the beginning of a recording when receptors in focus have not been bleached yet. Later, however, while bleached receptors leave the plane, unbleached receptors can enter the focal plane from outside the plane, causing a slower bleaching rate than the actual rate. Therefore, we derived the bleaching rate from that part of the recordings where the number of molecules is still decreasing. Furthermore, the rate of receptors entering the focal plane within the 30 seconds of imaging will be limited, and this rate will be independent of the dye used.

\subsubsection{Improvements to fluorescent SNAP substrates}

The attachment of two Dy 549 dyes on a single SNAP substrate (Dy 549x2) seems an interesting approach to prolong imaging of the protein, as its photostability almost doubled compared to the substrate with single loaded Dy 549 (Fig. 4.5). The brightness of the double loaded SNAP substrate was similar to the single loaded substrate (Table S1). This might be due to self-quenching, which is commonly observed when increasing the number of fluorophores on a protein, and affects the fluorescence intensity but not the photobleaching rate per photon for the complex. Further studies are needed to confirm that the (single molecule) brightness is indeed similar in SNAP substrates with one, two, or even more Dy 549 fluorophores.

Another interesting point is the incorporation of a strong fluorescence quencher in the guanine group. Such a fluorogenic method ensures that the benzylguanine coupled fluorophore becomes dramatically more fluorescent upon binding to the SNAP-tag ${ }^{40}$. Although the guanine itself already acts as a relatively good quencher for several dyes $^{63}$, the more dramatic fluorogenic approach could bypass the issue of nonspecific binding for extremely photostable dyes such as Atto-655. Another interesting idea is to use a SNAP-tag substrate derivatized with a fluorophore and a triplet-state quencher (e.g. a molecular oxygen reducing agent $)^{76}$. This strategy has led to an overall decrease of dark state transitions which led to up to 25 fold longer imaging periods ${ }^{76}$. Prolonged imaging might allow to observe and follow many more interactions of the protein on its path through the cell.

\subsection{Conclusions}

We have screened and analyzed the photostability and nonspecific binding properties of a wide range of green- and red-excitable dyes for labeling proteins in cells by means of the SNAP-tag technology. The SNAP-tag labeling strategy is particularly useful for labeling proteins on the plasma membrane, since there are no restrictions on the membrane permeability of the fluorescent label. Properties of dyes have generally been determined in ensemble fluorescence imaging and on relatively large biomolecules such as antibodies. However, properties of dyes can 
be rather different at the single molecule level and when conjugated on a small biomolecule, such as the SNAP substrate (BG), and in the local microenvironment of the SNAP-tag. We have characterized the photostability and specificity for several SNAP-substrate dye conjugates in different cell lines at a single molecule level. We performed the characterization in widefield mode to prevent illumination variations experienced in a TIRF setup, and at high single molecule brightness to adequately count most dye molecules in the recordings. To provide a meaningful comparison, we used similar photon counts per single molecule for each spectrally similar dye, corrected for the detection efficiency of our microscope for the dye's emission spectrum, and performed the tracking of the bound dyes to differentiate the motion of the nonspecifically bound molecules.

We found that in our system the SNAP substrates labeled with Dy 549, CF633, and CF 640 are the best choices to label SNAP-tag fusion proteins for single molecule tracking among the fluorescent substrates tested. Also, we have shown that the attachment of two Dy-549 dyes on one BG probe is an interesting approach to prolong imaging of the protein. Finding two spectrally different dyes for labeling SNAP-tag that were suitable for single molecule imaging proved to be a challenge, as most of the fluorescent substrates tested showed either a large amount of nonspecific fluorescence or were rapidly photobleached.

Since both green- and red-excitable fluorescent SNAP substrates have been identified, multicolor single molecule imaging of the same protein species can become a routine experiment by simply simultaneously incubating these substrates with the SNAP-tag fusion proteins in live cells. This should allow the direct observation of homo-dimers. For an extension to three-color single molecule imaging, BG-Alexa 488 could be used since it is already known to be a suitable dye for single molecule tracking ${ }^{61}$, although the intense cellular autofluorescence at this wavelength limits its use to total internal reflection fluorescence (TIRF) microscopy. We anticipate that our conclusions could be extended to the chemically similar tagging technology CLIP-tag, which also has the guanine moiety in its substrate. Our results are probably not directly translatable to chemically different molecular tags, such as Halo, ACP and MCP. The combination of SNAP-tag with another molecular labeling tag allows orthogonal labeling on two different protein species. An interesting extension to one protein species studies is therefore the direct visualization of two interacting proteins of different protein species, such as in heterodimer formation.

\section{Acknowledgement}

We thank Jenny Ibach for providing plasmid for SNAP-EGFR; we thank Yvonne Kraan for assistance in cell culturing and sample preparations; and we thank Peter Relich and Keith Lidke for sharing their tracking software with us. This work is supported by an EU NanoSci E+ grant via STW grant 11022-NanoActuate. 


\subsection{References}

1. Kusumi, A. et al. Paradigm shift of the plasma membrane concept from the two-dimensional continuum fluid to the partitioned fluid: high-speed single-molecule tracking of membrane molecules. Annu. Rev. Biophys. Biomol. Struct. 34, 351-78 (2005).

2. Xia, T., Li, N. \& Fang, X. Single-molecule fluorescence imaging in living cells. Annu. Rev. Phys. Chem. 64, 459-80 (2013).

3. Joo, C., Balci, H., Ishitsuka, Y., Buranachai, C. \& Ha, T. Advances in single-molecule fluorescence methods for molecular biology. Annu. Rev. Biochem. 77, 51-76 (2008).

4. Hinterdorfer, P. \& van Oijen, A. Handbook of SingleMolecule Biophysics. (Springer Science+Business Media, 2009).

5. Joo, C., Fareh, M. \& Narry Kim, V. Bringing singlemolecule spectroscopy to macromolecular protein complexes. Trends Biochem Sci 38, 30-37 (2013).

6. Lippincott-Schwartz, J., Snapp, E. \& Kenworthy, A. Studying protein dynamics in living cells. Nat. Rev. Mol. Cell Biol. 2, 444-56 (2001).

7. Miyawaki, A. Proteins on the move: insights gained from fluorescent protein technologies. Nat. Rev. Mol. Cell Biol. 12, 656-68 (2011).

8. Giepmans, B. N. G., Adams, S. R., Ellisman, M. H. \& Tsien, R. Y. The fluorescent toolbox for assessing protein location and function. Science 312, 217-24 (2006).

9. Pantoja, R., Rodriguez, E. A., Dibas, M. I., Dougherty, D. A. \& Lester, H. A. Single-Molecule Imaging of a Fluorescent Unnatural Amino Acid Incorporated Into Nicotinic Receptors. Biophys. J. 96, 226-237 (2009).

10. Callegari, A. et al. Single particle tracking of acyl carrier protein (ACP)-tagged TrkA receptors in PC12nnr5 cells. J. Neurosci. Methods 204, 82-86 (2012).

11. Low-Nam, S. T. et al. ErbB1 dimerization is promoted by domain co-confinement and stabilized by ligand binding. Nat. Struct. Mol. Biol. 18, 1244-1249 (2011).

12. Goulian, M. \& Simon, S. M. Tracking single proteins within cells. Biophys. J. 79, 2188-98 (2000).

13. Sako, Y., Minoghchi, S. \& Yanagida, T. Singlemolecule imaging of EGFR signalling on the surface of living cells. Nat. Cell Biol. 2, 168-72 (2000).

14. Wieser, S. \& Schütz, G. J. Tracking single molecules in the live cell plasma membrane-Do's and Don't's. Methods 46, 131-40 (2008).

15. Sergé, A., Bertaux, N., Rigneault, H. \& Marguet, D. Dynamic multiple-target tracing to probe spatiotemporal cartography of cell membranes. Nat. Methods 5, 687-94 (2008).

16. Kholodenko, B. N., Hancock, J. F. \& Kolch, W. Signalling ballet in space and time. Nat. Rev. Mol. Cell Biol. 11, 414-426 (2010).

17. Cambi, A. \& Lidke, D. S. Nanoscale membrane organization: where biochemistry meets advanced microscopy. ACS Chem. Biol. 7, 139-49 (2012).

18. Saxton, M. J. \& Jacobson, K. Single-particle tracking: applications to membrane dynamics. Annu. Rev. Biophys. Biomol. Struct. 26, 373-99 (1997).

19. Kapanidis, A. N. \& Strick, T. Biology, one molecule at a time. Trends Biochem. Sci. 34, 234-43 (2009).
20. Schütz, G. J., Schindler, H. \& Schmidt, T. Singlemolecule microscopy on model membranes reveals anomalous diffusion. Biophys. J. 73, 1073-80 (1997).

21. Brameshuber, M. \& Schütz, G. J. in Springer Series on Fluorescence 1-37 (Springer Berlin Heidelberg, 2012). doi:10.1007/4243_2011_38

22. Dietrich, C., Yang, B., Fujiwara, T., Kusumi, A. \& Jacobson, K. Relationship of lipid rafts to transient confinement zones detected by single particle tracking. Biophys. J. 82, 274-84 (2002).

23. De Keijzer, S. et al. A spatially restricted increase in receptor mobility is involved in directional sensing during Dictyostelium discoideum chemotaxis. J. Cell Sci. 121, 1750-7 (2008).

24. Cutler, P. J. et al. Multi-color quantum dot tracking using a high-speed hyperspectral line-scanning microscope. PLoS One 8, e64320 (2013).

25. Cebecauer, M., Spitaler, M., Sergé, A. \& Magee, A. I. Signalling complexes and clusters: functional advantages and methodological hurdles. J. Cell Sci. 123, 309-20 (2010).

26. Harding, A. S. \& Hancock, J. F. Using plasma membrane nanoclusters to build better signaling circuits. Trends Cell Biol. 18, 364-371 (2008).

27. Radhakrishnan, K., Halász, Á., McCabe, M. M., Edwards, J. S. \& Wilson, B. S. Mathematical simulation of membrane protein clustering for efficient signal transduction. Ann. Biomed. Eng. 40, 2307-18 (2012).

28. Dunne, P. D. et al. DySCo: quantitating associations of membrane proteins using two-color singlemolecule tracking. Biophys. J. 97, L5-7 (2009).

29. Filonov, G. S. et al. Bright and stable near-infrared fluorescent protein for in vivo imaging. Nat. Biotechnol. 29, 757-61 (2011).

30. Harms, G. S., Cognet, L., Lommerse, P. H., Blab, G A. \& Schmidt, T. Autofluorescent proteins in singlemolecule research: applications to live cell imaging microscopy. Biophys. J. 80, 2396-408 (2001).

31. Wieser, S., Axmann, M. \& Schütz, G. J. Versatile analysis of single-molecule tracking data by comprehensive testing against Monte Carlo simulations. Biophys. J. 95, 5988-6001 (2008).

32. Howarth, M. et al. Monovalent, reduced-size quantum dots for imaging receptors on living cells. Nat. Methods 5, 397-9 (2008).

33. Liu, H. Y. \& Gao, X. Engineering monovalent quantum dot-antibody bioconjugates with a hybrid gel system. Bioconjug. Chem. 22, 510-7 (2011).

34. Farlow, J. et al. Formation of targeted monovalent quantum dots by steric exclusion. Nat. Methods 10, 1203-5 (2013).

35. Pinaud, F. F. et al. Quantum dots for live cells, in vivo imaging, and diagnostics. Science 307, 538-44 (2005).

36. Pinaud, F., Clarke, S., Sittner, A. \& Dahan, M. Probing cellular events, one quantum dot at a time. Nat. Methods 7, 275-85 (2010).

37. Keppler, A. et al. A general method for the covalent labeling of fusion proteins with small molecules in vivo. Nat. Biotechnol. 21, 86-9 (2003).

38. Keppler, A., Pick, H., Arrivoli, C., Vogel, H. \& Johnsson, K. Labeling of fusion proteins with synthetic fluorophores in live cells. Proc. Natl. Acad. Sci. U. S. A. 101, 9955-9 (2004). 
39. Gautier, A. et al. An engineered protein tag for multiprotein labeling in living cells. Chem. Biol. 15, 128-36 (2008)

40. Sun, X. et al. Development of SNAP-tag fluorogenic probes for wash-free fluorescence imaging. Chembiochem 12, 2217-26 (2011).

41. Corrêa, I. R. et al. Substrates for improved live-cell fluorescence labeling of SNAP-tag. Curr. Pharm. Des. 19, 5414-20 (2013).

42. Pellett, P. A. et al. Two-color STED microscopy in living cells. Biomed Opt Express 2, 2364-2371 (2011).

43. Dempsey, G. T., Vaughan, J. C., Chen, K. H., Bates M. \& Zhuang, X. Evaluation of fluorophores for optimal performance in localization-based superresolution imaging. Nat. Methods 8, 1027-36 (2011).

44. Klein, T. et al. Live-cell dSTORM with SNAP-tag fusion proteins. Nat. Methods 8, 7-9 (2011).

45. Foraker, A. B. et al. Clathrin promotes centrosome integrity in early mitosis through stabilization of centrosomal ch-TOG. J. Cell Biol. 198, 591-605 (2012).

46. Bojkowska, K. et al. Measuring In Vivo Protein HalfLife. Chem. Biol. 18, 805-815 (2011).

47. Ville, D. et al. Ring 14 chromosome presenting as early-onset isolated partial epilepsy. Dev. Med. Child Neurol. 51, 917-22 (2009).

48. Masharina, A., Reymond, L., Maurel, D., Umezawa K. \& Johnsson, K. A fluorescent sensor for GABA and synthetic GABA(B) receptor ligands. J Am Chem Soc 134, 19026-19034 (2012)

49. Chidley, C., Haruki, H., Pedersen, M. G., Muller, E. \& Johnsson, K. A yeast-based screen reveals that sulfasalazine inhibits tetrahydrobiopterin biosynthesis. Nat. Chem. Biol. 7, 375-83 (2011)

50. Hoskins, A. A. et al. Ordered and dynamic assembly of single spliceosomes. Science 331, 1289-95 (2011).

51. Breitsprecher, D. et al. Rocket launcher mechanism of collaborative actin assembly defined by singlemolecule imaging. Science 336, 1164-8 (2012).

52. Jaiswal, R. et al. The formin Daam 1 and fascin directly collaborate to promote filopodia formation. Curr Biol 23, 1373-1379 (2013).

53. Smith, B. A., Daugherty-Clarke, K., Goode, B. L. \& Gelles, J. Pathway of actin filament branch formation by Arp2/3 complex revealed by single-molecule imaging. Proc Natl Acad Sci U S A 110, 1285-1290 (2013)

54. Smith, B. A. et al. Three-color single molecule imaging shows WASP detachment from Arp2/3 complex triggers actin filament branch formation. Elife 2, e01008 (2013).

55. Peisley, A. et al. Kinetic mechanism for viral dsRNA length discrimination by MDA5 filaments. Proc. Natl. Acad. Sci. U. S. A. 109, E3340-9 (2012).

56. Qiu, W. et al. Dynein achieves processive motion using both stochastic and coordinated stepping. Nat. Struct. Mol. Biol. 19, 193-200 (2012).

57. Derr, N. D. et al. Tug-of-war in motor protein ensembles revealed with a programmable DNA origami scaffold. Science 338, 662-5 (2012).

58. Numata, N., Shima, T., Ohkura, R., Kon, T. \& Sutoh, K. C-sequence of the Dictyostelium cytoplasmic dynein participates in processivity modulation. FEBS Lett 585, 1185-1190 (2011).

59. Yang, Y. \& Zhang, C. Y. Simultaneous Measurement of SUMOylation using SNAP/CLIP-Tag-Mediated Translation at the Single-Molecule Level. Angew Chem Int Ed Engl 52, 691-694 (2013).
60. Calebiro, D. et al. Single-molecule analysis of fluorescently labeled G-protein-coupled receptors reveals complexes with distinct dynamics and organization. Proc Natl Acad Sci U S A 110, 743-748 (2013).

61. Benke, A., Olivier, N., Gunzenhäuser, J. \& Manley, $\mathrm{S}$. Multicolor single molecule tracking of stochastically active synthetic dyes. Nano Lett. 12, 2619-24 (2012).

62. Schenk, A., Ivanchenko, S., Röcker, C., Wiedenmann, J. \& Nienhaus, G. U. Photodynamics of red fluorescent proteins studied by fluorescence correlation spectroscopy. Biophys. J. 86, 384-94 (2004).

63. Stöhr, K. et al. Quenched substrates for live-cell labeling of SNAP-tagged fusion proteins with improved fluorescent background. Anal. Chem. 82, 8186-93 (2010).

64. Wang, T. Y. et al. The covalent trimethoprim chemical tag facilitates single molecule imaging with organic fluorophores. Biophys. J. 106, 272-8 (2014).

65. Lukinavicius, G. et al. A near-infrared fluorophore for live-cell super-resolution microscopy of cellular proteins. Nat Chem 5, 132-139 (2013).

66. Smith, B. A. et al. Three-color single molecule imaging shows WASP detachment from Arp2/3 complex triggers actin filament branch formation. Elife 2, e01008 (2013)

67. Smith, C. S., Joseph, N., Rieger, B. \& Lidke, K. A. Fast, single-molecule localization that achieves theoretically minimum uncertainty. Nat. Methods 7 373-5 (2010)

68. Elliott, L. C. C., Barhoum, M., Harris, J. M. \& Bohn, P. W. Trajectory analysis of single molecules exhibiting non-brownian motion. Phys. Chem. Chem. Phys. 13, 4326-34 (2011).

69. Panchuk-Voloshina, N. et al. Alexa Dyes, a Series of New Fluorescent Dyes that Yield Exceptionally Bright, Photostable Conjugates. J. Histochem. Cytochem. 47, 1179-1188 (1999).

70. Johnson, L. V, Walsh, M. L., Bockus, B. J. \& Chen, $\mathrm{L}$. B. Monitoring of relative mitochondrial membrane potential in living cells by fluorescence microscopy. $J$. Cell Biol. 88, 526-35 (1981).

71. Stöhr, K. Fluoreszenzgelöschte Sonder für dianostische und analytische Anwendungen, PhD thesis, Heidelberg University. (2008).

72. Citri, A. \& Yarden, Y. EGF-ERBB signalling: towards the systems level. Nat. Rev. Mol. Cell Biol. 7, 505-16 (2006).

73. Wang, Q., Villeneuve, G. \& Wang, Z. Control of epidermal growth factor receptor endocytosis by receptor dimerization, rather than receptor kinase activation. EMBO Rep. 6, 942-8 (2005).

74. Hofman, E. G. et al. Ligand-induced epidermal growth factor receptor (EGFR) oligomerization is kinase-dependent and enhances internalization. J. Biol. Chem. 285, 39481-39489 (2010).

75. Lidke, D. S. et al. Quantum dot ligands provide new insights into erbB/HER receptor-mediated signal transduction. Nat. Biotechnol. 22, 198-203 (2004).

76. Altman, R. B. et al. Enhanced photostability of cyanine fluorophores across the visible spectrum. Nat. Methods 9, 428-9 (2012). 


\section{Supporting Information}

\section{Evaluation of fluorophores to label SNAP-tag fused proteins for multicolor single molecule tracking microscopy in live cells}

Peter J. Bosch ${ }^{1}$

Ivan R. Corrêa Jr. ${ }^{2}$ Michael H. Sonntag ${ }^{3}$

Luc Brunsveld ${ }^{3}$ Johannes S. Kanger ${ }^{1}$ Vinod Subramaniam $^{1,4}$

1. Nanobiophysics, MESA+ Institute for Nanotechnology and MIRA Institute for Biomedical Technology and Technical Medicine, University of Twente, PO Box 217, 7500AE Enschede, The Netherlands

2. New England Biolabs, Ipswich, MA, USA

3. Laboratory of Chemical Biology, Department of Biomedical Engineering, and Institute of Complex Molecular Systems, Eindhoven University of Technology, The Netherlands

4. Present address: FOM Institute AMOLF, Science Park 104, 1098 XG Amsterdam, The Netherlands 


\section{Supporting figures}

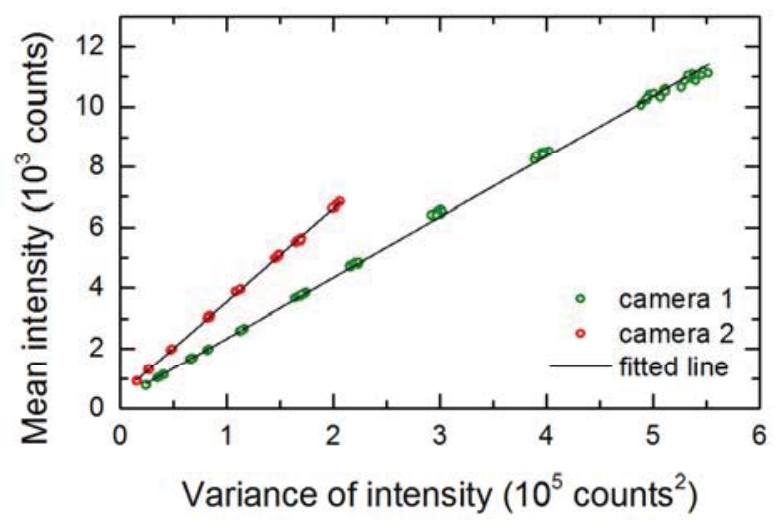

FIGURE S1 Calibration of the gain of the two EMCCD cameras performed by the mean-variance method. The slope of the line in this curve is equal to the inverse gain of the camera. A gain of $49.9 \pm 0.1$ was found for the camera recording the green-excitable dyes, and a gain of $32.5 \pm 0.1$ was found for the camera recording the red-excitable dyes.

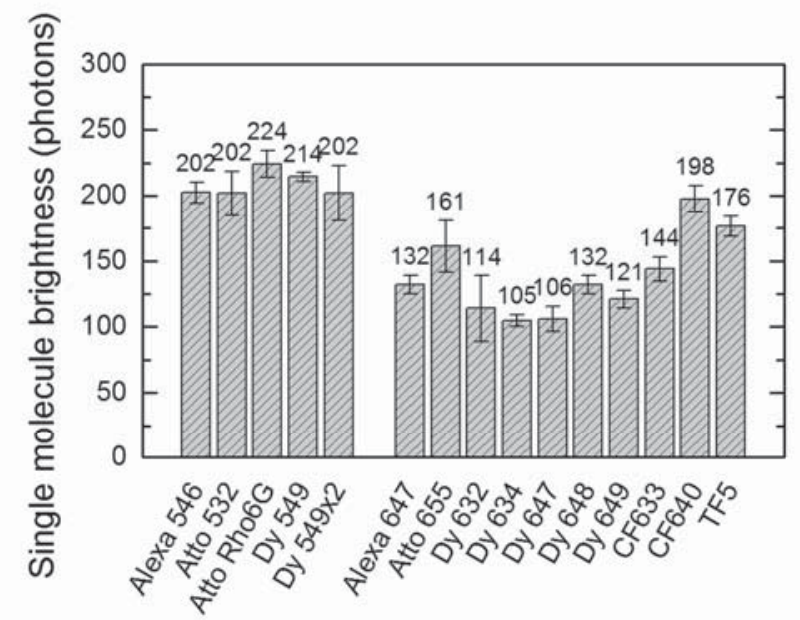

FIGURE S2 The single molecule brightness $B$ per candidate dye.

Some dyes had extremely high nonspecific level, and since no individual spots could be detected, these are excluded from this graph. Note that the single molecule brightness B was determined without corrections for setup dependent parameters, and therefore differs from the relative intensity of a dye (given in Table S1). The error bars represent the sample standard deviation among multiple videos of the same dye. 
FIGURE S3 (below) Fluorescence images of nonspecific binding of the SNAP substrates. Images were recorded in widefield and TIRF illumination setup. The negative images are recordings of SNAP-tag negative H441 cells. The positive images are recordings of SNAP-tag positive HeLa cells. The size of the images is $61 \times 61 \mathrm{um}$, the photon intensity scale has not been determined, and varies between the shown images. Some images were not acquired (N/A) since it was already clear from nonspecific images that a positive image would not be meaningful.

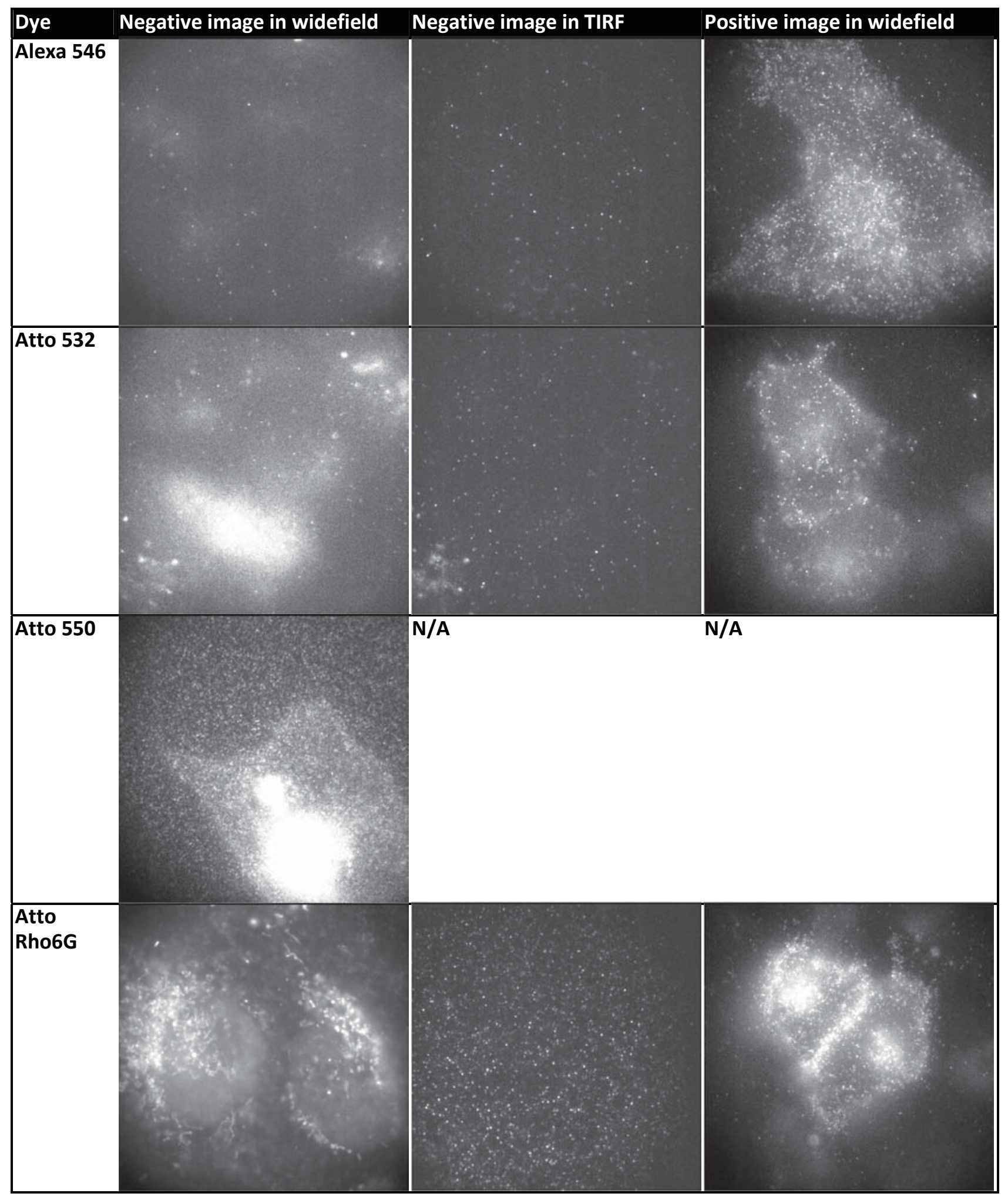




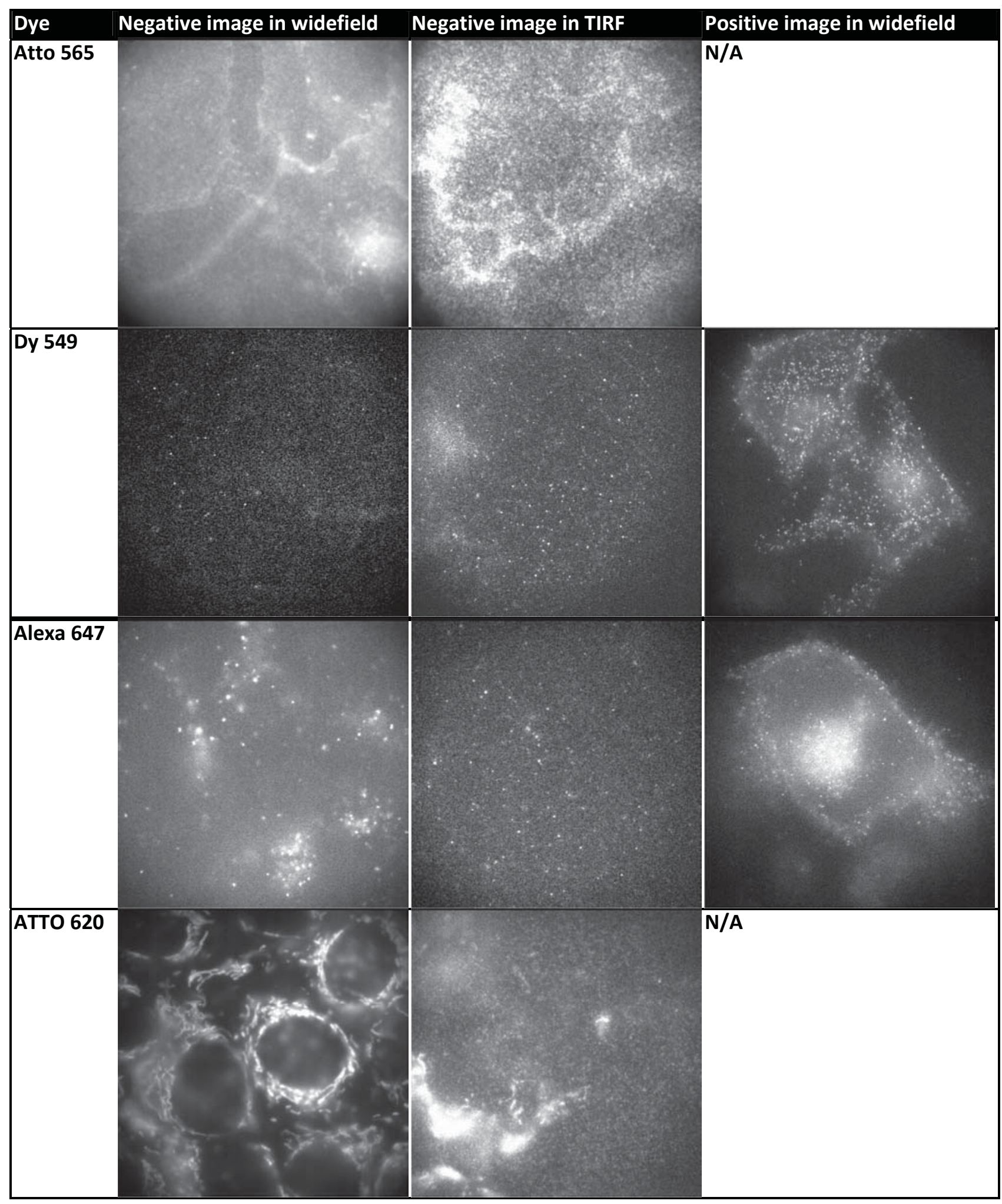


Chapter 4






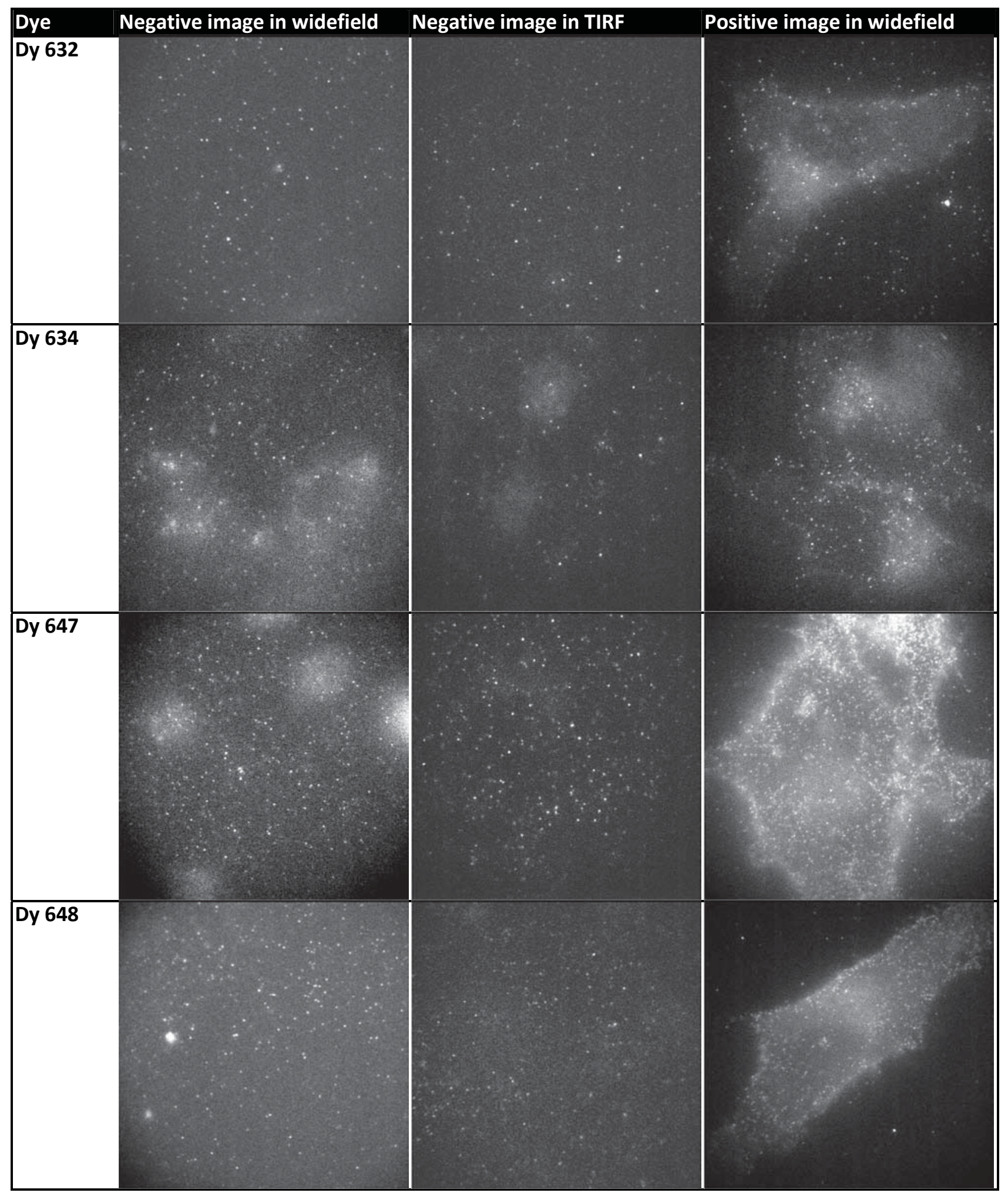




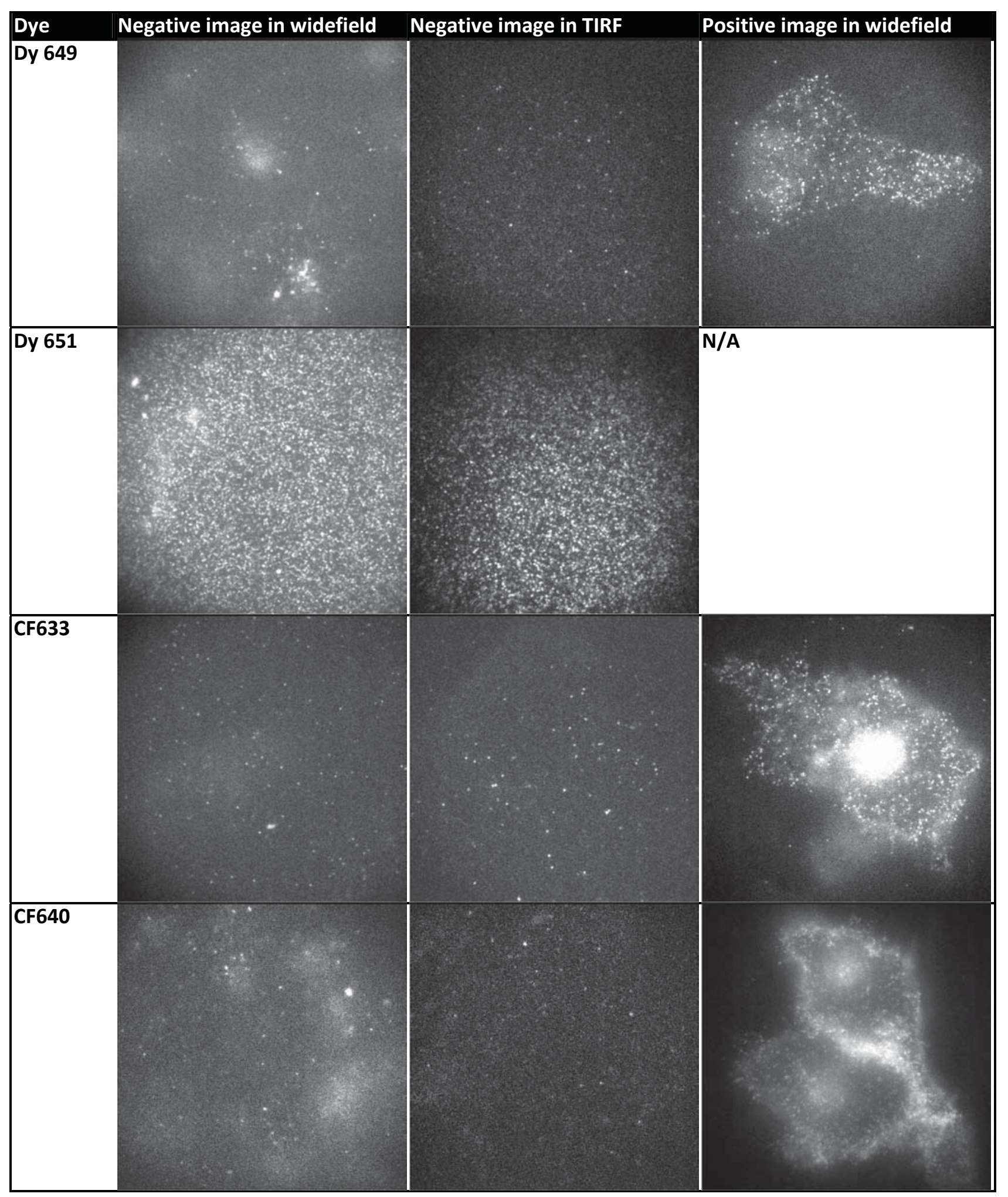




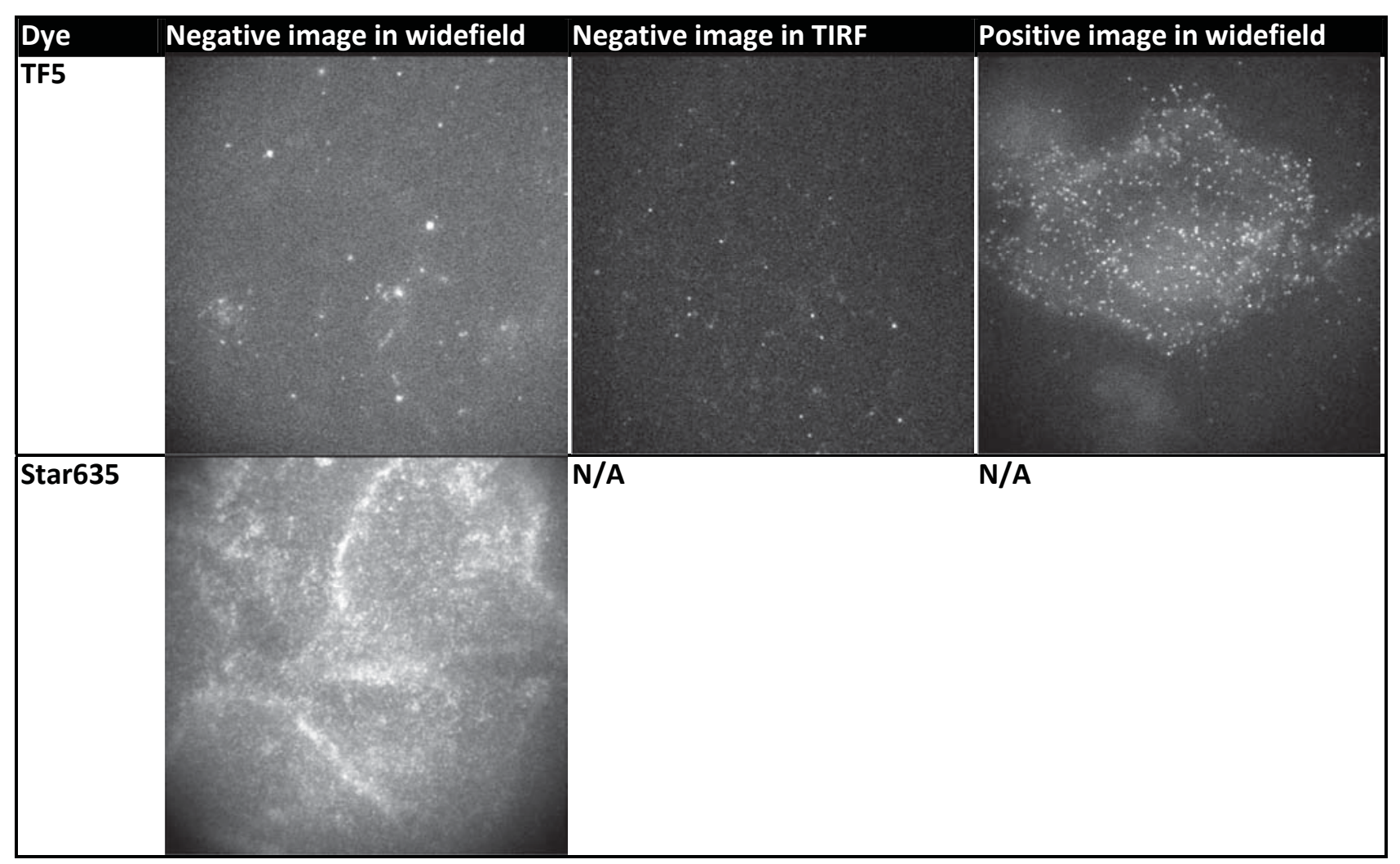




\section{Supporting tables}

Table S1 Efficiency of the excitation and detection of fluorescence in our setup, acquisition time used for microscopy recordings, and resulting relative brightness of the SNAP-tag fluorescent substrates.

\begin{tabular}{|lllll|}
\hline BG-dye & $\begin{array}{l}\text { Excitation } \\
\text { efficiency }\end{array}$ & $\begin{array}{l}\text { Detection } \\
\text { efficiency }\end{array}$ & $\begin{array}{l}\text { Acquisition } \\
\text { Time (ms) }\end{array}$ & $\begin{array}{l}\text { Relative } \\
\text { brightness (\%) }\end{array}$ \\
\hline Alexa 546 & 0.32 & 0.77 & 15 & 43 \\
\hline Atto 532 & 1.00 & 0.71 & 10 & 22 \\
\hline Atto 550 & 0.40 & 0.72 & $\mathrm{n} / \mathrm{a}^{1}$ & $\mathrm{n} / \mathrm{a}$ \\
\hline Atto Rho6G & 0.98 & 0.75 & 15 & 16 \\
\hline Dy 549 & 0.55 & 0.73 & 15 & 28 \\
\hline Dy 549x2 & 0.55 & 0.73 & 15 & 26 \\
\hline Alexa 647 & 0.62 & 0.76 & 7 & 58 \\
\hline ATTO 620 & 0.38 & 0.46 & $\mathrm{n} / \mathrm{a}$ & $\mathrm{n} / \mathrm{a}$ \\
\hline Atto 633 & 0.87 & 0.56 & $\mathrm{n} / \mathrm{a}$ & $\mathrm{n} / \mathrm{a}$ \\
\hline Atto 647N & 0.83 & 0.63 & $\mathrm{n} / \mathrm{a}$ & $\mathrm{n} / \mathrm{a}$ \\
\hline Atto 655 & 0.62 & 0.56 & 19 & 36 \\
\hline Dy 630 & 1.00 & 0.60 & $\mathrm{n} / \mathrm{a}$ & $\mathrm{n} / \mathrm{a}$ \\
\hline Dy 632 & 1.00 & 0.63 & 3 & 88 \\
\hline Dy 634 & 0.83 & 0.73 & 3 & 84 \\
\hline Dy 647 & 0.65 & 0.74 & 4 & 80 \\
\hline Dy 648 & 0.65 & 0.74 & 4 & 100 \\
\hline Dy 649 & 0.66 & 0.74 & 4 & 86 \\
\hline Dy 651 & 0.64 & 0.75 & $\mathrm{n} / \mathrm{a}$ & $\mathrm{n} / \mathrm{a}$ \\
\hline CF633 & 1.00 & 0.75 & 8 & 48 \\
\hline CF640 & 0.85 & 0.67 & 8 & 63 \\
\hline TF5 & 0.78 & 0.74 & 7 & 64 \\
\hline Star635 & 0.99 & 0.57 & $\mathrm{n} / \mathrm{a}$ & $\mathrm{n} / \mathrm{a}$ \\
\hline
\end{tabular}

${ }^{1}$ not acquired, as these substrates showed nonspecific staining above the single molecule level. 
SNAP-tag in single-molecule microscopy

Table S2 Characteristics of the fluorescent substrates in SNAP-tag negative cell lines

Numbers are rounded to the nearest integer.

\section{Negative control H441 cells}

\begin{tabular}{|c|c|c|c|c|c|c|c|c|}
\hline \multirow{2}{*}{$\begin{array}{l}\text { Dye } \\
\text { Alexa } 546\end{array}$} & \multirow{2}{*}{$\begin{array}{l}\text { Raw ph/spot } \\
184\end{array}$} & \multicolumn{3}{|c|}{$\begin{array}{l}\text { Total spots } \\
\text { frame } 20-40\end{array}$} & \multicolumn{3}{|c|}{$\begin{array}{l}\text { Total spots } \\
\text { frame } 120-140\end{array}$} & \multirow{2}{*}{$\begin{array}{l}\text { Mobile spots fraction } \\
\text { frame } 20-40 \\
0.46\end{array}$} \\
\hline & & 315 & \pm & 50 & 198 & \pm & 32 & \\
\hline Atto 532 & 176 & 47 & \pm & 17 & 13 & \pm & 6 & 0.47 \\
\hline \multicolumn{9}{|l|}{ Atto 550} \\
\hline Atto Rho6G & 189 & 83 & \pm & 17 & 42 & \pm & 7 & 0.51 \\
\hline Dy 549 & 231 & 6 & \pm & 3 & 1 & \pm & 1 & 0.34 \\
\hline Dy 549x2 & 240 & 11 & \pm & 4 & 4 & \pm & 1 & 0.46 \\
\hline Alexa 647 & 118 & 24 & \pm & 5 & 6 & \pm & 3 & 0.68 \\
\hline \multicolumn{9}{|l|}{ ATTO 620} \\
\hline \multicolumn{9}{|l|}{ Atto 633} \\
\hline \multicolumn{9}{|l|}{ Atto 647N } \\
\hline Atto 655 & 147 & 320 & \pm & 106 & 170 & \pm & 52 & 0.61 \\
\hline \multicolumn{9}{|l|}{ Dy 630} \\
\hline Dy 632 & 73 & 58 & \pm & 13 & 19 & \pm & 1 & 0.32 \\
\hline Dy 634 & 84 & 60 & \pm & 14 & 28 & \pm & 6 & 0.20 \\
\hline Dy 647 & 128 & 14 & \pm & 5 & 3 & \pm & 2 & 0.30 \\
\hline Dy 648 & 147 & 29 & \pm & 13 & 5 & \pm & 3 & 0.21 \\
\hline Dy 649 & 137 & 13 & \pm & 12 & 5 & \pm & 4 & 0.80 \\
\hline \multicolumn{9}{|l|}{ Dy 651} \\
\hline CF633 & 145 & 19 & \pm & 5 & 9 & \pm & 2 & 0.12 \\
\hline CF640 & 210 & 1 & \pm & 2 & 0 & \pm & 0 & 0.75 \\
\hline TF5 & 179 & 4 & \pm & 2 & 2 & \pm & 1 & 0.19 \\
\hline
\end{tabular}

\section{Negative control HeLa cells}

\begin{tabular}{|c|c|c|c|c|c|c|c|}
\hline Dye & Raw ph/spot & $\begin{array}{l}\text { Total } \\
\text { fram }\end{array}$ & & $\begin{array}{l}\text { spots } \\
40\end{array}$ & $\begin{array}{l}\text { Total } \\
\text { fram }\end{array}$ & $\begin{array}{r}\text { Spots } \\
120-140\end{array}$ & $\begin{array}{l}\text { Mobile spots fraction } \\
\text { frame } 20-40\end{array}$ \\
\hline Alexa 546 & 191 & 272 & \pm & 131 & 151 & \pm 112 & 0.44 \\
\hline Atto 532 & 178 & 21 & \pm & 6 & 7 & \pm 2 & 0.47 \\
\hline Atto 550 & & & & & & & \\
\hline Atto Rho6G & 208 & 108 & \pm & 35 & 37 & \pm 13 & 0.45 \\
\hline Dy 549 & 197 & 12 & \pm & 3 & 6 & \pm 2 & 0.23 \\
\hline Dy 549x2 & 189 & 10 & \pm & 5 & 5 & \pm 3 & 0.44 \\
\hline Alexa 647 & 61 & 63 & \pm & 47 & 12 & \pm 8 & 0.32 \\
\hline ATTO 620 & & & & & & & \\
\hline Atto 633 & & & & & & & \\
\hline Atto $647 \mathrm{~N}$ & & & & & & & \\
\hline
\end{tabular}




\begin{tabular}{|lllllllll|}
\hline Atto 655 & 147 & 172 & \pm & 20 & 88 & \pm & 11 & 0.44 \\
\hline Dy 630 & & & & & & & & \\
\hline Dy 632 & 63 & 149 & \pm & 95 & 35 & \pm & 23 & 0.35 \\
\hline Dy 634 & 75 & 28 & \pm & 11 & 4 & \pm & 2 & 0.34 \\
\hline Dy 647 & 98 & 187 & \pm & 99 & 47 & \pm & 16 & 0.42 \\
\hline Dy 648 & 230 & 31 & \pm & 7 & 9 & \pm & 2 & 0.21 \\
\hline Dy 649 & 92 & 6 & \pm & 3 & 2 & \pm & 1 & 0.37 \\
\hline Dy 651 & & & & & & & & \\
\hline CF633 & 141 & 32 & \pm & 18 & 13 & \pm & 6 & 0.53 \\
\hline CF640 & 175 & 3 & \pm & 2 & 1 & \pm & 0 & 0.32 \\
\hline TF5 & 230 & 11 & \pm & 4 & 4 & \pm & 2 & 0.15 \\
\hline
\end{tabular}

\section{Negative control MCF7 cells}

\begin{tabular}{|c|c|c|c|c|c|c|c|c|}
\hline Dye & Raw ph/spot & $\begin{array}{l}\text { Tota } \\
\text { fram }\end{array}$ & & $\begin{array}{l}\text { spots } \\
40\end{array}$ & $\begin{array}{l}\text { Tota } \\
\text { fram }\end{array}$ & & $\begin{array}{l}\text { spots } \\
0-140\end{array}$ & $\begin{array}{l}\text { Mobile spots fraction } \\
\text { frame } 20-40\end{array}$ \\
\hline Alexa 546 & 206 & 512 & \pm & 101 & 361 & \pm & 97 & 0.39 \\
\hline Atto 532 & 169 & 16 & \pm & 1 & 3 & \pm & 0 & 0.24 \\
\hline Atto 550 & & & & & & & & \\
\hline Atto Rho6G & 215 & 85 & \pm & 30 & 41 & \pm & 11 & 0.33 \\
\hline Dy 549 & 252 & 13 & \pm & 7 & 5 & \pm & 2 & 0.39 \\
\hline Dy 549x2 & 200 & 11 & \pm & 4 & 4 & \pm & 2 & 0.17 \\
\hline Alexa 647 & 116 & 23 & \pm & 12 & 13 & \pm & 11 & 0.71 \\
\hline ATTO 620 & & & & & & & & \\
\hline Atto 633 & & & & & & & & \\
\hline Atto 647N & & & & & & & & \\
\hline Atto 655 & 149 & 131 & \pm & 58 & 87 & \pm & 36 & 0.41 \\
\hline Dy 630 & & & & & & & & \\
\hline Dy 632 & 84 & 94 & \pm & 51 & 37 & \pm & 17 & 0.28 \\
\hline Dy 634 & 94 & 36 & \pm & 14 & 16 & \pm & 4 & 0.27 \\
\hline Dy 647 & 124 & 124 & \pm & 26 & 46 & \pm & 8 & 0.17 \\
\hline Dy 648 & 139 & 37 & \pm & 10 & 14 & \pm & 5 & 0.16 \\
\hline Dy 649 & 96 & 9 & \pm & 5 & 5 & \pm & 5 & 0.57 \\
\hline Dy 651 & & & & & & & & \\
\hline CF633 & 159 & 46 & \pm & 24 & 21 & \pm & 11 & 0.23 \\
\hline CF640 & 220 & 8 & \pm & 6 & 3 & \pm & 3 & 0.47 \\
\hline TF5 & 226 & 19 & \pm & 4 & 7 & \pm & 1 & 0.35 \\
\hline
\end{tabular}





\title{
Chapter 5
}

\section{The diffusion of single EGF receptors relates to their dimerization state and is altered by antagonists}

\author{
Peter J. Bosch ${ }^{1}$ \\ Johannes S. Kanger ${ }^{1}$ \\ Vinod Subramaniam ${ }^{1,2}$
}

1. Nanobiophysics, MESA+ Institute for Nanotechnology and MIRA Institute for Biomedical Technology and Technical Medicine, University of Twente, PO Box 217, 7500AE Enschede, The Netherlands

2. Present address: FOM Institute AMOLF, Science Park 104, 1098 XG Amsterdam, The Netherlands

This work entails collaborations with

Anton G.T. Terwisscha van Scheltinga

K.J.D. Kol

Thijs Oude Munnink

Steven de Jong

Elisabeth G. E. de Vries

affiliated at

Department of Medical Oncology, University of Groningen, The Netherlands 


\begin{abstract}
Although there is a strong link between dysregulation of the signalling of ErbB proteins and carcinogenesis, the response to current anticancer therapy based on ErbB antagonists is limited. This motivates the attempt to achieve a better molecular understanding of such antagonists to secure a more stable inhibition of ErbB signalling. Here, the diffusion characteristics of EGF receptor were determined by single-molecule-tracking, and shown to relate to the clustering state of the receptor by validation with established clustering conditions. In the presence of receptor antagonists, the diffusion of EGF receptors and ErbB3 on the cell membrane provided new insight into the clustering state of ErbB proteins. Based on our results and those in the literature, we propose the following model for EGFR dimerization. Normal ligand-induced back-to-back dimerization is only possible due to positive cooperativity between a weak connection through the extracellular dimerization arms and subsequent stabilization by strong connections between the kinase domains. Upon kinase inhibition with erlotinib or lapatinib the receptor changes conformation from the tethered to an alternative conformation. This conformation does not allow a stable dimer formation, also not upon ligand binding, possibly due to blocking of the binding sites in the kinase domains. Upon blocking of the ligand binding site of EFFR by cetuximab, the receptor changes its conformation to an alternative conformation, different from the conformation induced by kinase inhibition. This conformation allows a stable dimerization different from the signalling back-to-back dimer. We also obtained indications that upon cetuximab addition inactive ErbB3 goes from a pre-clustered to a more monomeric state, which is also more active to form dimers upon subsequent EGF addition. Single-molecule-tracking data might be able to explain and predict the response of tumour cells to recently applied combination therapies, such as cetuximab treatment in combination with an anti-ErbB3 treatment.
\end{abstract}




\subsection{Introduction}

Aberrant growth signalling pathways play a critical role in various types of cancers. The starting point for a vast network of signal transduction pathways is the binding of ligands to growth factor receptors in the plasma membrane of cells. The organization of these receptors in the plasma membrane shows dynamic clustering and hindered motion on the nano- and micro-scale levels. Molecular interactions, such as homo- and hetero-dimerization of receptors, and the phosphorylation state of single receptors, are also equally dynamic and are thought to play a crucial role in the amplification and sensitivity of signal transduction inside the cell ${ }^{1}$. The ErbB family of receptors is prototypical of receptor tyrosine kinases that are implicated in the development and progression of various human cancers, and are a widely studied target for drug discovery ${ }^{2,3}$.

A critical challenge to obtain more molecular insight is the difficulty to perform quantitative measurements and visualization, at these different length scales, of mechanisms such as ligandbinding, receptor homo- and hetero-dimerization, and the subsequent phosphorylation and triggering of the related signal transduction pathway. The ligand-induced dimerization and activation of the EGF receptor (EGFR) has been extensively studied over the years, which has led to a widely accepted molecular scheme underlying the biology of EGFR ${ }^{2,4}$. In short, ligand binding changes the tethered conformation of the extracellular part of the receptor to an extended confirmation, which allows two receptors to dimerize 5 . This homo- or hetero-dimerization leads to allosteric trans-activation of the kinase domains and phosphorylation of the tyrosine residues in the cytoplasmic tail ${ }^{6}$, which result in further signal propagation ${ }^{7,8}$. However, EGFR dimerization is not easily revealed by optical microscopy techniques such as by measuring Förster Resonance Energy Transfer (FRET), a way to detect protein interactions; only a few studies have shown FRET between EGF receptors ${ }^{9-12}$. Other more indirect techniques include image correlation ${ }^{13,14}$ and the related number and brightness approach ${ }^{15,16}$. To obtain further insights in the interactions and cluster size of these receptors at temporal and spatial resolution in live cells, other techniques are needed. Single molecule tracking microscopy is a unique method that can study this highly dynamic behaviour at a matching spatial resolution, and is especially well suited for application on cell membrane bound receptors ${ }^{17}$. This method has recently been applied to EGFR to study the influence of ligands and kinase inhibition ${ }^{18}$.

Receptor antagonists have been designed as anticancer drugs to suppress receptor signalling by specifically preventing certain molecular interactions with the receptor. Current molecular anticancer therapies such as humanized antibodies and kinase inhibitors targeting tyrosine kinase receptors show limited response, largely due to drug resistance through mutations or upregulation via gene amplification ${ }^{6,19}$. Combination therapy where more than one domain of the protein or even multiple proteins are simultaneously targeted seems to have most promises for therapeutic success $^{20}$. Whereas the presence of $\mathrm{T}$ cells around tumour cells is associated with better patient outcomes ${ }^{21}$, understanding the therapeutic effects of receptor antagonists to target upregulated receptors in tumour cells are hampered by the complexity of the immune system and molecular feedbacks in the live cell environment. Also knowledge about the early molecular effects such as receptor dimerization is limited. This makes it hard to understand the cellular response and predict long-term outcomes, yet might have high medical relevance. In addition, novel rationally designed antibodies are screened by cell proliferation ${ }^{22}$, but are not studied in the context of molecular effects within the signalling network of the receptor ${ }^{8}$. As knowledge of the sophisticated network mechanisms of signal cascades develops, the key to more robust therapeutic approaches may be revealed.

In this chapter we extend single-molecule tracking to study EGFR dimerization while inhibiting EGFR function with antagonists, and study the role of ErbB3 receptor herein. Specifically, we will verify whether the reduced diffusion of receptors can provide clues about their dimerization state, 
and study the reduced diffusion of EGFR and ErbB3 in the early events of EGFR targeting. Two classes of antagonists are currently used as anticancer drugs to target EGFR, kinase inhibitors and humanized antibodies. Kinase inhibitors are membrane penetrable small molecules, such as erlotinib and lapatinib, which bind the kinase domain of EGFR proteins. Humanized antibodies, such as cetuximab, block the extracellular ligand binding site of EGFR. We will study the altered diffusion of EGFR upon addition of these antagonists to obtain insight in the dimerization state of drug-targeted EGFR. To study these influences on otherwise unperturbed receptors, we utilize a small protein-tag labelling method which should have minimal influence on the receptor function.

\subsection{Background}

\subsubsection{Molecular interactions of ErbB receptors upon kinase inhibition and cetuximab}

The ErbB family consists of four closely related transmembrane tyrosine kinase receptors: epidermal growth factor receptor (EGFR; also known as ErbB1 or HER1), ErbB2 (HER2) which lacks a ligand binding site ${ }^{23}$, ErbB3 (HER3) which is a pseudo kinase ${ }^{24,25}$ and is believed to be only trans-activated (phosphorylated) by other ErbB receptors through dimerization, and ErbB4 (HER4) which is less well studied. Many reviews on the ErbB family are available, e.g. ${ }^{2,4-6}$. Dimerization of these receptors is mediated by domain II in the extracellular part of the protein (see chapter 1), and also other parts are involved ${ }^{10,26,27}$. Antagonists have been developed as anticancer drugs that block an ErbB receptor function by deregulating either the ligand-binding site (domain I and III), the extracellular dimerization arm (domain II), or the intracellular kinase accessibility. For example, erlotinib inhibits EGFR kinase ${ }^{28}$, and lapatinib is a dual kinase inhibitor for EGFR and ErbB2 ${ }^{29}$. Cetuximab binds domain III in the EGF-binding site in EGFR thereby sterically hindering ligand binding ${ }^{30}$. Other receptor sites are also targeted by anticancer drugs, such as domain IV of HER2 by trastuzumab. Remarkably, this site is not directly involved in dimerization $^{23}$, and the therapeutic effect of trastuzumab might therefore not be related to a molecular effect on the receptor signalling function. In this paper we focus on antagonists with a direct molecular effect on the function of EGFR (cetuximab, erlotinib, and lapatinib).

FRET and correlation microscopy have already revealed many details about the resting and liganded state of the receptor (listed in ref. 15). These findings suggested that resting EGFR is monomeric in physiological conditions, and can only reside in pre-formed (i.e. ligand independent) clusters in special conditions. Lowered temperatures (altering the lipid phase) and receptor overexpression orchestrate the formation of pre-clusters ${ }^{13,15}$. Pre-clustering is also one of the possible explanations for the intriguing high- and low-affinity ligand binding state of EGFR $^{31,32}$. Despite the wealth of current information regarding EGFR dimerization, many questions are still unanswered. Especially the molecular mechanisms involved in the influence of receptor antagonists on the normal receptor function remains elusive.

When the kinase domain of EGFR is inhibited, its signalling is strongly reduced, but the dimerization state of the receptor in that condition is not yet clear. The quinazoline tyrosine kinase inhibitor PD153035 and AG1478 were shown using immunoblotting to induce the formation of inactive EGFR dimers in the absence of ligand ${ }^{33,34}$. It was therefore suggested that tyrosine kinase inhibitors form a conformation distinct from the liganded back-to-back dimer ${ }^{35}$. However, Clayton et al. showed using FRET-FLIM that pretreatment of A431 cells (which have high endogenous expression of EGFR) with the same inhibitor (AG1478) caused unliganded EGFR to disassociate from pre-clusters and become monomeric ${ }^{9}$, although another possibility is that the receptor forms only heterodimers, since this would yield the same result in FRET measurements when only EGFR is labelled. Low-Nam et al. showed using single-particle tracking that when dimers of kinase inhibited EGFR were formed, they were not as stable as ligandinduced dimers of active receptors ${ }^{18}$, such that it is likely that most kinase inhibited receptors are 
monomeric. To explain unliganded receptor activation, Clayton et al. followed the model proposed by Zhu et al. ${ }^{27}$ that overexpression of EGFR results in pre-clustered oligomerisation which activates the kinase. They therefore assumed that upon inhibition of the kinase (resulting in inactivation) the receptor has to be in the monomeric state. Indeed pre-clustering has been shown to activate EGFR in some cases $^{36}$, but this does not necessarily mean that inactive dimers do not exist. In fact, pre-clustering using artificial dimerization domains (FKBP) did not activate the kinases of EGF receptor in $3 \mathrm{~T} 3$ cells $^{10}$. This cell type is devoid of endogenous EGFR, ErbB2, and $\mathrm{ErbB}^{37}$. In short, it remains an open question whether kinase inhibited EGF receptors are monomeric, which might be locked in an inactive conformation such that ligand addition would not dimerize them, or if they form alternative homo- or hetero-dimers with or without ligands $s^{9,35,38}$.

The oligomerisation state of EGFR upon cetuximab treatment is less well studied, as cetuximab is commonly believed to inhibit the extended conformation required for dimerization. However there are questions whether cetuximab is only preventing receptor activation by blocking the ligand binding site, or also sterically hinders the extracellular region of EGFR from making its conformational change to the extended configuration thereby preventing the normal dimerization pathway $^{30}$. Few reports are available whether alternative conformations might occur that result in inactive dimer formation, which is not unlikely as such formations are also proposed for kinase inhibition.

In recent years ErbB3 has been discovered as another important target for the development of ErbB related anticancer drugs ${ }^{39}$. Many advances in ErbB3 therapy have been made ${ }^{40}$, especially when ErbB3 therapy was combined with EGFR therapy. It was shown that this combined therapy has beneficial inhibitory effects and is more efficacious in multiple tumour models ${ }^{22}$. Although it has been suggested that ErbB3 is substituting the role of EGFR when therapeutic agents target that receptor, there is limited knowledge on the molecular state of ErbB3 in the early events of EGFR targeting. We therefore set out to find clues at the molecular level for why the activity of ErbB3 gets very potent upon EGFR targeting.

\subsubsection{Single molecule tracking}

With one-colour single-molecule microscopy it is possible to study the diffusion behaviour of single receptor proteins by measuring their trajectories, which may answer fundamental questions about the origin of motion of these protein $s^{41-44}$. When a protein interacts with other compounds (e.g. lipids, proteins or protein complexes), the formed complex will have a larger hydrodynamic radius resulting in slightly reduced diffusion. When a receptor protein forms dimers or interacts with kinases or adaptor proteins, it is therefore expected to have a lower diffusion speed than free monomeric receptor proteins. The diffusion coefficient reduces approximately by a factor $\sqrt{2}$ for a monomer to dimer transition, as theoretically the diffusion coefficient scales with the inverse radius of the transmembrane domain ${ }^{45}$. We first use conditions known to induce receptor dimerization to validate that the reduction in diffusion indeed correlates with dimerization. Reduced diffusion might then provide information about the dimerization of this receptor in unknown conditions. Moreover, the phosphorylation state of many tyrosine kinase receptors is often related to their ligand induced formation of dimers or higher order aggregates ${ }^{2,6}$. In the case of liganded tyrosine kinase receptors the dimerization and subsequent activation of the receptor coincides with reduced receptor diffusion, but the relation does not necessarily hold the other way round. In short, by analysis of the protein diffusion from recorded trajectories, information with respect to receptor interactions can be obtained. We use this information to answer questions about dimerization of EGFR under liganded and antagonistic conditions in live cells.

Single-molecule microscopy in two or more colours adds the possibility to directly observe dimerization of single receptors through correlated motion ${ }^{18,46}$. For homo-dimers this requires the labelling of the receptor species with two distinct colours, such that binding of two receptors can 
be observed at high resolution by a spatial overlap and correlated motion. Two receptors that have formed a dimer are expected to move together with correlated motion. Comovement analysis therefore offers a means to validate dimerization in trajectories recorded in two colours. By analysis of the dimerization events using software that detects comovement ${ }^{18}$, we will measure the lifetimes of the homo-dimerization events of EGF receptor in various conditions.

\subsection{Methods}

The microscopy setup is described in extensive detail in Chapter 2. Sample preparation is similar to the procedures described in Chapter 3 and 4, and differ only in the cell types used.

\subsubsection{Microscopy}

Briefly, measurements were performed on an inverted microscope with an Olympus PlanApo 100x/1,45 Oil TIRF objective. For the excitation we used a $532 \mathrm{~nm} 400 \mathrm{~mW}$ solid state laser from Pegasus Shanghai Optical Systems (Pegasus Lasersysteme, Wallenhorst, Germany), and a $637 \mathrm{~nm}$ $300 \mathrm{~mW}$ diode laser from Changchun New Industries Optoelectronics (Laser 2000, Vinkeveen, The Netherlands). The infrared light produced by the lasers was not sufficiently suppressed; we therefore filtered the green laser light with an FF01-543/22 (SemRock, Rochester, NY) filter and the red laser light with a D644/10m (Chroma, Olching, Germany) bandpass filter. The two laser beams were combined with a $610 \mathrm{~nm}$ longpass mirror. An achromatic quarter wave plate (VMTIM Optomechanische Werke, Jena, Germany) was used to obtain circularly polarized excitation light. An FF494/540/650-Di01 (SemRock) dichroic mirror separated the excitation light from the emission light. The emission light was filtered with an NF03-532/1064E (SemRock) and an NF02633 S (SemRock) notch filter, and then split with an FF640-FDi01 (SemRock) imaging flatness quality dichroic mirror. The emission light from the green excitation dyes was passed through a FF01-580/60 (SemRock) bandpass filter, and the light from the red excitation dyes through a HQ680/60m (Chroma) bandpass filter. The images were acquired using two Andor iXon EM+ DU-897 back illuminated EMCCD cameras. Using a calibration sample with grid lines separated at known distances, we determined that the pixel size was $119 \pm 2 \mathrm{~nm}$ (the magnification was $134 \mathrm{x})$. The sample was only illuminated during the acquisition time by utilizing the TTL output of the camera and TTL input of the lasers. Samples were heated to $35-37^{\circ} \mathrm{C}$ on the microscope with a stage heating plate and an objective ring heater.

Measurements were performed using TIRF illumination. The illumination time was chosen such that single molecules were clearly visible over the autofluorescence background of the cell (i.e. a mean of around $70-100$ photons per frame per molecule, see also Chapter 2). Videos were recorded at $24 \mathrm{fps}$, with a readout rate of $10 \mathrm{MHz}$ and frame size of $512 \times 512$ pixels. Each video recording consisted of 800 frames. Before recording each video, a minimal number of frames (approximately 10 to 30 frames) was used to focus on the basal membrane of the cell.

\subsubsection{Cell culture}

All cell culture materials were obtained from PAA Laboratories (Pasching, Austria) unless stated otherwise. The MCF7 epithelial human breast cancer cell line, and plasmids coding for SNAPEGFR and SNAP-ErbB3 were a gift from Jenny Ibach (Max Planck Institute, Dortmund, Germany). The H441 epithelial human lung adenocarcinoma cancer cell line, and FaDu epithelial human head and neck squamous carcinoma cells were a gift from Anton Terwisscha van Scheltinga (Department of Medical Oncology, University of Groningen, The Netherlands). MCF7 and $\mathrm{FaDu}$ cells were cultured in high glucose Dulbecco's Modified Eagle's medium (DMEM) supplemented with $10 \%$ FBS and Pen Strep at $37^{\circ} \mathrm{C}$ with $5 \% \mathrm{CO}_{2} . \mathrm{H} 441$ cells were cultured in Roswell Park Memorial Institute (RPMI) 1640 medium supplemented as above. 


\subsubsection{Sample Preparation}

Cells were plated in Greiner Bio CellView dishes with compartments of $1.9 \mathrm{~cm}^{2}$ (product \#627870) in full medium, and left overnight to adhere to the glass and grow to $40-60 \%$ confluence. The next day, the cells were transfected with SNAP-EGFR (or SNAP-ErbB3) with $0.5 \mu \mathrm{g}$ (FaDu and MCF7) or $0.15 \mu \mathrm{g}$ (H441) of SNAP-EGFR plasmid DNA in $0.5 \mathrm{~mL}$ Pen Strep free cell medium using 0.5 $\mu \mathrm{L}$ Lipofectamine LTX and $0.2 \mu \mathrm{L}$ Plus reagent (Invitrogen) for FaDu and H441 cells, or using 0.1 $\mu \mathrm{L}$ Effectene (Qiagen, Venlo, The Netherlands) for MCF7, as described in the suppliers' protocol. After 6 hours incubation with the plasmid, the cells were left overnight in normal medium to allow the cells to express the protein. On the next day the cells were starved in FBS free medium and left for another night to reduce the activity and internalization of the EGFR proteins. On the day of the experiment, cells were washed with starvation medium containing $0.5 \%$ BSA. Labelling of the SNAP-EGFR proteins was carried out thereafter by incubating the cells for 2 minutes $( \pm 10$ seconds) with $300 \mathrm{nM}$ of BG-Dy549 (SNAP-Surface 549, New England BioLabs, Ipswich, MA, USA) and 500nM of BG-CF633 (New England BioLabs, Ipswich, MA, USA) in starvation medium containing $0.5 \%$ BSA. Samples were washed after 2 minutes by replacing the labelling solution with PBS buffer supplemented with magnesium and calcium. This washing step was repeated at least three times

\subsubsection{Experiment}

Human recombinant epidermal growth factor (EGF) was obtained from PAN Biotech (Aidenbach, Germany). Cetuximab was a gift from the Academic Hospital Groningen (via Anton Terwisscha van Scheltinga, Department of Medical Oncology, University of Groningen, The Netherlands). Erlotinib and lapatinib were obtained from LC Laboratories (Woburn MA, USA). Dual-colour single molecule tracking experiments were performed on EGF receptor (labelled with the two dyes) in $5-10$ different cells for each receptor condition. Samples were kept at $35-37^{\circ} \mathrm{C}$ during measurements on the microscope.

Labelled serum starved cells were used directly to determine the diffusion of resting receptors. Subsequently, EGF or cetuximab was added to determine the diffusion of liganded or cetuximabbound receptors respectively $(+\mathrm{EGF}$, and $+\mathrm{mAB})$. Fluorescent microscopy recordings were acquired for 20 minutes after addition of the compound. In the case of cetuximab, after recording for 20 minutes, EGF was added, and recordings were acquired for another 20 minutes $(+\mathrm{mAB}$ + EGF). The kinase inhibitors (erlotinib and lapatinib) were added one hour before labelling. After labelling the cells, the inhibitors were also added to the PBS imaging solution. Recordings were acquired for 20 minutes, after which EGF was added, and recordings were acquired for another 20 minutes.

\subsubsection{Receptor tracking and correlated motion analysis}

Tracking software developed by others ${ }^{18,47}$ was used to obtain the trajectories from the microscopy recordings. Selection criteria were used to validate that signals from single molecules are individual emitting molecules. The settings used for these criteria and the cost matrices to perform the most likely tracking can be found in the appendix of Chapter 2. Subsequent dimer analysis was performed by comovement analysis of two receptors using Hidden Markov Models (HMM), which was developed by the same authors as the tracking software ${ }^{48}$; settings used can be found in the Supporting Materials.

\subsubsection{Diffusion state characteristics}

We fitted our data with a two-population dynamic diffusion system. To find the two diffusion constants $D_{1}$ and $D_{2}$ and the fraction $\alpha$ of the first (also called fast) population, we fitted a twopopulation cumulative distribution function (CDF) of squared displacements (SD) and squared 
two-step displacements for the complete set of trajectories ${ }^{49}$. In the fit of the CDF the two-step displacements are used to find the average localization accuracy $\sigma_{x y}$ (in one direction) and the unconvolved diffusion coefficient values $D_{1}$ and $D_{2}$ as follows. First we determined the convolved diffusion coefficients $\widehat{D}_{1}, \widehat{D}_{2}$ and $\alpha$ for the time lags $\tau$ corresponding to 1 and 2 frames:

$$
\begin{gathered}
C D F\left(\Delta R^{2}(1)\right)=1-\alpha_{\tau=1} \cdot \exp \left(-\frac{(\Delta R)^{2}}{4 \widehat{D}_{1, \tau=1}}\right)-\left(1-\alpha_{\tau=1}\right) \cdot \exp \left(-\frac{(\Delta R)^{2}}{4 \widehat{D}_{2, \tau=1}}\right) \\
C D F\left(\Delta R^{2}(2)\right)=1-\alpha_{\tau=2} \cdot \exp \left(-\frac{(\Delta R)^{2}}{4 \cdot 2 \widehat{D}_{1, \tau=2}}\right)-\left(1-\alpha_{\tau=2}\right) \cdot \exp \left(-\frac{(\Delta R)^{2}}{4 \cdot 2 \widehat{D}_{2, \tau=2}}\right)
\end{gathered}
$$

Since $\widehat{D}_{1, \tau=1}=D_{1, \tau=1}+{\sigma_{x y}}^{2}$ where ${\sigma_{x}}^{2}+{\sigma_{y}}^{2}=2{\sigma_{x y}}^{2}$, and similarly $2 \widehat{D}_{1, \tau=2}=2 \cdot D_{1, \tau=2}+$ $\sigma_{x y}{ }^{2}$, the estimated diffusion coefficient for the first (and similarly for the second) population corrected for the localization error is:

$$
D_{1}=2 \widehat{D}_{1, \tau=2}-\widehat{D}_{1, \tau=1}=\left(2 D_{1, \tau=2}+{\sigma_{x y}}^{2}\right)-\left(D_{1, \tau=1}+{\sigma_{x y}}^{2}\right)=2 D_{1, \tau=2}-D_{1, \tau=1}
$$

For the fraction $\alpha$ of the first population we take the average of the values $\alpha_{\tau=1}$ and $\alpha_{\tau=2}$.

We compared the diffusion coefficients found for the different receptor conditions. To find the chance that such conditions had a distinct diffusion coefficient, we performed a two-tailed t-Test assuming unequal variances of the two samples (Welch correction) in Origin (OriginLab, Northampton, MA, USA). Since we look for indications that there might be a difference in the diffusion coefficients and not for a definite proof, we regard differences with $\mathrm{P}<0.20$ for the null hypotheses (no difference) as significant. The P-value has to be compared in combination with the absolute difference of the two conditions.

\subsubsection{Diffusion state classification and visualization}

Diffusion state classification determines for each position within a measured trajectory the most likely diffusion state for a molecule given that its motion is described by a two-population diffusion system. The classification was performed using a gyration based classification scheme as described earlier (chapter 3) and in Fig. S1 in the Supporting Information.

Since the diffusion state classification requires a local measure of the diffusion of a molecule, the trajectories are split in small segments. Each segment contains a total number of $\mathrm{N}$ subsequent positions, and these segments must be classified to one of the two diffusion states obtained by the CDF fit, which yields the diffusion values of these states. Note that there is no need to determine the local diffusion value of the segment, only a need to classify the segment to one of the diffusion states. The segment length $\mathrm{N}$ is chosen such that the corresponding duration is shorter than the typical switching time between states, yet the segment length must be long enough to obtain an accurate measure for the classification. A gyration based classification was found to be both robust and accurate over a wide range of parameters (chapter 3). The use of the radius of gyration has been first proposed by Saxton to measure asymmetry in single molecule trajectories ${ }^{50}$, and it was demonstrated by Elliott et al. that it could also be used to detect confinement ${ }^{51}$.

The gyration based classification method uses a radius of gyration as a local measure of the diffusion. The value of the gyration radius is a measure of the space that is explored by the molecule within the segment, hence the radius will have a lower value for slow diffusion than for fast diffusion, and can be used as a differentiation criterion in classification. The gyration radius $R_{g}$ is defined as: 


$$
R_{g}=\sqrt{R_{1}{ }^{2}+R_{2}{ }^{2}}
$$

where $R_{1}$ and $R_{2}$ are the eigenvalues of the gyration tensor $T$ :

$$
\boldsymbol{T}=\left(\begin{array}{cc}
\frac{1}{N} \sum_{i=1}^{N}\left(x_{i}-\langle x\rangle\right)^{2} & \frac{1}{N} \sum_{i=1}^{N}\left(x_{i}-\langle x\rangle\right)\left(y_{i}-\langle y\rangle\right) \\
\frac{1}{N} \sum_{i=1}^{N}\left(x_{i}-\langle x\rangle\right)\left(y_{i}-\langle y\rangle\right) & \frac{1}{N} \sum_{i=1}^{N}\left(y_{i}-\langle y\rangle\right)^{2}
\end{array}\right)
$$

with $i$ enumerating all subsequent positions $\left(x_{i}, y_{i}\right)$ in a segment of length $N$. For the segment length we have chosen $\mathrm{N}=7$. When $\mathrm{N}$ was slightly altered (e.g. $\mathrm{N}=4$ ), we obtained similar state image maps (see below).

We applied this method as follows (Fig. S1). First, the diffusion constants $D_{1}$ and $D_{2}$ and the fraction size of the fast state from all displacements were determined using a CDF fit. Next we obtained the values of the gyration radius for all segments in every trajectory. If the radius $R_{g}$ of a given segment is smaller than a given threshold value $R_{\text {threshold }}$ the centre position of the segment is classified as slow diffusion and otherwise $\left(R_{g} \geq R_{\text {threshold }}\right)$ it is classified as fast diffusion. The threshold for classification $R_{\text {threshold }}$ is set at the fast fraction size percentile value of all the gyration radius values. This means that when the fraction of fast diffusion $\alpha$ is 0.71 , we take the threshold value such that $71 \%$ of the gyration radius values are higher than the threshold value. By taking this threshold we can perform the classification without a subjective threshold.

Image maps of diffusion states are composed by reconstruction of all the positions of all the molecules in one recording, yielding a map of the areas travelled by all the molecules. Each individual position is represented by a colour coded dot, with the colour representing the specific state found at that position and time: green for the fast state, and red for the slow state. This results in a colour coded super-resolution image showing the areas of slow and fast diffusion.

\subsection{Results}

We used SNAP-tag technology to fluorescently label EGFR with an intrinsically monovalent and photostable probe ${ }^{52-56}$. The SNAP-tag domain can be fused with a protein of interest, here EGF receptor, and allows for specific binding of fluorescent SNAP-substrates. This facilitates labelling of plasma membrane receptors in live cells with small and monovalent yet bright and photostable dyes favourable for single molecule tracking microscopy, and with the possibility to track receptors in multiple colours (chapter 4). By incubation with a straight-forward mix of two substrates with fluorescence emission at different colours, we systematically obtained equal levels of receptors labelled with green- and red-excitable fluorophores (Fig. 5.1D and Video 1), without a need to optimize expression levels. Labelling by means of protein tags has several advantages over other approaches, such as labelling with fluorescently labelled ligands and immunostaining with antibodies or Fab fragments. For example, the tag does not compete with ligand binding and has no antagonistic function, and therefore allows to study effects of ligands or antagonists on ligandfree and fully functional receptors ${ }^{57}$.

The EGF receptor is expressed in many tissues, but epithelial cells in particular depend strongly on this receptor for proliferation ${ }^{58}$. Therefore we looked at the role of ErbB1 in epithelial cells, and the role of its ligand EGF on the diffusion speed. Since it is known that room temperatures drives pre-clustering of the EGF receptor ${ }^{13,15}$, we performed all our experiments at physiological temperatures. 

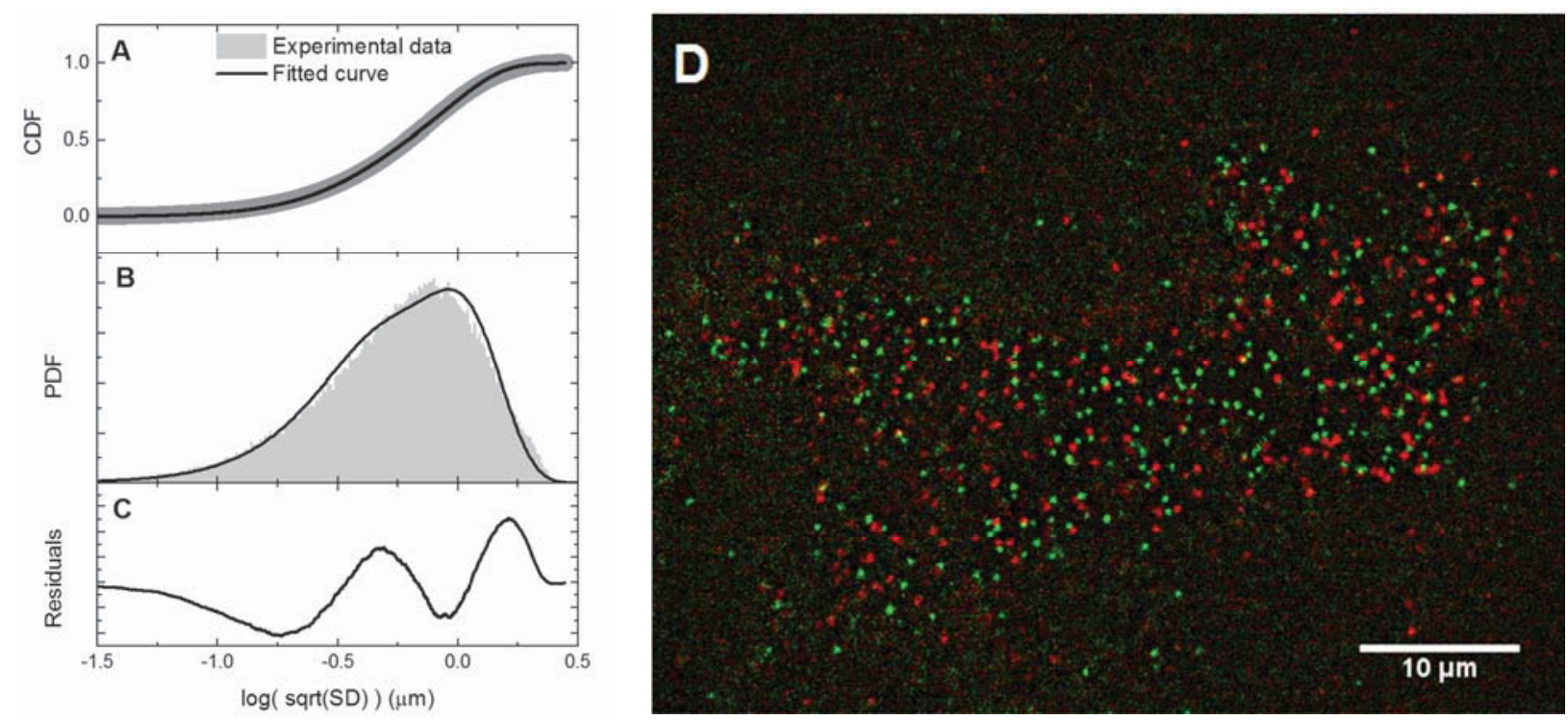

FIGURE 5.1 Example of diffusion populations fitting of resting EGF receptor in MCF7 cells. The fit shows that the motion of EGF receptor can be modelled as a two-population diffusion system. $(A)$ The cumulative density function (CDF) fit of squared displacements (SD) for the complete set of trajectories of the recording. $(B)$ The corresponding probability density function (PDF) of the squared displacements. $(C)$ Magnified residuals of the PDF show that the system is almost completely described by two diffusion states. $(D)$ An example of a single molecule fluorescence frame recording of resting EGF receptor labelled with two spectrally distinct fluorophores in MCF7 cells. The green channel contains emission from EGFR - Dy549, the red channel contains emission from EGFR - CF633.

The motion of plasma membrane resident receptor proteins can often predominantly be described by two dynamic populations (states) of Brownian diffusion ${ }^{18,59,60}$. Parameters defining the characteristics of such diffusion systems are the diffusion coefficients of either diffusion state and the lifetimes of the occupancy in both diffusion states. The diffusion coefficients $D_{1}$ and $D_{2}$ and the fraction of occupancy in one state $\alpha=\tau_{1} /\left(\tau_{1}+\tau_{2}\right)$ can be obtained by a fit to the cumulative density function $(\mathrm{CDF})$ of squared displacements ${ }^{49}$, and was performed for all receptor conditions.

\subsubsection{EGF induced dimerization and pre-clustering are reflected in the diffusion of EGFR}

Changes in the diffusion coefficient of the fast population $D_{1}$ are likely to reflect dimerization of receptors as detailed in the background (section 5.2.2). It is well established that unliganded EGFR is monomeric in cells that do not express a high number of EGF receptors, and forms dimers upon addition of its ligand. We therefore determined whether the diffusion coefficient of EGFR reflects its dimerization state in MCF7 cells, which express a low number of endogenous EGFR (Fig 3C). After incubation with $200 \mathrm{ng} / \mathrm{ml}$ EGF (more than the saturation dose for EGFR phosphorylation ${ }^{61}$ ) the average diffusion coefficient of the fast fraction of EGFR decreased significantly (Fig $3 \mathrm{~A}$ ) to a value that was $30 \%$ lower than that for monomeric EGFR. For resting (unliganded) receptors in the MCF7 cells measured we found $\overline{D_{1}}=0.148 \pm 0.006 \mu \mathrm{m}^{2} / \mathrm{s}$, and for liganded receptors $\overline{D_{1}}=0.112 \pm 0.006 \mu \mathrm{m}^{2} / \mathrm{s}$. This difference is significant $(\mathrm{P}<0.001)$. The intercellular spread of diffusion coefficients is probably due to the exact cell shape or cell cycle, and not due to difference in the monomer to dimer ratio. The average diffusion coefficients found compares to earlier values in literature from single-particle tracking of $\mathrm{EGFR}^{62,63}$, but is about three times faster compared to single-particle tracking experiment using quantum dots ${ }^{18}$; the large 
size of quantum dots might have slowed down the diffusion of these receptors ${ }^{64}$. By monitoring the diffusion coefficient of one receptor, we cannot tell whether EGFR forms homo- or heterodimers. Dimerization of EGFR in MCF7 cells is also possible as hetero-dimers with ErbB2, as MCF7 cells have an elevated number of ErbB2 (Fig. 5.2C).

Pre-clustering of unliganded EGF receptors is a complicating factor in the direct relation of EGF-induced dimerization and its diffusion coefficient. The degree of pre-clustering was found to be more prevalent in cells expressing the receptor at a high level ${ }^{15}$. To validate whether such subtle effects of partly monomeric and partly pre-clustered receptors are reflected in receptor diffusion, we also measured the receptor diffusion in H441 cells, a non-small cell lung cancer (NSCLC) line, which have a much higher number of endogenous EGF receptors than MCF7 cells (Fig. 5.2C). The pre-clustering indeed coincides with a reduced diffusion of EGFR compared to monomeric receptors in resting MCF7 cells $(\mathrm{P}=0.06$, Fig. $5.2 \mathrm{~A})$. The unliganded receptors in $\mathrm{H} 441$ cells have a diffusion coefficient $\left(D_{1}=0.132 \pm 0.006 \mu \mathrm{m}^{2} / \mathrm{s}\right)$, which is not as low as complete dimerization induced by EGF in the same cell line $\left(D_{1}=0.114 \pm 0.007 \mu \mathrm{m}^{2} / \mathrm{s}, \mathrm{P}=0.08\right)$.
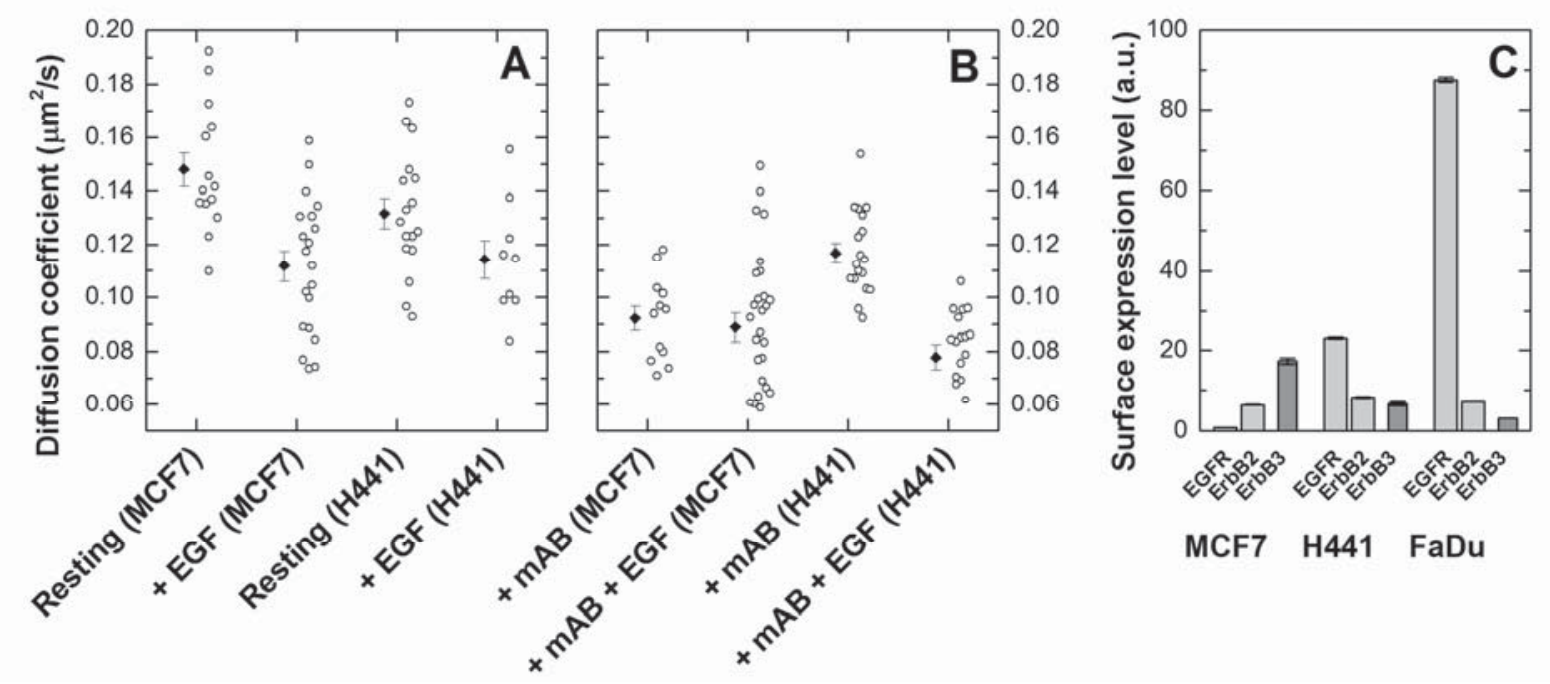

FIGURE 5.2 Diffusion coefficient (fast fraction) of EGF receptor in MCF7 and H441 cells under different conditions. The open circles are the diffusion coefficients found in the microscopy recordings for different cells measured in one condition. The closed circle shows the mean and the standard error of all recordings. $(A)$ The diffusion coefficient of EGFR decreases in MCF7 cells when unliganded receptors are exposed to their ligand EGF. Unliganded EGFRs in H441 cells have a lower diffusion coefficient compared to MCF7 cell, but upon EGF addition they slow down as much. $(B)$ The diffusion coefficient of EGFR slows down when exposed to cetuximab (mAB) in MCF7 cells, but only marginally in H441. Upon EGF addition, cetuximab bound receptors did not further slowdown in MCF7 cells, but did in H441 cells. (C) The surface expressions of EGFR, ErbB2, and ErbB3 in the different cell lines used in this study measured with flow cytometry using cetuximab (EGFR), trastuzumab (ErbB2), and SGP1 (ErbB3) as primary antibodies. Since a different secondary antibody was used for ErbB3, its expression level cannot be directly compared with the level of EGFR and ErbB2. (The data in panel C was contributed by K.J.D. Kol).

\subsubsection{The slow population of EGF receptor is transient and is not influenced by ligand binding}

The slow population with diffusion coefficient $D_{2}$ was almost immobile $\left(<0.01 \mu \mathrm{m}^{2} / \mathrm{s}\right)$ both for unliganded and liganded receptors (Fig S2), which is about an order of magnitude slower compared to the diffusion of the fast population. To find out whether the multiple diffusion populations were originating from two distinct pools of fast molecules and very slow molecules, or from a pool of molecules transiently making transitions between the states, we applied diffusion 
state classification on the trajectories measured. We found that EGFR transiently switches from the fast (mobile) state to a very slow (almost immobile) state (Video 2). Therefore the slow diffusion state is not related to an interaction with another single protein (homo- or heterodimerization), but more likely with transient visits to cytoskeletal structures or large protein clusters. For example, they could be receptors clustering in clathrin-coated pits, the main region for endocytosis ${ }^{8,65}$. To find out whether the very slow diffusion state became more visited upon ligand addition, we determined its fraction $(1-\alpha)$. The data showed that both unliganded receptors and receptors with ligand visited the very slow diffusion state (Fig S3). This is in contrast with the report that receptors showed dynamic transitions to a very slow diffusion state only upon EGF addition ${ }^{63}$, but in agreement with other reports ${ }^{48}$ (and personal communications with D. Lidke and C. Valley).

\subsubsection{Cetuximab induces dimerization of EGFR}

To study the reduced diffusion of cetuximab bound EGF receptors, we added $500 \mathrm{nM}$ cetuximab to resting MCF7 and H441 cells. This dose is much higher than the concentration where phosphorylation of EGFR is maximally inhibited (10nM, IC50 is $0.3-1 \mathrm{nM}$ in NSCLC cell lines ${ }^{66}$ ) to ensure rapid binding. In MCF7 cells the diffusion of resting EGFR went down upon cetuximab binding to values even lower than EGF-induced dimerization (Fig. 5.2B). The exact values for the mean and standard error for each receptor condition can be found in Table S1 in the Supporting Materials. The difference in diffusion between the resting receptors and the cetuximab bound receptors is significant $(\mathrm{P}<0.001)$. Subsequent $\mathrm{EGF}$ addition did not further reduce the diffusion of EGFR, and if so not by much. These observations indicate that cetuximab induced an almost complete ligand-independent dimerization of the receptor population.

The results were slightly different for EGF receptors in an EGFR-rich environment (Fig. 5.2B,C). In $\mathrm{H} 441$ cells the diffusion of resting EGFR went down marginally upon cetuximab binding $(\mathrm{P}=$ $0.02)$, whereas subsequent EGF addition did further slowdown the receptors $(\mathrm{P}<0.001)$ again to even lower values compared to EGF-induced dimerization $(\mathrm{P}=0.001)$. This indicates that the monomeric EGF receptors in an environment of pre-clusters do not dimerize upon cetuximab addition, perhaps due to that cetuximab does not (strongly) bind to EGFR in a pre-clustered formation.

\subsubsection{An active kinase domain is essential for stable dimerization of EGFR}

There are contradicting reports in the literature whether inhibition of the kinase domain of EGFR results in monomeric or dimerized receptors. We investigated the diffusion of EGFR upon addition of the tyrosine kinase inhibitors erlotinib (specifically targeting EGFR) and lapatinib (targeting EGFR and ErbB2) to obtain more insight in the elusive role of the kinase domain of EGFR in dimerization. A 24 hour incubation with kinase inhibitors will negatively affect normal cell proliferation. We therefore incubated MCF7 cells for one hour with $10 \mu \mathrm{M}$ erlotinib or with $20 \mu \mathrm{M}$ lapatinib, doses that are just above the IC50 values for erlotinib and lapatinib ${ }^{67}$. We used MCF7 cells to look whether resting monomeric EGFR (no pre-clustering) still underwent ligandinduced dimerization.

We did not observe diffusion changes of EGFR between its resting state and after treatment with one of both kinase inhibitors (Fig. 5.3). This supports the hypothesis that kinase inhibited unliganded EGFR is monomeric, as suggested earlier ${ }^{9,10,18}$, but contrary to other reports ${ }^{33-35}$. We also observed that subsequent ligand addition to the kinase inhibited EGFR did not induce reduced diffusion of EGFR. This supports that ligand addition to kinase inhibited EGFR does not result in (further) dimerization, which was also observed in kinase dead and tyrosine residues mutated EGFR ${ }^{10,63}$. As ligands can still bind the extracellular part of the kinase inhibited receptors, which normally induces a back-to-back dimer, we conclude that the receptor makes a conformational change which prevents this dimerization. 


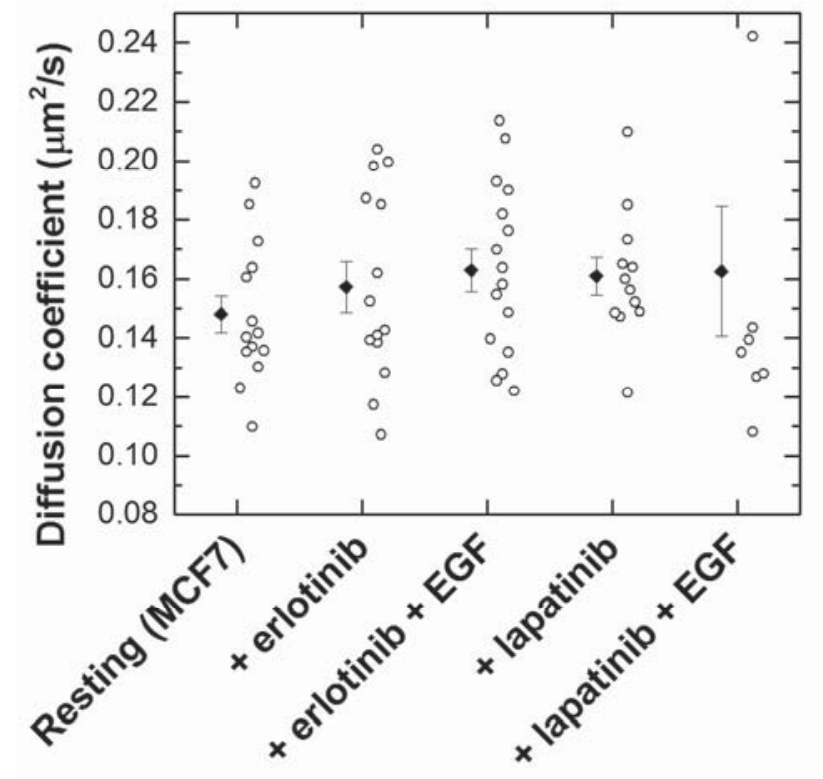

FIGURE 5.3 Diffusion coefficient (fast fraction) of EGF receptor in MCF7 cells under different receptor conditions in the presence of kinase inhibitors. The open circles are the diffusion coefficients found in the microscopy recordings for different cells measured in one condition. The closed circle shows the mean and the standard error of all recordings. In the presence of kinase inhibitors the diffusion coefficient of EGFR does not slowdown and ligand-induced slowdown is prevented.

\subsubsection{ErbB3 is removed from preformed clusters upon cetuximab treatment but not upon EGF stimulation}

It is not clear why some cells respond better to cetuximab treatment in combination with an antiErbB3 treatment ${ }^{22}$. When ErbB3 is indeed taking over the signalling role of EGFR as proposed ${ }^{68}$, deactivating EGFR with cetuximab treatment should then make ErbB3 become more accessible for dimerization with receptor partners. In that case, an additional anti-ErbB3 treatment would indeed help to obtain a better response to therapy. We chose to take the FaDu cell line, which is benefiting from such a combination therapy; proliferation of $\mathrm{FaDu}$ cells was found to be worse when cetuximab is combined with anti-ErbB3 treatment ${ }^{22}$. We expressed and fluorescently labelled SNAP-ErbB3 receptors in a comparable manner as EGFR, and monitored the diffusion coefficient of ErbB3 in FaDu cells before and after 500nM cetuximab treatment (Fig. 5.4).

The relatively slow diffusion of ErbB3 in resting cells indicates a pre-clustered state, which corresponds to an earlier report that suggests that inactive ErbB3 is a highly clustered preassociated receptor ${ }^{69}$. Without inhibition of any ErbB receptors, EGF binds to EGFR which subsequently forms dimers with EGFR or HER2 but not ErbB3 ${ }^{70}$. Indeed we did not observe diffusion changes of ErbB3 receptors upon addition of EGF. Interestingly, upon cetuximab treatment we observed a dramatic increase in the diffusion of ErbB3 $(\mathrm{P}=0.001)$, even though there is no direct interaction of cetuximab to ErbB3 as far as we know. This result indicates that a large portion of the pool of ErbB3 receptors leave the highly clustered state and become monomeric or dimerized. A subsequent stimulation by EGF showed a small decrease in the diffusion coefficient of ErbB3 $(\mathrm{P}=0.11)$. This could be an indication that ErbB3 is binding a partner when in an environment of inhibited EGFR and might form a stable dimer. The combination of results indicates that ErbB3 is indeed entangled with the signalling role of EGFR, but only when EGFR is treated with cetuximab. 


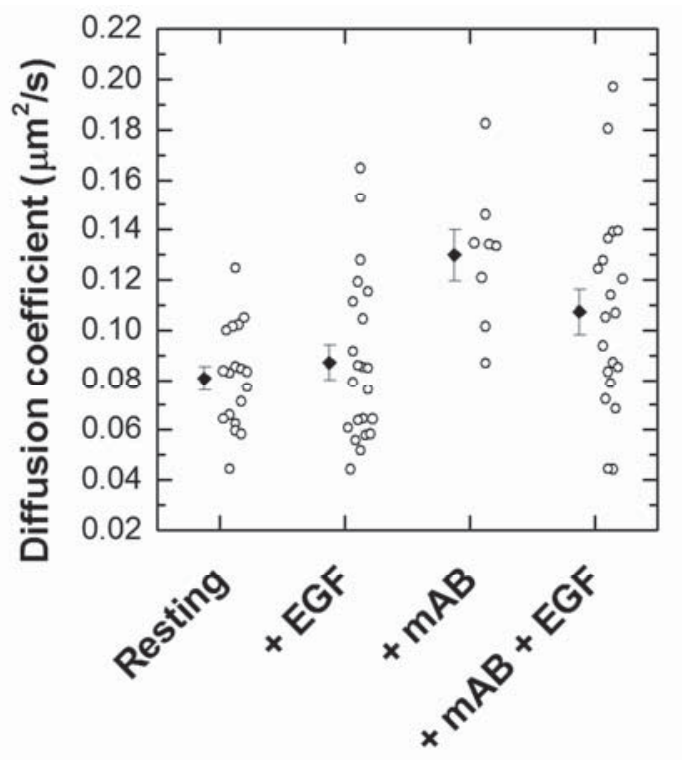

FIGURE 5.4 Diffusion coefficient (fast fraction) of ErbB3 receptor in FaDu cells. The open circles are the diffusion coefficients found in the microscopy recordings for different cells measured in one condition. The closed circle shows the mean and the standard error of all recordings. EGF addition does not influence the resting ErbB3 receptor. Upon cetuximab treatment the ErbB3 receptor becomes more mobile, and subsequent EGF addition slows the receptor down slightly.

\subsubsection{The spatial organization of regions where receptors transiently experience strong membrane interactions are independent of the receptor dimerization state}

Apart from monitoring the two diffusion coefficients (of the two diffusion states), the spatial distribution of the slow diffusion state (population) of a receptor protein can provide additional insight in the mechanism of receptor signalling or its lifespan. For example it can yield information about potential internalization hotspots or the role of cytoskeletal structures, since strongly retarded diffusion (the slow population) of a protein is very likely related to strong interactions with the protein. We developed a diffusion classification scheme (chapter 3), which can reveal where on the membrane a receptor protein is residing in the fast and where in the slow diffusion state. We analysed the single-molecule trajectories to classify the motion of unliganded EGF receptors (Fig. 5.5A) and liganded receptors (Fig. 5.5B) in MCF7 cells. We observed that there are distinct zones of slowed diffusion, although these zones are independent of the ligand occupancy of the receptors. We also see filopodia at the boundaries of the cell, and possibly collapsed filopodial structures on the basal membrane. Since EGF receptor undergoes retrograde transport in filopodia ${ }^{70}$, its presence in filopodia is expected. We furthermore observed that EGFR seems to be more present in the periphery of the cell adjacent to other cells than in the basal membrane adjacent to the cover glass, which is commonly observed for EGFR ${ }^{71}$.

\subsubsection{Direct dimerization observations using single molecule tracking conflicts with high levels of endogenous receptors}

We did not directly observe dimerization using comovement analysis ${ }^{18}$ other than events with very short dimer lifetimes (Fig. 5.6A,B). Such short events are probably not actual dimers but two proteins that are just very close together coincidentally. To determine the expected durations of receptors which get close together and falsely appear to be a dimer, we performed simulations in a simulation area of $60 \times 60 \mu \mathrm{m}$ where 1,000 molecules experienced motion described by a twostate diffusion system defined by the same characteristics as found for EGFR (Fig. 5.6F): $D_{1}=0.10$ $\mu \mathrm{m}^{2} / \mathrm{s}, D_{2}=0.01 \mu \mathrm{m}^{2} / \mathrm{s}, \tau_{1}=300 \mathrm{~ms}, \tau_{2}=300 \mathrm{~ms}, \sigma_{x y}=30 \mathrm{~nm}, 24 \mathrm{fps}$; subsequently comovement analysis was performed on these simulated trajectories. The lifetimes detected for liganded receptors were not different from the lifetimes obtained in simulations for receptors that only show comovement coincidentally (Fig. 5.6D). 


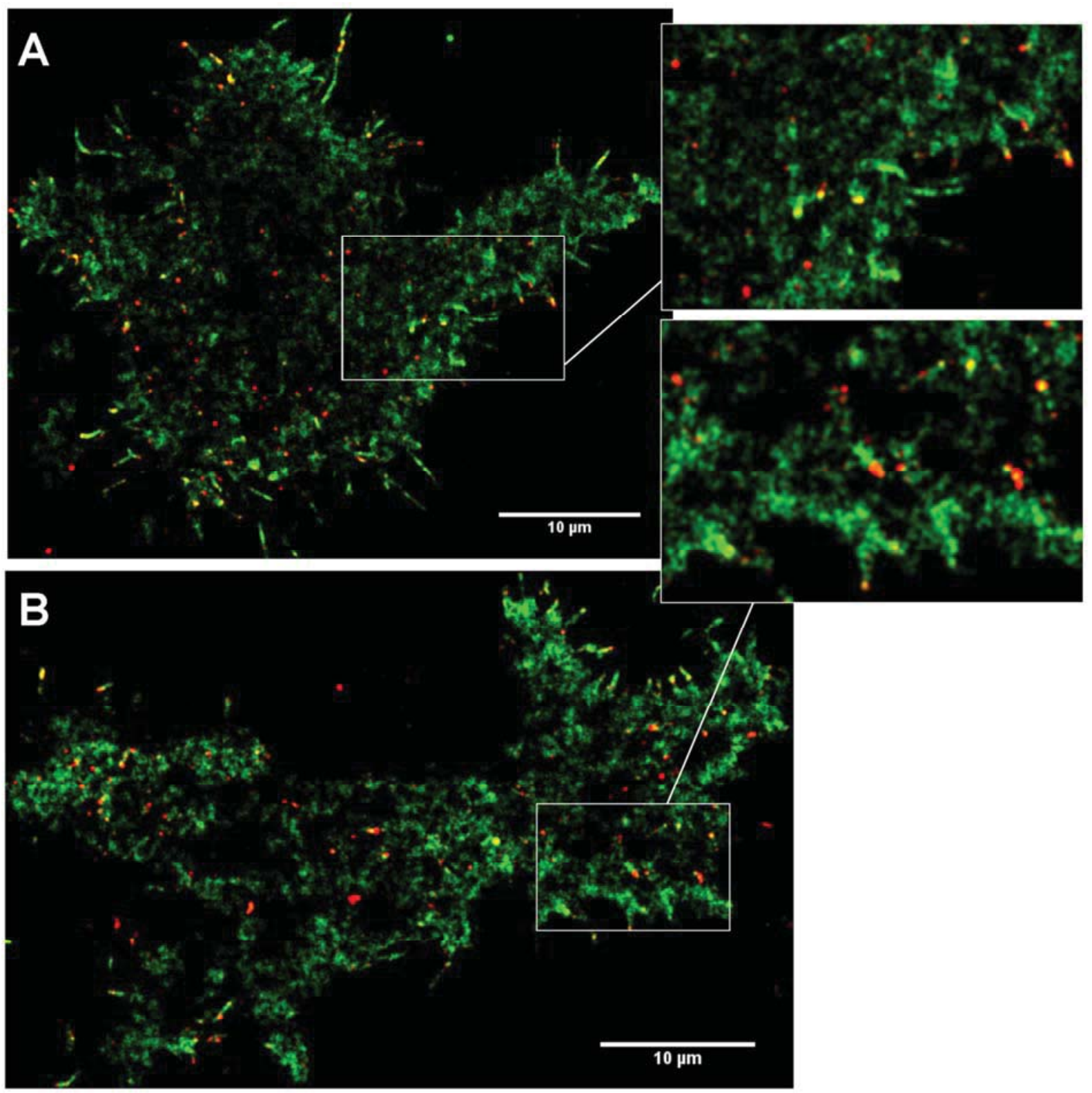

FIGURE 5.5 The spatial distribution of the two diffusion states exhibited by unliganded $(A)$ and liganded $(B)$ EGF receptors in an MCF7 cell. The reconstructed image shows the positions where the motion of a receptor was classified as fast diffusion in green, and positions where the motion was classified as slow diffusion in red. For regions at the periphery of the cell the zoomed images show cellular structures, such as filopodia. The resolution of the reconstructed image is $60 \mathrm{~nm} /$ pixel.

Whereas others have seen long-lasting dimers for the same receptors in $\mathrm{CHO}$ and HeLa cells ${ }^{48}$, we used MCF7 cells, which do not express much endogenous EGFR, but express ErbB2 at a number of approximately 100,000 receptors. This high number of non-labelled dimerization partners could easily explain why we did not observe dimer events. In fact, a dimer would only be visible when one of the receptors was EGFR- Dy549 and the other EGFR - CF633. The chance $p_{1}$ to see a labelled receptor form a dimer with a differently labelled receptor in an environment with numerous unlabelled receptors is very slim. For example, when 100,000 ErbB receptors (e.g. EGFR or ErbB2) endogenously expressed by a cell (cell lines used here have this level or higher) are dimerizing in a completely random fashion, and we have transfected and labelled 100 receptors with one dye and 100 receptors with a spectrally distinct dye, the chance $p_{\text {dimer }}=(100 / 100,200) \approx$ 
0.001 . Even when all 200 labelled receptors get dimerized, the chance $p_{1}$ to see one dimer is still $\operatorname{slim}\left(p_{1} \approx 200 p_{\text {dimer }} \approx 0.2\right)$, and to observe two dimers is almost zero $\left(p_{2} \approx\left(200 p_{\text {dimer }}\right)^{2} \approx 0.04\right)$. This means that when ideal non-blinking labels could be used, an average of 5 recordings are needed to observe one dimer, which is impractical to compose statistics about dimer events. Therefore to observe dimers by correlated motion analysis clearly a cell line is needed that has a limited expression level of endogenous ErbB receptors.
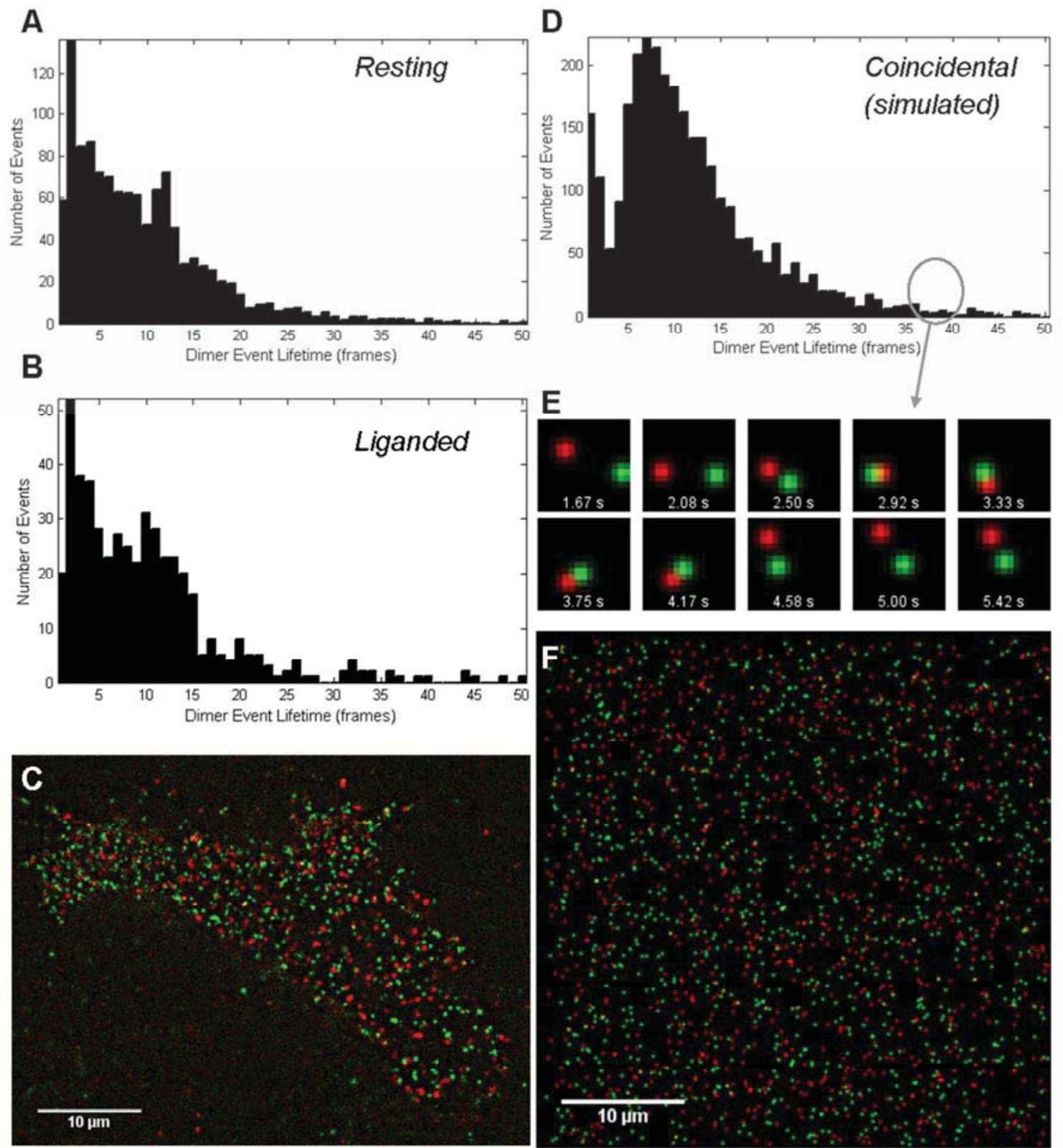

FIGURE 5.6 Dimer lifetimes were detected with comovement analysis. The lifetimes detected for EGFR are not different from the lifetimes obtained in simulations for two receptors that show correlated motion coincidentally. (A) Histogram of dimer lifetimes for resting (unliganded) receptors. (B) Histogram of dimer lifetimes for liganded receptors. The total number of molecules analysed is different, hence the number of events too; only the shape of the histogram is informative. (C) Example of a two-colour image frame with liganded receptors in an MCF7 cell as used for comovement analysis. (D) Histogram of dimer lifetimes for coincidental comovement in simulations with a dynamic diffusion system set at the same characteristics as found for EGFR. (E) Image montage to show an example of a coincidental encounter of two receptors registered as a dimer with a lifetime of 1.5 seconds. (F) Frame from the simulation performed to show that the concentration of molecules was about equal in the experimental and the simulated data. 


\subsection{Discussion}

We speculated that the reduced diffusion observed is due to dimerization events. However, different underlying causes can be postulated that lead to reduced diffusion speeds, such as altered presence in corral regulated microenvironments ${ }^{72}$. Since our two-colour tracking experiments (which allows direct observation of dimers through co-movement analysis) did not show dimerization events, it is also possible that the reduction of the diffusion coefficient represents monomers binding to other interaction partners or represent oligomerisation in higher-order clusters. Nevertheless, reduced diffusion of receptors in cells are related to a molecular interaction with the receptor, otherwise no difference would be observed. Since other interactions with larger molecules that may induce similar diffusion reductions are unknown, we attributed the reduced diffusion speed to an interaction with one of the receptor partners, hence dimerization.

While we used a two-state diffusion system to describe the motion of receptors, a three-state system to describe the immobile (slow), dimeric (reduced), and monomeric (fast) states of the receptor might be more correct. However, ignoring the immobile state, the reduced diffusion of one state in a two-state model, or a change of occupation between two states in an three-state model, are equivalent. For example, a 50\% dimerization will give a diffusion coefficient value equal to the average of the diffusion coefficients of the purely dimeric and monomeric state. We therefore chose to use the easiest model applicable to describe our data.

Certainly, the relation between diffusion and dimerization is complex, and absolute diffusion coefficients cannot be used as the only criterion for identifying dimers and activity, as also stressed by others ${ }^{18}$. Ligand binding, conformational change, dimerization, phosphorylation of receptors, recycling, and internalization are all influencing the highly complex interplay and dynamic cooperativity of transiently formed dimers ${ }^{73}$, and inactive dimers exist in a wide variety ${ }^{26,38}$. We therefore verified that the relation between reduced diffusion speed and dimerization was indeed valid for several established receptor conditions. Receptor dimerization under other less established (antagonistic) conditions for EGFR could be explained in the context of existing reports and models. The reduction of the diffusion speed of EGFR upon ligand-induced dimerization was also observed in two-colour tracking experiments and correlated with the lifetime of directly observed dimers (comovement), both for wild-type and kinase inhibited receptors ${ }^{18}$. The same reports showed using comovement analysis that kinase inhibited receptors form dimers upon addition of ligand, which coincided with a reduction of the diffusion speed, whereas we did not observe such effects. Future investigations to confirm dimerization under antagonistic receptor conditions could include autoradiography and immunoblotting as performed earlier ${ }^{33,34}$, although the harsh procedures and prolonged incubation at cold temperatures might not represent the true oligomerisation state of the receptor in live cells.

We measured the different receptor conditions within seconds after addition of a compound, for a period of 20 minutes. We believe that the receptors are in a steady state after a few seconds with the high concentrations of ligand and antagonists used. In comparison, it is known that ligand induced phosphorylation happens within seconds ${ }^{7}$ and is maintained for hours ${ }^{74}$. The 20 minutes timeframe was taken since ligand induced dimerization is maintained for approximately 20 minutes, after which receptors probably dissociate or degrade ${ }^{10}$.

Inactive dimer formation upon cetuximab addition might seem an elusive arrangement, since ligand-induced dimerization is normally associated with subsequent kinase activity. Cetuximab addition does not lead to tyrosine kinase activation of ErbB proteins ${ }^{75}$. However, artificially formed wild-type EGFR dimer arrangements have been shown to be inactive as well ${ }^{10}$. Upon ligand binding a conformational change in the extracellular part of the ErbB protein occurs to allow the dimerization domain to become accessible for receptor back-to-back dimerization. Since cetuximab blocks the ligand binding site in the tethered state of EGFR, it is believed that the conformational change cannot take place due to steric clashes, thereby preventing the normal 
back-to-back dimerization and ultimately the activation of EGFR ${ }^{30}$. It is therefore tempting to explain the slowed diffusion as the formation of an alternative side-by-side and/or head-to-head dimer proposed in earlier reports ${ }^{9,35,38}$. It will therefore be interesting to measure whether cetuximab bound EGFR remains in the tethered state and indeed does not undergo a conformational change to the extended or an alternative state. This can be verified by measuring FRET between a dye at the top of EGFR and a membrane-bound dye, as demonstrated recently for the EGF induced conformational change ${ }^{76}$. The therapeutic value to understand the exact arrangement formed upon cetuximab binding is that such inactive formations may be locked (sequestration) by another antibody which has synergetic anticancer effects, in the same way as described for the configuration formed after AG1478 treatment ${ }^{35}$.

The direct inhibition of EGFR activation through blocking its ligand binding site and therefore dimerization is believed to act as the primary mechanism for the anticancer activity of cetuximab $^{75}$. Therefore drug-induced dimerization through an alternative arrangement can be considered to be a weak aspect for secure inhibition of the receptor activation, since the receptors are primed for activation due to their close proximity. The anticancer activity of cetuximab is however also attributed to additional mechanisms such as cellular cytotoxicity through the immune response and receptor downregulation through internalization. It is therefore interesting to correlate the further lifespan of the receptors after the initial molecular responses, such as the internalization rate and the expression level.

We did not expect dramatic changes in the dimerization state of ErbB3 upon cetuximab treatment. Our results indicate that ErbB3 is removed from preformed clusters upon inhibiting EGFR and becomes more accessible for other receptors. This means that an additional anti-ErbB3 treatment in therapies aimed at EGFR (using cetuximab) can be expected to have beneficial response. While this was observed to be the case in FaDu cells, other cell lines might have different responses, which can be tested using the same methodology.

There is a discrepancy in earlier results about the influence of kinase inhibition to the clustering state of EGFR. Some reports showed that kinase inhibition increases the formation of inactive dimers $^{33,34}$. In contrast to that, an active kinase domain was shown to be required for dimerization $^{63}$, even when the extracellular dimerization arm is intact. Others reported more direct evidence that kinase inhibition caused EGFR to disassociate from pre-clusters and become monomeric ${ }^{9}$ or at least does not further cluster the receptor ${ }^{10}$ and reduces the lifetime of this dimer $^{48}$. Also mAb 806 antibody, which binds domain II (dimerization arm) of EGFR exclusively in the untethered conformation ${ }^{77}$, showed increased reactivity upon kinase inhibition ${ }^{35}$. Based on the diffusion speed, we believe that kinase inhibited EGFR in the cell systems tested is not partly pre-clustered as indicated by anisotropy measurements ${ }^{10}$. This inconsistency might originate from the different ways to achieve kinase inhibition (small molecule inhibitors versus receptor mutations). Our results favour that kinase inhibition keeps the receptor in their monomeric state, and also indicate that subsequent ligand addition does not affect the monomeric state of EGFR (Fig. 5.3).

In the light of the above discussion, we postulate the following model for the influence of kinase inhibition on EGFR (Fig. 5.7). Upon binding of the kinase inhibition on the kinase domain of the receptor, a conformational change has to take place since EGF does not lead anymore to the normally induced dimerization of the receptor. This conformational change is from the tethered to an alternative but not extended conformation, since this would allow (weak) dimerization. An alternative conformation was already proposed earlier due to exclusive binding of $\mathrm{mAb} 806$ to an untethered conformation of the receptor ${ }^{77}$. Even though the extracellular dimerization arm might be exposed, as well as the ligand binding site, no dimers of any arrangement are stably formed, because the kinase domain is mainly responsible for stable dimerization, and binding to this domain is blocked. Therefore the alternative conformation is probably orchestrated by the kinase domain. 


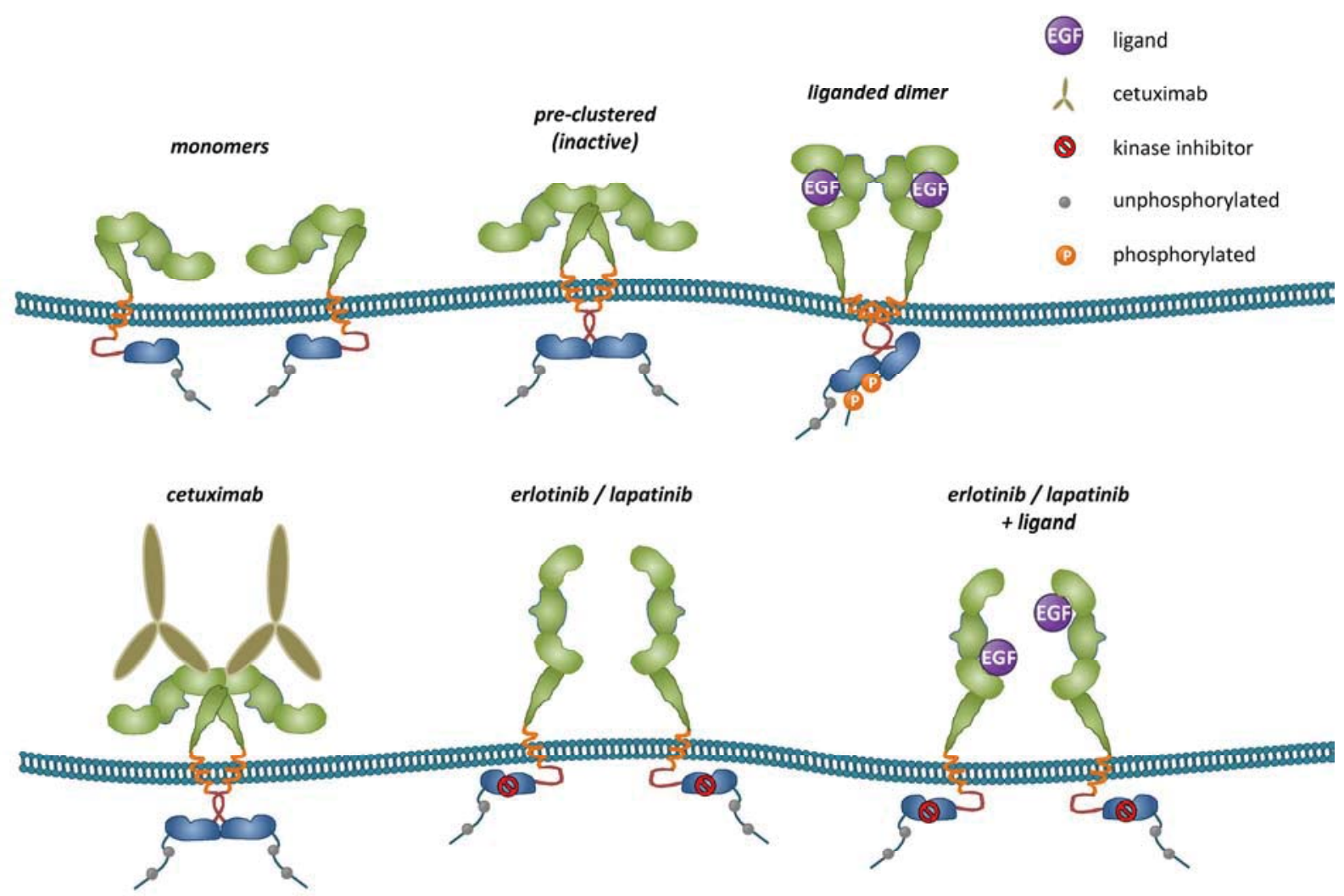

FIGURE 5.7 Model of the dimer formations of EGFR and the influence of cetuximab and kinase inhibitor therein. Inactive unliganded receptors are monomeric. High concentrations of receptors or certain lipid phases can however lead to pre-clustering, which is an inactive dimer conformation where the kinase domains cannot phosphorylate the tail of the receptor partner. Liganded receptors undergo an extracellular conformation change, which drives the kinase domains to stably bind each other and phosphorylate at least the tail of one partner. Cetuximab inhibited receptors can form dimers with an alternative extracellular confirmation, but does not allow the kinases to stably bind and phosphorylate a receptor tail. When the kinase domain is inhibited, the weak extracellular dimerization might still be operative, but stable dimers cannot be formed because the kinase domains cannot bind each other.

Our model builds upon other hypotheses that the dimerization arm is needed for dimer stabilization but that the kinase part is mainly responsible for receptor dimerization ${ }^{63}$. It also agrees with recent findings that the kinase domain on its own can form stable dimers but is impeded to do so by the tethered conformation of the extracellular and transmembrane domain $^{78}$, such that both extracellular and intracellular domains are involved in dimerization ${ }^{27}$. Following this model, the normal ligand-induced dimerization is then induced by a weak dimerization through the dimerization arms, and is immediately stabilized by strong dimerization of the kinase domains. The chance that (non-inhibited) kinase domains can also form dimers when the receptor is exhibiting the tethered conformation is only high enough when receptors are present in very high concentrations (pre-clustering). The pre-cluster arrangement does not allow the kinase to become active, due to an auto-inhibitory effect of the extracellular tethered conformation $^{78,79}$. We cannot exclude the possibility that the monomeric state coincides with a small population of weak dimers (e.g. a side-to-side arrangement), but these dimers have to be very transient in that case, unless stabilized in that arrangement by other means (e.g. by mAb 806). Furthermore, weak dimers could also be stabilized through artificial factors, such as nonspecific adhesion between fluorescent labels. Since stable dimerization is indeed only possible for receptors with a non-tethered extracellular conformation and an active kinase, the positive cooperativity of 
these two mechanisms explains the complex and subtle dimerization and activity conditions of ErbB receptors in a variety of receptor conditions.

We postulate the following model for the influence of cetuximab on EGFR. Upon cetuximab binding, the ligand binding site is occupied, and the receptor changes its conformation from the tethered to an alternative conformation. This conformation is different from the conformation induced by kinase inhibition, and allows for stable dimerization. Like the pre-dimerization conformation it lacks the ability for the kinase domains to become active and phosphorylate its partner, hence its inhibitory effects. However, we cannot exclude that the dimer formed is due to crosslinking caused by the bivalency of cetuximab, although this is unlikely due to the relatively inflexible way it binds to the receptor. This could potentially be verified by incubating the receptors with only the Fab fragment of the antibody.

The intriguing role of ErbB3 receptor in the ErbB signalling regulatory system is relevant for future drug discovery or prognosis of (combined) drug effectiveness. Here we showed that the dynamics of this receptor on the plasma membrane is influenced by binding of cetuximab to EGFR and again influenced by subsequent addition of EGF in a cell line benefitting from combined (EGFR and ErbB3) drugs treatment. Even when the cetuximab bound EGFRs do not directly bind ErbB3 receptors, the increase in diffusion speed of ErbB3 receptors triggers many questions. For example, how can an antibody targeting one receptor lead to different dynamics of another receptor? It would be interesting to determine whether cetuximab also influences the normal ErbB3 pathway, and if subsequent addition of its ligand HRG leads to more or a faster signalling response.

The study of altered diffusion speeds and the related dimerization using single-moleculetracking and analysis as presented here is also applicable to other receptor proteins to study dimerization under different receptor conditions.

\subsection{Conclusion}

Looking forward in anticancer therapy, there is a high need to find immunotherapy markers: ways to predict a patient's response to an immunotherapy or to show whether a given treatment is working ${ }^{80}$. As recently suggested, in vitro assays are a simple means for assessing the drug sensitivity of a patient's cancer ${ }^{81}$. The initial signalling and feed-back system defined by the molecular state and spatiotemporal distribution of ErbB receptors in the tumour cells might be representative for the response to distinct receptor antagonists. Also within the context of cultured cell lines there remain a lot of spatiotemporal and conformational questions to be answered. Such studies are relevant in the understanding of the way therapeutic antibodies might work on a monomeric or inactive population of receptors, i.e. by halting one or more of the steps involved in the ligand-induced dimer formation leading to activation of the kinase domain ${ }^{75}$.

In conclusion, we characterized the diffusion based mobility of EGFR under various receptor conditions by obtaining the two-populations diffusion parameters. We showed that certain spatiotemporal characteristics of EGFR are clearly distinct under different agonistic and antagonistic conditions, such as the diffusion speed, but not the organization of transiently immobile receptors in the plasma membrane. We reported on resting and liganded EGFR in MCF7 and H441 carcinoma cell lines, and showed a direct relation between dimerization and the diffusion coefficient of the fast population. Therapeutic antibodies and tyrosine kinase inhibitors alter the normal dimerization process of EGFR. Upon inhibition of the ligand-binding capability of EGFR by incubation with cetuximab, EGFR seems to form alternative dimers. We have found indications that ErbB3 is involved in this process, since it seems to get recruited from a preclustered state to a more active state, or may actually be the dimerization partner of the alternative EGFR dimer. The entanglement of ErbB3 receptors with the inhibition of EGFR needs further clarification. Furthermore we found that kinase inhibition by both erlotinib and lapatinib keeps 
the EGF receptor in a fast diffusing state which is independent of ligand addition. This is an indication that an active kinase is needed for stable dimerization. More detailed studies on the homo- and hetero-dimerization state of kinase inhibited receptors are however required to explain the conflicting results from immunoblotting and biophysical techniques. The final goal will be to find the spatiotemporal design principles of the complete ErbB system to cell decision-making, and how to manipulate this to get the system towards a healthy outcome.

\section{Acknowledgment}

We thank Jenny Ibach for providing SNAP-ErbB1 and SNAP-ErbB3 plasmid DNA. We thank Yvonne Kraan for culturing the cells.

\subsection{References}

1. Cebecauer, M., Spitaler, M., Sergé, A. \& Magee, A. I. Signalling complexes and clusters: functional advantages and methodological hurdles. J. Cell Sci. 123, 309-20 (2010).

2. Citri, A. \& Yarden, Y. EGF-ERBB signalling: towards the systems level. Nat. Rev. Mol. Cell Biol. 7, 505-16 (2006).

3. Yarden, Y. \& Pines, G. The ERBB network: at last, cancer therapy meets systems biology. Nat. Rev. Cancer 12, 553-63 (2012).

4. Yarden, Y. \& Sliwkowski, M. X. Untangling the ErbB signalling network. Nat. Rev. Mol. Cell Biol. 2, 127-37 (2001).

5. Lemmon, M. a. Ligand-induced ErbB receptor dimerization. Exp. Cell Res. 315, 638-48 (2009).

6. Lemmon, M. A. \& Schlessinger, J. Cell signaling by receptor tyrosine kinases. Cell 141, 1117-34 (2010).

7. Dengjel, J., Akimov, V., Blagoev, B. \& Andersen, J S. Signal transduction by growth factor receptors: signaling in an instant. Cell Cycle 6, 2913-6 (2007).

8. Avraham, R. \& Yarden, Y. Feedback regulation of EGFR signalling: decision making by early and delayed loops. Nat. Rev. Mol. Cell Biol. 12, 104-117 (2011).

9. Clayton, A. H. A., Tavarnesi, M. L. \& Johns, T. G. Unligated epidermal growth factor receptor forms higher order oligomers within microclusters on A431 cells that are sensitive to tyrosine kinase inhibitor binding. Biochemistry 46, 4589-97 (2007).

10. Hofman, E. G. et al. Ligand-induced epidermal growth factor receptor (EGFR) oligomerization is kinase-dependent and enhances internalization. $J$. Biol. Chem. 285, 39481-39489 (2010).

11. Kozer, N. et al. Differential and synergistic effects of epidermal growth factor receptor antibodies on unliganded ErbB dimers and oligomers. Biochemistry 50, 3581-90 (2011).

12. Szabó, Á., SzöllŐsi, J. \& Nagy, P. Coclustering of ErbB1 and ErbB2 Revealed by FRET-Sensitized Acceptor Bleaching. Biophys. J. 99, 105-114 (2010).

13. Keating, E., Nohe, A. \& Petersen, N. O. Studies of distribution, location and dynamic properties of EGFR on the cell surface measured by image correlation spectroscopy. Eur. Biophys. J. 37, 469-81 (2008)

14. Petersen, N. O., Höddelius, P. L., Wiseman, P. W., Seger, O. \& Magnusson, K. E. Quantitation of membrane receptor distributions by image correlation spectroscopy: concept and application. Biophys. J. 65, 1135-46 (1993).

15. Nagy, P., Claus, J., Jovin, T. M. \& Arndt-Jovin, D. J. Distribution of resting and ligand-bound ErbB1 and ErbB2 receptor tyrosine kinases in living cells using number and brightness analysis. Proc. Natl. Acad. Sci. U. S. A. 107, 16524-9 (2010).

16. Digman, M. a, Dalal, R., Horwitz, A. F. \& Gratton, E. Mapping the number of molecules and brightness in the laser scanning microscope. Biophys. J. 94, 232032 (2008).

17. Saxton, M. J. \& Jacobson, K. Single-particle tracking: applications to membrane dynamics. Annu. Rev. Biophys. Biomol. Struct. 26, 373-99 (1997).

18. Low-Nam, S. T. et al. ErbB1 dimerization is promoted by domain co-confinement and stabilized by ligand binding. Nat. Struct. Mol. Biol. 18, 1244-1249 (2011).

19. Engelman, J. A. \& Settleman, J. Acquired resistance to tyrosine kinase inhibitors during cancer therapy. Curr. Opin. Genet. Dev. 18, 73-9 (2008).

20. Ledford, H. Immunotherapy's cancer remit widens. Nature 497, 544 (2013).

21. Galon, J. et al. Type, density, and location of immune cells within human colorectal tumours predict clinical outcome. Science 313, 1960-4 (2006).

22. Schaefer, G. et al. A two-in-one antibody against HER3 and EGFR has superior inhibitory activity compared with monospecific antibodies. Cancer Cell 20, 472-86 (2011).

23. Landgraf, R. HER2 therapy. HER2 (ERBB2): functional diversity from structurally conserved building blocks. Breast Cancer Res. 9, 202 (2007).

24. Shi, F., Telesco, S. E., Liu, Y., Radhakrishnan, R. \& Lemmon, M. A. ErbB3/HER3 intracellular domain is competent to bind ATP and catalyze autophosphorylation. Proc. Natl. Acad. Sci. U. S. A. 107, 7692-7 (2010).

25. Taylor, S. S. \& Kornev, A. P. Yet another "active" pseudokinase, Erb3. Proc. Natl. Acad. Sci. U. S. A. 107, 8047-8 (2010).

26. Jura, N. et al. Mechanism for activation of the EGF receptor catalytic domain by the juxtamembrane segment. Cell 137, 1293-307 (2009).

27. Zhu, H.-J., laria, J., Orchard, S., Walker, F. \& Burgess, A. W. Epidermal Growth Factor Receptor: Association of Extracellular Domain Negatively Regulates Intracellular Kinase Activation in the 
Absence of Ligand. Growth Factors 21, 15-30 (2003).

28. Pollack, V. A. et al. Inhibition of Epidermal Growth Factor Receptor-Associated Tyrosine Phosphorylation in Human Carcinomas with CP-358,774: Dynamics of Receptor Inhibition In Situ and Antitumour Effects in Athymic Mice. J. Pharmacol. Exp. Ther. 291, 739-748 (1999).

29. Rusnak, D. W. et al. The effects of the novel, reversible epidermal growth factor receptor/ErbB-2 tyrosine kinase inhibitor, GW2016, on the growth of human normal and tumour-derived cell lines in vitro and in vivo. Mol. Cancer Ther. 1, 85-94 (2001).

30. Li, S. et al. Structural basis for inhibition of the epidermal growth factor receptor by cetuximab. Cancer Cell 7, 301-11 (2005)

31. Bellot, F. et al. High-affinity epidermal growth factor binding is specifically reduced by a monoclonal antibody, and appears necessary for early responses. J. Cell Biol. 110, 491-502 (1990).

32. Ferguson, K. M. Structure-based view of epidermal growth factor receptor regulation. Annu. Rev. Biophys. 37, 353-73 (2008).

33. Arteaga, C. L., Ramsey, T. T., Shawver, L. K. \& Guyer, C. A. Unliganded epidermal growth factor receptor dimerization induced by direct interaction of quinazolines with the ATP binding site. J. Biol. Chem. 272, 23247-54 (1997)

34. Lichtner, R. B., Menrad, A., Sommer, A., Klar, U. \& Schneider, M. R. Signaling-inactive epidermal growth factor receptor/ligand complexes in intact carcinoma cells by quinazoline tyrosine kinase inhibitors. Cancer Res. 61, 5790-5 (2001).

35. Gan, H. K. et al. The epidermal growth factor receptor (EGFR) tyrosine kinase inhibitor AG1478 increases the formation of inactive untethered EGFR dimers. Implications for combination therapy with monoclonal antibody 806. J. Biol. Chem. 282, 2840 50 (2007).

36. Wang, Q., Zhu, F. \& Wang, Z. Identification of EGF receptor C-terminal sequences 1005-1017 and dileucine motif 1010LL1011 as essential in EGF receptor endocytosis. Exp. Cell Res. 313, 3349-63 (2007).

37. Bose, R. et al. Activating HER2 mutations in HER2 gene amplification negative breast cancer. Cancer Discov. 3, 224-37 (2013).

38. Clayton, A. H. a et al. Ligand-induced dimer-tetramer transition during the activation of the cell surface epidermal growth factor receptor-A multidimensional microscopy analysis. J. Biol. Chem. 280, 30392-9 (2005).

39. Baselga, J. \& Swain, S. M. Novel anticancer targets: revisiting ERBB2 and discovering ERBB3. Nat. Rev. Cancer 9, 463-75 (2009).

40. Jiang, N., Saba, N. F. \& Chen, Z. G. Advances in Targeting HER3 as an Anticancer Therapy. Chemother. Res. Pract. 2012, 817304 (2012).

41. Kusumi, A. et al. Paradigm shift of the plasma membrane concept from the two-dimensional continuum fluid to the partitioned fluid: high-speed single-molecule tracking of membrane molecules. Annu. Rev. Biophys. Biomol. Struct. 34, 351-78 (2005).

42. Brameshuber, M. \& Schütz, G. J. in Springer Series on Fluorescence 1-37 (Springer Berlin Heidelberg, 2012). doi:10.1007/4243_2011_38

43. Brauchle, C., Lamb, D. C. \& Michaelis, J. Single Particle Tracking and Single Molecule Energy Transfer. (WILEY-VCH Verlag GmbH).
44. Cambi, A. \& Lidke, D. S. Nanoscale membrane organization: where biochemistry meets advanced microscopy. ACS Chem. Biol. 7, 139-49 (2012).

45. Gambin, Y. et al. Lateral mobility of proteins in liquid membranes revisited. Proc. Natl. Acad. Sci. U. S. A. 103, 2098-102 (2006).

46. Cutler, P. J. et al. Multi-color quantum dot tracking using a high-speed hyperspectral line-scanning microscope. PLoS One 8, e64320 (2013).

47. Smith, C. S., Joseph, N., Rieger, B. \& Lidke, K. A. Fast, single-molecule localization that achieves theoretically minimum uncertainty. Nat. Methods 7 , 373-5 (2010)

48. Lidke, D. S., Low-Nam, S. T., Cutler, P. J. \& Lidke, K. A. Determining FcERI diffusional dynamics via single quantum dot tracking. Methods Mol. Biol. 748 , 121-32 (2011)

49. Schütz, G. J., Schindler, H. \& Schmidt, T. Singlemolecule microscopy on model membranes reveals anomalous diffusion. Biophys. J. 73, 1073-80 (1997)

50. Saxton, M. J. Lateral diffusion in an archipelago. Single-particle diffusion. Biophys. J. 64, 1766-80 (1993).

51. Elliott, L. C. C., Barhoum, M., Harris, J. M. \& Bohn, P. W. Trajectory analysis of single molecules exhibiting non-brownian motion. Phys. Chem. Chem. Phys. 13, 4326-34 (2011).

52. Keppler, A. et al. A general method for the covalent labeling of fusion proteins with small molecules in vivo. Nat. Biotechnol. 21, 86-9 (2003).

53. Keppler, A., Pick, H., Arrivoli, C., Vogel, H. \& Johnsson, K. Labeling of fusion proteins with synthetic fluorophores in live cells. Proc. Natt. Acad. Sci. U. S. A. 101, 9955-9 (2004).

54. Gautier, A. et al. An engineered protein tag for multiprotein labeling in living cells. Chem. Biol. 15, 128-36 (2008).

55. Sun, X. et al. Development of SNAP-tag fluorogenic probes for wash-free fluorescence imaging. Chembiochem 12, 2217-26 (2011).

56. Corrêa, I. R. et al. Substrates for improved live-cell fluorescence labeling of SNAP-tag. Curr. Pharm. Des. 19, 5414-20 (2013)

57. Calebiro, D. et al. Single-molecule analysis of fluorescently labeled G-protein-coupled receptors reveals complexes with distinct dynamics and organization. Proc Natl Acad Sci U S A 110, 743-748 (2013).

58. Stortelers, C. The role of EGF-like growth factors in selective ErbB receptor dimerisation, $\mathrm{PhD}$ thesis, Radboud Universiteit Nijmegen. (2003).

59. De Keijzer, S. et al. A spatially restricted increase in receptor mobility is involved in directional sensing during Dictyostelium discoideum chemotaxis. J. Cell Sci. 121, 1750-7 (2008).

60. Kapanidis, A. N. \& Strick, T. Biology, one molecule at a time. Trends Biochem. Sci. 34, 234-43 (2009).

61. Verveer, P. J. Quantitative Imaging of Lateral ErbB1 Receptor Signal Propagation in the Plasma Membrane. Science 290, 1567-1570 (2000).

62. Sako, Y., Minoghchi, S. \& Yanagida, T. Singlemolecule imaging of EGFR signalling on the surface of living cells. Nat. Cell Biol. 2, 168-72 (2000).

63. Chung, I. et al. Spatial control of EGF receptor activation by reversible dimerization on living cells. Nature 464, 783-7 (2010).

64. Wieser, S., Axmann, M. \& Schütz, G. J. Versatile analysis of single-molecule tracking data by comprehensive testing against Monte Carlo simulations. Biophys. J. 95, 5988-6001 (2008). 
65. Sigismund, S. et al. Clathrin-mediated internalization is essential for sustained EGFR signaling but dispensable for degradation. Dev. Cell 15, 209-19 (2008).

66. Doody, J. F. et al. Inhibitory activity of cetuximab on epidermal growth factor receptor mutations in non small cell lung cancers. Mol. Cancer Ther. 6, 264251 (2007).

67. Walsh, N. et al. EGFR and HER2 inhibition in pancreatic cancer. Invest. New Drugs 31, 558-66 (2013).

68. Kong, A. et al. HER2 oncogenic function escapes EGFR tyrosine kinase inhibitors via activation of alternative HER receptors in breast cancer cells. PLoS One 3, e2881 (2008).

69. Kani, K., Warren, C. M., Kaddis, C. S., Loo, J. A. \& Landgraf, R. Oligomers of ERBB3 have two distinct interfaces that differ in their sensitivity to disruption by heregulin. J. Biol. Chem. 280, 8238-47 (2005).

70. Lidke, D. S. et al. Quantum dot ligands provide new insights into erbB/HER receptor-mediated signal transduction. Nat. Biotechnol. 22, 198-203 (2004).

71. Arndt-Jovin, D. Quantum dots shed light on processes in living cells. SPIE Newsroom 2-4 (2006). doi: $10.1117 / 2.1200605 .0228$

72. Montiel, D., Cang, H. \& Yang, H. Quantitative characterization of changes in dynamical behavior for single-particle tracking studies. J. Phys. Chem. B 110, 19763-70 (2006).

73. Pryor, M. M. et al. Dynamic Transition States of ErbB1 Phosphorylation Predicted by Spatial
Stochastic Modeling. Biophys. J. 105, 1533-43 (2013).

74. Offterdinger, M., Georget, V., Girod, A. \& Bastiaens, P. I. H. Imaging phosphorylation dynamics of the epidermal growth factor receptor. J. Biol. Chem. 279, 36972-81 (2004).

75. Schmitz, K. R. \& Ferguson, K. M. Interaction of antibodies with ErbB receptor extracellular regions. Exp. Cell Res. 315, 659-70 (2009).

76. Ziomkiewicz, I. et al. Dynamic conformational transitions of the EGF receptor in living mammalian cells determined by FRET and fluorescence lifetime imaging microscopy. Cytometry. A 83, 794-805 (2013).

77. Johns, T. G. et al. Identification of the epitope for the epidermal growth factor receptor-specific monoclonal antibody 806 reveals that it preferentially recognizes an untethered form of the receptor. J. Biol. Chem. 279, 30375-84 (2004).

78. Endres, N. F. et al. Conformational coupling across the plasma membrane in activation of the EGF receptor. Cell 152, 543-56 (2013).

79. Arkhipov, A. et al. Architecture and membrane interactions of the EGF receptor. Cell 152, 557-69 (2013).

80. Ledford, H. Sizing up a slow assault on cancer. Nature 496, 14-15 (2013).

81. Editorial. Dishing out cancer treatment. Nat. Biotechnol. 31, 85-85 (2013). 



\section{Supporting Information}

\section{The diffusion of single EGF receptors relates to their dimerization state and is altered by antagonists}

Peter J. Bosch ${ }^{1}$

Johannes S. Kanger ${ }^{1}$ Vinod Subramaniam ${ }^{1,2}$

1. Nanobiophysics, MESA+ Institute for Nanotechnology and MIRA Institute for Biomedical Technology and Technical Medicine, University of Twente, PO Box 217, 7500AE Enschede, The Netherlands

2. Present address: FOM Institute AMOLF, Science Park 104, 1098 XG Amsterdam, The Netherlands

This work entails collaborations with

Anton G.T. Terwisscha van Scheltinga

K.J.D. Kol

Thijs Oude Munnink

Steven de Jong

Elisabeth G. E. de Vries

affiliated at 


\section{Supporting figures}

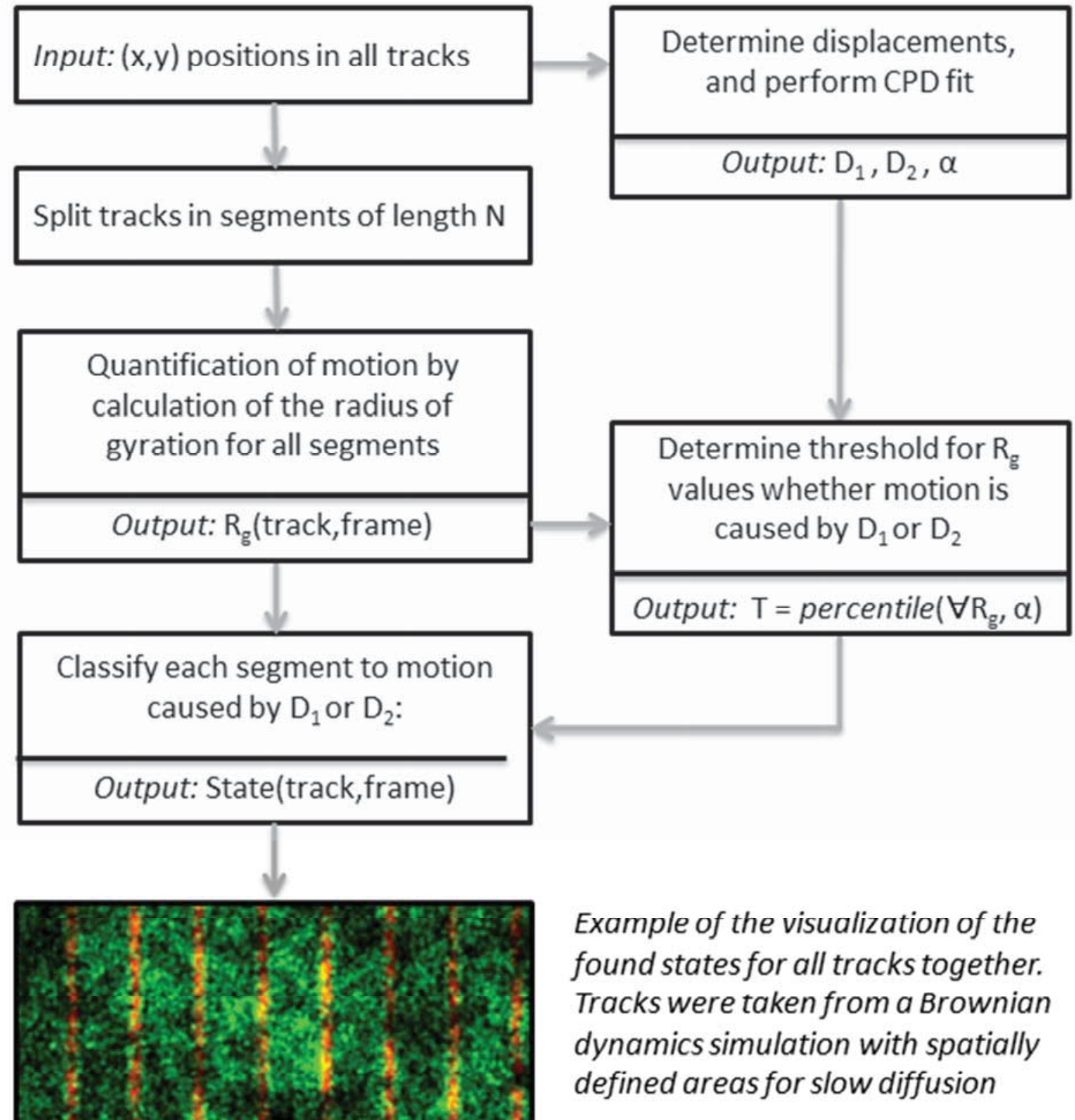

FIGURE S1 Scheme of the methodology followed to classify the motion at each position in a track as fast or slow diffusion. After trajectories of molecules are found with a tracking algorithm, we determine the diffusion constants $\left(D_{1}\right.$ and $\left.D_{2}\right)$ and the fraction $(\alpha)$ of the fast state from all displacements using a CPD fit. The track is divided in segments of a certain window length $(N)$, and the radius of gyration $\left(R_{g}\right)$ is determined for all segments. The threshold $(T)$ for classification of the motion within a segment to a diffusion with diffusion constant $D_{1}$ or $D_{2}$ is determined from all $R_{g}$ values and the fraction $\alpha$. The center position of the segment is classified as slow diffusion when $R_{g}$ is smaller than the threshold $T$, and as fast diffusion otherwise. The classified states are visualized in a super-resolution image, where the positions in the tracks are visualized with a dot. The color of the dot depends on the found state at that position: green for the fast state, and red for the slow state. The visualization example shows that simulated tracks, where two states with different diffusion speeds $\left(D_{1}=0.17 \mu \mathrm{m}^{2} / \mathrm{s}, D_{2}=0.015 \mu \mathrm{m}^{2} / \mathrm{s}\right)$ are spatially separated, are indeed suitably classified to clearly identify the regions of slow diffusion. 

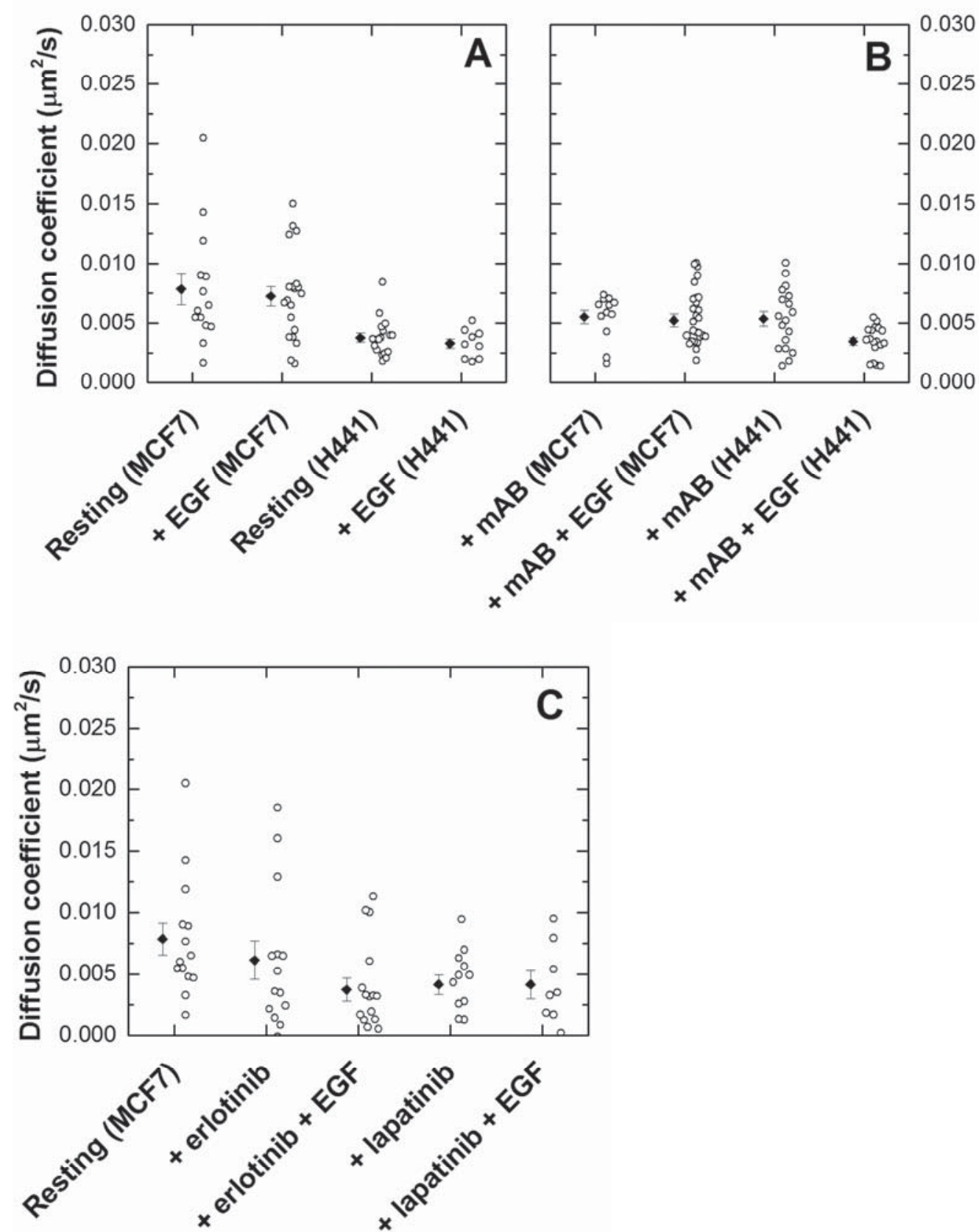

FIGURE S2 Diffusion coefficient of the slow fraction of EGFR under different conditions. The open circles are the diffusion coefficients found in the microscopy recordings for different cells measured in one condition. The closed circle shows the mean and the standard error of all recordings. (A) Diffusion coefficient (slow fraction) of EGFR in MCF7 and H441 cells in the presence of ligand. (B) Diffusion coefficient (slow fraction) of EGFR in MCF7 and H441 cells in the presence of cetuximab. (C) Diffusion coefficient (slow fraction) of EGFR in MCF7 cells in the presence of kinase inhibitors. 

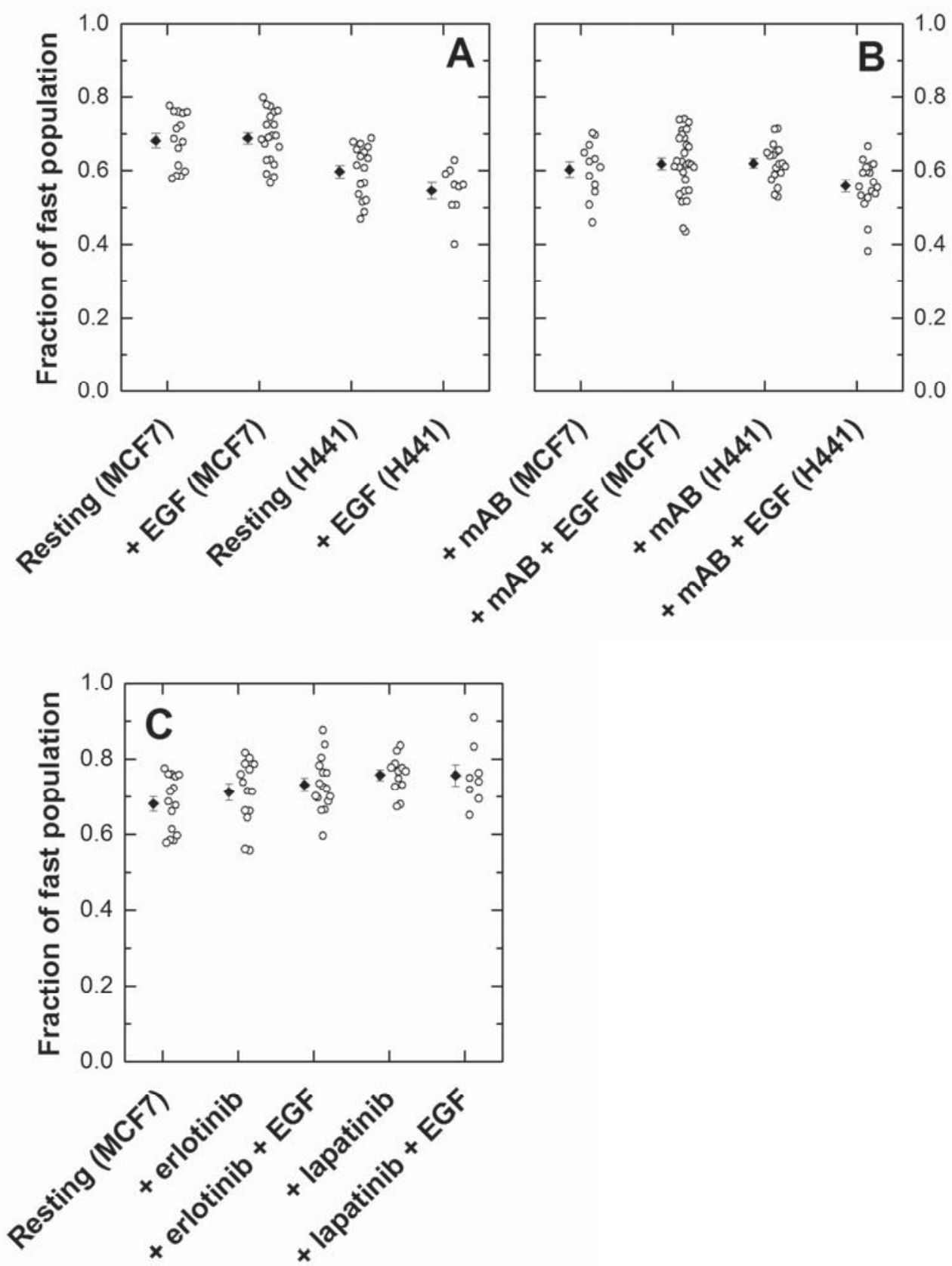

FIGURE S3 Fraction size of the fast population of EGFR under different conditions. The open circles are the diffusion coefficients found in the microscopy recordings for different cells measured in one condition. The closed circle shows the mean and the standard error of all recordings. (A) Fraction size of the fast population of EGFR in MCF7 and H441 cells in the presence of ligand. (B) Fraction size of the fast population of EGFR in MCF7 and H441 cells in the presence of cetuximab. (C) Fraction size of the fast population of EGFR in the presence of kinase inhibitors. 
A

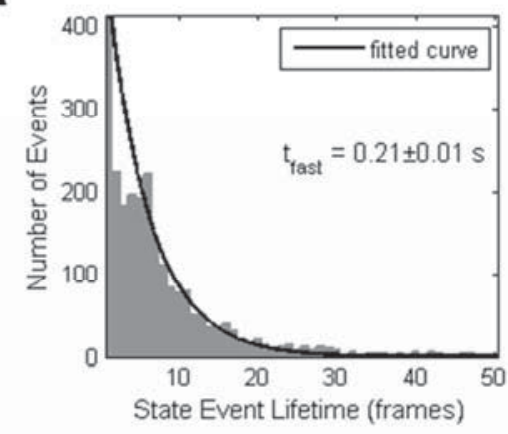

C

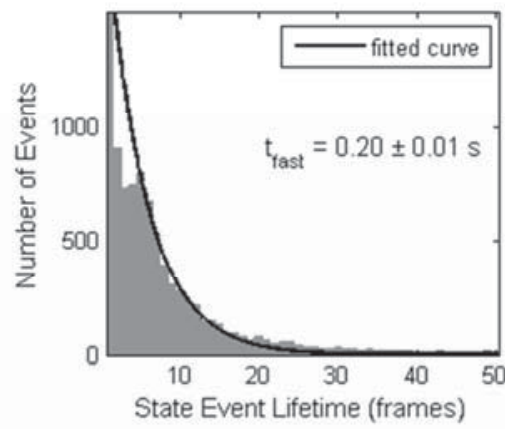

E

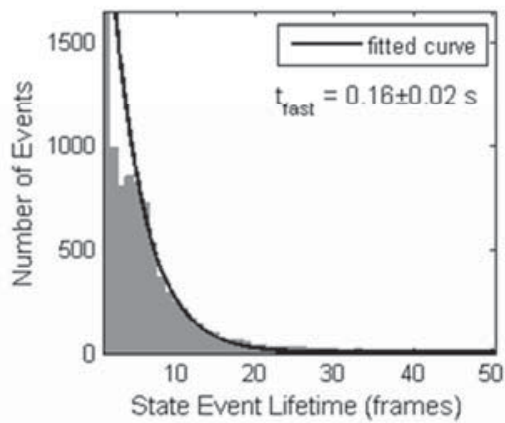

G

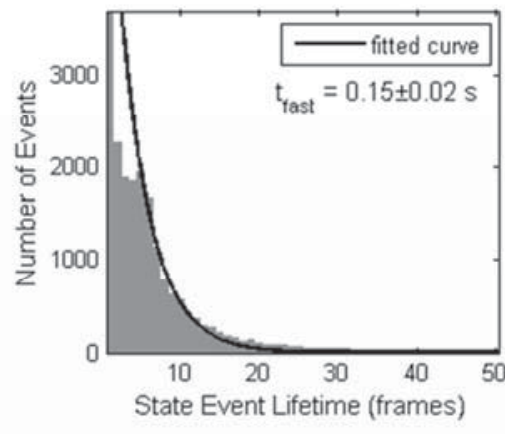

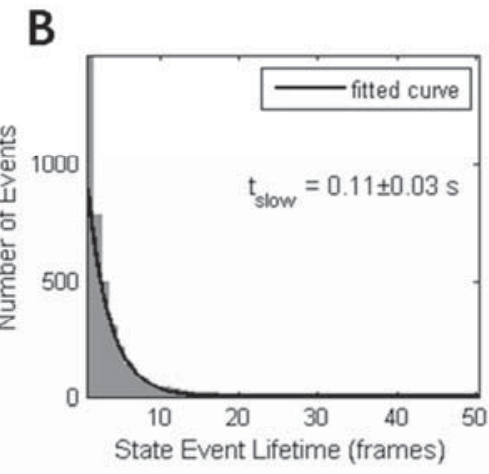

D

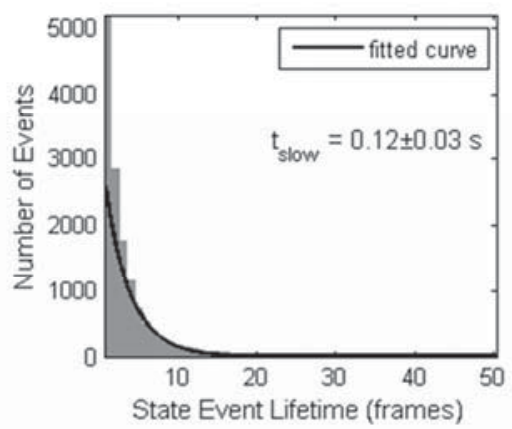

F

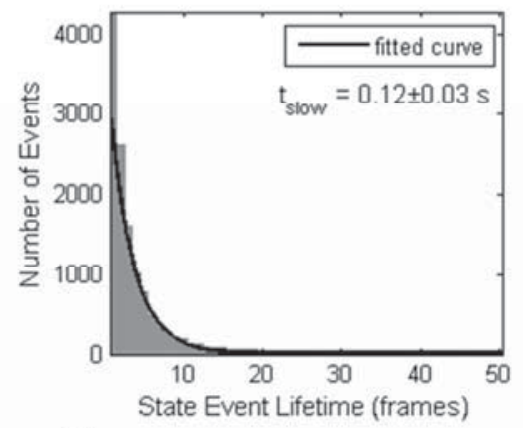

H

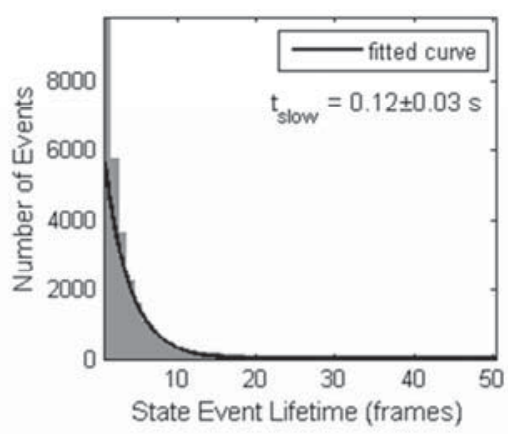

FIGURE S4 Histograms of EGF receptor state lifetimes for the fast state (left) and the slow state (right) under different receptor conditions in MCF7 cells. The receptor conditions tested were unliganded $(A, B)$, liganded $(C, D)$, with cetuximab $(E, F)$, and cetuximab with subsequent ligand addition $(G, H)$. The lifetimes are measured in frames (video was recorded at $25 \mathrm{fps}$ ), and were determined by gyration based diffusion state classification. The characteristic lifetime is determined from an exponential fit of state durations longer than 5 frames, since the use of a segment length of 7 frames does not correctly resolve shorter lifetimes. The state duration will be underestimated because of random incorrect state classification. 


\section{Supporting tables}

TABLE S1 Diffusion coefficient (fast fraction) statistics of EGFR in MCF7 and H441 cells and ErbB3 in FaDu cells under the different conditions tested. The sample size $\mathrm{N}$ is the number of recordings (with a maximum of two recordings per cell) measured for each condition.

\begin{tabular}{|ll|lll|}
\hline EGFR in MCF7 cells & N total & Mean & $\begin{array}{l}\text { SE of } \\
\text { mean }\end{array}$ & $\begin{array}{l}\text { Standard } \\
\text { Deviation }\end{array}$ \\
\hline Resting & 14 & 0.148 & 0.006 & 0.024 \\
\hline + EGF & 20 & 0.112 & 0.006 & 0.025 \\
\hline + cetuximab & 12 & 0.092 & 0.005 & 0.016 \\
\hline + cetuximab + EGF & 28 & 0.089 & 0.005 & 0.029 \\
\hline + erlotinib & 14 & 0.157 & 0.009 & 0.032 \\
\hline + erlotinib + EGF & 16 & 0.163 & 0.007 & 0.029 \\
\hline + lapatinib & 12 & 0.161 & 0.006 & 0.022 \\
\hline + lapatinib + EGF & 8 & 0.163 & 0.022 & 0.062 \\
\hline
\end{tabular}

\begin{tabular}{|l|c|l|ll|}
\hline EGFR in H441 cells & N total & Mean & $\begin{array}{l}\text { SE of } \\
\text { mean }\end{array}$ & $\begin{array}{l}\text { Standard } \\
\text { Deviation }\end{array}$ \\
\hline Resting & 17 & 0.132 & 0.006 & 0.023 \\
\hline + EGF & 9 & 0.114 & 0.007 & 0.022 \\
\hline + cetuximab & 18 & 0.117 & 0.004 & 0.016 \\
\hline + cetuximab + EGF & 18 & 0.078 & 0.005 & 0.020 \\
\hline
\end{tabular}

\begin{tabular}{|lcc|c|c|}
\hline ErbB3 in Fadu cells & N total & Mean & $\begin{array}{l}\text { SE of } \\
\text { mean }\end{array}$ & $\begin{array}{l}\text { Standard } \\
\text { Deviation }\end{array}$ \\
\hline Resting & 18 & 0.081 & 0.005 & 0.020 \\
\hline + EGF & 22 & 0.087 & 0.007 & 0.033 \\
\hline + cetuximab & 8 & 0.130 & 0.010 & 0.029 \\
\hline + cetuximab + EGF & 20 & 0.107 & 0.009 & 0.040 \\
\hline
\end{tabular}




\section{Run-file for HMM comovement analysis}

\section{$(\ldots)$}

HMM_PP = HMM_PreProcessStates([hmmdir 'savedir'],'All', [hmmdir 'savedirl' 'HMM_folder.HMMPP'],100,400, 0.5, 3, 10, 0.001, 5, .200);

\% \{'HMM DataFile Directory','Data Conditions','OutPutFile', 'Max Dimer Separation (nm)','Domain Size (nm)','Analysis Threshold L (microns)','Min Overlapping Valid Frames', 'Min Traj Length (frames)','Duty Cycle','Min Overlapping Time (Frames)','Close Approach Threshold (microns)'\}

\% NOTE(!), I think 'Min Traj Length (frames' and 'Min Overlapping Time (Frames)' are confused here!!

$\%$ NOTE (!!): to the dimer and domain size all sigmas (loc errors) will be added as well by the function, but to appThresh it is -not-!

$\% \mathrm{~L}=0.5$; \%micron. Analysis is restricted to frames where separation is less than $\mathrm{L}$.

$\%$ NOTE (!!!): should be large enough so that the p-chance for a non-free state is calculated (and small), such that not a non-free state is interpolated!!!

$\%$ MinOverlap=3; \%frames. Minimum number of overlapping valid frames of two tracks for inclusion in analysis

\% MinPath=10; \%frames. \%rule out tracks that don't overlap for enough frames \% Tracks must have valid points separated by this value or larger or are excluded

\% DC=.001; \%duty cycle. Tracks must have a (valid frames)/(track length) ratio greater than DC

$\%$ minOLT $=5$; \%frames. Minimum number of overlapping frames (valid or invalid) of two tracks for inclusion in analysis

$\%$ appThresh $=.2 ; \%$ micron Tracks must get this close at least once to be included (see as quick throw away setting) 



\section{Chapter 6}

Single-molecule tracking of plasma membrane proteins combined with local actuation using ligand functionalised AFM tips 


\begin{abstract}
We have demonstrated that functionalized AFM tips can be combined with single-molecule fluorescence microscopy, such that single receptors on the plasma membrane can be tracked whilst locally actuating a subpopulation of receptors. For that, we milled the bottom part of an AFM tip using a focused ion beam to obtain a tip-cell contact area of approximately $2 \times 2 \mu \mathrm{m}$ with the idea that several ligand molecules are presented to the receptors of the cell. Cysteine mutated EGF was then attached to an amino coated milled AFM tip. To confirm the specificity of the functionalization chemistry, we devised an immunofluorescence assay. Immunostaining of an EGF functionalized AFM tip indicated that EGF indeed attaches to the pyramid shaped AFM tip. A functionalized AFM tip was then used to locally induce an interaction with EGF receptors in live cells. Simultaneously, hetero-dimerization of EGFR with the ErbB2 receptor, its preferred heterodimerization partner, was monitored by observing the stop of motion of individual ErbB2 receptors with single molecule tracking microscopy. This immobilization was specific for the tipcell contact area. A negative control is still required to confirm the specificity of the interaction, although it is unlikely that the AFM tip can nonspecifically interact with the membrane due to the polyethylene glycol linkers conjugated to the tip.
\end{abstract}




\subsection{Introduction}

The study of individual single molecules has allowed us to better understand the design principles and appreciate the molecular heterogeneities of many biological processes. The methods to visualize and localize single molecules in biology has developed to become a standard microscopy tool in the field with super-resolution approaches such as Stochastic Optical Reconstruction Microscopy (STORM) and Stimulated Emission Depletion (STED) microscopy now being commercially available and widely used. In contrast, the manipulation of single molecules is still an $\operatorname{art}^{1-4}$, which opens the possibility to study local chemical and biophysical processes. Most chemical interactions with cells are typically investigated in bulk with soluble ligands. However, a locally applied stimulus can provide key insights to reveal elusive details on the signalling design principles of the cell. For example, it was shown using ligand functionalized micron-sized beads that plasma membrane receptors may amplify signals by a lateral signal propagation starting from the site of ligand-induced activation ${ }^{5}$. A hint ${ }^{6}$ lead to the explanation of this propagation; the fast diffusion of hydrogen peroxide $\left(\mathrm{H}_{2} \mathrm{O}_{2}\right)$ released upon activation of the receptor activates the tyrosine kinases of inactive receptors ${ }^{7}$. The question remains why hydrogen peroxide, which has a short lifetime, can influence receptors over such a wide range in the cell, and how this mechanism can be selective for EGFR while numerous other phosphorylation-dependent signals exist ${ }^{8}$. A similar local activation can be achieved with a ligand functionalized AFM tip, which has been used extensively in AFM based force spectroscopy to probe the interaction forces between two biomolecules $^{9-14}$. An atomic force microscopy (AFM) based approach has the advantage over a bead that this activation probe can be precisely guided to a particular spot at the cell membrane and allows control over the duration of interaction as the probe can be retracted at any chosen time. Certainly the activity of the biomolecules that are incubated on the AFM tip will result in a cellular response in live cells, such that the AFM tip becomes a highly controllable actuation tool. We aimed to functionalize AFM tips to locally position ligands to a small part of the cell surface in a force-guided manner (Fig. 6.1), and to follow the molecular response of the cell using fluorescence microscopy.

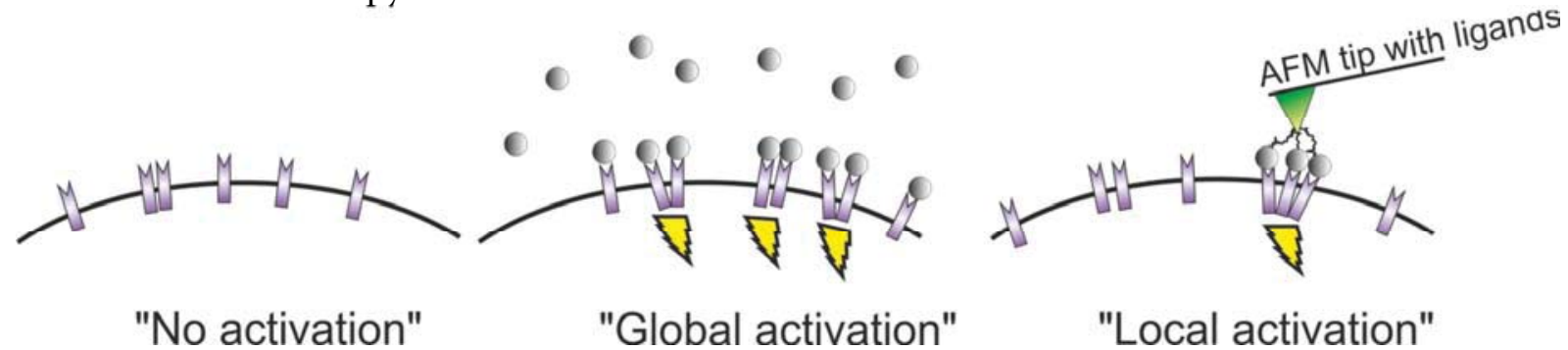

FIGURE 6.1 Schematic representation to appreciate the difference between a global and local activation of cellular proteins. In a common experiment receptor are first starved (left) and subsequently soluble ligand (spheres) is added to the cell medium, resulting in a global activation of plasma membrane bound receptors (middle). A ligand functionalised AFM tip can induce a local actuation of the receptors residing in a particular part of the membrane (right). The stimulus can also be removed by retracting the AFM tip at any chosen time.

Several AFM based force spectroscopy experiments using antibodies on the AFM tip and receptors on the plasma membrane of live cells have been reported in the last years ${ }^{15-19}$. The incubation of an antibody to an AFM tip has become more or less a routine procedure ${ }^{20,21}$, which can also be used to probe receptors in live cells ${ }^{22}$. Also, cells have been attached to the AFM tip to study cell-cell adhesion with another cell on the surface ${ }^{23}$, and in another study, the surface was functionalized with ligand to study the ALCAM - CD6 interaction ${ }^{24}$. For smaller biomolecules however, experimental protocols are not well established and require custom optimization due to the lack of freely accessible binding targets outside the binding pocket, such as free amino or thiol 
groups in lysine and cysteine amino acid residues respectively. Only recently a receptor actuating ligand (MFZ2-12) has been attached on an AFM tips and used to probe serotonin transporter in live $\mathrm{CHO}-\mathrm{K} 1$ cells to determine the energy landscape of these molecules ${ }^{25}$. The authors of the last report did not monitor the molecular response of the cell in that study. Also, they explicitly mentioned that not all cell lines are suitable for live cell force spectroscopy due to large excursions of the membrane of cells during AFM imaging, such as for HEK293 cells. So far, monitoring the cellular response by optical microscopy after AFM based actuation with chemoattractants has only been based on white light microscopy (to the best of our knowledge). For example to observe the appearance of filopodia and bleb formation after applying a mechanical stimulus by untreated AFM tips ${ }^{26}$, and to monitor cytoskeletal remodelling after local actuation by AFM tips functionalised with HA molecules which target CD44 receptors ${ }^{10}$.

In this chapter we describe the methodological developments to attach epidermal growth factor (EGF) to the silicon material of AFM tips. For this we used flexible heterobifunctional polyethylene glycol (PEG) crosslinkers, which allows the protein to be flexible and present itself in all orientations to a recognizing protein. We tested the functionalization by force spectroscopy as described earlier ${ }^{20,21}$. Since the aldehyde-linker chemistry did not show satisfactory results, we used a cysteine mutant of EGF to realize the attachment of ligand to the tip. To avoid timeconsuming force spectroscopy experiments to confirm the attachment chemistry, we reasoned and confirmed that a single-molecule immunofluorescence assay is a suitable method to validate attachment. Furthermore we investigate other criteria for the AFM tip for combined use of AFM and single-molecule fluorescence microscopy. Finally, we show preliminary results of live cell experiments where we performed local actuation of EGF receptor (EGFR) and performed singlemolecule tracking on its preferred hetero-dimerization partner.

\subsection{Methods}

\subsubsection{Amino-functionalization of the AFM tips}

AFM tips were functionalized according to protocols described by others ${ }^{20,27}$. The tips were washed 3 times for 5 minutes in chloroform, washed 3 times for 5 minutes in ethanol, and dried with nitrogen. Thereafter, an (3-aminopropyl)triethoxysilane (APTES) monolayer was deposited on the tips by gas vapour deposition ${ }^{28}$. For this, the tips were put on an inert Teflon supporting surface in a 5 litre desiccator, which was then flooded with argon for 10 minutes to remove air and moisture. Two plastic cups (lids of Eppendorf tubes) were filled separately with $60 \mu$ distilled APTES (stored in argon environment) and $20 \mu \mathrm{l}$ triethylamine (stored in argon environment). The cups were placed in the desiccator, which was then flushed with argon for 1 minute. After two hours of incubation the cups were removed, and the desiccator was slowly flushed for 5 minutes with argon gas to allow the APTES to cure for at least 2 days (there should be almost zero humidity, but a tiny bit of water is required for the curing). The tips were then directly used for functionalization with a heterobifunctional PEG-linker and EGF.

\subsubsection{EGF incubation of the AFM tips}

For the force spectroscopy experiments we used an aldehyde-linker ${ }^{29}$ with EGF incubation performed at $\mathrm{pH} 5.5$ to specifically target the exposed amine group of the $\mathrm{N}$-terminus of $\mathrm{EGF}^{30}$. This $\mathrm{pH}$ reduces the chance that the $\varepsilon$-amino groups in the lysine amino acids of the protein in the binding pocket of EGF are targeted, which would block the protein functionality. The other end of the linker has an N-hydroxy succinimide (NHS) ester which is much more reactive than aldehyde. With careful timing and concentration, this side of the linker binds the amine functionalised surface first, such that the aldehyde group can subsequently bind an amine group of a protein, as shown in Fig. 6.2. Aldehyde-PEG-NHS linkers were a gift from A. Ebner (University of Linz, 
Institute of Biophysics, Austria). One portion of aldehyde-linker $(3.3 \mathrm{mg}$ ) was added to $0.5 \mathrm{~mL}$ chloroform $(6.6 \mathrm{mg} / \mathrm{mL})$, and vortexed until dissolved. The APTES coated tips were placed into a Teflon chamber filled with the linker solution, and 15-30 $\mu \mathrm{L}$ triethylamine was added to start the NHS reaction. This incubation allows the APTES amine groups to react with the NHS ester function, forming a stable amide bond. A glass lid was placed onto the Teflon chamber to prevent evaporation of the chloroform. After 2 hours the tips were washed with chloroform as described above and extensively dried. Recombinant human EGF (Pan Biotech, Germany) was dissolved in PBS and stored in aliquots of $200 \mu \mathrm{L}$ of $200 \mu \mathrm{g} / \mathrm{mL}$ concentration at $-80{ }^{\circ} \mathrm{C}$. One portion of EGF was mixed with $2 \mu \mathrm{l}$ of a $1 \mathrm{M}$ sodium cyanoborohydride water (with $10 \mathrm{mM} \mathrm{NaOH}$ ) solution, and added to the tips. The cyanoborohydride act as a mild reducing agent for the Schiff base (imine bond) formed from the aldehyde and the amino group of EGF. After 2 hours incubation, $50 \mathrm{mM}$ ethanolamine was added to react with the remaining aldehyde groups. After 10 minutes, the tips were washed with PBS, and stored at $4{ }^{\circ} \mathrm{C}$ for up to 2 days.

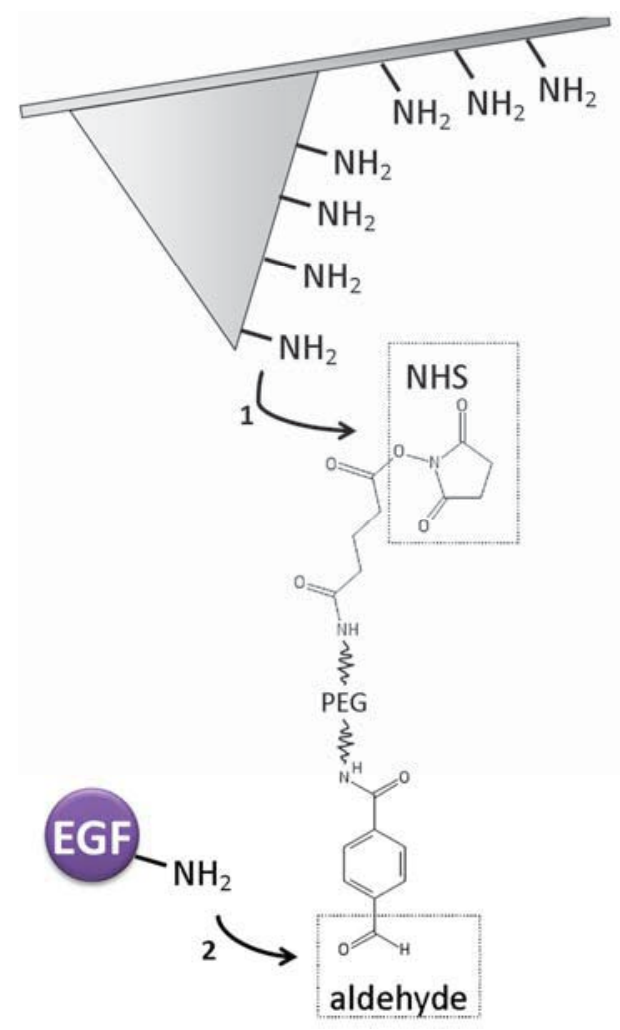

FIGURE 6.2 Schematic representation of the aldehydelinker based method for tip-protein attachment. The NHS group of the heterobifunctional linker bind the APTES coated AFM tip, whereas subsequently the aldehyde group binds amine groups on a protein such as the $\mathrm{N}$-terminus of EGF. The maleimide-linker (not shown) has a maleimide group instead of an aldehyde group, and binds to the thiol group in cysteine mutated EGF.

The immunofluorescence assay and live cell experiments used an optimized method, as a cysteine mutant of EGF (cys-EGF, a gift from M. Sonntag, Laboratory of Chemical Biology, TU Eindhoven, The Netherlands) had become available to us at that time. The incubation using a maleimide (MAL) group on the linker to bind the unprotected thiol in the cysteine of cys-EGF is faster than the aldehyde reaction, and is therefore less prone to hydration which reduces the number of EGF molecules binding to the linker. It is also better defined as there is only one available thiol in cys-EGF, and maleimide reacts relatively fast with this thiol. The maleimide linkers were obtained from Polypure (Oslo, Norway). Under an argon gas environment $2.5 \mathrm{mg}$ MAL-PEG-NHS linker was added to $2.5 \mathrm{~mL}$ chloroform $(1 \mathrm{mg} / \mathrm{mL})$, and vortexed until dissolved. The APTES coated tips were further incubated with the MAL-linker as described for the aldehydelinker. Next $12 \mu \mathrm{g} / \mathrm{ml}(\approx 2 \mu \mathrm{M})$ cysteine mutated EGF was reacted with the slides in degassed PBS buffer ( $\mathrm{pH}$ 7.4) with $1 \mathrm{mM}$ EDTA for 3 hours, and afterwards washed 3 times in PBS. The tips were stored in PBS buffer at $4{ }^{\circ} \mathrm{C}$ for up to 2 days. 


\subsubsection{Immunofluorescence assay}

First, microscopy cover slip \#1.5 glass slides were cleaned by ozone treatment for 30 minutes. Next the glass slides were amino-functionalised and incubated with EGF in a similar way as the AFM tips.

Immunostaining to detect EGF was performed as follows. The slides were first incubated with 5\% BSA for 30 minutes to prevent nonspecific adhesion since not all the slides were passivated by PEG linkers. After washing the slides with PBS, a primary goat antibody specific for the Cterminus of EGF (sc-1341, Santa Cruz, USA) was diluted 1:50 in 0.1\% BSA containing PBS, and the slides were allowed to incubate with this solution for 60 minutes at room temperature. Next the slides were washed 3 times with PBS, and incubated with 1:500 diluted secondary rabbit antigoat antibody labelled with CF-633 (SAB4600148, Sigma-Aldrich) for 60 minutes. After washing with PBS, the slides were mounted on coverslips, and fluorescence microscopy was performed immediately such that the aqueous environment was conserved.

\subsubsection{AFM force spectroscopy}

Force spectroscopy measurements were performed on a Bioscope II AFM (Veeco Instruments Inc.). Force distances curves (typically 1,000) were acquired with a vertical velocity of $200 \mathrm{~nm} / \mathrm{s}$, the z-range was $100 \mathrm{~nm}$, and the maximum deflection force was set at $300 \mathrm{pN}$. MSCT silicon nitride tips B and C from Veeco were used. The nominal spring constant of these tips is $0.01-0.03$ $\mathrm{N} / \mathrm{m}$; effective spring constants of the cantilevers were determined by the thermal noise method in the Veeco software.

\subsubsection{Cell experiments}

Cell culture. All cell culture materials were obtained from PAA Laboratories (Pasching, Austria) unless stated otherwise. The H441 epithelial human lung adenocarcinoma cancer cell line were a gift from Anton Terwisscha van Scheltinga (Department of Medical Oncology, University of Groningen, The Netherlands). H441 cells were cultured in Roswell Park Memorial Institute (RPMI) 1640 medium supplemented with $10 \%$ FBS and Pen Strep at $37^{\circ} \mathrm{C}$ with $5 \% \mathrm{CO}_{2} . \mathrm{H} 441$ cells stably expressing SNAP-ErbB2 at the single molecule level were made by transfecting $\mathrm{H} 441$ cells in a $60 \mathrm{~cm}^{2}$ well of $40 \%$-confluent cells using $6 \mu \mathrm{g}$ of SNAP-ErbB2 plasmid DNA and $12 \mu \mathrm{L}$ Lipofectamine LTX and $6 \mu \mathrm{L}$ Plus reagent (Invitrogen) in $15 \mathrm{~mL}$ Pen Strep free cell medium, as described in the suppliers' protocol. Selection $(500-1,000 \mu \mathrm{g} / \mathrm{mL}$ of G418) was applied after 24 hours and maintained for 12 days. Thereafter the cells were split over 10 wells, and further maintained under selection. After 5 days, one well contained positive cells with an expression level at the single molecule level; these cells were further cultured. For culturing, the concentration of active $\mathrm{G} 418$ was $350 \mu \mathrm{g} / \mathrm{mL}$.

Samples. Before measurements, SNAP-ErbB2 expressing H441 cells were transferred to $35 \mathrm{~mm}$ CellView dishes (product \#627860, Greiner Bio-one, Alphen aan den Rijn, The Netherlands), grown overnight, and then starved overnight the day after transfection in medium without FBS. Labelling of the SNAP-ErbB2 proteins was done by incubating the cells for 2 minutes with $300 \mathrm{nM}$ of BG-Dy549 (SNAP-Surface 549, New England BioLabs, Ipswich, MA, USA) and 500nM of BGCF633 (New England BioLabs, Ipswich, MA, USA) in starvation medium containing 0.5\% BSA. Samples were washed after 2 minutes by replacing the labelling solution with PBS buffer supplemented with magnesium and calcium (PAA Laboratories, product H15-001). This washing step was repeated at least three times, and measurements were performed in the same buffer.

Microscopy. Single-molecule tracking experiments were performed on a single-molecule microscope as described before (Chapter 2-5). The microscope stage was heated to $33-37^{\circ} \mathrm{C}$ with a 
constant current (without feed-back to avoid thermal induced mechanical oscillations) using a sample heating plate and objective ring heater.

\subsubsection{Custom AFM}

A custom AFM was built because the commercially available AFM machines in our lab (Veeco BioScope I and II) were not optimized for force-guided and low-drift indentation experiments. The requirement we had were that the microscope could be equipped with a heating stage to perform experiments at physiological temperatures, and that the AFM tip could be immersed in an aqueous solution. Additionally, the AFM laser should not interfere with the wavelengths used for the fluorescence microscope $(400-720 \mathrm{~nm})$. Furthermore, a slow approach (z-axis) should be possible such that the molecules on the AFM tip are not scratched away upon contact with the cell. This requires that the force feedback should already run whilst using the linear motors during the approach. Upon contact, the force must be kept constant at a preset force. In this phase, the stage (especially in the z-axis) should not have much drift, since the reference force can only be measured before the tip has come into contact with the cell. The realized custom AFM (Fig. 6.3) fulfilled all these requirements, and the z-axis drift was approximately $600 \mathrm{~nm}$ peak-peak per 4 minutes (projected stimulation time), which could be compensated for by the force feedback loop of the AFM since the $\mathrm{Z}$ range of the AFM stage is $20 \mu \mathrm{m}$.

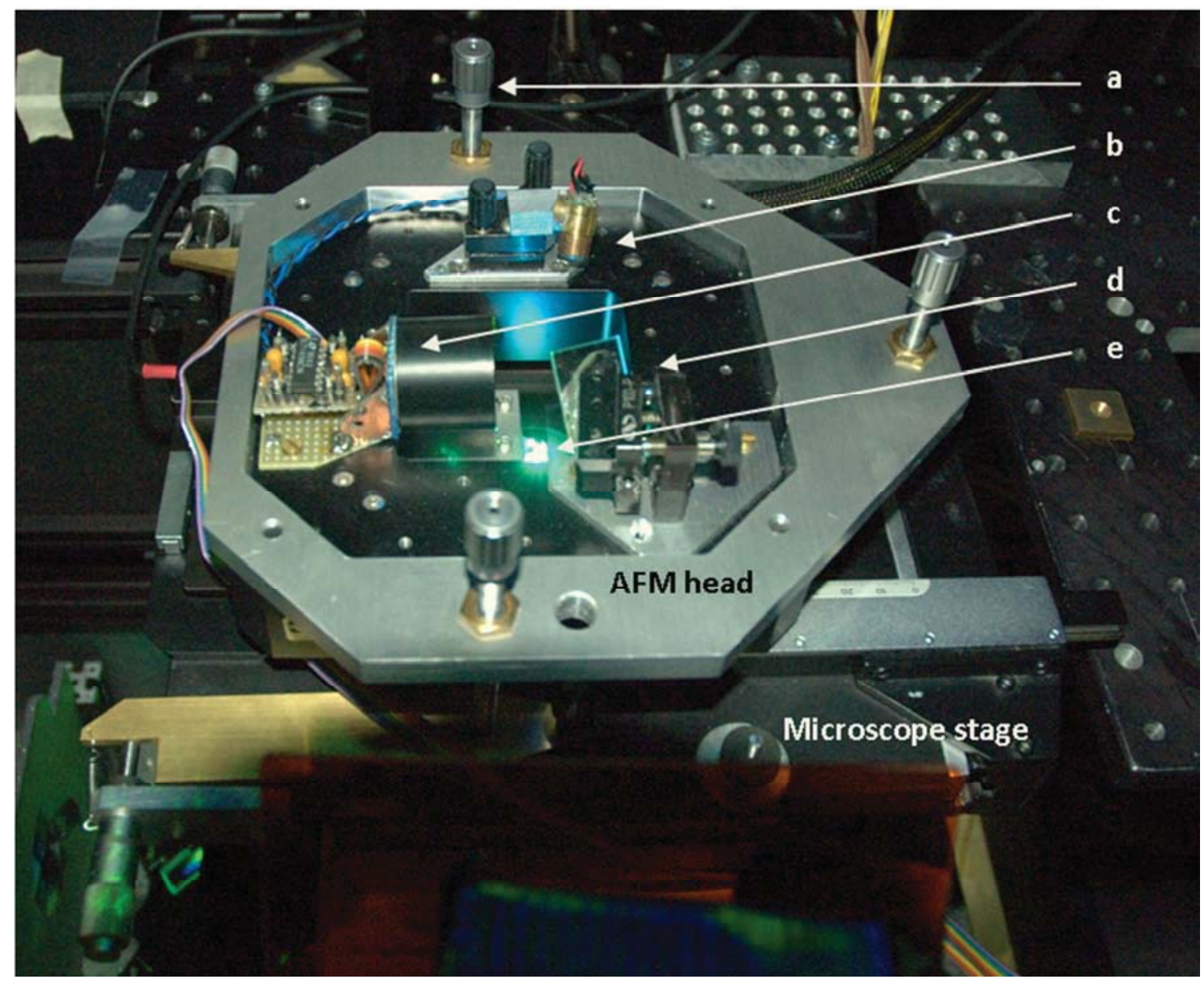

FIGURE 6.3 Photograph of the custom built AFM used in the live cell experiments for local receptor actuation. The AFM head rests on a microscope stage, which can move laterally by the micro positioners on the stage. The micro positioners on the AFM head (a) are to manually adjust the height of the AFM tip above the sample. The AFM laser $(b)$ is focussed on the AFM tip in the tip holder $(e)$, and the reflected light is redirected by an adjustable mirror $(d)$ to the quadrant photodiode $(c)$. 
Further specifications of the custom AFM are now described in more detail (contributed by $R$. Molenaar). The AFM was built on a large aperture piezo nanopositioning stage (Physik Instrumente 517.3CD with E710 controller). The controllable range of this stage is $200 \mu \mathrm{m}$ in lateral direction, and $20 \mu \mathrm{m}$ in axial ( $\mathrm{Z}$ axis) direction. All AFM peripheries were mounted on the scanning body of the stage, i.e. a laser, a cantilever holder, an adjustable mirror and a quadrant photodiode with current-voltage converters. On the fixed body of the stage an aluminium frame (AFM-head) was mounted that holds a tripod configuration of three micro positioners to allow lateral positioning of the AFM tip towards the sample. One tripod micro positioner is motorized to be able to slowly lower the AFM head to the sample (Newport, TRA12CC with SMC100CC controller). The AFM uses a 780nm laser diode (Roithner, LDM780/3LJ), to prevent spectral overlap with the fluorescent channels. A 780nm band-pass filter (Chroma, Z780/30) was placed after the laser diode, to block emission light that would pass into the red channel of the microscope. Another identical bandpass filter was placed in front of the quadrant photodiode to block the light from the excitation lasers used in the setup. The deflection voltage and actuator $\mathrm{Z}$ control voltage are read and controlled by an analog input and output Data Acquisition Card (National Instruments, NI 6353). To have real-time control of the force on the cell membrane, a PI (proportional integral) feedback control loop was implemented in Labview to maintain a preset force.

\subsection{Testing the functionalization of AFM tips with force spectroscopy}

To test the aldehyde-based functionalization chemistry to attach EGF to AFM tips with a heterobifunctional PEG linker, we performed force spectroscopy measurements by recording a series of 1,000 force distance curves. The EGF on the functionalized AFM tip was recognized by antibodies (specific for the C-terminus of EGF) which were bound to a mica surface. The mica surface was functionalized by APTES coating and subsequent antibody binding via the same aldehyde-linker as described for AFM tips. Recorded force distance curves (Fig. 6.4) show zero force when still above the surface, and as the cantilever (the tip) deflects when getting into contact with the surface, this deflection is translated into a deflection force using the cantilever spring constant. The tip is moved towards the surface until the pre-set force limit is reached (trace curve). When the cantilever is subsequently retracted, and no protein binding has occurred or when the surface had been blocked with soluble EGF, the retrace curve will not show a (negative) force while moving the tip upwards away from the surface. When the EGF on the tip has made contact with an antibody on the surface, the retrace curve shows an exponentially increasing negative force upon moving the tip away from the surface. The slope of the deflection force has a typical exponential shape caused by stretching of the PEG linker and the antibody until the binding between EGF and the antibody rapidly ruptures, and the deflection force returns to zero ${ }^{31}$. Consequently, the maximum negative force directly before the rupture event indicates the rupture (unbinding) force of the two proteins. The increase of negative deflection force is expected to start well above the surface, since there is a $10 \mathrm{~nm}$ long PEG linker between the surface and the antibody, then a $10 \mathrm{~nm}$ sized antibody, followed by the EGF, which is attached using the same 10 $\mathrm{nm}$ long PEG linker. Therefore, these specific unbinding events can be distinguished from nonspecific adhesion of the AFM tip itself to the surface.

We recorded several force distance series for slightly differently prepared functionalized AFM tips, and analysed the curves for the typical unbinding characteristics in specific unbinding events (as in Fig. 6.4). We used a typical loading rate of $2,000 \mathrm{pN} / \mathrm{s}$, which is relatively fast to increase the rupture force ${ }^{21}$. As a negative control we performed measurements on a surface that was blocked with soluble EGF. We developed custom software to automatically find the location (z-value) of the surface within the force-distance curve, and predict whether there was a specific unbinding 


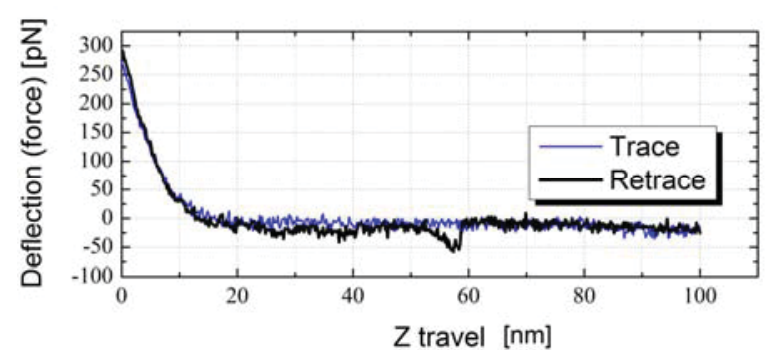

FIGURE 6.4 Example of an force-distance curve, showing the rupture of EGF from an antibody. The PEG linkers cause the typical exponential forcedistance shape and shifts the unbinding event to higher distances compared to nonspecific adhesion. The amino-functionalized tip was functionalized using an aldehyde-PEG-NHS linker and EGF, and an EGF specific antibody was similarly attached to an amino-functionalized mica surface, as shown in Fig 6.2.

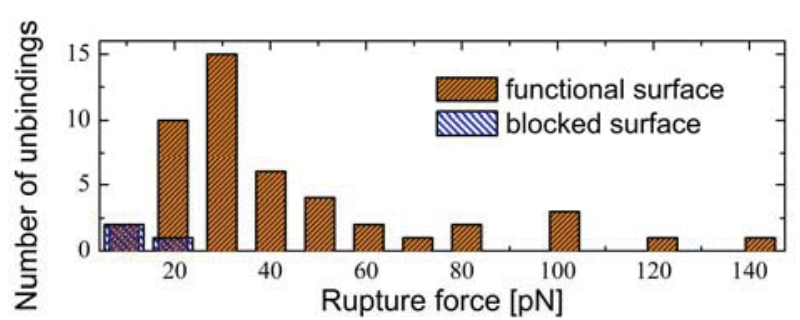

FIGURE 6.5 Probability density function (histogram) of the rupture force of a single EGF from an antibody observed in 47 curves in a series of 750 . The aminofunctionalized tip was functionalized using an aldehyde-PEG-NHS linker and EGF, and an EGF specific antibody was similarly attached to an aminofunctionalized mica surface, as shown in Fig 6.2. With a blocked surface with soluble EGF only 6 out of 750 curves were falsely perceived as specific unbinding events.

event, in which case the exponential force-distance curve and the baseline (zero force) were fitted to obtain the rupture force The fitting was done to an exponential function with variable offset. The user can verify and correct these findings in the software. From these data the unbinding force distributions (a probability density function) were composed. An example of such a distribution (Fig. 6.5), with the lowest recognition percentage for the blocked surface, shows that the unbinding forces are rather low (on the order of the thermal noise levels of the cantilever), and that the specific recognition percentage is rather low (6.3\%).

All fairly reliable measurement series $(\mathrm{N}=10)$ indicated a mean specific unbinding force of 56 $\mathrm{pN}$ with a standard deviation of $42 \mathrm{pN}$. This force compares to individual ligand-receptor recognition forces, which are found to be around 50-250 piconewton ${ }^{15,31-33}$. We obtained a recognition chance of $1-6 \%$ for active surfaces $(\mathrm{N}=10$ series of $500-1,000$ curves), and a chance of $2-5 \%$ for blocked surface. Other antibody-ligand systems prepared using the same protocols showed $25-70 \%$ specific recognition ${ }^{20,27,34}$. In conclusion, our results often show a low recognition chance, which suggests that either there are few tips with a functional EGF molecule present at the tip, or there is not enough antibody present at the recognizing surface, or that either of the molecules fails to make a binding to the other protein.

Since the low percentage of recognition events in numerous series of force spectroscopy experiments did not convince us that we indeed had functionalized our tips with active EGF, we decided to wait until a cysteine mutant of EGF became available. In the mean time we also discovered that the stock antibody solution contained $0.1 \%$ gelatin for stabilization. Gelatin is essentially a large peptide bearing a lot of amino groups. Therefore it is very likely that the recognizing surface did not contain a lot of antibodies against EGF but mostly gelatin molecules. We also decided that although force spectroscopy is a useful technique for single-molecule research on protein forces, it is very time-consuming to just verify the incubation of protein to an APTES layer. Such verification is more easily performed by an immunofluorescence assay with a single-molecule sensitive microscope.

\subsection{Testing the functionalization of AFM tips by an immunofluorescence assay}

An immunofluorescence assay using glass slides instead of AFM tips can serve as a test to confirm binding of EGF to an AFM tip, since the chemistry involved is similar. APTES reacts with 
silicon(di)oxide which is present at both the surface of silicon or silicon nitride AFM tips and glass slides; the first atomic layers of silicon materials are oxidized within days in ambient $\operatorname{air}^{35}$. The main component of both glass slides and AFM tips is therefore silicon(di)oxide. After incubation of cys-EGF on glass slides, using the functionalization chemistry of an AFM tip with a maleimidelinker, and subsequent immunostaining, we recorded fluorescent images on a single-molecule sensitive microscope of both glass slides with cys-EGF and glass slide with APTES only (Fig. 6.6A,E). We also tested for the specificity of EGF binding by excluding the steps with the linker and/or cys-EGF (Fig. 6.6B-D). APTES

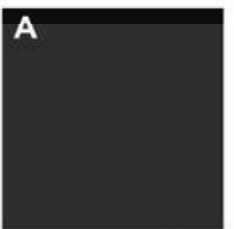

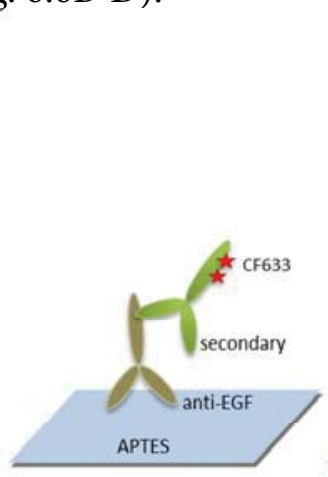

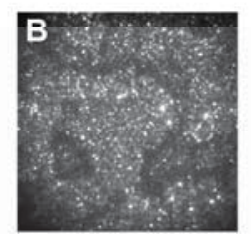

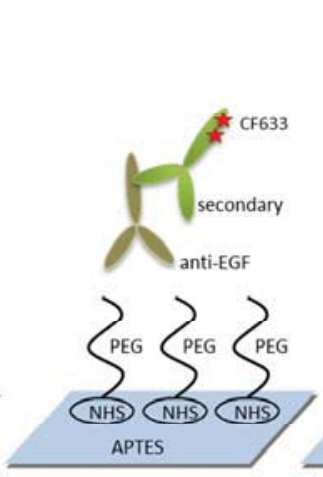

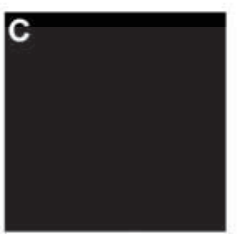

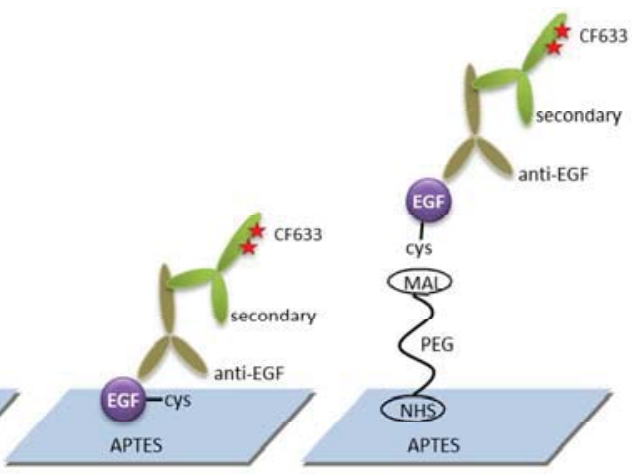
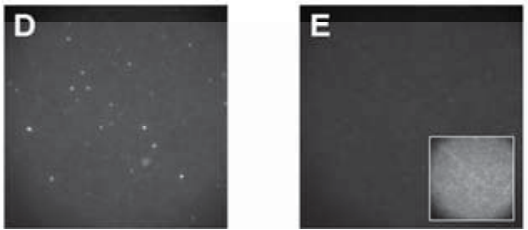

FIGURE 6.6 Incubations performed to show that cys-EGF can be specifically attached via a heterobifunctional PEG-linker onto an AFM tip. In the full procedure, glass slide were coated with an APTES monolayer, incubated with the heterobifunctional NHS-PEG-MAL linker, which can then covalently bind the Nterminal cysteine mutant of EGF. The presence of EGF was confirmed by immunostaining using a polyclonal antibody purified against the C-terminus of EGF. This primary antibody was detected with a CF633 labelled secondary antibody. In $A-D$ some incubations were skipped to confirm that only the full procedure leads to specific attachment without the possibility for nonspecific adhesion of other molecules. The counts indicated are normalised fluorescence intensities of the homogenous background; the spots are neglected. $(A)$ Glass with APTES layer only. Counts: 0. (B) An APTES layer with immunostaining is highly susceptible for nonspecific adhesion of other biomolecules. Counts: 12,000-26,000. (C) Hydrolysing the maleimide (MAL) groups on the PEG linker (by overnight incubation in water) makes the groups unreactive, thereby creating an PEGylated surface on the APTES layer which acts as a passivation layer. Counts: 0, same as APTES only.

(D) The direct non-specific adhesion of cys-EGF and/or antibodies onto the APTES layer shows that to obtain specific labelling a PEG linker is necessary. Counts: 7,000. $(E)$ The full functionalization procedure leads to a homogeneous incubation of EGF on an APTES monolayer. The inset has different intensity scaling and shows that the layer is homogeneously covered. Counts: 5,000 .

The results show that a glass slide with APTES only is not fluorescent. When a glass slide with APTES is immunostained, it shows a high fluorescence signal which is due to nonspecific attachment of the labelled antibody. To avoid nonspecific attachment of biomolecules, surfaces are often passivated with PEG. To replicate the full functionalization procedure, we used the same MAL-PEG-NHS linker but made the maleimide unreactive by subsequent hydrolysis with water. In this case we obtained non-fluorescent slides (Fig. 6.6C). When cys-EGF was nonspecifically attached to the APTES coating without passivation layer (Fig. 6.6D), the slide was fluorescent although not to the same extent as in the case with direct antibody adhesion, possibly due to a complete filling of the nonspecific adhesion sites of the APTES coating by cys-EGF, which are not all oriented such that they are accessible for the antibodies. When the full functionalization 
procedure is followed (Fig. 6.6E), fluorescent images show that the slide is homogenously covered with antibody. Because we can exclude nonspecific binding of antibody due to the PEG linkers, we can conclude that that cys-EGF must be specifically bound through the MAL-PEG-NHS linker to the surface and is homogenously attached to the slide.

\subsection{Selection of an AFM tip for use in single-molecule optical microscopy}

The combination of functionalized AFM tips with optical microscopy requires AFM tips that exhibit low autofluorescence. For this we measured the autofluorescence of three different tips. The tips that were selected have a low spring constant. Because cells are relatively soft materials, the spring constant of the cantilever has to be low $(\approx 0.1 \mathrm{nN} / \mathrm{nm})$ to be able to measure the reacting force of the cellular membrane. The MSCT cantilevers (Veeco at the time of ordering, now Bruker) made of silicon nitride showed very high autofluorescence, although we noticed that the tip itself was not autofluorescent (Fig 6.7A). This tip is undoubtedly made of a different material than the silicon nitride of the cantilever. Nevertheless, the large autofluorescent background observed makes this cantilever unusable for our application. Next we evaluated two tips made of silicon, and brought the tip into contact with a surface incubated with single fluorescent molecules. The CSG01 tips (NT-MDT) and ARROW CONTR tips (NanoWorld) showed virtually no fluorescence (both in the green and red channel, see also chapter 2) compared to the fluorescence of single Dy-549 and CF-633 molecules (Fig 6.7B,C). Therefore these tips were further tested for their suitability to combine with single-molecule fluorescence microscopy. Vibrations due to high-intense illumination power

When a cantilever is positioned in the field of view of the microscope, it absorbs a large amount of the total excitation light ( $\pm 150 \mathrm{~mW}$ combined green and red laser power), which is then converted to heat. Cantilevers are often coated with a reflective layer to increase the reflected light in the photodiode of the AFM. Such layers however make the bending of the AFM cantilever temperature dependent due to bi-metal effects (stress between the two materials). This results in instant deflection responses upon the stroboscopic illumination (to prevent photobleaching between camera acquisition periods). In force feedback, the cantilever deflection, as a measure of the force, is held constant over time. Even though the cantilever heating time response falls within the bandwidth of the feedback of our AFM system, the feedback cannot discriminate between deflection caused by thermally induced deflection (stress inside the cantilever) or height displacement (of the surface). For this reason the force accuracy is limited by the thermal response of the AFM tip and the thermal stability of the stage heater. (Section contributed by R. Molenaar) We measured the deflection force induced by the thermal response when both illumination lasers were used to image single molecules. Whereas CONTR cantilever showed a response that was larger than the measurement range of the photodiode $( \pm 40 \mathrm{nN})$, the CSG01 tips showed a much lower peak-peak force response of $2 \mathrm{nN}$ ). Therefore we continued with the CSG01 cantilever, which has a nominal force constant of $30 \mathrm{pN} / \mathrm{nm}$.

\subsection{Preparation of functionalised AFM tips}

Since the single-molecule recognition events in the force spectroscopy experiments were very sparse or even non-existent, we decided to increase the contact area of the tip with the cells to increase the number of EGF molecules that are exposed to the cell membrane at the same functionalization concentration. This was accomplished by milling the end of the pyramid shaped tip by focussed ion beam (FIB) (Fig. 6.8). The FIB milling did not affect the autofluorescence of the tips (Fig 6.7D). 

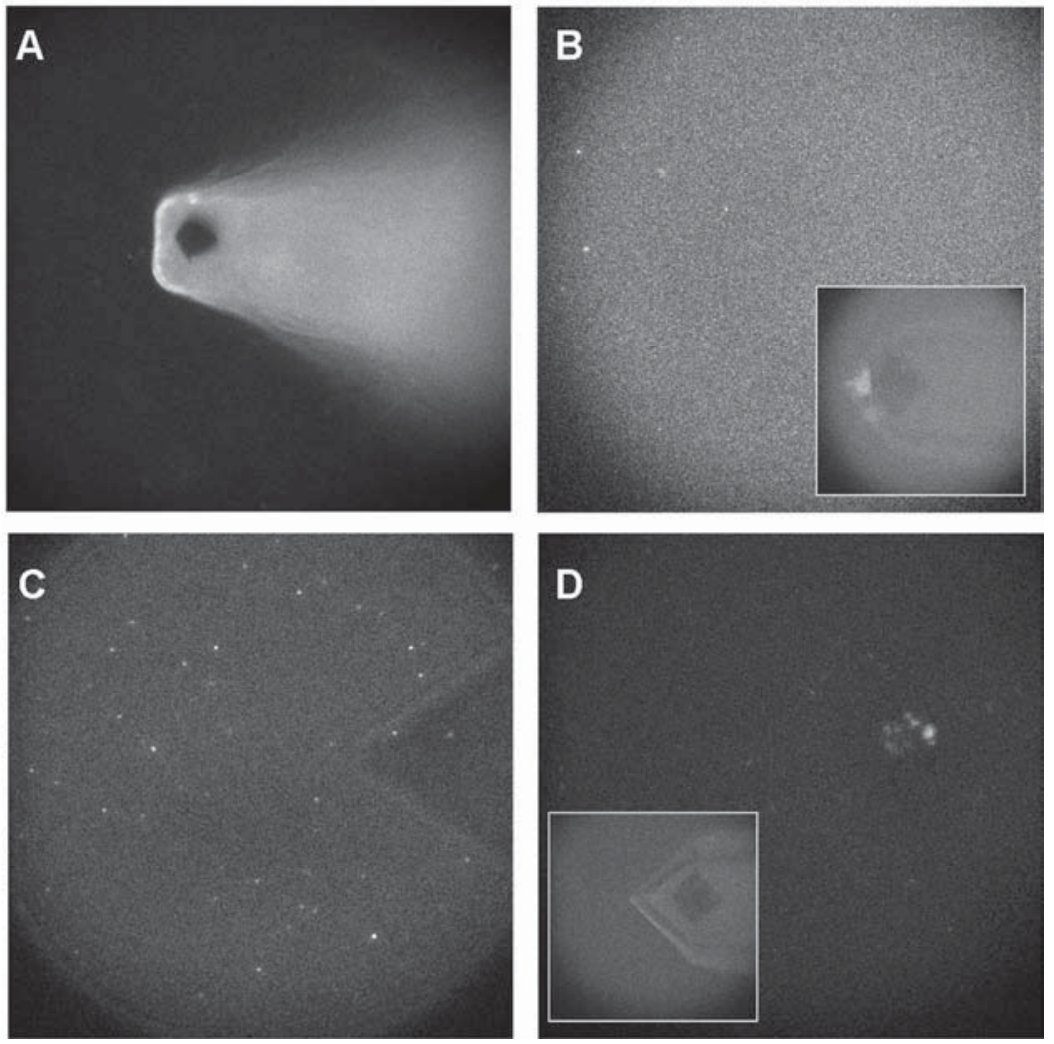

FIGURE 6.7 Fluorescent images of different AFM tips. Tips made of silicon show very little autofluorescence and are suitable for use in simultaneous single-molecule imaging, but tips made of silicon nitride are highly autofluorescent. (A) Tip D of the commonly used MSCT series from Veeco is made of silicon nitride. $(B)$ The NT-MDT CSG01 tip is made of silicon, and the bottom end of this tip is virtually invisible compared to the single molecules on the left of the image. The inset shows that the top of the AFM tip, where it attaches to the cantilever, is vaguely visible. (C) NanoWorld ARROW CONT2 tip made of silicon are also invisible compared to the single molecules. In the combined (weak) white light with fluorescence image shown we find that the single molecules under the tip are easily detectable. (D) NT-MDT CSG01 tip milled by FIB. The milling does not seem to have a large effect on the autofluorescence of the bottom of the tip. The inset shows the top of the tip, which is also fluorescent but very dim.
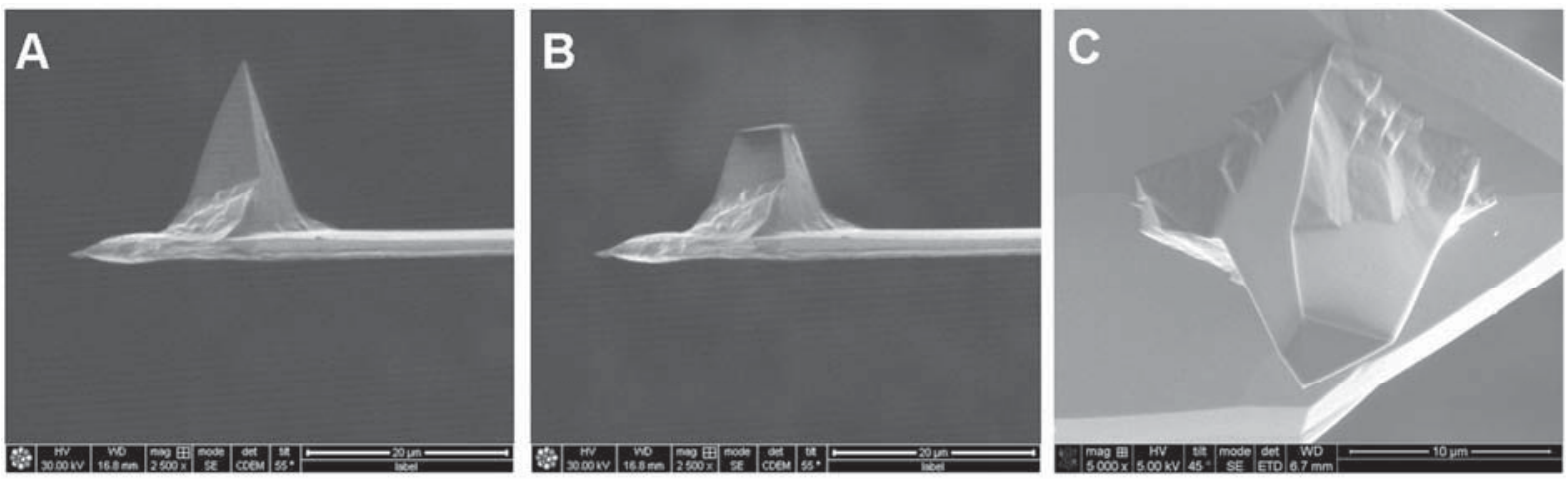

FIGURE 6.8 Electron micrographs of the milling operation by focussed ion beam to obtain a flat surface of approximately $2 \times 2 \mu \mathrm{m}$. (A) The CSG01 tip before milling. $(B)$ The CSG01 tip after milling $(C)$ A closer look at the area created by milling. 
After exposure to ambient air for a few days (to oxidize the silicon surface), milled tips were functionalized with cys-EGF following the procedure with the MAL-linker. For one tip functionalization with EGF was confirmed by immunostaining (as in Fig 6.6E). This tip was mounted in the AFM and brought into contact with the cover glass of a dish containing a PBS solution. Single-molecule fluorescence images of the tip were recorded in both channels (defined in chapter 2). The images (Fig. 6.9) show that EGF was indeed attached to the AFM tip, although we cannot be sure that there are many EGF molecules at the bottom of the pyramid, which is the main contact area with the cell. Because the tip will also bend around the plasma membrane of the cell, we reckoned that the edges of the pyramid of the tip would also get into contact with the cell. Therefore a high local concentration of EGF molecules would be able to access the EGF receptors on the cell membrane, and this batch of functionalised tips was used to perform the live cell experiments. We are not aware of any effect that could have caused this inhomogeneous staining.
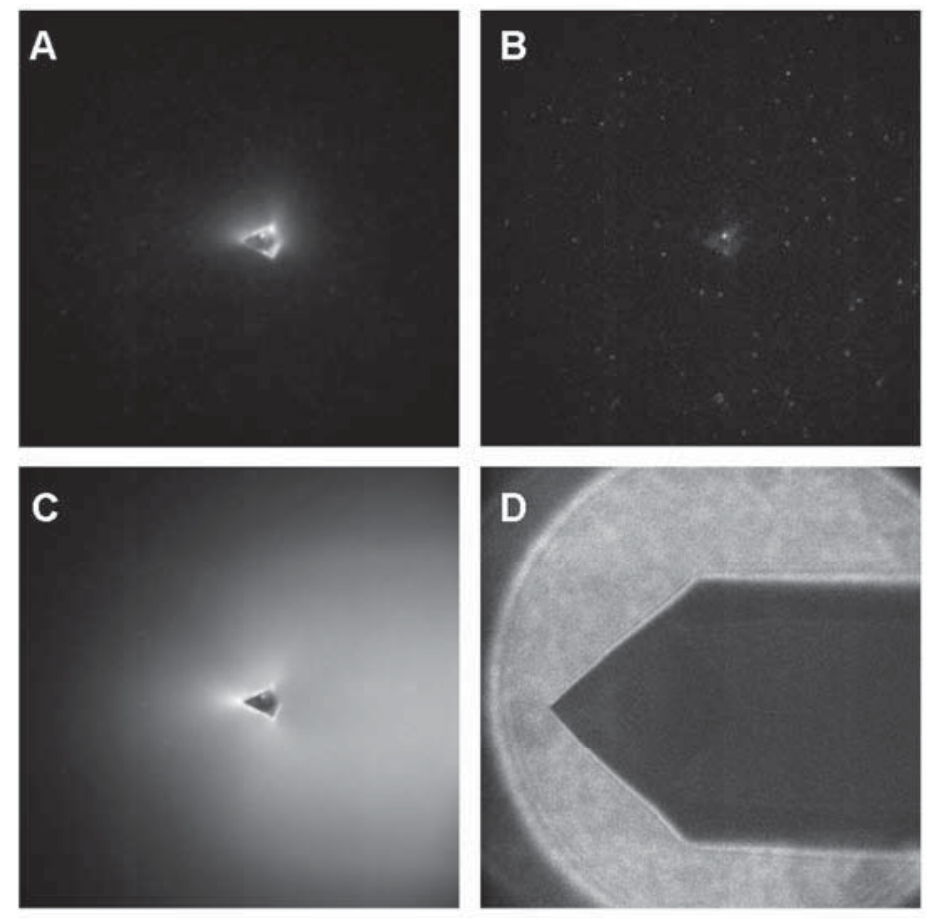

FIGURE 6.9 Fluorescence images of an EGF functionalized and immunostained milled AFM tip. (A) The tip illuminated using TIRF shows that the bottom of the tip is only partly functionalized with EGF (red channel fluorescence image). (B) Fluorescence mage in the green colour channel of the image in panel $A$. The fluorescence observed in A was not autofluorescence of the tip or one of the chemical used as imaging in another colour channel only showed single molecules at the surface (as expected), and no fluorescence from the tip. (C) Fluorescence image of the tip using widefield illumination shows that the edges and sides of the tip pyramid are functionalized. $(D)$ While light image shows the relative position of the functionalised and immunostained AFM tip for panel $A-C$. Images are $60 \times 60 \mu \mathrm{m}$.

\subsection{Preliminary results: cell experiments}

An important aspect of AFM based receptor actuation is to have a microscopy based readout for receptor activation that is compatible with live cells. The early events of EGF receptors activation are kinase phosphorylation of the tyrosine residues. Therefore one possibility is to use a FRET probe to monitor the phosphorylation of the residues, since this is the most direct way to assess the effect of the local actuation. Although FRET between a fluorophores on the receptor and labelled antibody specific for the phosphorylated tyrosine residues of EGFR would yield best results, this approach is incompatible with live cells. Other FRET pairs are based on labelled EGFR and labelled downstream proteins, such as Shc protein, which allows real-time measurements in live cells. However, we were not able to detect FRET between EGFR-mTurquoise and Shc-EYFP ${ }^{36}$ upon EGF addition in HeLa cells using a frequency based fluorescence lifetime imaging microscope (FLIM). Also, we were not able to detect increased FRET with a more optimized pair using phosphotyrosine binding domain (PTB) fused to $\mathrm{EYFP}^{37}$ or PTB-mCitrine (point mutated 
from PTB-Citrine by us). We therefore decided to monitor EGF receptor actuation in a different and more indirect way.

Since the EGF on the AFM tip will bind EGFR, the receptors will be immobilized in the area that is in contact with the AFM tip. When EGFR is activated, the dimerization arm in the extracellular part of the receptor becomes available for dimerization (Chapter 2 and 5). Since ErbB2 is the preferred hetero-dimerization partner of $\mathrm{EGFR}^{38}$, this receptor will also be immobilized when it dimerizes with an EGFR that is bound to the AFM tip via EGF. Therefore such dimerization events can be monitored by tracking ErbB2 receptors by single-molecule fluorescence microscopy during local actuation of EGFR with the AFM tip.

The EGF functionalized milled AFM probes were loaded and brought into contact with H441 cells expressing SNAP-ErbB2 at the single molecule level in PBS. These cells were chosen because they tend to spread quite a lot. This is an advantage when imaging the apical membrane of the cell, the membrane that will get into contact with the AFM tip, while the AFM probe pushes the cell membrane down. The AFM probe has to exert a force to the cell membrane to ensure that it stays in contact with the cell. The H441 cells endogenously express EGFR at a level of about 50 receptors per $\mu \mathrm{m}^{2}$, such that the tip with EGF will have enough receptors to target within the contact area. We gently brought the tip into contact with a cell that had a flat apical membrane such that single molecules could be seen within the focal plane. Upon contact, we recorded fluorescence images of the ErbB2 receptors in the apical plasma membrane of the cells using a TIRF objective with widefield illumination at video-rate ( $24 \mathrm{fps})$. Image series were about 100 frames, and were recorded at an interval of a few minutes, after which we retracted the AFM tip whilst taking fluorescent recordings.

As expected we can see in the supplementary videos that ErbB2 receptors around the contact area indeed became immobile while receptors at a different location in the same cell were still mobile (Fig. 6.10A,B). This effect was almost immediate, and lasted at least for 7 minutes. We believe that the immobilization of the receptors was due to the specific EGF - EGFR - ErbB2 interaction, but we cannot exclude a mechanically induced cytoskeletal effect. Upon retraction of the AFM tip, we noticed membrane bending until $20 \mu \mathrm{m}$ away from the contact area. Furthermore a striking loss of labelled molecules was observed around the contact area (Fig. 6.10C,D). This loss may be due to removal of the molecules that are firmly bound to the AFM tip and pulled out of the membrane upon retraction of the AFM tip. This is supported by the observation that the tip had become fluorescent after being in contact with the cell (supplementary video); the fluorescence spectrum was similar to the spectrum of the dye used to label the ErbB2 receptors. Alternatively the plasma membrane was so much deformed that it was no longer in the same focal plane.

A negative control should confirm whether the SNAP-ErbB2 receptors can still be mobile whilst an passivated AFM tip is touching the cell, such that the receptors are indeed immobilized due to specific stimulation of EGFR by EGF molecules on the AFM tip. The passivated AFM tip may be an APTES coated tip which is passivated with an unreactive PEG linker. The passivation layer is important as an APTES layer or bare silicon suffers from nonspecific interactions with biomolecules (like in Fig. 6.6A). Another study already showed that PEG linkers on the AFM tip do not interact with or penetrate into the lipid membrane of living cells ${ }^{25}$. 

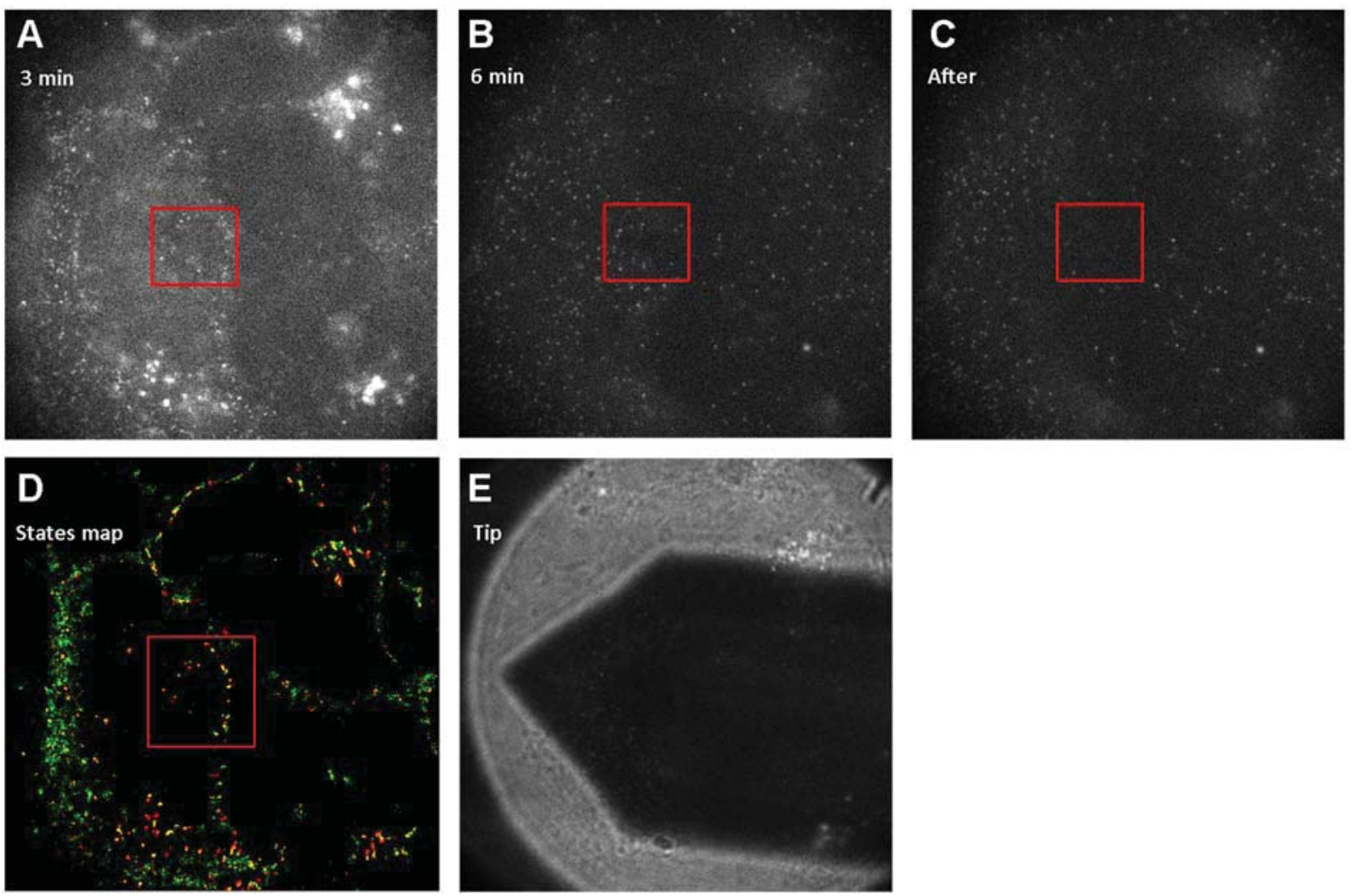

FIGURE 6.10 Nano-actuation of EGFR in H441 cells with an EGF-functionalised AFM tip. The fluorescent images show individual labelled SNAP-ErbB2 receptors, which are hetero-dimerization partner for EGFR. $(A)$ After 3 minutes of tip-cell contact (red squared box), the labelled molecules at the contact area have all become immobile, whereas the labelled molecules away from the contact area, e.g. at the left side of the cell, are still mobile like before the tip came into contact. (B) After 6 minutes of tip-cell contact (red squared box), the AFM tip has pushed itself through the cell and has reached the basal membrane. The molecules at the contact area are still immobile unlike the molecules further away. $(C)$ When the tip was retracted (gone out of contact), the cell was deformed over a large area, and the labelled molecules around the contact area disappeared. $(D)$ A diffusion state map composed from the trajectories of the video after 3 minutes of tip-cell contact. This state map was composed as detailed in Chapter 3 using the gyration measure. At the tip-contact area there are almost only molecules residing in a slow or immobile state, whereas at the left hand side of the cell there are a lot of molecules still mostly residing in a mobile state. Although there are more regions of strongly reduced diffusion in the same cell, these areas might have contained more vesicles (panel A), which could be a reason for the mostly reduced diffusion there. $(E)$ The position of the functionalised AFM tip is at the right side of the cell on the left of the field of view (white light recording). The tip is still above the cells. All images are $60 \times 60 \mu \mathrm{m}$.

\subsection{Conclusions}

The interactions of ligands with plasma membrane proteins is widely studied, but almost never in a way where there is exact control over the duration of application and precise location of the stimulus. We have demonstrated that ligand functionalized AFM tips can be used to realize such experiments. To ensure that ligands induce receptor actuation, we enlarged the tip-cell contact area by milling the bottom part of the AFM tip by focussed ion beam. We have seen that ErbB2 receptors, which are the preferred dimerization partner of EGFR, became immobile around the tip-cell contact area whereas ErbB2 receptors at a different location in the same cell were still mobile. A negative control is still required to confirm the specificity of the interaction.

To obtain EGF functionalized AFM tips proved to be a difficult task with wild type human EGF, which does not have free accessible thiol groups and whose lysines are partly located in the 
To obtain EGF functionalized AFM tips proved to be a difficult task with wild type human EGF, which does not have free accessible thiol groups and whose lysines are partly located in the binding pocket. This might be due to the slow or low binding chance of the $\mathrm{N}$-terminal amino group to the aldehyde group of the aldehyde-linker at $\mathrm{pH}$ 5.5, or due to the gelatin in the solution of the recognizing antibody incubated on the recognizing surface in the force spectroscopy experiments. However, we did not have access to a cysteine mutant of EGF at that time. For testing the binding chemistry of the mutant, we devised an immunofluorescence assay that mimics the functionalization chemistry to the silicon oxide in the AFM tips by using silicon (di)oxide glass slides instead. This assay was not prone to amino groups contaminations in the antibody solution, since these antibodies were not bound via an aldehyde group as necessary in the force spectroscopy measurements. The assay showed that we can achieve a successful attachment of cysteine mutated EGF to an AFM tip.

Ultimately, the goal of single-molecule manipulation is to be in command of the signalling machinery of a single receptor in a living cell. By studying the behaviour of molecular machines one by one, we might infer the design principles for cellular decision making and outcome. Combining advanced optical techniques with chemical force probe microscopy has only recently come into focus of the scientific community as a new toolbox to unravel single molecule interactions. Combining single-molecule tracking fluorescence microscopy with functionalized AFM for locally actuating plasma membrane receptors will permit a combined visualization and manipulation of these receptor down to nanoscopic scales. The AFM probes may present different chemical stimuli to the receptors, such as ligands but also inhibitory antibodies, or yet another class of molecules. The further development of such biophysical tools will help us to cross borders currently limited by conventional microscopy techniques, and to elucidate the sophisticated functions of biological matter.

\section{Acknowledgment}

We thank Robert Molenaar for the implementation, characterization and documentation of the custom built AFM and controlling software. We thank Kees van der Werf for the initial design of the custom built AFM. We thank Jenny Ibach for providing SNAP-ErbB2 plasmid DNA, Donna Arndt-Jovin for providing Shc-EYFP, and Philippe Bastiaens for providing PTB-Citrine. We thank Andreas Ebner for providing NHS-PEG-aldehyde crosslinkers and discussions on forcespectroscopy measurements. 


\subsection{References}

1. Bustamante, C., Macosko, J. C. \& Wuite, G. J. Grabbing the cat by the tail: manipulating molecules one by one. Nat. Rev. Mol. Cell Biol. 1, 130-6 (2000).

2. Dupres, V., Alsteens, D., Andre, G., Verbelen, C. \& Dufrêne, Y. F. Fishing single molecules on live cells. Nano Today 4, 262-268 (2009).

3. Kapanidis, A. N. \& Strick, T. Biology, one molecule at a time. Trends Biochem. Sci. 34, 234-43 (2009).

4. Kufer, S. K., Puchner, E. M., Gumpp, H., Gaub, H. E. \& Liedl, T. Single-molecule cut-and-paste surface assembly. Science 319, 594-6 (2008).

5. Verveer, P. J. Quantitative Imaging of Lateral ErbB1 Receptor Signal Propagation in the Plasma Membrane. Science 290, 1567-1570 (2000).

6. Schlessinger, J. All Signaling Is Local? Mol. Cell 10, 218-219 (2002).

7. Reynolds, A. R., Tischer, C., Verveer, P. J., Rocks, O. \& Bastiaens, P. I. H. EGFR activation coupled to inhibition of tyrosine phosphatases causes lateral signal propagation. Nat. Cell Biol. 5, 447-53 (2003).

8. Lemmon, M. A. \& Schlessinger, J. Cell signaling by receptor tyrosine kinases. Cell 141, 1117-34 (2010).

9. Deniz, A. A., Mukhopadhyay, S. \& Lemke, E. A. Single-molecule biophysics: at the interface of biology, physics and chemistry. J. R. Soc. Interface $\mathbf{5}$ 15-45 (2008)

10. Lamontagne, C.-A., Cuerrier, C. M. \& Grandbois, M. AFM as a tool to probe and manipulate cellular processes. Pflugers Arch. 456, 61-70 (2008).

11. Lee, S., Mandic, J. \& Van Vliet, K. J. Chemomechanical mapping of ligand-receptor binding kinetics on cells. Proc. Natl. Acad. Sci. U. S. A. 104, 9609-14 (2007).

12. $\mathrm{Li}, \mathrm{G} ., \mathrm{Xi}, \mathrm{N}$. \& Wang, D. H. Probing membrane proteins using atomic force microscopy. J. Cell. Biochem. 97, 1191-7 (2006).

13. Müller, D. J., Krieg, M., Alsteens, D. \& Dufrêne, Y. F. New frontiers in atomic force microscopy: analyzing interactions from single-molecules to cells. Curr. Opin. Biotechnol. 20, 4-13 (2009).

14. Müller, D. \& Dufrêne, Y. Atomic force microscopy as a multifunctional molecular toolbox in nanobiotechnology. Nat. Nanotechnol. 3, 261-269 (2008).

15. Chtcheglova, L. A., Waschke, J., Wildling, L., Drenckhahn, D. \& Hinterdorfer, P. Nano-scale dynamic recognition imaging on vascular endothelial cells. Biophys. J. 93, L11-3 (2007).

16. Chtcheglova, L. A., Wildling, L., Waschke, J., Drenckhahn, D. \& Hinterdorfer, P. AFM functional imaging on vascular endothelial cells. J. Mol. Recognit. 23, 589-96 (2010).

17. Madl, J. et al. A combined optical and atomic force microscope for live cell investigations.

Ultramicroscopy 106, 645-51 (2006).

18. Han, S.-W. et al. Successive detection of insulin-like growth factor-II bound to receptors on a living cell surface using an AFM. J. Mol. Recognit. 24, 17-22 (2011).

19. Duman, M. et al. Nanomapping of CD1d-glycolipid complexes on THP1 cells by using simultaneous topography and recognition imaging. J. Mol. Recognit. 26, 408-14 (2013)

20. Ebner, A., Hinterdorfer, P. \& Gruber, H. J. Comparison of different aminofunctionalization strategies for attachment of single antibodies to AFM cantilevers. Ultramicroscopy 107, 922-7 (2007).
21. Ebner, A. et al. in Handbook of Single-Molecule Biophysics 407-447 (Springer, 2009). doi:10.1007/978-0-387-76497-9_15

22. Puntheeranurak, T., Neundlinger, I., Kinne, R. K. H. \& Hinterdorfer, P. Single-molecule recognition force spectroscopy of transmembrane transporters on living cells. Nat. Protoc. 6, 1443-52 (2011).

23. Bippes, C. a \& Muller, D. J. High-resolution atomic force microscopy and spectroscopy of native membrane proteins. Reports Prog. Phys. 74, 086601 (2011).

24. Te Riet, J. et al. Distinct kinetic and mechanical properties govern ALCAM-mediated interactions as shown by single-molecule force spectroscopy. J. Cell Sci. 120, 3965-76 (2007).

25. Wildling, L. et al. Probing binding pocket of serotonin transporter by single molecular force spectroscopy on living cells. J. Biol. Chem. 287, 105-13 (2012).

26. Posch, S. et al. Activation induced morphological changes and integrin $\alpha_{\|}{ }_{1 b} \beta_{3}$ activity of living platelets. Methods 60, 179-85 (2013).

27. Riener, C. K. et al. Simple test system for single molecule recognition force microscopy. Anal. Chim. Acta 479, 59-75 (2003).

28. Crampton, N., Bonass, W. a, Kirkham, J. \& Thomson, N. H. Formation of aminosilanefunctionalized mica for atomic force microscopy imaging of DNA. Langmuir 21, 7884-91 (2005).

29. Ebner, A. et al. A new, simple method for linking of antibodies to atomic force microscopy tips. Bioconjug. Chem. 18, 1176-1184 (2007).

30. Lee, H., Jang, I. H., Ryu, S. H. \& Park, T. G. Nterminal site-specific mono-PEGylation of epidermal growth factor. Pharm. Res. 20, 818-25 (2003).

31. Hinterdorfer, P., Baumgartner, W., Gruber, H. J., Schilcher, K. \& Schindler, H. Detection and localization of individual antibody-antigen recognition events by atomic force microscopy. Proc. Natl. Acad. Sci. U. S. A. 93, 3477-81 (1996).

32. Almqvist, N. et al. Elasticity and adhesion force mapping reveals real-time clustering of growth factor receptors and associated changes in local cellular rheological properties. Biophys. J. 86, 1753-62 (2004).

33. Lee, G. U., Kidwell, D. A. \& Colton, R. J. Sensing Discrete Streptavidin-Biotin Interactions with Atomic Force Microscopy. Langmuir 10, 354-357 (1994).

34. Stroh, C. M. et al. Simultaneous topography and recognition imaging using force microscopy. Biophys. J. 87, 1981-90 (2004).

35. Mende, G. Oxidation of etched silicon in air at room temperature; Measurements with ultrasoft X-ray photoelectron spectroscopy (ESCA) and neutron activation analysis. Surf. Sci. 128, 169-175 (1983).

36. Sorkin, a. Internalization of the epidermal growth factor receptor: role in signalling. Biochem. Soc. Trans. 29, 480-4 (2001).

37. Offterdinger, M., Georget, V., Girod, A. \& Bastiaens, P. I. H. Imaging phosphorylation dynamics of the epidermal growth factor receptor. J. Biol. Chem. 279, 36972-81 (2004)

38. Graus-Porta, D., Beerli, R. R., Daly, J. M. \& Hynes, N. E. ErbB-2, the preferred heterodimerization partner of all ErbB receptors, is a mediator of lateral signaling. EMBO J. 16, 1647-55 (1997). 



\section{Chapter 7}

General discussion 
Single-molecule tracking has not yet been used to its fullest potential to reveal elusive details of the proteins in the plasma membrane. Nevertheless, the potentials of the technique are almost endless, ranging from following the lifespan of a protein, to finding clues of the composition of the plasma membrane ${ }^{1}$. Extending tracking to $3 \mathrm{D}$ using double-helix microscopy also allows to observe the lifespan of cytosolic proteins ${ }^{2-4}$. Especially promising is the use of multiple colours to track multiple proteins, which allows direct observation of homo- and hetero-dimerization of single receptors $^{5,6}$ and interactions of cytosolic proteins when tracking in 3D. Understanding proteinprotein interactions is key to understand the kinetics in signal transduction ${ }^{7}$. In this chapter we provide an overview of the relevant new instrumentation developments and scientific insights gained described in the course of this thesis. Scientific work usually brings more questions than answers. Therefore we also provide a number of future perspectives and discuss some of the hurdles of performing single-molecule tracking experiments.

The single-molecule tracking technique is not yet as much used as other microscopy techniques. However, once a protein can be suitably labelled and advanced analysis toolboxes can be applied on the trajectories obtained, the time required to prepare and perform the experiments and subsequent analysis is about equal. However, single-molecule tracking has more potential and clear advantages over other microscopy techniques, such as FRAP measurements (described in chapter 1). We therefore envision that single-molecule tracking might become more and more a standard microscopy technique in biological and medical research.

The first stage of the research described was to realize a single-molecule sensitive microscope (chapter 2), which can image individual fluorescently labelled proteins. We utilized low-cost highpower $( \pm 0.5 \mathrm{Watt})$ solid-state lasers to obtain relatively high illumination power in single cells resulting in detectable fluorescent signals at acquisition times of around a millisecond for optimized labels. At that time most single-molecule microscope were build using continuouswave (CW) gas lasers where acousto-optical tunable filters (AOTF) can switch the illumination light on and off. We realized that solid-state lasers could deliver much more power relative to their price than gas lasers (per laserline). Although low-cost lasers have rarely been used before for single-molecule imaging, and do not typically have a beam quality and coherence length as good as more expensive gas and solid-state lasers, we showed that these properties are not crucial to realize a highly-efficient microscope yielding good image quality to detect the signals from weakly emitting fluorophores. Much more important is the choice of a good filter set. We envision that future single-molecule microscopes will be realized more using solid-state lasers due to the costeffectiveness of this solution and their ease of use and modularity. Indeed contemporary commercial STORM microscopes already make use of solid-state lasers. Nowadays it is also possible to buy a complete set of high-power RGB solid-state lasers $(635 \mathrm{~nm} / 532 \mathrm{~nm} / 473 \mathrm{~nm})$, such that no additional alignment of the laser beams is required. We have also introduced the use of a rotating diffuser to reduce interference effects in the illumination pattern. A diffuser based method allows working in free-space, i.e. without fibres, which makes optical adjustments easier and in practice results in less loss of optical illumination power.

This thesis has shown that whereas the current instrumentation available to build a singlemolecule tracking setup has almost ideal specifications, mainly methodological hurdles are obstacles in advancement of the technique. Especially limiting to derive convincing conclusions on protein clustering is the lack of an ideal protein labelling system to date to obtain unobscured multiple-colour data. Ideally, an orthogonal labelling system, which allows multiple protein species to be labelled, is used with the best fluorescent labels available. The ideal fluorescent label for single-molecule tracking would be a bright and photostable dye, with an ultimate specificity for the protein of interest, monomeric and non-adhesive such that no artificial clustering can be induced; it should also be of small size to avoid a loss of functionality due to steric hindrance for conformational changes of the protein, or due to an increased drag in a crowded environment. Protein-tag labelling systems, such as SNAP-tag labelled with organic dyes, which can be 
combined with CLIP-tag for orthogonal labelling, are a promising labelling system. Whereas such tags can be labelled with an optimal choice of fluorescent substrates in respect to brightness and specificity, the amount of blinking and relatively rapid photobleaching of single dyes keeps this system far from ideal yet. In Chapter 4 we showed that SNAP-tag fused proteins can be labelled at the single-molecule level in two distinct colours by BG-Dy549 and BG-CF633. Although this is not the first demonstration of using two SNAP substrates to obtain dual-colour labelling of SNAP$\operatorname{tag}^{8}$, our choice of fluorophores allows for a prolonged imaging period without much blinking, unlike earlier choices. In particular, choosing a red-excitable dye that did not suffer from photobleaching or nonspecific attachment required an extensive evaluation of fluorophores beyond the ones used earlier to label SNAP-tag.

Single-molecule tracking requires exceptionally long trajectories to unambiguously observe dimerization in multiple distinct colours of labels. Even though quantum dots are extremely bright and photostable, they are much larger than most proteins themselves which might sterically hinder the movement of the protein'. This reduces the applicability of quantum dots to single molecule tracking microscopy when monitoring the diffusion of proteins. The mobility of proteins is also important for their unperturbed function when monitoring protein interactions. When studying clustering effects of receptors, monovalent quantum dots should be used, which requires custom fabrication and optimization ${ }^{10-12}$. Even when no differences in mobility are observed after labelling a protein with a quantum dot or a smaller label, quantum dots might result in artificial induction or stabilization of dimers due to their tendency for nonspecific labelling and aggregation ${ }^{13,14}$. This tendency certainly enlarges the chance to see a labelled dimer in an environment with numerous unlabelled receptors (forming only transient dimers). In contrast, the SNAP-tag and the fluorescent labels used here do not have a tendency to adhere nonspecifically (chapter 4), and we did not observe correlated motion of labelled receptors in the dual-colour recordings (also hours after labelling) which indicates that there is no tendency for agglomeration either (chapter 5). Dimerization events probably failed to appear due to the high number of non-labelled endogenous ErbB receptors. However, others have seen dimers and even altered dimer lifetimes when utilizing quantum dots in HeLa cells, which endogenously express a large number of ErbB receptors with respect to the labelled number of receptors ${ }^{5}$. This should have made it unlikely to observe dimers by correlated motion in that cell line. Dual-colour correlated motion experiments for EGFR are perhaps better performed in cell lines which contain no endogenous ErbB receptors, such as Chinese hamster ovary (CHO)-K1 cells ${ }^{15}$. However in that case a hamster cell line is used to draw conclusions about human receptors. When correlated motion is indeed observed in such cell lines using SNAP-tag labels, this is an indication that the SNAP labelling system using organic dyes is more suitable to detect dimers. To conclude, it is important to determine the aggregation tendency of a label, as labels might induce or stabilize dimerization of proteins.

It is crucial to find better fluorescent labels that are getting close to the ideal label than currently available labels to study the lifetimes of dimers. Further fluorescent label developments, such as highly stable organic dyes and small nano-diamonds ${ }^{16}$ and discotics ${ }^{17}$, are therefore essential to further advance the capabilities of single-molecule tracking. We emphasize that quantum dots can be effectively used for tracking applications where their aggregation tendency is not a concern, such as looking at altered motion of a protein due to a certain cellular structure.

Another hurdle in the advancement is the difficulty to relate protein motion to biological implications. Whereas multiple colour tracking holds clear promise in unravelling protein-protein interactions, the motion itself also holds a lot of hidden information. One approach to extract the information from protein trajectories is to fit MSD curves of complete trajectories on models as commonly performed. Whereas this is a valid approach when the motion is homogenous, several reports have showed that protein motion is not homogenous but includes transient effects ${ }^{1,18-21}$. We believe that the transient nature of protein motion requires other analysis methods, and that 
the use of full trajectory analyses should be limited when the state lifetimes are much shorter than the trajectory recorded. Although difficult, because diffusion leads to a large spread of displacements, it is not impossible to analyse short trajectory segments which have much less overlap of diffusion states.

Chapter 3 described the framework composed to perform a classification of dynamical diffusion states from protein trajectories. Currently there is no generally accepted method to perform such a task. The novelty in our framework is the notion that there is no need to determine local diffusion values for segments, since the global diffusion values can be very accurately obtained from a fit using all displacements in the trajectories. The approach can therefore use an objective threshold for diffusion classification using any quantification measure of choice. Different quantification measures currently used to characterize the motion of proteins can be used in this framework, but confinement measures had not previously been used to provide a classification of diffusion states. Our framework allows to utilize the robust gyration method to objectively classify segments to a diffusion state. Moreover, the visualization of where proteins move slowly has heretofore not been combined with super-resolution like images. Our framework and other methods recognizing the different transient modes of protein motion are of general use for the analysis of single protein tracking data. Our approach has already showed its potential to reveal protein interactions with cellular components such as microtubules and filopodia.

We showed that the spatial information of protein motion can reveal where proteins interact with cellular structures, since binding of proteins to such structures alters their diffusion speed. Whereas this spatiotemporal information did not show many features at first sight in the case of EGFR, remarkably clear elongated structures were visible in the case of EP4 (manuscript in review), a prostanoid $\mathrm{G}$ protein-coupled receptor. The visualization of the diffusion states at high spatial resolution as developed in chapter 3 was essential to reveal the mechanism regulating the relatively rapid signalling by EP4 upon ligand binding. In contrast, EP2, a related GPCR, shows marginal signalling and does not localize to the elongated areas. The dynamically localization of EP4 in elongated areas of the plasma membrane were found to be structured by the underlying cortical microtubule network. Especially interesting was that EP4 exhibited almost exclusively slow diffusion near microtubules, where these structures are normally associated with active transport. This led to an additional concept of the dynamic organizations of proteins in the plasma membrane, different from actin corrals and lipid rafts, based on cortical microtubules as template hubs for receptor signalling initiation and attenuation. Visualization of the diffusion states of other membrane receptors might reveal more details about the mechanisms orchestrating receptor signalling.

We assumed that the motion of plasma membrane receptors, such as EGFR, is described by a two population diffusion system, which is probably too naive. In fact, we noticed that several molecules showed rather linear movements, probably due to active transport by microtubules or actin orchestrated paths (chapter 3 and 5), and certainly in filopodial structures in the cell periphery. These linear movements are probably even noticeable in the histograms of squared displacements as a small bump of larger displacements. Therefore our framework could be expanded to extract such linear transport first, for example by looking at the difference in the direction between subsequent displacements in a segment, and only classify the remaining displacements by populations of diffusion.

Other future applications for single-molecule tracking and analysis of diffusion and receptor dimerization might lie in the development of anti-cancer drugs. A crucial step in the development of a novel anti-cancer drug is to acquire molecular evidence for drug functionality. A combination therapy, where more than one domain of the protein or even multiple proteins are simultaneously targeted, seems to have most promises for therapeutic success ${ }^{22}$. However, due to the vast number of possible combinations of drug combinations, more ways to acquire insight in the molecular effects of drugs are needed. In chapter 5 we showed that reduced diffusion speeds obtained using 
single-molecule tracking may assist in finding clues about receptor dimerization. Once a protein system can be adequately labelled, experiments to study the reduced diffusion upon addition of many different drugs and combinations of drugs can be performed in a relatively straight-forward manner.

Tracking of EGFR to obtain insights in its motion, its reliance on lipid domains in the plasma membrane, and on dimerization has been the subject of extensive previous study ${ }^{5,23-27}$. In Chapter $\underline{5}$ we described the diffusion of EGFR in terms of a two population diffusion system, which therefore does not give an average description of the motion within a trajectory, but a description of a diffusion system which allows for transitions between states within the trajectories. Such descriptions are rarely given, and only once recently for EGFR ${ }^{5}$. We have, in the context of the two-population diffusion model developed in this work, studied the influence of the receptor antagonists erlotinib, lapatinib and cetuximab on the motion of EGFR and ErbB3. We noticed alterations in the diffusion speed that could be related to dimerization, which may have consequences for the molecular design of drug molecules. Although speculative, our results provide evidence that ErbB3 is related to EGF-induced EGFR signalling by leaving inactive clusters and forming hetero-dimers with EGFR upon conformational changes induced by cetuximab of EGFR.

As a final excursion, we have demonstrated that functionalized AFM tips can be combined with single-molecule fluorescence microscopy, such that single receptors on the plasma membrane can be tracked whilst locally actuating a subpopulation of receptors. With an EGF functionalised tip we showed that receptors (ErbB2) are halting their motion when in low-pressure contact with the tip (contact force around $1.5 \mathrm{nN}$ ). We might have observed specific hetero-dimerization events, which would have resulted in this halt of motion of the dimerization partner of the receptor actuated (chapter 6). However, the availability of a good readout probe for phosphorylation of the particular receptor actuated in live cells remains essential to truly understand the implication of the stimulus in such experiments. Whereas the idea to bring rationally designed actuating probes in a controlled fashion to the cell sounds appealing, its value to answer biological questions has to be carefully considered. A unique aspect of AFM induced receptor actuation is the possibility to retract the AFM tip to obtain a short stimulation impulse, such that the impulse response of receptor signalling can be measured. Such information can provide more details of the highly complex biological feedback loops, but requires readout probes for the processes in the signalling cascade. Furthermore, the possibly attenuated signalling strength of a relatively short lived ligand stimulation can yield interesting results, as cells also have an intrinsic signal attenuation system (ubiquitination and degradation). From a biological and medical perspective, studying and manipulating that attenuation system can be an interesting new route in anticancer research, such as developing a drug molecule that rapidly ubiquitinates (and degrades) a receptor upon ligand binding.

To conclude, there is enough room for improvement in the toolbox for fluorescently labelling proteins at the single-molecule level and to extend the analysis toolbox for protein tracking data to encourage biophysicists to further advance and develop the single-molecule tracking technique to unleash its full potential. 

Summary 
In this thesis we developed tools to utilize single-molecule tracking microscopy to study the signalling mechanism of the ErbB family of receptors, and in particular of the epidermal growth factor receptor (EGFR). This receptor family is prototypical of receptor tyrosine kinases (RTK) and are implicated in the development and progression of various human cancers, and therefore form attractive targets for drug discovery. The organisation of these receptors in the plasma membrane shows dynamic clustering and hindered motion on the nano- and micro-scale levels. This dynamical behaviour of proteins are thought to play a crucial mechanistic role in the amplification and sensitivity of signal transduction inside the cell. We related the diffusion speed of EGFR to its dimerization state, which is an essential step in its central role of transducing extracellular signals into cellular outcomes. Malignancies therein and drug-induced dimerizations are therefore important research topics for therapeutic application.

Our single-molecule tracking data of EGFR and ErbB3 revealed specific molecular interactions in tumour cells, and also altered behaviour when drugs are applied which are especially designed to prevent such molecular interactions. Before we were able to record trajectories of EGFR, and analyse these in term of dimerization and interactions with other cellular structures, we realized a microscope for this purpose, devised a framework to analyse trajectories in term of different diffusion populations, and further advanced an existing protein labelling system - SNAP-tag - to the single molecule level. The research described in this thesis offers advancements to this proteintag labelling system for application in single-molecule imaging and tracking, and to the postmeasurement analysis of protein trajectories recorded by introducing a classification framework for multiple populations of diffusion, to be able to investigate dynamic protein motion and interactions in live cells. We concluded our thesis with a proof of principle that locally induced stimulation of receptor by ligand functionalized AFM tips can provide additional insight in molecular interactions when combined with single-molecule tracking.

Chapter 1 provides an introduction to the ErbB family of receptors and their molecular interactions; the single-molecule tracking technique in general and currently available fluorescent labels; and to controlled receptor actuating tools focussing on functionalization strategies of AFM cantilevers. In chapter 2 , we describe the requirements and implementation for a single molecule sensitive fluorescence microscope to be able to track single fluorescently labelled proteins in live cells. We demonstrated that solid-state lasers are a cost-effective alternative to widely used gas lasers for this application, without compromising image quality. A rotating diffuser and Köhler illumination scheme were used to provide high-intensity incoherent excitation light without the need for a more common fibre coupling. The realized setup can detect proteins labelled with single fluorescent organic dyes with a localization accuracy typically down to $20 \mathrm{~nm}$ within $10 \mathrm{~ms}$, which satisfied the requirements to track EGFR. To construct protein trajectories from the recorded image series, tracking software is available. We utilized arguably the fastest software, based on GPU processing, and made a few extensions to this software to decrease the time of processing microscopy recordings. Existing analysis methods to characterize the motion of proteins in live cells from the trajectories obtained after tracking are described.

Translating protein trajectories to biological implications, such as protein interactions, requires the classification of protein motion within the trajectories. Visualization of the spatial organization of protein motion can reveal where the protein interacts with cellular structures, since binding of proteins to such structures often reduces their diffusion speed. In chapter 3 we describe an analytical framework to determine in which diffusion state a molecule is residing during the course of its trajectory. We compared different quantification measures for utilization within this framework to classify between trajectory segments to two diffusion states (populations of different diffusion coefficients) using realistic simulated datasets. Diffusion classification can be applied to determine state lifetimes, and super-resolution like images of the diffusion states. A gyration based diffusion classification and a Bayesian approach were the most accurate in diffusion classification, where the gyration method required roughly thousand fold less 
computational time. For realistic experimental parameters and a diffusive system with an order of magnitude difference between the diffusion coefficients of the two populations, a classification correctness around $85 \%$ was achieved. This was enough to visually detect distinct zones of slowed diffusion in simulations and experimental data.

The SNAP-tag is an intrinsically monovalent and highly specific genetic tag for attaching a fluorescent organic dye to a protein of interest. A careful choice of the fluorescent substrate for SNAP-tag is important, as many labelled substrates either suffer from blinking and rapid photobleaching or high nonspecific staining. Since little information was available on the optimal choice of fluorescent labels for use in SNAP-tag at the single molecule level, in chapter 4 we evaluated 6 green- and 16 red-excitable dyes for their suitability. We determined the nonspecific binding levels in three different cell lines and the photostability of these dye conjugates, which are arguably the most important parameters to optimize in single-molecule tracking experiments, but appear to be unpredictable beforehand. We found using SNAP-tag fused EGFR that Dy 549 and CF 640 are the best dyes amongst the ones tested. These dyes only showed approximately 10 nonspecifically attached dyes (i.e. $<5 \%)$ per field of view $\left( \pm 2,000 \mu \mathrm{m}^{2}\right)$ in all cell lines tested. When specifically attached, these dyes had an expected photostability of 53,000 and 57,000 photons (Dy 549 and CF640 respectively), which is roughly an order of magnitude more than for autofluorescent proteins (e.g. 8,000 photons for eYFP ${ }^{28}$ ). With a mix of Dy 549 and CF640 substrates we could systematically obtain a dual-colour labelling of EGFR.

Whereas there is a strong link of dysregulation of ErbB proteins to carcinogenesis, the response in current anti-cancer therapy based on ErbB antagonists is limited. This motivates a better molecular understanding of such antagonists to secure a more stable signalling inhibition. In chapter 5 we recorded trajectories of EGF receptor, and determined the characteristics of the two population diffusion system. We noticed that the diffusion speed of the fast fraction relates to the clustering state of the receptor by validating the relation using known dimerization conditions, i.e. free resting, liganded and pre-clustered receptors. In a cellular environment with receptor antagonists, we found additional indications for the following model of EGFR dimerization in antagonistic conditions. Whereas cetuximab inhibited receptors can form relatively stable dimers due to an alternative extracellular confirmation distinct from the back-to-back ligand-induced formation, kinase inhibited receptors do not form stable dimers. The extracellular dimerization arms form only a weak connection, and stable dimers cannot be formed because the kinase domains cannot bind each other. Upon cetuximab binding to EGFR, ErbB3 shows increased diffusion in a cell line having a beneficial response on dual-target inhibition of EGFR and ErbB3, and shows decreased diffusion again upon subsequent addition of EGF. This indicates that ErbB3 is entangled with the signalling role of EGFR, and provides preliminary details why combination therapies, such as cetuximab treatment in combination with an anti-ErbB3 treatment, can be beneficial in some cell lines.

In chapter 6 we demonstrated that single receptors on the plasma membrane can be tracked whilst locally actuating a subpopulation of receptors using a functionalized AFM tips. This permits a local presentation of receptor stimuli with the possibility to monitor the response of the receptor proteins individually. First the bottom part of AFM tips were milled to obtain a tip-cell contact area of approximately $2 \times 2 \mu \mathrm{m}$, such that several ligand molecules are presented to the receptors of the cell. Next cysteine mutated EGF was attached to an amino-coated milled AFM tip via a flexible heterobifunctional polyethylene glycol (PEG) crosslinker with reactive NHS and maleimid group $^{29}$. An immunofluorescence assay was devised to confirm that cysteine mutated EGF attaches specifically to the reactive maleimid group on the PEG linker. Upon inducing the ligandreceptor (EGF - EGFR) interaction in live cells, we observed a stop of diffusion of the preferred hetero-dimerization partner (ErbB2) of the actuated receptor. This immobilization was only observed around the tip-cell contact site whereas receptors at a different location in the same cell were still mobile. 



\section{Samenvatting (in Dutch)}


Vrije vertaling van de origineel Engelstalige samenvatting. Deze vertaling biedt geen garanties op een juiste weergave van de Engelstalige samenvatting. Voor wetenschappelijk gebruik en in geval van tegenstrijdigheid tussen de vertaling en de oorspronkelijk Engelstalige samenvatting ("Summary"), is de originele Engelstalige versie leidend.

Dit proefschrift beschrijft nieuwe methodieken en toepassingen voor single-molecule tracking, een microscopische techniek om enkele individuele eiwit moleculen in levende cellen te volgen. De methodieken zijn toegepast om het signaleringsmechanisme van de ErbB receptorfamilie te bestuderen, in het bijzonder de epidermal growth factor receptor (EGFR). De ErbB receptorfamilie valt onder de receptor tyrosine kinases (RTK), en is betrokken in de vorming en progressie van tumoren. De ontwikkeling van nieuwe medicijnen die aangrijpen op deze receptoren is daarom van belang. Deze receptoren zijn over het plasmamembraan van de cel georganiseerd in dynamische samenstellingen (clustering), en ondergaan belemmerde diffusie op nano- en microniveau. Er wordt gedacht dat dit dynamische gedrag van receptoren een cruciale mechanistische rol speelt in de versterking en gevoeligheid van signaaltransductie binnen de cel. We hebben laten zien dat de diffusiesnelheid van EGFR gerelateerd is aan zijn dimerisatietoestand, een essentiële stap binnen de rol van de receptor om signalen van buiten te cellen te laten resulteren in bepaalde intercellulaire processen, zoals apoptose of celdeling. Kwaadaardige tumoren kunnen ontstaan door ontregeling van de receptorsignalering veroorzaakt door ontregelde dimerisatietoestanden. Door medicijnen geïnduceerde veranderingen in dimerisatietoestanden en de receptor organisatie (clustering) zijn daarom belangrijke onderwerpen in therapeutisch kankeronderzoek.

De gemeten single-molecule tracking data van EGFR en ErbB3 hebben veranderingen in de diffusie van deze receptoren in tumorcellen onthuld, die wijzen op specifieke moleculaire interacties, onder invloed van medicijnen die specifiek ontworpen zijn om zulke moleculaire interacties te voorkomen. Voordat we in staat waren om trajecten van EGFR te meten, en te analyseren op dimerisatie en interacties met andere cellulaire structuren, hebben we een microscoop voor dat doel gebouwd, een framework bedacht om trajecten te analyseren op verschillende diffusiepopulaties (populaties met verschillende diffusiecoëfficiënten), en een bestaand protein-tag fluorescerend label systeem - SNAP-tag - doorontwikkeld tot het niveau van individuele moleculen (single-molecule). Het in dit proefschrift beschreven onderzoek biedt de mogelijkheid om dit label systeem te gebruiken voor toepassingen in het afbeelden en volgen van individuele moleculen (single-molecule tracking), en om het framework te gebruiken voor classificatie van meerdere diffusiepopulaties binnen gemeten trajecten afgelegd door eiwitten zodat de dynamische eiwitbewegingen en interacties in levende cellen bestudeerd kunnen worden. Het proefschrift eindigt met een conceptmeting waarbij we laten zien dat lokaal geïnduceerde receptor activatie door ligand gefunctioneerde atomaire krachtenmicroscoop $(A F M)$ tips aanvullende inzichten in de moleculaire interacties kan brengen wanneer dit gecombineerd wordt met het volgen van individuele eiwit moleculen.

Hoofdstuk 1 bevat een introductie tot de ErbB receptorfamilie en hun moleculaire interacties; het concept van de single-molecule tracking techniek en de huidige beschikbare fluorescentie labels; en tot gecontroleerde receptor activatie middelen met een nadruk op strategieën om AFM tips te functionaliseren (te voorzien van eiwitten). In hoofdstuk 2 beschrijven we de voorwaarden en gerealiseerde implementatie voor een fluorescentie microscoop met single-molecule gevoeligheid waarmee individuele eiwitten met een fluorescerend label gevolgd kunnen worden in levende cellen. We hebben laten zien dat solid-state lasers voor deze toepassing een kostentechnisch gunstig alternatief zijn voor de meer gebruikte gas lasers, zonder dat de kwaliteit van de afbeeldingen er onder lijdt. Een ronddraaiende diffusor in een Köhler belichtingsschema is gebruikt om een incoherente lichtbron met een hoge intensiteit te krijgen zonder gebruik te maken van een meer gebruikt schema met optische fibers. De microscoop kan individuele fluorescerende moleculen (organic dyes) detecteren met een lokalisatie nauwkeurigheid van 20 
nanometer binnen 10 milliseconden, wat voldoet aan de voorwaarden om EGFR te kunnen volgen. Om de afgelegde trajecten te reconstrueren uit een serie van microscoopopnames is bestaande software beschikbaar. We hebben software gebruikt die gebruik maakt van de GPU (grafische kaart), en hebben enkele uitbreidingen op deze software gemaakt om de tijdsduur om microscoopopnames te verwerken te verminderen. Verder worden in dit hoofdstuk bestaande methoden beschreven om de beweging van eiwitten uit hun afgelegde trajecten te analyseren.

Voor het afleiden van biologische implicaties, zoals interacties tussen eiwitten, uit afgelegde trajecten van eiwitten is een classificatie binnen zulke trajecten van de eiwitbewegingen nodig. Het visualiseren van de ruimtelijke organisatie van deze eiwitbewegingen kan onthullen waar een eiwit interacties heeft met cellulaire structuren, omdat zulke interacties doorgaans resulteren in verminderde diffusiesnelheid van het eiwit. In hoofdstuk 3 beschrijven we een analytisch framework waarmee kan worden bepaald welke diffusietoestanden een molecuul aanneemt op elke positie van het afgelegde traject. We hebben verschillen kwantificatiemethoden vergeleken voor gebruik binnen dit framework om trajectsegmenten te classificeren in twee diffusiepopulaties met behulp van realistisch gesimuleerde datasets. Classificatie van de diffusie kan worden toegepast om de levensduur van de diffusietoestand (diffusie behorende bij een van de populaties) te bepalen, en super-resolutie achtige afbeeldingen op te stellen van de diffusietoestanden. Een methode die gebruikt maakt van de gyration-straal of een Bayesiaanse methode bleken de hoogste accuraatheid voor diffusieclassificatie te hebben, waarbij de op gyration gebaseerde methode ongeveer duizend maal minder rekentijd nodig heeft. Een classificatie correctheid van ongeveer $85 \%$ is gehaald voor experimenteel realistische parameters en een diffusie systeem met een orde van grootte verschil tussen de diffusie coëfficiënten van beide populaties. Dit was genoeg om structuren waar te nemen in de zones met afgenomen diffusie zowel in simulaties als in experimentele data (in vivo).

De SNAP-tag is een intrinsiek monovalente genetische protein-tag waaraan zeer specifiek een fluorescerend substraat (met organic dye) kan worden gebonden; de tag zelf kan aan een eiwit naar keuze gekloneerd worden. Een afgewogen keuze van het fluorescerende substraat voor de SNAPtag is belangrijk, omdat veel fluorescerende substraten slecht presteren wat betreft blinking en snelle bleking (photobleaching) of hoge niet-specifieke kleuring. Omdat er weinig informatie beschikbaar was wat betreft een optimale keuze van fluorescerende substraten voor de SNAP-tag op het niveau van individuele moleculen, hebben we in hoofdstuk 4 gekeken naar de geschiktheid van 6 groen- en 16 rood-geëxciteerde fluorescentie labels. We hebben de mate van niet-specifieke kleuring door deze labels bepaald in drie verschillende cellijnen, alsmede de bleking karakteristieken. Deze eigenschappen worden gezien als de belangrijkste parameters om te optimaliseren in single-molecule tracking experimenten, en bleken niet van te voren te voorspellen. Onze conclusies zijn dat van de geteste substraten die met Dy 549 en CF 640 de beste zijn in ons systeem, een met een SNAP-tag gefuseerd EGFR. Deze substraten vertoonden ongeveer 10 nietspecifieke bindingen ( $<5 \%$ t.o.v. specifiek gebonden substraten) per gezichtsveld (field of view) van ongeveer $2^{\prime} 000 \mu \mathrm{m}^{2}$ in alle geteste cellijnen. Voor de specifiek gebonden substraten geldt dat zij een gemiddelde levensduur (voor bleking) hebben van respectievelijk 53'000 en 57'000 fotonen voor Dy 549 en CF 640. Dit is ongeveer een orde van grootte meer dan voor auto-fluorescerende eiwitten (bijvoorbeeld 8'000 fotonen voor eYFP ${ }^{28}$ ). Met een mengsel van Dy 549 en CF 640 substraten konden we systematisch een twee-kleuren kleuring van SNAP-EGFR bewerkstelligen. Hoewel er een sterk verband is aangetoond tussen ontregelde ErbB receptoren en tumorgroei, is de werking van op deze receptoren gerichte antikankermedicijnen die moleculair ingrijpen nog beperkt. Een beter moleculair begrip over de werking van zulke medicijnen is nodig voor een betere onderdrukking van de receptorsignalering. In hoofdstuk 5 hebben we trajecten van EGFR geanalyseerd middels een dynamisch diffusiesysteem met twee populaties. We hebben een verband gelegd tussen de diffusiesnelheid van de snellere populatie en de dimerisatie of clustering toestand van de receptor. Dit verband hebben we geverifieerd met van tevoren bekende 
dimerisatie toestanden, namelijk met vrije (in rust), ligand gebonden, en pre-clustered receptoren. Gebruik makend van dit verband konden we aanwijzingen vinden voor het volgende moleculaire model wanneer receptor onderdrukkende moleculen in de omgeving van de cel aanwezig waren: hoewel door cetuximab onderdrukte receptoren relatief stabiele dimeren kunnen vormen dankzij een alternatieve extracellulaire conformatieverandering, die verschillend is van de door een ligand geïnduceerde back-to-back formatie, kunnen door kinaseremmers onderdrukte receptoren geen stabiele dimeren vormen. De extracellulaire dimerisatiearmen vormen dan slechts een zwakke verbinding, en stabiele dimeren worden niet gevormd omdat de kinase domeinen onderdrukt zijn en geen binding meer met elkaar kunnen maken. Wanneer cetuximab aan EGFR bindt, versnelt de diffusie van ErbB3 receptoren in een cellijn die gevoelig is voor een combinatietherapie van EGFR en ErbB3; deze diffusie vertraagt weer nadat EGF ligand is toegevoegd aan het celmedium. Dit vormt een aanwijzing dat ErbB3 betrokken is in het signaleringsmechanisme van EGFR, wat weer een aanwijzing kan zijn waarom sommige cellijnen gevoelig zijn voor combinatietherapieën, zoals een behandeling met cetuximab en antilichaam voor ErbB3.

Tot slot hebben we in hoofdstuk 6 laten zien dat individuele receptoren op het plasmamembraan gevolgd kunnen worden terwijl we een subpopulatie van receptoren activeren met behulp van ligand gefunctioneerde AFM tips. Dit stelt ons in staat om lokaal receptoren te stimuleren terwijl tegelijkertijd de respons van individuele receptoren kan worden bekeken. Hiervoor is allereerst de onderkant van AFM tips verwijderd met een focussed ion beam zodat het tip-cel contactoppervlak ongeveer $2 \times 2 \mu \mathrm{m}$ wordt, waardoor meerdere ligand moleculen zich aan receptoren op de cel kunnen presenteren. Vervolgens zijn cysteïne-gemuteerde EGF moleculen op een amino-gecoate AFM tip gezet middels een flexibele en hetero-functionele polyethylene glycol (PEG)-verbinder met reactieve NHS en maleimide groepen ${ }^{29}$. Een immunofluorescentie assay is opgesteld om te bevestigen dat de cysteïne-gemuteerde EGF moleculen specifiek aan de reactieve maleimide groep van de PEG-verbinder bindt. Nadat de ligand-receptor (EGF-EGFR) interactie was geïnduceerd in levende cellen, zagen we dat ErbB2 receptoren, een geprefereerde hetero-dimerisatie partner van EGFR, ophielden met bewegen. Deze bewegingsstilstand was specifiek op de plek van het tip-cel contact, en receptoren op een andere plek in dezelfde cel vertoonden nog steeds diffusie.

Samenvattend kan worden gesteld dat er nog genoeg ruimte voor verbetering is om eiwitten te voorzien van fluorescerende labels op de schaal van individuele moleculen (single-molecule level), en om methoden uit te breiden die erop gericht zijn om de door eiwitten afgelegde trajecten te analyseren, dat biofysici worden aangemoedigd de single-molecule tracking techniek nog verder te verbeteren zodat haar volledige potentie kan worden gebruikt. 


\section{References}

1. Kusumi, A. et al. Paradigm shift of the plasma membrane concept from the two-dimensional continuum fluid to the partitioned fluid: high-speed single-molecule tracking of membrane molecules. Annu. Rev. Biophys. Biomol. Struct. 34, 351-78 (2005).

2. Lew, M. D., Thompson, M. a, Badieirostami, M. \& Moerner, W. E. In vivo Three-Dimensional Superresolution Fluorescence Tracking using a Double-Helix Point Spread Function. Proc. Soc. Photo. Opt. Instrum. Eng. 7571, $75710 Z$ (2010)

3. Thompson, M. a, Lew, M. D., Badieirostami, M. \& Moerner, W. E. Localizing and tracking single nanoscale emitters in three dimensions with high spatiotemporal resolution using a double-helix point spread function. Nano Lett. 10, 211-8 (2010).

4. Badieirostami, M., Lew, M. D., Thompson, M. a \& Moerner, W. E. Three-dimensional localization precision of the double-helix point spread function versus astigmatism and biplane. Appl. Phys. Lett. 97, 161103 (2010)

5. Low-Nam, S. T. et al. ErbB1 dimerization is promoted by domain co-confinement and stabilized by ligand binding. Nat. Struct. Mol. Biol. 18, 1244-1249 (2011).

6. Cutler, P. J. et al. Multi-color quantum dot tracking using a high-speed hyperspectral line-scanning microscope. PLoS One 8, e64320 (2013).

7. Kholodenko, B. N., Hancock, J. F. \& Kolch, W. Signalling ballet in space and time. Nat. Rev. Mol. Cell Biol. 11, 414-426 (2010).

8. Benke, A., Olivier, N., Gunzenhäuser, J. \& Manley, S. Multicolor single molecule tracking of stochastically active synthetic dyes. Nano Lett. 12, 2619-24 (2012).

9. Wieser, S., Axmann, M. \& Schütz, G. J. Versatile analysis of single-molecule tracking data by comprehensive testing against Monte Carlo simulations. Biophys. J. 95, 5988-6001 (2008).

10. Howarth, M. et al. Monovalent, reduced-size quantum dots for imaging receptors on living cells. Nat. Methods 5, 397-9 (2008).

11. Liu, H. Y. \& Gao, X. Engineering monovalent quantum dot-antibody bioconjugates with a hybrid gel system. Bioconjug. Chem. 22, 510-7 (2011).

12. Farlow, J. et al. Formation of targeted monovalent quantum dots by steric exclusion. Nat. Methods 10, 1203-5 (2013).

13. Pinaud, F. F. et al. Quantum dots for live cells, in vivo imaging, and diagnostics. Science 307, 538-44 (2005).

14. Pinaud, F., Clarke, S., Sittner, A. \& Dahan, M Probing cellular events, one quantum dot at a time. Nat. Methods 7, 275-85 (2010).
15. Moriai, T., Kobrin, M. S., Hope, C., Speck, L. \& Korc M. A variant epidermal growth factor receptor exhibits altered type alpha transforming growth factor binding and transmembrane signaling. Proc. Natl. Acad. Sci. U. S. A. 91, 10217-21 (1994)

16. Vlasov, I. I. et al. Molecular-sized fluorescent nanodiamonds. Nat. Nanotechnol. 9, 54-8 (2014).

17. Laschat, S. et al. Discotic liquid crystals: from tailormade synthesis to plastic electronics. Angew. Chem. Int. Ed. Engl. 46, 4832-87 (2007).

18. Sergé, A., Bertaux, N., Rigneault, H. \& Marguet, D. Dynamic multiple-target tracing to probe spatiotemporal cartography of cell membranes. Nat. Methods 5, 687-94 (2008).

19. Dietrich, C., Yang, B., Fujiwara, T., Kusumi, A. \& Jacobson, K. Relationship of lipid rafts to transient confinement zones detected by single particle tracking. Biophys. J. 82, 274-84 (2002).

20. Simson, R., Sheets, E. D. \& Jacobson, K. Detection of temporary lateral confinement of membrane proteins using single-particle tracking analysis. Biophys. J. 69, 989-93 (1995)

21. Goulian, M. \& Simon, S. M. Tracking single proteins within cells. Biophys. J. 79, 2188-98 (2000).

22. Ledford, H. Immunotherapy's cancer remit widens. Nature 497, 544 (2013).

23. Sako, Y., Minoghchi, S. \& Yanagida, T. Singlemolecule imaging of EGFR signalling on the surface of living cells. Nat. Cell Biol. 2, 168-72 (2000).

24. Xiao, Z. et al. Single-molecule study of lateral mobility of epidermal growth factor receptor 2/HER2 on activation. J. Phys. Chem. B 112, 4140-5 (2008).

25. Chung, I. et al. Spatial control of EGF receptor activation by reversible dimerization on living cells. Nature 464, 783-7 (2010).

26. Orr, G. et al. Cholesterol dictates the freedom of EGF receptors and HER2 in the plane of the membrane. Biophys. J. 89, 1362-73 (2005)

27. Rong, G. \& Reinhard, B. M. Monitoring the size and lateral dynamics of ErbB1 enriched membrane domains through live cell plasmon coupling microscopy. PLoS One 7, e34175 (2012)

28. Harms, G. S., Cognet, L., Lommerse, P. H., Blab, G. A. \& Schmidt, T. Autofluorescent proteins in singlemolecule research: applications to live cell imaging microscopy. Biophys. J. 80, 2396-408 (2001)

29. Ebner, A. et al. Functionalization of probe tips and supports for single-molecule recognition force microscopy. Top. Curr. Chem. 285, 29-76 (2008) 



\section{Dankwoord}

En dan is het af en klaar, ook al is het dat nooit. Ik heb gezegd. Vier en een half jaar van uitproberen, testen, frustraties, doorzetten, doorbraken en jezelf ontdekken zitten er op. Een periode waarin ik bijzonder veel heb geleerd, grotendeels dankzij degenen met wie ik heb samengewerkt. Rest mij daarom de belangrijke taak om hen te bedanken die mij geholpen en ondersteund hebben.

Allereerst mijn promotor en co-promotor. Vinod, je zorgde altijd dat ik in de goede richting keek, en bleef rondkijken voor alle mogelijkheden en samenwerkingen. Het is tevens jouw gave om mij weer vol zelfvertrouwen en enthousiasme te krijgen, ook tijdens perioden van frustraties. Hans, bedankt voor de talrijke discussies die wij altijd voerden, ik heb deze altijd zeer kunnen waarderen. Van de koffietafel tot Linz en Barcelona, jij bleef altijd zeer scherp en kritisch kijken naar ideeën en mogelijkheden. Mijn pragmatische houding in het onderzoek was jou soms iets te vluchtig; jouw interesse in de details ging mij soms te ver, maar wakkerde mij op bepaalde momenten wel aan om het nog beter uit te zoeken, wat dan vaak tot nieuwe inzichten leidde. Bedankt voor je kritische en behulpzame begeleiding. Vinod en Hans, jullie gaven mij direct aan het begin van mijn project al heel veel vrijheid. Hierdoor heb ik wel het eerste jaar erg vanuit het diepe moeten zoeken naar mijn weg in de wetenschap, en ondersteuning in het chemisch en biologisch lab. Mijn zelfstandigheid is op de proef gesteld, maar is nog sterker geworden.

Many thanks as well to the members of the the NanoActuate project consortium. You played an important role in the experimental parts of my project, but also in understanding the importance of the fundamental molecular cell biology. I would especially like to acknowledge Micha for making crucial biochemical compounds, and Jenny for sharing all plasmid constructs which allowed the tracking of the HER proteins and for the good collaboration concerning the practicalities of single molecule tracking using SNAP-tag.

I enjoyed several other collaboration established during my project. The first collaboration started right in the first months with a visit to the lab of Peter Hinterdorfer of the University of Linz in Austria to learn the art of protein coupling from his group. I was openly welcomed by Andreas Ebner, who appeared to be a very enthusiastic person and was always willing to answer my many questions; thank you for all your help.

When I was struggling to find a good set of multi-colour SNAP substrate fluorophores, Ivan Corrêa from New England Biolabs kindly responded by freely providing many new substrates for testing. Later this resulted in a manuscript (Chapter 4), for which you also provided very helpful feedback to improve the quality of my writing.

For the tracking of the proteins in the video recordings I am grateful to Keith Lidke for kindly providing his software, and to Peter Relich for explaining the software and being open to my comments. Thanks to your quick responses by e-mail and Skype the time difference was no issue. 
Toen alle methodes begonnen te werken, zocht ik naar een echte toepassing van de techniek. Een erg boeiende en leerzame samenwerking was daarom die met de medische oncologie groep van Liesbeth de Vries aan de Rijksuniversiteit Groningen. Deze enthousiaste mensen lieten mij razendsnel de klinische relevantie van mijn fundamentele onderzoek doen inzien in de vorm van hun onderzoek naar anti-kanker medicijnen tegen de receptoren familie die ik kon volgen (tracken) op de microscoop. Het bleek dat ondanks veel onderzoek het moleculaire begrip van dit soort medicijnen nog beperkt is. Dankzij jullie kennis van de receptoren en hun reactie op medicijn behandeling heb ik hopelijk een interessante extra blik op het moleculaire gedrag van de receptoren kunnen geven. Anton, Thijs, Arjan, Steven, en Liesbeth, heel erg bedankt voor jullie hulp en enthousiasme.

Tot slot wil ik Sandra en Alessandra van de afdeling Tumor Immunology van de Radboud Instiute for Molecular Life Sciences hartelijk danken voor hun interesse en toepassing in de state classificatie tools die ik heb ontwikkeld (Chapter 3). Fijn dat jullie de moed niet opgaven om de tools ook op jullie data werkend te krijgen, ondanks de vele aanpassingen die ik moest maken in de scripts. Dankzij jullie kreeg ik echt het besef van de biologisch relevantie van dergelijke tools. Er ligt een mooi manuscript klaar, en het was erg leerzaam om hieraan een bijdrage te leveren.

Natuurlijk wil ik ook al mijn collega's van de vakgroep bedanken, zeker diegenen die mij hebben geïntroduceerd en ondersteund met de vele labtechnieken en meer. Speciaal wil ik noemen: Tom voor het leren kweken en transfecteren van cellen inclusief de vele interessant en nuttige details; Yvonne voor uitgebreide ondersteuning van de celkweek; Kirsten voor alle kennis en hulp over (bio)chemische technieken; Irene voor hulp bij allerlei biochemische tests en het knippen en plakken in DNA; Robert en Kees voor het meedenken en maken van een perfecte AFM voor eiwit presentatie aan cellen; Sjashank, Marieke, Cicerone, Burçu, Rolf, Niels, Martijn, en Kim voor alle hulp en discussies; Sylvia voor de administratieve ondersteuning. Naast de werk gerelateerde aspecten, wil ik ook graag iedereen van de vakgroep bedanken voor het zijn van leuke en enthousiaste collega's. Tot slot wil ik mijn kantoorgenoten bedanken voor alle gesprekjes van dag tot dag, Martijn, Liesbeth, Anja, Kristian, en Carla, jullie waren erg fijne kantoorgenoten.

Het spijt me heel erg als ik nu iemand vergeten ben bij naam te noemen, maar mijn dank is niet minder groot!

Naast mijn collega's wil ik zeker ook mijn vrienden bedanken voor het beleven van allerlei leuke momenten. Met velen deel ik de gezamenlijke hobby en levensstijl van sportklimmen. Dankzij jullie kon ik lekker bijkomen van de doordeweekse inspanningen gedurende de vele weekenden en vakanties bij de rotsen, maar natuurlijk ook onder het genot van een biertje bij jullie of mij thuis.

Mijn familie, en in het bijzonder mijn ouders Hans en Ineke, en broer en zus, Michiel en Noor, bedankt voor al jullie steun, interesse en begrip tijdens mijn promotietraject.

Lieve Iris, bedankt voor alles wat je voor mij betekent. Ook dankzij jouw liefde, steun en vertrouwen is dit proefschrift er gekomen.

Peter 


\section{List of publications}

\section{Papers}

- P.J. Bosch, J.S. Kanger and V. Subramaniam, Classification of dynamical diffusion states in single molecule tracking microscopy, Biophysical Journal, 2014, in press.

- S. de Keijzer, M.B.M. Meddens, S. Schwartz, P.J. Bosch, B. Joosten, C.G. Figdor, J.S. Kanger, V. Subramaniam, D.S. Lidke and A. Cambi, Cortical microtubules shape GPCR spatiotemporal membrane organization and signalling, submitted.

- P.J. Bosch, I.R. Corrêa Jr., M.H. Sonntag, L. Brunsveld, J.S. Kanger and V. Subramaniam, A quantitative survey of dyes for labeling SNAP tag fused membrane proteins for multicolor single molecule tracking microscopy in live cells, Biophysical Journal, 2014, in press.

\section{Conference talks}

- P.J. Bosch, J.S. Kanger and V. Subramaniam, State classification of two-population dynamic diffusion systems: comparison of various analysis methods, Quantitative Bioimaging Conference in Albuquerque, New Mexico, USA, 11-12 January, 2013. 


\section{Curriculum Vitae}

Peter Bosch was born on the 13th of July 1984 in Leeuwarden. After finishing the Stedelijk Gymnasium in Leeuwarden, he started his study in applied physics at the University of Twente in 2002. During the study, of which 8 months were spent abroad for internships and a study tour, he had a wide interest in quantum physics and optics, while keeping a close eye on the more applied field of biophysics. In 2007, he went on an internship in biophysics at the National Institute for Nanotechnology (NINT) of the National Research Council (NRC) of Canada with Prof. Dr. Nils Petersen and worked on combining information obtained by AFM and TOF-SIMS on cell sections. Thereafter he chose to do his graduation project in condensed matter physics about correlated electron transport within the group of Prof. Dr. ir. Hans Hilgenkamp. He graduated a year later for the university degree. In the summer of 2009 he chose to start a $\mathrm{PhD}$ project that combined biophysics with molecular cell biology in the nanobiophysics group of Prof. Dr. Vinod Subramaniam at the University of Twente. The project focused on the development of tools to utilize in single-molecule tracking microscopy to study the signalling mechanism of plasma membrane resident receptors. The most important results are presented in this dissertation, together with applications to receptors of the ErbB family, and in particular the epidermal growth factor receptor (EGFR). 